\title{
THE SEARCH FOR HIGHER TWIST EFFECTS IN THE NEUTRON SPIN STRUCTURE FUNCTION $g_{2}^{n}\left(x, Q^{2}\right)$
}

\author{
A Dissertation \\ Presented to \\ The Faculty of the Department of Physics \\ The College of William and Mary in Virginia \\ In Partial Fulfillment \\ Of the Requirements for the Degree of \\ Doctor of Philosophy
}

by

Kevin M. Kramer

2003 


\section{APPROVAL SHEET}

This dissertation is submitted in partial fulfillment of

the requirements for the degree of

Doctor of Philosophy

Kevin M. Kramer

Approved, August 2003

Todd Averett

David S. Armstrong

J. Michael Finn

J. Dirk Walecka

Jian-Ping Chen

Jefferson Lab 
To the memory of John M. Kramer (1918-2003) 


\section{CONTENTS}

ACKNOWLEDGMENTS ................. xi

LIST OF TABLES $\ldots \ldots \ldots \ldots \ldots \ldots \ldots \ldots$ xv

LIST OF ILLUSTRATIONS . . . . . . . . . . . xxiii

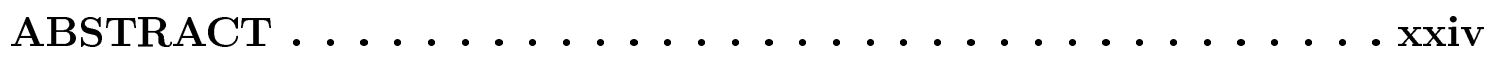

CHAPTER

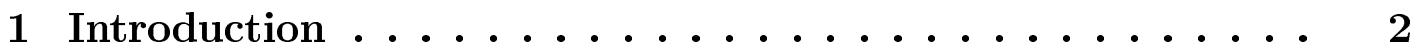

2 Inclusive Electron Scattering $\ldots \ldots \ldots \ldots \ldots$

2.1 Introduction . . . . . . . . . . . . . . . . . 4

2.2 The Framework of Polarized Inclusive Electron Scattering . . . . 5

2.2.1 Kinematic Variables. . . . . . . . . . . . . . . . 5

2.2.2 Deriving the differential cross-section $\ldots \ldots \ldots \ldots$

2.2.3 The leptonic and hadronic tensors . . . . . . . . . . 7

2.2.4 The structure functions . . . . . . . . . . . . . 9

2.3 Types of inclusive electron scattering $\ldots \ldots \ldots \ldots$

2.3.1 General description of electron scattering on a nucleus . . . 10

2.3.2 Polarized elastic scattering on the nucleon . . . . . . . . 11

2.3.3 Polarized elastic scattering on ${ }^{3} \mathrm{He} \ldots \ldots \ldots$. . . . . . 14

2.3.4 The effective polarization of the neutron in the ${ }^{3}$ He nucleus 15

2.3.5 Polarized quasi-elastic scattering on ${ }^{3} \mathrm{He} \ldots \ldots \ldots$

2.3.6 Scattering in the resonance and deep inelastic region . . . 18 


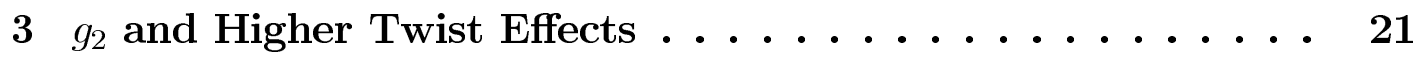

3.1 Why measure $g_{2} ? \ldots \ldots \ldots \ldots \ldots \ldots \ldots$

3.2 Deriving the Wandzura-Wilczek relation . . . . . . . . . 22

3.2.1 A description of the operator product expansion . . . . . . 22

3.2.2 Twist two operators in the operator product expansion . . 23

3.2.3 QCD coefficients in the operator product expansion . . . . 25

3.2.4 Extracting relations for $g_{1}$ and $g_{2} \ldots \ldots \ldots \ldots \ldots$

3.2.5 Twist three operators . . . . . . . . . . . . . . . 29

3.3 Models of $g_{2} \ldots \ldots \ldots \ldots \ldots \ldots \ldots \ldots$

3.3.1 The parton model . . . . . . . . . . . . . . . 31

3.3.2 Bag Models of $g_{2} \ldots \ldots \ldots \ldots \ldots$

3.4 Experimental data on $g_{2} \ldots \ldots \ldots \ldots \ldots \ldots$

4 Experimental Overview $\ldots \ldots \ldots \ldots \ldots$

4.1 Goals of E97-103 . . . . . . . . . . . . . . . . . 40

4.1 .1 Physics goals . . . . . . . . . . . . . . . 40

4.1 .2 Asymmetry method . . . . . . . . . . . . . . . . . 41

4.2 Experimental Setup . . . . . . . . . . . . . . . . . . 43

5 The Electron Beam and Beam line Apparatus . . . . . . 46

5.1 Jefferson Lab and CEBAF . . . . . . . . . . . . . 46

5.1 .1 The accelerator facility . . . . . . . . . . . 46

5.1 .2 The polarized source $\ldots \ldots \ldots \ldots$. . . . . . 47

5.2 Measuring the beam energy $\ldots \ldots \ldots \ldots$. . . . . . 49

5.2.1 Arc energy measurements . . . . . . . . . . . . 49

5.2 .2 eP energy measurements . . . . . . . . . . . . . 51

5.2 .3 Beam energy used for E97-103 . . . . . . . . . . . . 52

5.3 Measuring the beam polarization $\ldots \ldots \ldots \ldots \ldots$ 
5.3 .1 Møller polarimeter . . . . . . . . . . . . . . . 52

5.3 .2 Compton polarimeter . . . . . . . . . . . . . . 54

5.3.3 Beam polarization for E97-103 _ . . . . . . . . . 57

5.4 Beam helicity, charge and position . . . . . . . . . . . 58

5.4 .1 Beam helicity . . . . . . . . . . . . . 58

5.4 .2 Measuring Beam Charge . . . . . . . . . . . . . 59

5.4.3 Beam charge asymmetry feedback system . . . . . . . . . . 62

5.4 .4 Beam position monitors . . . . . . . . . . . . 64

5.4 .5 Raster . . . . . . . . . . . . . . . 66

6 The Polarized ${ }^{3}$ He Target System _. . . . . . . . . 68

6.1 Why a Polarized ${ }^{3}$ He Target? . . . . . . . . . . . . 68

6.2 A Quick Word About Other ${ }^{3}$ He Polarization Techniques . . . . . 69

6.3 Jefferson Lab Polarized ${ }^{3}$ He Target Overview . . . . . . . . . . . . 69

6.4 Polarizing ${ }^{3}$ He Using Rubidium $\ldots \ldots \ldots \ldots \ldots \ldots$

6.4.1 Polarizing Rubidium . . . . . . . . . . . . . . 71

6.4.2 Polarizing ${ }^{3}$ He With Polarized Rubidium . . . . . . . . . 74

6.4.3 Hyperfine Splitting from the Nucleus $\ldots \ldots$. . . . . 78

6.5 Polarized ${ }^{3} \mathrm{He}$ Cell Construction . . . . . . . . . . . . . . . . 79

6.5.1 The Fabrication of the Glass Target Cells . . . . . . . . 79

6.5.2 The Cell Filling System . . . . . . . . . . . . . . . . . 82

6.5.3 Preparing for Cell Filling . . . . . . . . . . . . . 83

6.5.4 Measurement of the Cell Assembly Volume . . . . . . . . 86

6.5.5 Filling the Cell with Nitrogen and ${ }^{3} \mathrm{He} \ldots$. . . . . 88

6.6 Characterizing Target Cells . . . . . . . . . . . . . . . . . 90

6.6.1 External Dimensions of Cells used during E97-103 _ . . . . 90

6.6.2 Measuring the Total Internal Cell Volume . . . . . . . . . 92 
6.6.3 The External Volumes of the Cell Chambers . . . . . . . . 93

6.6.4 The dimensions of the $25 \mathrm{~cm}$ Water Cell . . . . . . . . . 95

6.6.5 Target Chamber Wall Thicknesses . . . . . . . . . . . . 95

6.6.6 Target Chamber End Window Measurement . . . . . . . . 97

6.6.7 Estimating the Interior Volumes of Target Cells . . . . . 98

6.6.8 Measuring the Cell Density . . . . . . . . . . . . . . . 99

6.7 Lasers and Optics . . . . . . . . . . . . . . . . . . . 100

6.7 .1 Polarizing Optics . . . . . . . . . . . . . . 100

6.7 .2 Optics Configuration . . . . . . . . . . . . . . . 101

6.8 The Magnetic Fields . . . . . . . . . . . . . . . . . . . 103

6.8.1 The Helmholtz Coils . . . . . . . . . . . . . . . . . 103

6.8.2 Mapping of Helmholtz Coils . . . . . . . . . . . . . 105

6.8.3 Field Direction of the Helmholtz Coils . . . . . . . 107

6.8.4 Calibration of RF coils . . . . . . . . . . . . 108

6.9 Measuring Target Polarization . . . . . . . . . . . . . . 110

6.9.1 The Adiabatic Condition . . . . . . . . . . . . . . . 110

6.9.2 The Bloch Equations . . . . . . . . . . . . . . . . 113

6.9.3 Adiabatic Fast Passage . . . . . . . . . . . . . . . . 115

6.9.4 NMR Polarimetry Setup . . . . . . . . . . . . . . 117

6.9.5 Extracting Polarization from the NMR Signal . . . . . 118

6.9.6 NMR Flux in the Pick-up Coils . . . . . . . . . . . . . 121

6.9.7 Measuring Cell Temperature and Density . . . . . . . . 126

6.9.8 The Gain of the Pick-Up Coils . . . . . . . . . . . . . . 129

6.9.9 Modifications to the NMR signal shape . . . . . . . . . 130

6.9.10 Polarization Loss due to AFP measurements . . . . . . . 134

6.9.11 Analysis of the NMR Signals from Water Cells . . . . . . 136

6.9 .12 EPR polarimetry . . . . . . . . . . . . . . . 140 
6.9.13 Combining Data from EPR and NMR . . . . . . . . . 145

6.10 Masing . . . . . . . . . . . . . . . . . . . 147

6.11 Reference Cell . . . . . . . . . . . . . . . . . . . . . 148

7 The Hall A Spectrometers and Detector Package . . . . . 150

7.1 Spectrometer Magnets . . . . . . . . . . . . . . . . 150

7.2 Detector Package . . . . . . . . . . . . . . . . . 152

7.2 .1 Overview . . . . . . . . . . . . . . . . . 152

7.2.2 Vertical Drift Chambers . . . . . . . . . . . . 153

7.2.3 Scintillator and Trigger Electronics . . . . . . . . . 156

7.2 .4 Gas C̆erenkov Detector . . . . . . . . . . . . . . . . 158

7.2.5 Lead-Glass Shower Detectors . . . . . . . . . . . . . . 159

7.2.6 Combined Particle Identification Efficiency . . . . . . . . 161

7.3 Data Acquisition System . . . . . . . . . . . . . . . . 162

8 Asymmetry Analysis . . . . . . . . . . . . 163

8.1 Extracting Raw Asymmetries . . . . . . . . . . . . 163

8.1 .1 Overview . . . . . . . . . . . . . . . . . 163

8.1.2 Creating N-tuples with ESPACE . . . . . . . . . . . 163

8.1.3 Extracting Charge and Dead-time Information . . . . . . 165

8.1.4 Analyzing N-tuples . . . . . . . . . . . . . . . . 168

8.2 Establishing Sign Convention _ . . . . . . . . . . . 169

8.3 Detector Cut Studies . . . . . . . . . . . . . . . . 170

8.3.1 Electron Identification Studies . . . . . . . . . . . 170

8.3.2 End Window Cut Studies . . . . . . . . . . . . 173

8.3.3 Acceptance Cut Studies . . . . . . . . . . . . . . . . 173

8.4 False Asymmetries . . . . . . . . . . . . . . . . 176

8.4.1 Removing Beam Ramping Periods . . . . . . . . . . . 176 
8.4.2 The Effect of Holding Field Misalignment . . . . . . . . . 177

8.4.3 Carbon Quasi-Elastic Analysis . . . . . . . . . . . . . . 178

8.4.4 End Window False Asymmetries . . . . . . . . . . . . 181

8.4.5 Calculating Error from False Asymmetry . . . . . . . . . 181

8.5 Nitrogen Dilution . . . . . . . . . . . . . . . . . . 182

8.5.1 Using the Reference Cell to Measure the Nitrogen Contribution . . . . . . . . . . . . . . . . 182

8.5.2 Dilution Factor from Fill Density . . . . . . . . . . 187

8.6 Elastic Asymmetry Check . . . . . . . . . . . . . . . . 187

8.7 Final Corrected Asymmetries _ . . . . . . . . . . . . . 189

9 Radiative Corrections . . . . . . . . . . . . . 191

9.1 Introduction to Radiative Corrections . . . . . . . . . . . . . 191

9.2 Methods of Calculating Radiative Corrections _ . . . . . . . . 192

9.2.1 Internal Radiative Corrections . . . . . . . . . . . . . . 192

9.2.2 External Radiative Corrections . . . . . . . . . . . . 193

9.2.3 The Radiative Corrections Procedure . . . . . . . . . . . 194

9.3 Elastic and Quasi-Elastic Radiative Corrections . . . . . . . . 195

9.3.1 Elastic Radiative Corrections _ . . . . . . . . . . . 195

9.3.2 Unpolarized Quasi-Elastic Cross-section . . . . . . . . . 196

9.3.3 Polarized Quasi-Elastic Radiative Corrections . . . . . . 197

9.3.4 Elastic and Quasi-elastic Cross-section Subtraction _. . . 200

9.4 Inelastic Radiative Corrections . . . . . . . . . . . . . . . . 202

9.4.1 Models for Radiative Corrections _ . . . . . . . . . . . 202

9.4.2 Summary of Inelastic Corrections _ . . . . . . . . . . 206

10 Results and Systematic Errors . . . . . . . . . . 212

10.1 Calculating $g_{1}^{3 \mathrm{He}}$ and $g_{2}^{3} \mathrm{He} \ldots \ldots \ldots \ldots 212$ 
10.2 Extracting Neutron Structure Functions from ${ }^{3} \mathrm{He}$. . . . . . . . . 212

10.3 Calculating $g_{2}^{\mathrm{WW}} \ldots \ldots \ldots \ldots$

10.4 Results for $g_{1}^{n}$ and $g_{2}^{n} \ldots \ldots$. . . . . . . . . . . 214

10.5 Summary of Systematic Errors . . . . . . . . . . . . . . 217

10.6 Conclusion . . . . . . . . . . . . . . . . 217

APPENDIX A

Magnetic Field Calibrations . . . . . . . . . . . . 220

APPENDIX B

Mapping of Holding Field Gradients . . . . . . . . . . . 222

APPENDIX C

Results of Field Direction Surveys . . . . . . . . . . . 224

BIBLIOGRAPHY . . . . . . . . . . . . . . 228

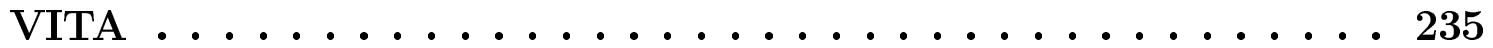




\section{ACKNOWLEDGMENTS}

I acknowledge that I need to write some acknowledgements. 


\section{LIST OF TABLES}

2.1 A table of kinematic factors $\ldots \ldots \ldots \ldots$

2.2 A table of more kinematic factors $\ldots \ldots \ldots \ldots$

3.1 The dimension (d), spin ( $n$ ) and twist ( $(t)$ of the operators for quarks $(\psi)$, gluons $\left(G_{\mu \nu}\right)$ and covariant derivatives $D^{\mu} \ldots \ldots \ldots \ldots$

3.2 Description of the DIS world data set on $g_{1}^{p} \ldots \ldots \ldots \ldots \ldots$

3.3 Description of the previous DIS world data set on $g_{1}^{n} \ldots \ldots \ldots$. . . 37

3.4 Description of DIS world data set on $g_{2}^{p}$. . . . . . . . . . . 38

3.5 Description of DIS world data set on $g_{2}^{n}$. . . . . . . . . . . 38

4.1 A listing of the acceptance-averaged kinematics for E97-103 . . . . . 40

4.2 Experimental quantities needed for measurements of $g_{2}^{n} \ldots \ldots \ldots$

5.1 List of energy measurements made for E97-103 . . . . . . . . . . . 52

5.2 The values used for beam polarization $\ldots \ldots \ldots \ldots$

5.3 Table of constants used for calculating accumulated charge sent to the

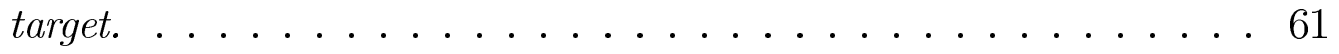

6.1 A list of external cell dimensions and their uncertainties. All values in centimeters. . . . . . . . . . . . . . . . . . . 91

6.2 The Archimedes volume measurements done at the University of Virginia and the College of William and Mary. . . . . . . . . . . 94 
6.3 External Volumes of the cells' chambers. All values in milliliters. . . . 95

6.4 Volume of the three chambers of the $25 \mathrm{~cm}$ water cell. All values are in milliliters. . . . . . . . . . . . . . . . . 95

6.5 Wall thicknesses by section. $A$ is the upstream end, $D$ is the downstream. Left is on the side of the left spectrometer. Right is the on the side of the right spectrometer. All measurements are in centimeters. 97

6.6 The window thicknesses in microns and their window markings. . . . 98

6.7 The estimated chamber volumes in milliliters . . . . . . . . . . . . 99

6.8 A list of the constants used to calibrate the Helmholtz Coils in E97-103.105

6.9 Table of holding field angles given by two surveys and the holding field calibrations. Survey 3 was done just before E97-103 and Survey 4 was done just after. Calibration Angle and Calibration 2 refer to the angle given by the Helmholtz coil currents during Survey 3 and Survey 4 respectively. All angles are given in degrees. . . . . . . . . 108

6.10 Parameters used in extract polarization from NMR signals. . . . . . . 120

6.11 Additional parameters used in water calibrated NMR . . . . . . . . 120

6.12 Values used for the flux calculation. All values are in centimeters . . 122

6.13 Flux values for polarized ${ }^{3}$ He cells. All values are in $\mathrm{cm}^{2}$. . . . . . . 123

6.14 The date, signal heights, the number of sweeps and the flux for each water calibration. . . . . . . . . . . . . 139

6.15 List of Parameters used to calculate $c_{w}^{\prime} \ldots \ldots$. . . . . . . . . 141

6.16 EPR Calibration Constants for NMR. . . . . . . . . . . 146

6.17 List of errors associated with NMR calibration from EPR. . . . . . . 146

6.18 Calibration Constants for NMR signals. Values in Amagats $\mathrm{cm}^{2} / \mathrm{mV} 147$

6.19 List of systematic errors in the polarization measurements in E97-103.147

7.1 Characteristics of the Hall A spectrometers. . . . . . . . . . . 151 
7.2 The electron efficiencies and pion rejection factors for the left and right arm spectrometers. Where $p$ refers to the central electron momentum of the spectrometer. . . . . . . . . . . . . . 161

8.1 The variables used to find good electron events. . . . . . . . . . . . 168

8.2 Sign convention used for asymmetries during E97-103 for the various beam and target polarization combinations. No data was taken in the $180^{\circ}$ target polarization direction in E97-103. . . . . . . . . . . . . 170

8.3 Dilution factors for the 5 kinematic points and the elastic scattering kinematic. Left and Right refer to the Left and Right Spectrometer Arms. . . . . . . . . . . . . . . . . 187

8.4 Tables of the physics asymmetries measured in E97-103. The top table has raw asymmertries which have only been corrected for deadtime and charge. The lower table asymmetries have been corrected for target polarization, beam polarization and dilution factor. . . . . . 190

9.1 A list of materials and their radiation lengths and densities in the incident and scattering electron path. $\phi$ is the central angle of the spectrometer. . . . . . . . . . . . . . . . . 194

9.2 Elastic tails for the five E97-103 kinematic points. All cross-sections are in $n b /(G e V-S r)$. . . . . . . . . . . . . . . 195

9.3 A list of the unpolarized tails from the quasi-elastic cross-section. Both the internal and total (internal + external) contributions are listed. All cross-sections are in units of nb /(GeV-Sr). . . . . . . . . 197

9.4 Internal and total (internal+external) radiative tails from transverse and longitudingal polarized quasi-elastic scattering. All cross-sections

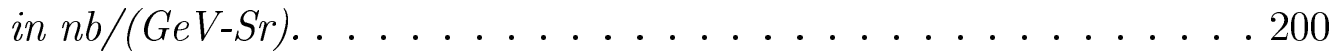

9.5 The longitudinal and transverse inelastic asymmetries. The statistical error and the systematic error resulting from the subtraction are shown.202

9.6 Source of models for structure functions used for the radiative corrections for E97-103. . . . . . . . . . . . . . . . . 204 
9.7 A table of the Born cross-sections from the models of the structure functions and the internal and total (internal+external) radiated crosssections. All cross-sections in $n b /(G e V-S r)$. . . . . . . . . . . . 209

10.1 Values for $g_{2}^{n}$ and $g_{1}^{n}$ and their statistical and systematic error bars. . 216

10.2 Errors on independent variables used to calculate $g_{1}^{n}$ and $g_{2}^{n}$. Percentages are absolute. . . . . . . . . . . . . . . . 218

10.3 Systematic errors on $g_{2}^{n}$ by variable given in percent of $g_{2}^{n}$. . . . . 219

C.1 Comparison of the results for the field direction of the compass survey with the holding field calibration. . . . . . . . . . . . . . . . 224

C.2 Comparison of the results for the field direction of the compass survey with the holding field calibration. . . . . . . . . . . . . . . 2225

C.3 Comparison of the results for the field direction of the compass survey with the holding field calibration. . . . . . . . . . . . . . . 226

C.4 Comparison of the results for the field direction of the compass survey with the holding field calibration. . . . . . . . . . . . . . . . . 227 


\section{LIST OF FIGURES}

2.1 Lowest order Feynman diagram for electron scattering . . . . . . . . . 6

2.2 A qualitative description of an unpolarized structure function $F_{2}^{A}$. . 12

2.3 Coordinate system for polarized elastic scattering. . . . . . . . . 12

2.4 A cartoon description of the ground state and two excited state ${ }^{3} \mathrm{He}$ nucleus polarization configurations. . . . . . . . . . . 16

2.5 Unpolarized ${ }^{3}$ He cross-section . . . . . . . . . . . . . . 17

2.6 $F_{2}^{p}$ from the NMC Collaboration experiments vs. $Q^{2}$ for various values of $x \ldots \ldots \ldots \ldots \ldots \ldots$

3.1 Compton scattering diagrams for twist two and twist three operators 29

3.2 Feynman diagram of a parton with momentum fraction $\xi$ absorbing a virtual photon. . . . . . . . . . . . . . . 32

$3.3 g_{2}^{p}$ from $S L A C E 155 X \ldots \ldots \ldots \ldots \ldots \ldots$

$3.4 g_{2}^{n}$ from SLAC E155X and JLab E99-117 . . . . . . . . . . . . 39

4.1 A schematic of the spectrometer, target and beam line apparatus in Hall A. . . . . . . . . . . . . . . . . . . . . . . . 44

5.1 The electron accelerator facility at Jefferson Lab. . . . . . . . . . . . 47

5.2 A diagram of the helicity feedback system used to control charge asymmetry. . . . . . . . . . . . . . . . 48

5.3 Two diagrams of the source cathode at CEBAF. . . . . . . . . . . . 49 
5.4 The diagram of the equipment used for the Arc energy method. . . . . 50

5.5 A diagram of the eP measurement target and detectors. . . . . . . . . 51

5.6 A diagram of the Møller polarimeter. . . . . . . . . . . . . 53

5.7 Schematic of the Compton apparatus . . . . . . . . . . 55

5.8 Different beam polarization measurements . . . . . . . . . 56

5.9 The results for a comparison of Jefferson Lab beam polarimeters performed in 2000. The polarization is normalized to the Mott polarimeter. The "Hall A Average" is the value of the polarization using the method used in E97-103. . . . . . . . . . . . . . . . . 57

5.10 A plot of the beam helicity in a half second time span as extracted from reconstructed data. See text for details. . . . . . . . . . . . 58

5.11 Beam Charge Montioring system . . . . . . . . . . . 60

5.12 The distribution of charge asymmetry for each run. The left histogram is the charge asymmetry with the beam trips and the right histogram is the charge asymmetry with the beam trips cut out. . . . . . . . . . 64

5.13 A diagram of the beam position monitor . . . . . . . . . . . 65

5.14 The left histogram is a 2-D histogram of the Raster position for a 100000 events. The center histogram is just the $x$ axis distribution for the same 100000 events. The right histogram is the $y$ axis distribution. 66

5.15 The upper-left hand plot is the average $x_{\text {beam }}$ position for the right spectrometer runs for the first kinematic. The upper-right hand plot is the average $y_{\text {beam. }}$. The lower-right hand plot is the average RMS of each run. . . . . . . . . . . . . . . . 67

6.1 A diagram of the polarized ${ }^{3}$ He target system. The transverse Helmholtz coils are not shown for clarity. . . . . . . . . . . . . . 70

6.2 A diagram explaining optical pumping. (1) Rb atoms in a magnetic field are exposed to circularly polarized laser light (2) The valence electron is excited from the ${ }^{5} S_{-1 / 2}$ state to the ${ }^{5} P_{1 / 2}$ state (3) The electron decays by emitting a photon into either the ${ }^{5} S_{-1 / 2}$ where it repeats steps (2) and (3) or into the ${ }^{5} S_{1 / 2}$ state where (4) it remains. 
6.3 The effect of the electron beam on the polarization of a polarized ${ }^{3} \mathrm{He}$

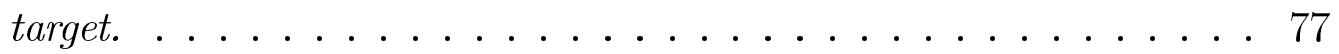

6.4 The electron states of the ${ }^{5} S_{1 / 2}$ orbital of rubidium. . . . . . . . . 78

6.5 Diagram of the glass cell assembly created by the glass-blower. . . . . 80

6.6 Cell Filling system . . . . . . . . . . . . . . . 83

6.7 Cell Baking Configuration . . . . . . . . . . . . . . 84

6.8 A plot of the manifold volume measured during the cell filling process. 87

6.9 Cyrocooling Configuration . . . . . . . . . . . 89

6.10 Names for the cell dimensions . . . . . . . . . . . . . . . . . 91

6.11 The setup for volume measurement using the Archimedes method . . . 92

6.12 Set up for wall thickness measurements at Jefferson Lab . . . . . . . . 96

6.13 The density of the two cells used in E9\%-103. . . . . . . . . . . . . . 99

6.14 Polarizing optics for Jefferson Lab Polarized ${ }^{3}$ He target. . . . . . . . . 101

6.15 A diagram of Laser Optics Configuration in Hall A. . . . . . . . . . . 103

6.16 Helmholtz coils configuration with respect to beam line and lasers. . . 104

6.17 Diagram of frame and probe carriage used to map holding field. . . . . 106

6.18 Compass used to measure the holding field direction. . . . . . . . . . . 107

6.19 Equipment used to power and monitor RF coils. . . . . . . . . . . . . 109

6.20 The $H_{1} R F$ field versus the voltage on the HP Function Generator. $\quad 110$

6.21 The holding field $\vec{H}_{0}$ and rotating field $\vec{H}_{1}$ in the frame of reference defined by $\vec{H}_{e}$ and $\hat{n} \times \vec{H}_{e} \ldots \ldots . \ldots . \ldots . \ldots . \ldots 13$

6.22 Adiabatic Fast Passage . . . . . . . . . . . . . . 116

6.23 The equipment used to monitor NMR signal. . . . . . . . . . . . . 117

6.24 A plot of a signal from a typical NMR measurement. $h$ is the signal height, $H_{1}$ the amplitude of the $R F$ field and $H_{0}$ is the holding field value at the Larmour resonance. . . . . . . . . . . . . . . . . . . . . . 119 
6.25 The reference frame of the magnetic flux calculation with respect to the target chamber and the pick-up coils. . . . . . . . . . . . . 121

6.26 The reference frame of the magnetic flux calculation with respect to the target chamber and the pick-up coils. . . . . . . . . . . . . . 124

6.27 A plot of the helium signal height vs. flux. . . . . . . . . . . . . 124

6.28 The placement of the temperature sensors in E97-103. . . . . . . . . 125

6.29 The external pumping chamber temperatures during NMR measurements in E97-103. . . . . . . . . . . . . . . . . 128

6.30 The relationship between the average reading of RTD 6 and $R T D D^{r}$ and the average internal temperature of the pumping chamber. . . . . . . . 128

6.31 An example of a resonance curve created by the Q-coil. . . . . . . . 129

6.32 Measures of the gain throughout the experiment. . . . . . . . . . . 130

6.33 The effect of the lock-in amplifier time constant on a normalized NMR signal with an $H_{1}$ of $58 m G$. . . . . . . . . . . . . . . . . . . . . 131

6.34 NMR data fit two different ways : a square root of a Lorentzian and one with a shape corrected for time-constant effect and holding field gradients. . . . . . . . . . . . . . . . 132

6.35 The amount of flux from a section of target chamber as a function of $z .133$

6.36 Examples of different sizes of gradients on the ${ }^{3}$ He NMR signal. . . . 134

6.37 The effect on NMR signal height of the longitudinal gradient coil. . . 135

6.38 The effect on NMR signal height of the transverse gradient coil. . . . 135

6.39 A plot of the effects of polarization loss due to AFP during NMR. . . 136

6.40 The magnetic field and $z$ and $x$ of a water cell undergoing AFP. . . . 137

6.41 Average of the 4 water calibrations done in E97-103. . . . . . . . . . 139

6.42 A fit of the Up sweep in the X channel of the 1 Aug 2001 water calibration. . . . . . . . . . . . . . . 140

6.43 Average of the 4 water calibrations done in E97-103. . . . . . . . . . 141 
6.44 The equipment setup for EPR polarimetry. . . . . . . . . . . . . . 143

6.45 The EPR resonance plotted vs. time during AFP. . . . . . . . . . . 144

6.46 The NMR calibration constant from EPR measurements, $c_{\mathrm{EPR}}^{\prime}$. . . . 145

6.47 An example of masing occurring during an EPR measurement. . . . . 148

7.1 A diagram of the Hall A magnets of the high resolution spectrometers. 151

7.2 A diagram of the detector package used in the Hall A spectrometers. . 152

7.3 Two diagrams of the vertical drift chambers. The left diagram shows the path of an electron through the two VDCs. The right diagram shows an electron path firing five wires. . . . . . . . . . . . . . 153

7.4 A plot of the percentage of events assigned to one, two, three and four track events by reconstructing the TDC information from the VDCs. Six runs are plotted for each kinematic, three for the left spectrometer arm and three for the right. The runs were chosen at random from the kinematics. . . . . . . . . . . . . . . . . 155

7.5 A diagram of the scintillator paddles. . . . . . . . . . . . . 156

7.6 A plot of the scintillator efficiencies in the left and right spectrometers. Six runs are chosen for each kinematic, three from the left arm and three from the left arm. The runs are chosen at random. . . . . . 157

7.7 Two diagrams of the gas C̆ernkov detector. The left shows the front of the detector with the cover off. The right is a schematic depicting the mirror collecting Čerenkov light and reflecting it into a phototube. 159

7.8 A diagram of the phototube and lead glass configurations in the preshower, shower and pion rejectors. . . . . . . . . . . . 160

8.1 A flowchart of the various data files and analysis programs used to calculate the charge and dead-time corrected asymmetries. . . . . . . . 164

8.2 A histogram of the ratio of total scaler clock time to event clock time of a run. . . . . . . . . . . . . . . . 167 
8.3 A histogram of the Čerenkov ADC sum for a set of events and a 2-D plot of pre-shower and shower ADC sums. Examples of cuts used in the asymmetry analysis are shown. . . . . . . . . . . . . . 171

8.4 A plot of the effect of different C̆erenkov cuts on the physics asymmetry. The top plot is the effect of different cuts on the four longitudinal configurations and their average. The bottom plot is the effect on the eight transverse configurations and their average. The cuts become tighter from left to right. . . . . . . . . . . . . . . . 172

8.5 A histogram of scattering position along the target cell axis showing the end window cut. . . . . . . . . . . . . . . . . . . . . . . . . 174

8.6 Two plots of the spectrometer acceptance variables. The top plot is of the horizontal scattering angle $\phi$ vs. scattering point along the cell axis, react-z. The bottom plot is of the vertical scattering angle $\theta$ $v s$. the relative momentum difference of the event from the central

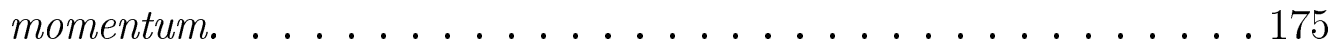

8.7 A study of the effect of tightening cuts along the horizontal scattering axis and the target chamber axis. The dashed line is the value of the asymmetry with the loosest cuts. . . . . . . . . . . . . . 176

8.8 A comparison of the physics asymmetries with different cuts on the minimum current. . . . . . . . . . . . . 177

8.9 A comparison of the physics asymmetries correcting the field alignment with the compass surveys. The $Q^{2}$ values of the fourth survey have been shifted slightly so they can be seen. . . . . . . . . . . . 178

8.10 The asymmetry in the left and right spectrometer arm during carbon quasi-elastic running using charge corrected scaler triggers. . . . . . . 179

8.11 The asymmetry in the left and right spectrometer arm during carbon quasi-elastic running using charge and dead-time corrected detector events. . . . . . . . . . . . . . . . . . 180

8.12 The asymmetry calculated from accepting only events from the glass end windows. . . . . . . . . . . . . . . . . . . 182

8.13 A plot of $W_{\mathrm{N}_{2}}-M_{\mathrm{N}_{2}}$ for a nitrogen reference cell run. . . . . . . . . 183

8.14 A plot of $W_{\mathrm{N}_{2}}-M_{\mathrm{N}_{2}}$ from scattering from a polarized ${ }^{3}$ He cell. . . . . 184 
8.15 The yield from increasing pressures of nitrogen in the reference cell for both the left and right spectrometer arms. . . . . . . . . . . . 185

8.16 The elastic measured in both spectrometers and compared to the simulated value from $S A M C$. . . . . . . . . . . . . . . 188

9.1 Lowest-order electron scattering diagram. . . . . . . . . . . . . . . . 191

9.2 A plot of the Born, internally radiated and externally radiative unpolarized quasi-elastic cross-section. . . . . . . . . . . . . . 196

9.3 Comparison of calculations based on E94-010 data and NQFS Model calculations for the Unpolarized QE Tail. . . . . . . . . . . 197

9.4 Comparison of transverse polarized cross-section from E94-010 and model of the polarized quasi-elastic cross-section. . . . . . . . . . . 198

9.5 Comparison of Transverse Polarized Cross-section from E94-010 and model of the polarized quasi-elastic cross-section. . . . . . . . . . 199

9.6 The longitudinal asymmetries after the elastic and quasi-elastic tails have been subtracted. . . . . . . . . . . . . . . . . 201

9.7 The transverse asymmetries after the elastic and quasi-elastic tails have been subtracted. . . . . . . . . . . . . . . . 201

9.8 The triangles represent the kinematic coverage needed for internal and external radiative corrections for the kinematic point of the right-hand corner of the triangle. . . . . . . . . . . . . . . 203

9.9 The grid used for interpolating the E94-010 data set. . . . . . . . . . 204

9.10 Comparison plots between the E94-010 unpolarized cross-section and cross-section calculated from an interpolation of $F_{2}^{3} \mathrm{He}$. . . . . . . . 206

9.11 Comparison plots between the E94-010 $g_{1}^{3} \mathrm{He}$ and an interpolatiation. Fit stops at pion threshhold. . . . . . . . . . . . . . . . . 207

9.12 Comparison plots between the E94-010 $g_{2}^{3} \mathrm{He}$ and an interpolatiation. Fit stops at pion threshhold. . . . . . . . . . . . . . . . . 208

9.13 Plots of the Born and internally radiated cross-section for the $Q^{2}=$ $0.54 \mathrm{GeV}^{2}$ kinematic. . . . . . . . . . . . . . 210 
9.14 The parallel asymmetries compared with calculations from model. . . . 211

9.15 The perpendicular asymmety compared with calculations from model. . 211

10.1 A comparison of $F_{2}^{3} \mathrm{He}$ interpolated from E94-010 data and calculated from proton and deuterium data from world fits. . . . . . . . . . . . . 213

10.2 The results for $g_{2}^{n}$ and two calculations for $g_{2}^{n} \mathrm{WW}$ from Blülein and Böttcher. . . . . . . . . . . . . . . . . 215

10.3 The results for $g_{1}^{n}$ and two calculations for $g_{1}^{n}$ from the Blülein-Böttcher parton distributions. . . . . . . . . . . . . . . 215

10.4 The results for $g_{2}^{n}$ vs. $x$ plotted with E155X data. . . . . . . . . . . 216

A.1 The calibrations of the small Helmholtz coils. . . . . . . . . . . . . 220

A.2 The calibrations of the large Helmholtz coils. . . . . . . . . . . . . . 221

B.1 Holding field gradient measurements of the small Helmholtz coils. . . . 222

B.2 Holding field gradient measurements for the large Helmholtz coils. . 223 


\begin{abstract}
Jefferson Lab experiment E97-103 measured the spin structure function $g_{2}^{n}\left(x, Q^{2}\right)$ from a $Q^{2}$ of 0.58 to 1.36 with a nearly constant $x$ of 0.2 . Combining this data with a fit to the world $g_{1}^{n}$ data, the size of higher twist contributions to the spin structure functions can be extracted using the Wandzura-Wilczek relation. These higher twist contributions result from quark-gluon correlations and are expected to be larger as $Q^{2}$ decreases. This experiment was performed in Hall A with a longitudinally polarized electron beam and a high density polarized ${ }^{3} \mathrm{He}$ target. The physics motivation and an overview of the experiment will be presented.
\end{abstract}


THE SEARCH FOR HIGHER TWIST EFFECTS IN THE NEUTRON SPIN STRUCTURE FUNCTION $g_{2}^{n}\left(x, Q^{2}\right)$ 


\section{CHAPTER 1}

\section{Introduction}

The first evidence that the nucleon has substructure was the measurement by Esterman and Stern [1][2] of the magnetic moments of the proton and the neutron. Because the electron and proton have the same magnitude of electric charge the magnetic moment of the proton, corrected for the mass, was also expected to be the same in magnitude as that of the electron. The measured moment turned out to be 2.7 times larger. Moreover, the neutron is electrically neutral and was expected to have no magnetic moment at all, but was instead found to have a magnetic moment almost twice as large as that of the electron! Consequently, one had to conclude that the charge in the nucleon was not point-like, but distributed in space in such a way that some (in the case of the proton) or all (in the case of the neutron) of the

electric fields cancelled. Seventy years have passed since this experiment and there is still much to be learned about the charge distributions of the nucleon.

In the mid-1950s, McAllister and Hofstadter [3] began the first electron scattering experiments to study nucleon (meaning proton or neutron) structure. They found that the nucleon was indeed an extended object and many theories were constructed to describe what the nucleon was made of and what held it together. With Feynman's formulation of the parton model [4] and its subsequent confirmation by SLAC experiments in the late 1960's and early 1970's, the correct degrees of freedom for the nucleon became clear. These were interpreted to be the quarks and gluons proposed by Gell-Mann [5] and Zweig [6] in their efforts to explain hadron mass spectroscopy. 
The relativistic theory describing the interaction of quarks by exchange of gluons, and therefore the structure of the nucleon, become known as quantum chromodynamics (QCD). QCD is still the considered the correct theory of nucleon physics, but because it is a non-Abelian theory it is has been difficult to describe the properties of the nucleon using QCD formalism. The challenge of today's physicists studying nucleon structure is to untangle the more difficult aspects of QCD through experiments and theoretical insight so that a clearer picture of the nucleon and the strong interaction can emerge.

In the 1980's people became interested in using spin degrees of freedom to understand nucleon structure, as Estermann and Stern had used charge properties. Physicists developed new polarized beams and polarized targets to study how the total spin of the nucleon was distributed among the quarks and gluons. This work, which began at SLAC and continued at CERN, DESY and Jefferson Lab, showed that the study of nucleon spin structure provided a wealth of information about QCD and nucleon structure.

The experiment described by this dissertation, Jefferson Lab experiment E97103 , follows in the tradition of these experiments. It uses spin-dependent quantities to isolate and quantify the magnitude of quark-gluon interactions, known as highertwist effects. It is hoped that a precise measurement of these higher twist effects will be a signficant step in understanding nucleon structure through QCD. 


\section{CHAPTER 2}

\section{Inclusive Electron Scattering}

\subsection{Introduction}

There are many reasons that inclusive electron scattering is a useful tool for studying nucleon structure. First, electrons can interact with hadronic material only through the electromagnetic and weak interactions (gravity is, of course, negligible between small masses). This allows the electrons to probe the entire charge distribution of the nucleon or nucleus structure, unlike hadron-hadron scattering which typically probes only the surfaces of the hadronic material. Furthermore, electron scattering is dominated by the one-photon exchange electromagnetic interaction. This is fortunate since this ensures that the electron survives the scattering process and scatters in an uncomplicated manner.

In inclusive electron scattering, only the scattered electron is detected which means it is insensitive to any specific reactions and measures the scattering from all possible interactions. In exclusive or semi-inclusive scattering, one detects the scattered electron along with some or all of the other particles produced, which allows one to separate out specific reaction channels. Inclusive scattering has the experimental advantage of only requiring the detection of the scattered electrons. Unfortunately, it has the disadvantage that there is ambiguity about which interaction the electron underwent. Nevertheless, one-photon exchange dominates the cross-section and accurate measurements of the nucleon substructure are possible with some usually moderate corrections. 


\subsection{The Framework of Polarized Inclusive Electron Scattering}

\subsubsection{Kinematic Variables}

In inclusive electron scattering experiments, the structure of a target nucleon or nucleus is described in terms of a differential cross section from the process

$$
l(k)+N(p) \longrightarrow l\left(k^{\prime}\right)+X\left(p^{\prime}\right)
$$

where $l(k)$ is an electron with four-momentum $k, N(p)$ is a nucleon or nucleus with four-momentum $p$ and $X\left(p^{\prime}\right)$ is the hadronic final state with momentum $p^{\prime}$. In inclusive electron scattering, all hadronic final states are included in the cross section. A Feynman diagram for this process is shown in Fig. 2.1. The weak interaction, though present, is small in comparison to the electromagnetic interaction and is ignored for this discussion.

Before writing the cross section for this process it is useful to describe some basic variables. Table 2.1 is a list of the most common kinematic variables used in parameterizing electron scattering. Table 2.2 is a list of invariant parameters.

In general, the structure functions (which will be mentioned later in the chapter) are parameterized in terms of $Q^{2}$ and $x$. This is because at high $Q^{2}$ the structure functions vary slowly with $Q^{2}$ and can be considered a function only of $x$ (this is known as Bjorken scaling and will be discussed later in the chapter). However, it is often useful to present the structure functions as functions of $Q^{2}$ and $W$. This is because certain features of the cross-section, (such as the pion threshold, elastic and quasi-elastic scattering and the peak of the $\Delta$ resonance) take place at fixed values

of $W$. Its also not unusual to see the structure functions in terms of $Q^{2}$ and $\nu$ since $\nu$ is a convenient variable for experimentalists.

The mass term $M$ is the mass of the proton (even when describing a neutron cross-section) corresponding to the structure function measured. In the case of nuclear elastic scattering, the mass of the entire nucleus is represented by $M_{T}$. 


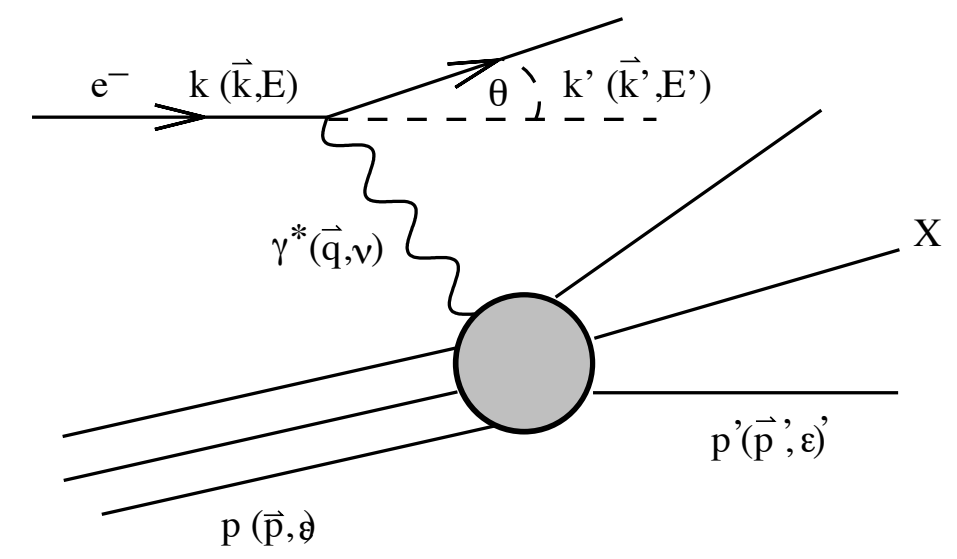

Figure 2.1: Lowest order Feynman diagram for electron scattering on a fixed target.

\begin{tabular}{ccl}
\hline \hline Parameter & Expression & Description \\
\hline$\varepsilon$ & & initial target energy \\
$\varepsilon^{\prime}$ & & scattered target energy \\
$p$ & $(M, \overrightarrow{0})$ & initial target 4-momentum (for a fixed target) \\
$E$ & & incident electron energy \\
$E^{\prime}$ & & scattered electron energy \\
$\nu$ & $E-E^{\prime}$ & energy transfer \\
$k$ & $(E, \vec{k})$ & incident electron 4-momentum \\
$k^{\prime}$ & $\left(E^{\prime}, \overrightarrow{k^{\prime}}\right)$ & scattered electron 4-momentum \\
$\theta$ & & electron scattering angle \\
$q$ & $k-k^{\prime}$ & 4-momentum transfer \\
\hline
\end{tabular}

Table 2.1: A table of important kinematic factors expressed in the lab frame.

\begin{tabular}{cccl}
\hline \hline Parameter & $\begin{array}{c}\text { Invariant } \\
\text { Expression }\end{array}$ & $\begin{array}{c}\text { Lab Frame } \\
\text { Expression }\end{array}$ & Description \\
\hline$Q^{2}$ & $-q^{2}$ & $4 E E^{\prime} \sin ^{2}\left(\frac{\theta}{2}\right)$ & four-momentum transfer squared \\
$W^{2}$ & $M^{2}+p \cdot q+q^{2}$ & $M^{2}+2 M \nu-Q^{2}$ & $\begin{array}{l}\text { squared invariant mass of resulting } \\
\text { target system X }\end{array}$ \\
$x$ & $\frac{Q^{2}}{2 p \cdot q}$ & $\frac{Q^{2}}{2 M \nu}$ & Bjorken scaling variable \\
$y$ & $\frac{q \cdot p}{k \cdot p}$ & $\frac{\nu}{E}$ & fraction of energy loss \\
\hline
\end{tabular}

Table 2.2: A table of important kinematic factors expressed in terms of invariants and in terms of lab frame parameters. 


\subsubsection{Deriving the differential cross-section}

The differential cross-section can be written in terms of a scattering matrix $T_{f i}$ and kinematic factors [7]

$$
d \sigma=\frac{\overline{\left|T_{f i}\right|^{2}}}{\mathcal{J}} \frac{d^{3} k^{\prime}}{2 E^{\prime}(2 \pi)^{3}} \frac{d^{3} p^{\prime}}{2 \varepsilon^{\prime}(2 \pi)^{3}}(2 \pi)^{4} \delta^{4}\left(k-k^{\prime}+p-p^{\prime}\right)
$$

where $\mathcal{J}=4 p \cdot k$ which reduces to $4 M \varepsilon$ in the lab frame. The scattering matrix can be written :

$$
T_{f i}=\frac{e^{2}}{q^{2}}\left\langle l\left(k^{\prime}\right)\left|j^{\mu}(0)\right| l(k)\right\rangle\left\langle X\left(p^{\prime}\right)\left|J_{\mu}(0)\right| N(p)\right\rangle
$$

where $j^{\mu}$ and $J^{\mu}$ are the leptonic and quark electromagnetic currents as given by the Standard Model $[8,9,10]$. Squaring this matrix gives :

$$
\overline{\left|T_{f i}\right|^{2}}=\frac{e^{4}}{q^{4}} L_{\mu \nu} W^{\mu \nu}
$$

where $L_{\mu \nu}$ and $W^{\mu \nu}$ represent the leptonic and hadronic vertex tensors respectively. One can then write the differential cross-section as

$$
\frac{d^{2} \sigma}{d \Omega d E^{\prime}}=\frac{\alpha^{2}}{q^{4}} \frac{E^{\prime}}{E} L_{\mu \nu} W^{\mu \nu}
$$

where $\alpha=e^{2} / 4 \pi$.

\subsubsection{The leptonic and hadronic tensors}

It is helpful that these tensors can be separated, since $L_{\mu \nu}$ is well-known from quantum electrodynamics (QED) [7]

$$
\begin{aligned}
L_{\mu \nu} & =\sum_{s^{\prime}} \bar{u}_{s}(k) \gamma_{\mu} u_{s^{\prime}}\left(k^{\prime}\right) \bar{u}_{s^{\prime}}\left(k^{\prime}\right) \gamma_{\nu} u_{s}(k) \\
& =2\left[k_{\mu} k_{\nu}^{\prime}+k_{\mu}^{\prime} k_{\nu}-g_{\mu \nu} k \cdot k^{\prime}+i \epsilon_{\mu \nu \alpha \beta} s^{\alpha} q^{\beta}\right]
\end{aligned}
$$

where $s_{\mu}=\bar{u} \gamma_{\mu} \gamma_{5} u$ is the lepton spin vector and the electron mass is neglected.

The hadronic vertex tensor must contain all possible transitions from the nucleon or nucleus into any possible excited state [7]. This term is where the strong 
interactions appear in the cross section. A general form of this vertex can be written

$$
W_{\mu \nu}(P, q)=\frac{1}{2 M} \sum_{X}\left\langle N_{s}(p)\left|J_{\mu}(0)\right| X\left(p^{\prime}\right)\right\rangle\left\langle X\left(p^{\prime}\right)\left|J_{\nu}(0)\right| N_{s}(p)\right\rangle \cdot(2 \pi)^{3} \delta^{4}\left(q+p-p^{\prime}\right)
$$

where the initial nucleon spin state is denoted by $s= \pm \frac{1}{2}$. This tensor can be presented in a form using the completeness of the states $|X\rangle$ :

$$
W_{\mu \nu}=\frac{1}{4 \pi M} \int d^{4} z e^{i q \cdot z}\left\langle N_{s}(p)\left|J_{\mu}(z) J_{\nu}(0)\right| N_{s}(p)\right\rangle
$$

or it can be written in terms of a current commutator as

$$
W_{\mu \nu}=\frac{1}{4 \pi M} \int d^{4} z e^{i q \cdot z}\left\langle N_{s}(p)\left|\left[J_{\mu}(z), J_{\nu}(0)\right]\right| N_{s}(p)\right\rangle
$$

where the extra term, $J_{\nu}(0) J_{\mu}(z)$, gives a vanishing matrix element since it produces a delta function $\delta^{4}\left(q-p+p^{\prime}\right)$ that cannot be satisfied [7].

The hadronic tensor has separable symmetric and antisymmetric parts as shown here:

$$
W_{\mu \nu}=W_{\mu \nu}^{S}+W_{\mu \nu}^{A}
$$

The most general forms of these tensors, as limited by gauge invariance and parity conservation of the electromagnetic interaction, are:

$$
W_{\mu \nu}^{S}=W_{1}\left(\nu, Q^{2}\right)\left(\frac{q_{\mu} q_{\nu}}{q^{2}}-g_{\mu \nu}\right)+\frac{W_{2}\left(\nu, Q^{2}\right)}{M^{2}}\left(p_{\mu}-\frac{p \cdot q}{q^{2}} q_{\mu}\right)\left(P_{\nu}-\frac{P \cdot q}{q^{2}} q_{\nu}\right)
$$

and

$$
W_{\mu \nu}^{A}=i \epsilon_{\mu \nu \alpha \beta} q^{\alpha}\left[G_{1}\left(\nu, Q^{2}\right) S^{\beta}+\frac{G_{2}\left(\nu, Q^{2}\right)}{M^{2}}\left(S^{\beta} p \cdot q-p^{\beta} S \dot{q}\right)\right]
$$

where $W_{1}\left(\nu, Q^{2}\right)$ and $W_{2}\left(\nu, Q^{2}\right)$ are the unpolarized response functions, $G_{1}\left(\nu, Q^{2}\right)$ and $G_{2}\left(\nu, Q^{2}\right)$ are the polarized response functions and $S^{\mu}=\bar{u}(p) \gamma^{\mu} \gamma_{5} u(p) / 2 M$ is the nucleon spin vector. Thus the physics of the nucleon structure that is accessible in inclusive lepton scattering is all encapsulated in $W_{1}, W_{2}, G_{1}$ and $G_{2}$. 


\subsubsection{The structure functions}

Normally, the cross section is not described in terms of the response function, but a set of structure functions displayed here:

$$
\begin{aligned}
& F_{1}\left(x, Q^{2}\right)=M W_{1}\left(\nu, Q^{2}\right), \\
& F_{2}\left(x, Q^{2}\right)=\nu W_{2}\left(\nu, Q^{2}\right), \\
& g_{1}\left(x, Q^{2}\right)=M \nu G_{1}\left(\nu, Q^{2}\right), \\
& g_{2}\left(x, Q^{2}\right)=\nu^{2} G_{2}\left(\nu, Q^{2}\right) .
\end{aligned}
$$

From these structure functions, the relevant forms of the differential cross section can be written.

For the case of an unpolarized beam and target the differential cross section written in terms of lab frame parameters is

$$
\frac{d^{2} \sigma}{d \Omega d E^{\prime}}=\frac{\alpha^{2}}{4 E^{2} \sin ^{4} \frac{\theta}{2}}\left(\frac{F_{2}\left(x, Q^{2}\right)}{\nu} \cos ^{2} \frac{\theta}{2}+\frac{2 F_{1}\left(x, Q^{2}\right)}{M} \sin ^{2} \frac{\theta}{2}\right) .
$$

It is of additional interest that $F_{1}\left(x, Q^{2}\right)$ and $F_{2}\left(x, Q^{2}\right)$ are related through the function $R\left(x, Q^{2}\right)$ defined as:

$$
R\left(x, Q^{2}\right) \equiv \frac{\sigma_{L}}{\sigma_{R}}
$$

where $\sigma_{L}$ and $\sigma_{R}$ are the longitudinal and transverse virtual photo-absorption cross sections [11][12]. The relation between $F_{1}\left(x, Q^{2}\right)$ and $F_{2}\left(x, Q^{2}\right)$ is:

$$
F_{1}\left(x, Q^{2}\right)=\frac{1+\frac{Q^{2}}{\nu^{2}}}{2 x\left[1+R\left(x, Q^{2}\right)\right]} F_{2}\left(x, Q^{2}\right) .
$$

The only spin-dependent terms that survive in the cross-sections are ones that have both electron and target polarization. The electron beam can either be polarized along or opposite to the direction of the electron beam path. The electron beam can be polarized perpendicular to the beam path, but that is not relevant to the physics presented in this thesis. The target polarization can either be in the direction of the electron beam, referred to as longitudinal polarization or perpendicular to the electron beam, referred to as transverse polarization. 
The spin-dependent part of the electron scattering cross-section for a longitudinally polarized cross-section is

$$
\begin{aligned}
\left(\frac{d^{2} \sigma}{d \Omega d E^{\prime}}\right)_{\text {long.pol. }} & =\frac{1}{2}\left(\frac{d^{2} \sigma^{\downarrow \uparrow}}{d \Omega d E^{\prime}}-\frac{d^{2} \sigma^{\uparrow \Uparrow}}{d \Omega d E^{\prime}}\right) \\
& =\frac{2 \alpha^{2} E^{\prime}}{Q^{2} E M \nu}\left[\left(E+E^{\prime} \cos \theta\right) g_{1}\left(x, Q^{2}\right)-2 x M g_{2}\left(x, Q^{2}\right)\right]
\end{aligned}
$$

where $\uparrow$ and $\downarrow$ refer to the direction of the electron beam polarization, either along or opposite the beam path, and $\Uparrow$ means a target polarized along the beam direction. When the target is transversely polarized, the spin-dependent part of the cross section can be written

$$
\begin{aligned}
\left(\frac{d^{2} \sigma}{d \Omega d E^{\prime}}\right)_{\text {trans.pol. }} & =\frac{1}{2}\left(\frac{d^{2} \sigma^{\downarrow \Leftarrow}}{d \Omega d E^{\prime}}-\frac{d^{2} \sigma^{\uparrow \Leftarrow}}{d \Omega d E^{\prime}}\right) \\
& =\frac{2 \alpha^{2} E^{\prime}}{Q^{2} E M \nu} E^{\prime} \sin \theta\left[g_{1}\left(x, Q^{2}\right)+\frac{4 x E M}{Q^{2}} g_{2}\left(x, Q^{2}\right)\right]
\end{aligned}
$$

where $\Leftarrow$ refers to a transversely polarized target. Both polarized cross sections change sign when the target is polarized in the opposite direction (i.e. when the target is polarized opposite the beam direction or transversely polarized at $90^{\circ}$ rather than $270^{\circ}$ when the beam direction is $0^{\circ}$ ).

\section{$\underline{2.3}$ Types of inclusive electron scattering}

\subsubsection{General description of electron scattering on a nucleus}

The cross-sections described by equations $2.18,2.21$ and 2.23 are quite general and are good for all $x$ and $Q^{2}$; however, it is useful to separate different kinematic regions from one another for analysis purposes. The three scattering types that will be described are elastic, quasi-elastic and inelastic.

Elastic scattering occurs in the region where energy transfer $(\nu)$ from the electron to target is not large enough to excite the target into higher nuclear or hadronic states, but simply accelerates the nucleus to a new momentum. This means for a 
given incident electron energy and scattering angle there is a unique electron scattering momentum. In Fig. 2.2, one can see that the elastic peak in the structure function $F_{2}^{A}$ (the superscript $A$ referes to the number of nucleons in the nucleus) is a delta function in $x$ for a single $Q^{2}$. Excited nuclear states occur in $F_{2}^{A}$ when energy from the probing electron creates a higher-energy configuration of the target nucleus.

Quasi-elastic scattering is elastic scattering off a nucleon (proton or neutron) that is part of a nucleus. It can be approximated as elastic scattering off a nucleon that has some Fermi motion (due to the nuclear binding). This Fermi motion causes the peak to be distributed in $x$ as can be seen in Fig. 2.2 [13].

The resonance region begins where the energy transfer is large enough to create new hadrons. The lower limit is defined at the pion threshhold since pions are the lightest hadrons. At high energy and momentum transfer $(W>2.0 \mathrm{GeV})$ the probing electron behaves as if it were only scattering off a point-like quark. This is known as deep inelastic scattering.

\subsubsection{Polarized elastic scattering on the nucleon}

Elastic scattering can be seen as a special case of Eq. 2.18 where $W^{2}=M^{2}$ which implies $\nu=Q^{2} / 2 M$. Because of this the scattered electron energy for a certain beam energy and scattering angle in the lab frame will always be

$$
E^{\prime}=\frac{E}{1+\frac{2 E}{M} \sin ^{2} \frac{\theta}{2}}
$$

This means that cross sections will be expressed in terms of $\frac{d \sigma}{d \Omega}$ instead of $\frac{d \sigma}{d E^{\prime} d \Omega}$.

The usual formalism of polarized elastic scattering introduces a vector $\vec{S}_{A}$ to describe the unit target polarization direction as shown in Fig. 2.3 and written [14]

$$
\vec{S}_{A}=\hat{x} \cos \phi^{*} \sin \theta^{*}+\hat{y} \sin \phi^{*} \sin \theta^{*}+\hat{z} \cos \theta^{*}
$$

where $\theta^{*}$ and $\phi^{*}$ are the spherical coordinates of the momentum transfer vector $\vec{q}$. 


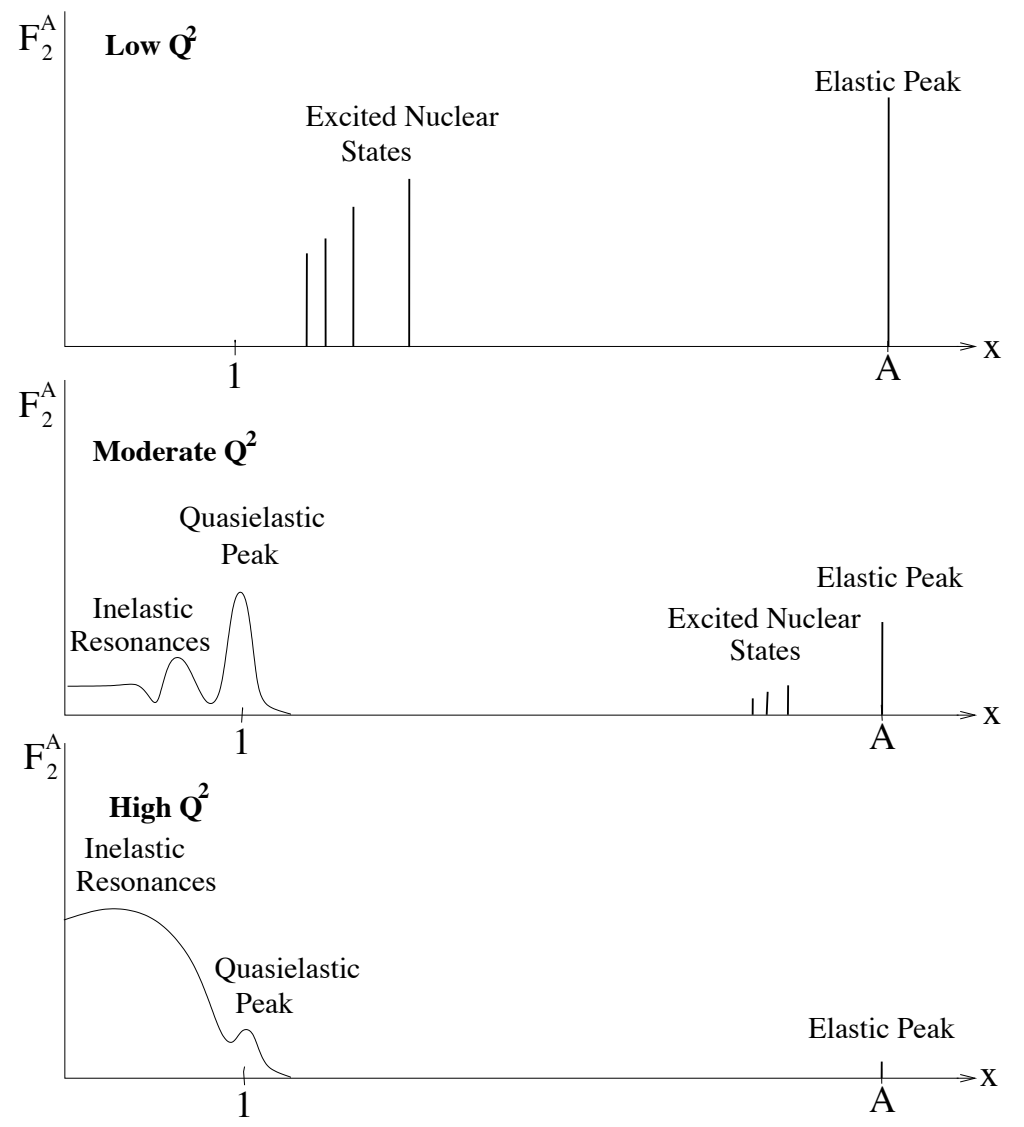

Figure 2.2: A qualitative description of the unpolarized structure function $F_{2}^{A}$ of a nucleus with A nucleons as a function of Bjorken $x$ for different $Q^{2}$.

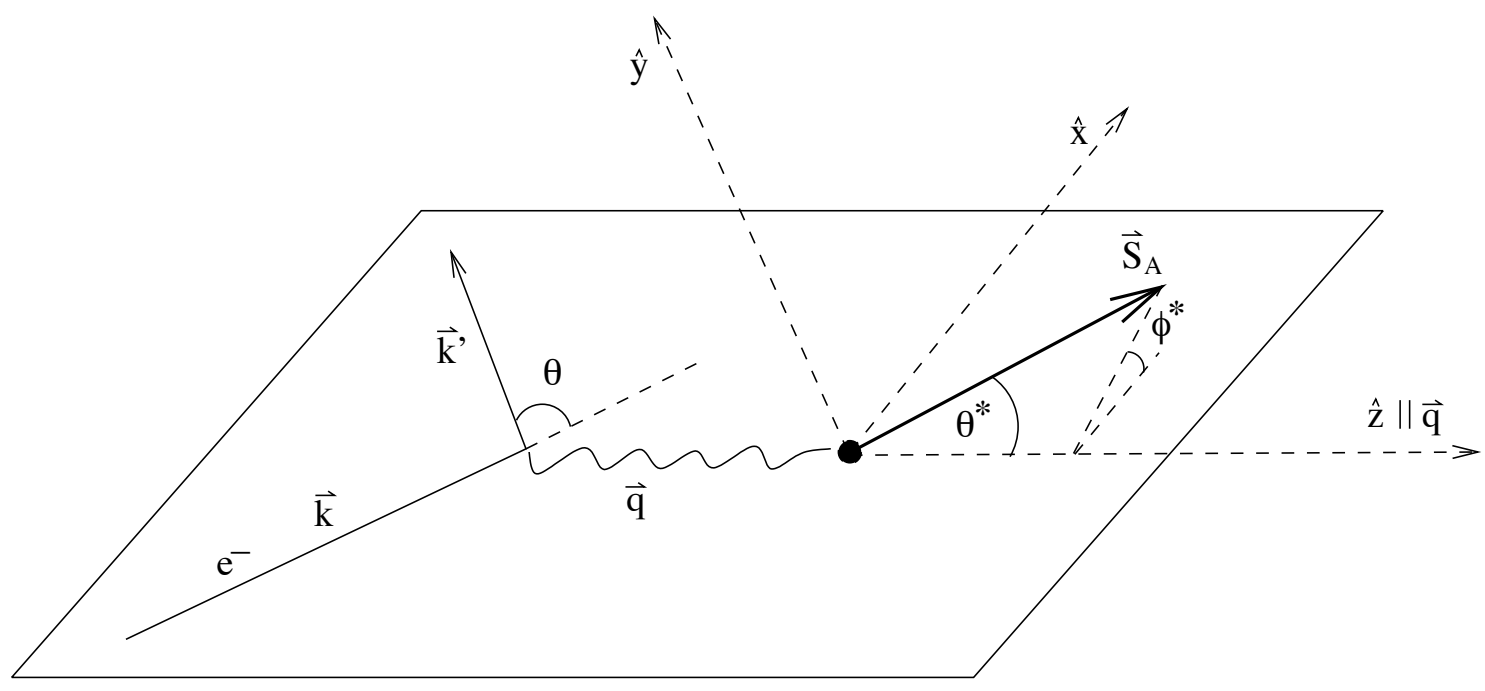

Figure 2.3: Coordinate system for polarized elastic scattering. 
Substituting this polarization vector in for $S$ and assuming $W^{2}=M^{2}$ one can make the following substitutions into Eqs. 2.12 and 2.13 [15]:

$$
\begin{aligned}
W_{1}\left(\nu, Q^{2}\right) & =\frac{1}{2} R_{T}\left(Q^{2}\right) \delta\left(\nu-\frac{Q^{2}}{2 M}\right) \\
W_{2}\left(\nu, Q^{2}\right) & =\frac{R_{L}\left(Q^{2}\right)+\frac{1}{2} R_{T}\left(Q^{2}\right)}{1+\tau} \delta\left(\nu-\frac{Q^{2}}{2 M}\right) \\
G_{1}\left(\nu, Q^{2}\right) & =\frac{1}{2} \frac{M^{2}}{Q^{2}}\left(\frac{Q^{2}}{M\left|\overrightarrow{q^{2}}\right|} \frac{R_{T L^{\prime}}\left(Q^{2}\right)}{\sqrt{2}}\right) \delta\left(\nu-\frac{Q^{2}}{2 M}\right) \\
G_{2}\left(\nu, Q^{2}\right) & =-\frac{1}{2} \frac{M^{2}}{Q^{2}}\left(\frac{\nu}{|\vec{q}|} \frac{R_{T L^{\prime}}\left(Q^{2}\right)}{\sqrt{2}}+R_{T^{\prime}}\left(Q^{2}\right)\right) \delta\left(\nu-\frac{Q^{2}}{2 M}\right)
\end{aligned}
$$

where

$$
\begin{aligned}
|\vec{q}|^{2} & =\nu^{2}+Q^{2} \\
\tau & =\frac{Q^{2}}{4 M^{2}}
\end{aligned}
$$

and where $R_{L}, R_{T}, R_{T L^{\prime}}$ and $R_{T^{\prime}}$ are the elastic response functions that depend on the type of target. For nucleon scattering the response functions are written in terms of the elastic and magnetic form factors $G_{E}$ and $G_{M}$ :

$$
\begin{aligned}
R_{T}\left(Q^{2}\right) & =(1+\tau) G_{E}^{2}\left(Q^{2}\right) \\
R_{L}\left(Q^{2}\right) & =2 \tau G_{M}^{2}\left(Q^{2}\right) \\
R_{T^{\prime}}\left(Q^{2}\right) & =2 \tau G_{M}^{2}\left(Q^{2}\right) \\
R_{T L^{\prime}}\left(Q^{2}\right) & =-\sqrt{2 \tau(1+\tau)} G_{E}\left(Q^{2}\right) G_{M}\left(Q^{2}\right) .
\end{aligned}
$$

The differential cross section for unpolarized elastic scattering in the lab frame can be written

$$
\left(\frac{d \sigma}{d \Omega}\right)_{\text {unpol. }}=\left(\frac{d \sigma}{d \Omega}\right)_{\text {Mott }}\left[\frac{G_{E}^{2}\left(Q^{2}\right)+\tau G_{M}^{2}\left(Q^{2}\right)}{1+\tau}+2 \tau G_{M}^{2}\left(Q^{2}\right) \tan ^{2} \frac{\theta}{2}\right]
$$

where

$$
\left(\frac{d \sigma}{d \Omega}\right)_{\text {Mott }}=\frac{\alpha^{2} \cos ^{2} \frac{\theta}{2}}{4 E^{2} \sin ^{4} \frac{\theta}{2}\left(1+\frac{2 E}{M} \sin ^{2} \frac{\theta}{2}\right)} .
$$


The differential cross section for polarized elastic scattering in the lab frame can be expressed as

$$
\left(\frac{d \sigma}{d \Omega}\right)_{\text {pol. }}=-h\left(\frac{d \sigma}{d \Omega}\right)_{\text {Mott }}\left[\cos \theta^{*} v_{T^{\prime}} R_{T^{\prime}}\left(Q^{2}\right)+2 \sin \theta^{*} \cos \phi^{*} v_{T L^{\prime}} R_{T L^{\prime}}\left(Q^{2}\right)\right]
$$

where

$$
\begin{aligned}
v_{T^{\prime}} & =\tan \frac{\theta}{2} \sqrt{\frac{Q^{2}}{|\vec{q}|^{2}}+\tan ^{2} \frac{\theta}{2}} \\
v_{T L^{\prime}} & =-\frac{1}{\sqrt{2}}\left(\frac{Q^{2}}{|\vec{q}|^{2}}\right) \tan \frac{\theta}{2}
\end{aligned}
$$

where $h$ is the incident electron helicity and is equal to either +1 or -1 . To get a longitudinal and transverse target comparable to those in Eq. 2.21 and Eq. 2.23, one must set $\theta^{*}$ to the angle between the target polarization and the $\vec{q}$ direction. Because this experiment only dealt with in-plane scattering, $\phi^{*}$ is always 0 or $\pi$ here.

\subsubsection{Polarized elastic scattering on ${ }^{3} \mathrm{He}$}

The previous section described elastic scattering on a nucleon which, in principle, applies to the neutron as well as the proton. However, the form factors from the neutron must be extracted from nuclear targets since no practical pure neutron target exists. Similarly, the structure functions for the neutron must be extracted from nuclear targets. This experiment extracts the neutron structure functions from polarized ${ }^{3} \mathrm{He}$; therefore, it is useful to describe the formalism for polarized ${ }^{3} \mathrm{He}$ elastic scattering.

The unpolarized cross section for elastic scattering on ${ }^{3} \mathrm{He}$ [16]:

$$
\left(\frac{d \sigma}{d \Omega}\right)_{\text {unpol. }}=Z^{2} \frac{1}{\eta}\left(\frac{d \sigma}{d \Omega}\right)_{\text {Mott }}\left[\frac{Q^{2}}{|\vec{q}|^{2}} F_{c}^{2}\left(Q^{2}\right)+\frac{\mu^{2} Q^{2}}{2 M_{T}^{2}}\left(\frac{1}{2} \frac{Q^{2}}{|\vec{q}|^{2}}-\tan ^{2} \frac{\theta}{2}\right) F_{m}^{2}\left(Q^{2}\right)\right]
$$

where $Z$ is the charge of the nucleus, $\mu$ is the magnetic moment, $\eta=1-Q^{2} / 4 M_{T}^{2}$ is a factor taking into account the target recoil and $M_{T}$ is the mass of the ${ }^{3} \mathrm{He}$ 
nucleus. $F_{c}\left(Q^{2}\right)$ and $F_{m}\left(Q^{2}\right)$ are the charge and magnetic form factors for ${ }^{3} \mathrm{He}$ and are analogous to $G_{E}\left(Q^{2}\right)$ and $G_{M}\left(Q^{2}\right)$ for the nucleon.

The polarized cross section for polarized ${ }^{3}$ He follows the same form as Eq. 2.39 except with new response functions:

$$
\begin{aligned}
R_{T^{\prime}}\left(Q^{2}\right) & =\frac{2 \tau E^{\prime}}{E}\left(\mu_{A} F_{m}\right)^{2} \\
R_{T L^{\prime}}\left(Q^{2}\right) & =-\frac{E^{\prime} \sqrt{2 \tau(1+\tau)}}{E}\left(Z F_{c}\right)\left(\mu_{A} F_{m}\right)
\end{aligned}
$$

where $\mu_{A}$ is the magnetic moment of the nucleus.

\subsubsection{The effective polarization of the neutron in the ${ }^{3} \mathrm{He}$ nucleus}

Before describing the details of polarized quasi-elastic and inelastic scattering, one must confront a complication resulting from use of a nucleus rather than a nucleon as a target. To be able to extract information about the nucleon from polarized nuclei, one must understand what fraction of the total nuclear polarization comes from each nucleon.

In a polarized ${ }^{3}$ He nucleus, almost all the polarization comes from the neutron. The Pauli exclusion principle ensures that the polarization from the two protons cancel each other in the lowest energy state of the nucleus. Of course, the nucleus has excited states due to nucleon-nucleon interactions. The most important higher states are shown in Fig. 2.4.

Calculations of the effective polarization of a nucleon based on different models of the ${ }^{3} \mathrm{He}$ nucleus has been done by several groups and is summarized by Bissey et al. $[17]$.

\subsubsection{Polarized quasi-elastic scattering on ${ }^{3} \mathrm{He}$}

A rough approximation of the quasi-elastic scattering cross-section of the nucleus is the sum of the elastic scattering from each nucleon in the nucleus. However, 


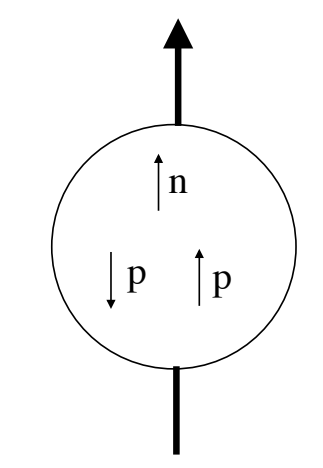

S (Ground) state

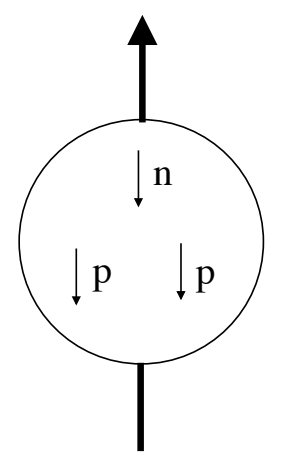

D state

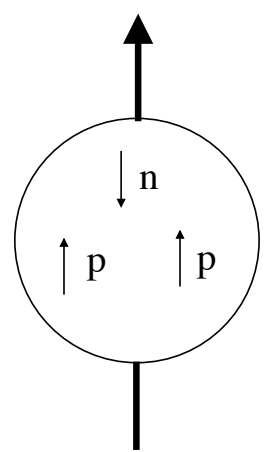

S' state

Figure 2.4: A cartoon description of the ground state and two excited state ${ }^{3}$ He nucleus polarization configurations.

effects like Fermi motion, final state interactions and meson exchange distort the elastic scattering picture.

One description of the cross section for unpolarized quasi-elastic scattering comes from a model by Lightbody and O'Connell [18]. In this description, the quasi-elastic cross section is represented by a Gaussian peak written as

$$
\sigma_{q e}^{3} \mathrm{He}\left(\nu, Q^{2}\right)=A e^{-\frac{\left(\nu-Q^{2} / 2 M\right)}{2|\overline{\mid}|^{2} k_{F}^{2} / M^{2}}}
$$

where $k_{F}$ is the Fermi momentum of the nucleus and the constant $A$ is defined by the relation

$$
2 \sigma_{q e}^{p}+\sigma_{q e}^{n}=\int_{0}^{E_{\mathrm{beam}}} A e^{-\frac{\left(\nu-Q^{2} / 2 M\right)}{2|q|^{2} k_{F}^{2} / M^{2}}} d \nu
$$

where $\sigma_{q e}^{p}$ and $\sigma_{q e}^{n}$ are the elastic cross sections for the proton and neutron respectively with form factors modified to fit quasi-elastic data.

The polarized quasi-elastic cross-section is more complicated, but in principle can be derived from similar techniques (i.e. broadening modified elastic cross sections into a Gaussian peak). A straight-forward description by Leidemann et al [19], uses the elastic formalism with the following substitutions :

$$
R^{T^{\prime}}\left(Q^{2}\right)=-2 \tau\left\{G_{M}^{n 2}\left(Q^{2}\right)-\frac{2}{3} P_{D}\left[G_{M}^{p 2}\left(Q^{2}\right)+2 G_{M}^{n 2}\left(Q^{2}\right)\right]\right.
$$




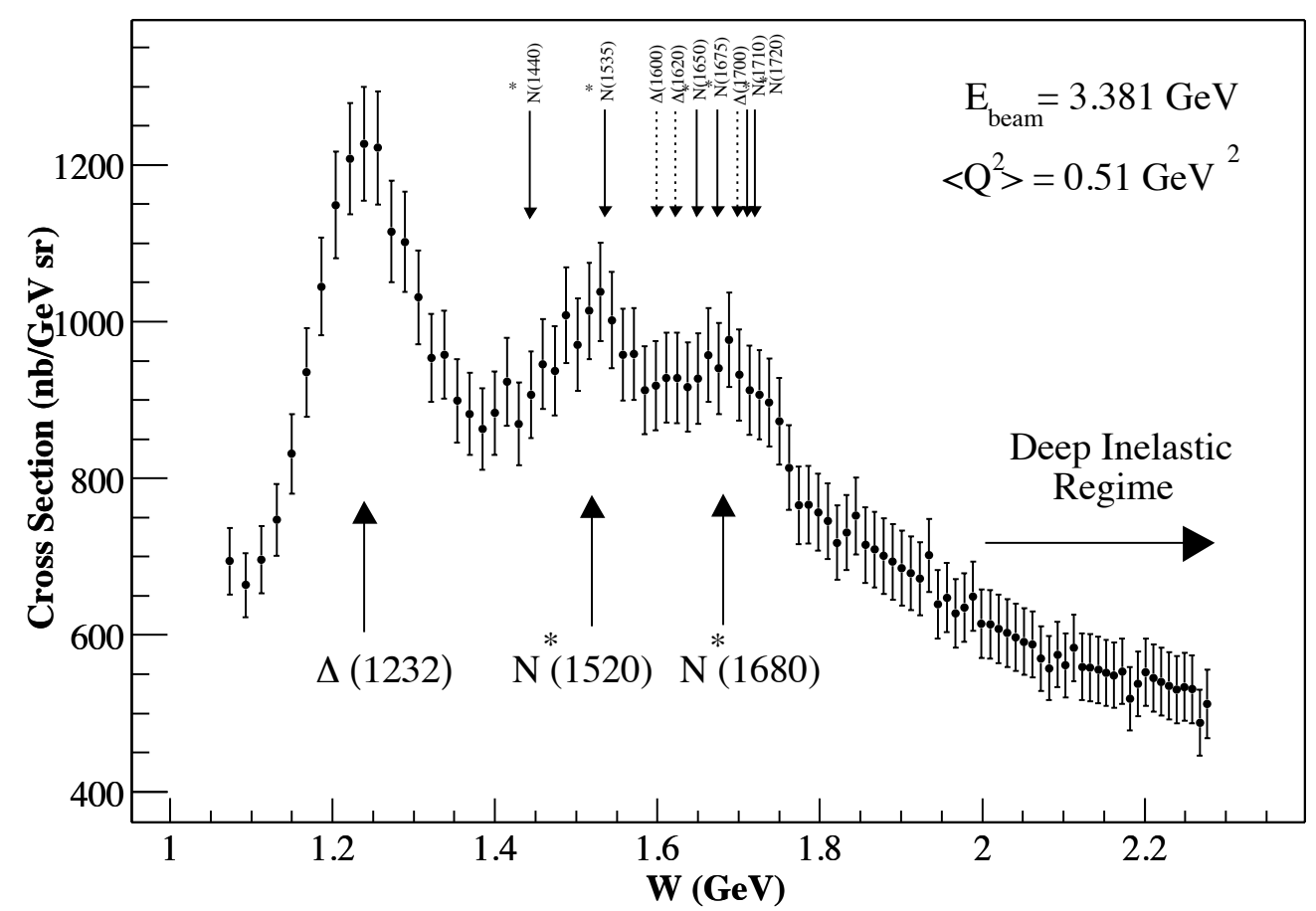

Figure 2.5: Unpolarized ${ }^{3}$ He cross section data in the resonance region from Jefferson Lab experiment E94-010 with some major and minor resonances labeled [20]

$$
\begin{aligned}
& \left.+\frac{2}{3} P_{S^{\prime}}\left[G_{M}^{p 2}\left(Q^{2}\right)-G_{M}^{n 2}\left(Q^{2}\right)\right]\right\} \\
R^{T L^{\prime}}\left(Q^{2}\right)= & \sqrt{2 \tau(1+\tau)}\left\{G_{E}^{n}\left(Q^{2}\right) G_{M}^{n}\left(Q^{2}\right)-\frac{2}{3} P_{D}\left[G_{E}^{p} G_{M}^{p}+2 G_{E}^{n}\left(Q^{2}\right) G_{M}^{n}\left(Q^{2}\right)\right]\right. \\
& +\frac{2}{3} P_{S^{\prime}}\left[G_{E}^{p}\left(Q^{2}\right) G_{M}^{p}\left(Q^{2}\right)-G_{E}^{n}\left(Q^{2}\right) G_{M}^{n}\left(Q^{2}\right)\right] \\
& \left.+2 G_{E}^{p} G_{M}^{n} T\left(Q^{2}\right)\right\}
\end{aligned}
$$

where $P_{D}$ and $P_{S^{\prime}}$ is the effective polarization of the excited states of ${ }^{3} \mathrm{He}$ and $T\left(Q^{2}\right)$ is the Fourier transform of the two-body density matrix. The new elastic cross sections with these substitutions then can be broadened into a Gaussian peak in a similar manner as the unpolarized cross section. 


\subsubsection{Scattering in the resonance and deep inelastic region}

Pions, the lightest hadrons, can be created in hadronic final states from electron scattering on a nucleon at $W \geq 1.072 \mathrm{GeV}=M_{\text {proton }}+m_{\text {pion }}$. Beyond the pion threshhold lies the resonance region and the deep inelastic scattering region. The resonance region is characterized by distinct hadronic final states whose crosssections have a strong $Q^{2}$ dependence. The deep inelastic region is characterized by a slow, logarithmic dependence of the cross-section on $Q^{2}$ which is evidence of incoherent scattering off of individual quarks. The boundary between these two regions is usually defined as $W>2.0 \mathrm{GeV}$.

The resonance region is most acutely marked by the presence of the $\Delta$ resonance at $W=1.232 \mathrm{GeV}$ as shown in Fig. 2.5. However, there are a number of other resonances and a non-resonant background that contribute to the cross section. In inclusive scattering one sees only the sum of all these transitions; therefore, the overall cross section is smoothed out. Also, the use of a nuclear target (like ${ }^{3} \mathrm{He}$ ) smears resonance peaks even more, due to Fermi motion.

The deep inelastic region is characterized by the structure function's weak dependence on $Q^{2}$ for a constant value of $x$. This phenomena is known as Bjorken scaling and can be seen in the $F_{2}^{p}$ data from the NMC experiments displayed in Fig. 2.6 [21]. Scattering off an object that had a finite size would lead to a much stronger $Q^{2}$ dependence; therefore, Bjorken scaling is the result of scattering off of point-like objects, i.e. quarks.

The region where Bjorken scaling is valid can be described by the structure functions in the limit

$$
Q^{2} \rightarrow \infty, \nu \rightarrow \infty, \text { with fixed } x
$$

This is known as the Bjorken limit and in this limit the structure functions become one-dimensional functions of $x$. One interesting feature of this limit is that $R\left(x, Q^{2}\right)$ 
vanishes and $F_{2}$ can be expressed (c.f. Eq. 2.20)

$$
F_{2}(x)=2 x F_{1}(x)
$$

which is known as the Callan-Gross relation.

One can also see from Fig. 2.6 [21] that Bjorken scaling is only an approximation. This is because of the radiation of gluons by the quark before and after the electron scattering process which are analogous to QED radiative effects. These processes cannot be separated from a single vertex process and therefore must be included in the structure functions. Because of this the structure function gains a logarithmic dependence on $Q^{2}$. However, there are clear set of procedures (known as DGLAP evolution) which can be used to evolve the structure functions to different $Q^{2}$ in the deep inelastic regime [7]. 


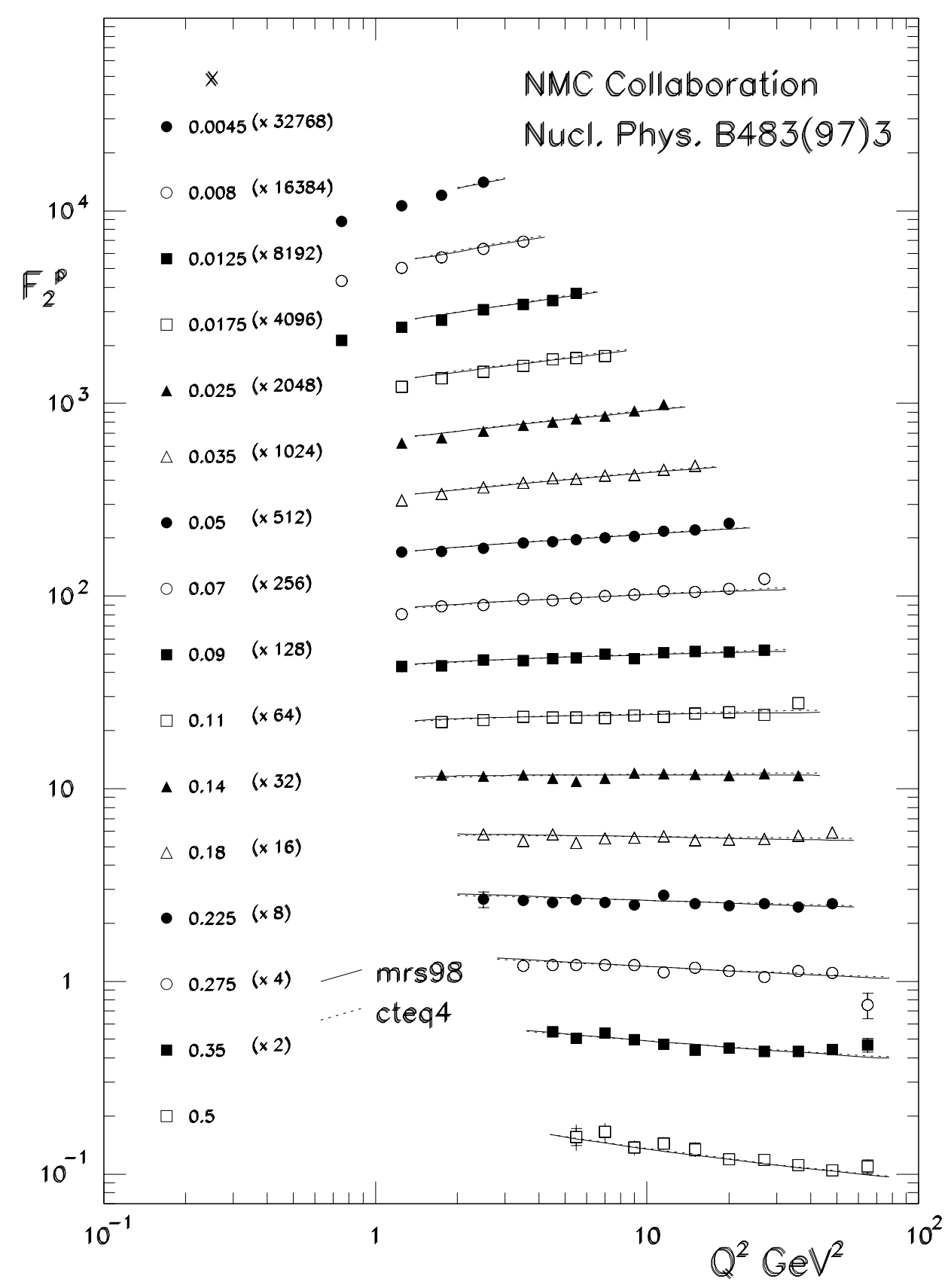

Figure 2.6: $F_{2}^{p}$ from the NMC Collaboration experiments vs. $Q^{2}$ for various values of $x$ [21]. 


\section{CHAPTER 3}

\section{$g_{2}$ and Higher Twist Effects}

\subsection{Why measure $g_{2}$ ?}

Quantum Chromodynamics (QCD), the theory of the strong interaction, has two features which are essential to describing hadrons. The first is that the effects of the strong interaction, though many orders of magnitude stronger than the other fundamental forces, cannot be felt far outside of a hadron. This feature is known as confinement. The second feature of QCD is that when probing the nucleon at high momentum or, equivalently, short distances the coupling constant for the strong interactions goes to zero. This is known as asymptotic freedom.

These properties of QCD complicate direct measurements of the strong interaction. Using low momentum electrons to study nucleons, one sees the quarks in the confinement regime where they are enmeshed in dense gluon fields. Using high momentum electrons to study nucleons, one is in the regime of asymptotic freedom where the gluon fields are so weak that the quarks can be considered non-interacting, the strong force noticeable only through their momentum distributions. To isolate gluon exchanges between quarks, one must study the nucleon in a kinematic regime where the strong coupling constant is large enough for a single gluon exchange to be measured, but not so large that higher order terms begin to dominate.

What makes the structure function $g_{2}$ especially interesting is, because of a relation from Wandzura and Wilczek [22], the asymptotically-free quark part of the structure function can be calculated from the data on $g_{1}$ and subtracted from $g_{2}$. The remaining part of the structure function will be dominated by physics beyond 
the parton model, such as quark-gluon coupling. This means if precise measurements of $g_{1}$ and $g_{2}$ are made one can directly extract the magnitude of gluonic interactions between two quarks. This magnitude is represented theoretically by the so-called higher twist terms in the framework of the operator product expansion, which will be explained below.

There is at present little definite knowledge, from experiment and theory, about the size of the higher twist terms. Prior to E97-103, there were no precise measurements of the structure functions in the right kinematic region to allow an accurate estimate of the higher twist terms. Lattice QCD can be used to calculate sum rule

integrals of $g_{2}$ over $x$, but theoretical predictions of $g_{2}\left(x, Q^{2}\right)$ itself are limited to simple Bag Models. It is the goal of E97-103 to provide measurements of $g_{2}$ in a kinematic region which will allow us to isolate and quantify these higher-twist effects and by doing so improve the quantitative understanding of QCD in the non-perturbative region.

\subsection{Deriving the Wandzura-Wilczek relation}

\subsubsection{A description of the operator product expansion}

The structure functions can be deconstructed directly from the formalism of QCD with the operator product expansion. This method replaces a product of operators with a single local operator. The physical consequences of the original product of operators is revealed by working out the QCD rescaling of this new operator [23]. This approach has the advantages of being model-independent and having coefficients of the expansion that are calculable using perturbation theory.

To illustrate the operator product expansion, one can consider the product of two local operators with the space-time separation $z$ [24]:

$$
\mathcal{O}_{a}(z) \mathcal{O}_{b}(0)
$$


The operators can be considered to be at nearly the same point in the limit $z \rightarrow 0$. If one takes this limit, one can then calculate the product of operators with an expansion of local operators with a series of corresponding coefficients [24] :

$$
\lim _{z \rightarrow 0} \mathcal{O}_{a}(z) \mathcal{O}_{b}(0)=\sum_{k} c_{a b k}(z) \mathcal{O}_{k}(0)
$$

This relation holds as long as $z$ is small compared to the distance scaled probed. This substitution can be used in the computation of matrix elements where the coefficients $c_{a b k}(x)$ will be independent of the matrix element being calculated [24]. Because of asymptotic freedom, the coupling constant of QCD is small at short distances. Therefore it is possible that these coefficient functions can be calculated using perturbation theory, which requires a small coupling constant [24].

The operator expansion also works in momentum space as follows:

$$
\lim _{q \rightarrow \infty} \int d^{4} z e^{i q \cdot z} \mathcal{O}_{a}(z) \mathcal{O}_{b}(0)=\sum_{k} c_{a b k}(q) \mathcal{O}_{k}(0) .
$$

This works provided $q$, the momentum transfer from the probe, is much larger than the characteristic momentum of the external states [24].

\subsubsection{Twist two operators in the operator product expansion}

In QCD, the operator product expansion can be applied to the time-ordered quark electromagnetic currents in the forward Compton scattering amplitude [7]:

$$
T_{\mu \nu}=i \int d^{4} z e^{i q \cdot z}\left\langle N\left|\mathcal{T}\left(j_{\mu}(z) j_{\nu}(0)\right)\right| N\right\rangle
$$

where $\mathcal{T}$ represents the time-ordering operator. This amplitude will be connected to the hadron tensor from Eq. 2.9 by dispersion relations. It is necessary to work with the Compton scattering amplitude so that Wick's theorem can be used.

In this case, the product is expanded into quark and gluon operators with mass dimension $d$ and $\operatorname{spin} n$. These can be written:

$$
\mathcal{O}_{d, n}^{\mu_{1} \ldots \mu_{n}}
$$


where the operator $\mathcal{O}$ is symmetric and traceless under the indices $\mu_{1} \ldots \mu_{n}$. The matrix element of $\mathcal{O}_{d, n}$ in the hadron target is proportional to [24]:

$$
M^{d-n-2} \mathcal{S}\left[p^{\mu_{1}} \ldots p^{\mu_{n}}\right]
$$

for a vector operator, and to

$$
M^{d-n-2} \mathcal{S}\left[s^{\mu_{1}} p^{\mu_{2}} \ldots p^{\mu_{n}}\right]
$$

for an axial operator. $\mathcal{S}$ acts on a tensor to project out the symmetric and traceless component. The power of $M$ is derived from dimensional analysis of the conventional relativistic hadron state [24] where $M$ has the unit of mass.

One can use this information to tell which terms in the expansion are most important and how the other terms are suppressed. This is done by analyzing the $q$ dependence of the terms of the expansion. The coefficient functions $c_{a b k}(q)$ are only functions of $q$. Therefore the free indices of $\mathcal{O}$ must be either $\mu, \nu$ (the indices of the hadron tensor) or must be contracted with $q^{\alpha}$. Every index on $\mathcal{O}$ contracted with $q^{\alpha}$ produces a factor of $p \cdot q$ (or $s \cdot q$ in the case of axial vector) which is of order $Q^{2} / M$. An index $\mu$ or $\nu$ is contracted with the lepton tensor $L_{\mu \nu}$ and results in a factor of $p \cdot k$ or $p \cdot k^{\prime}$, both of which are of order $Q^{2} / M$ [24]. Since the hadronic tensor has a dimension two, the coefficient of $\mathcal{O}$ must have dimension $Q^{2-d}$. The dimensions of the expansion terms can be summarized as follows:

$$
\begin{aligned}
c_{\mu_{1} \ldots \mu_{n}} \mathcal{O}_{d, n}^{\mu_{1} \ldots \mu n} & \rightarrow \frac{q_{\mu_{1}}}{Q} \ldots \frac{q_{\mu_{n}}}{Q} Q^{2-d}\left\langle\mathcal{O}_{d}^{\mu_{1} \ldots \mu_{n}}\right\rangle \\
& \rightarrow \frac{q_{\mu_{1}}}{Q} \ldots \frac{q_{\mu_{n}}}{Q} Q^{2-d} M^{d-n-2} p^{\mu_{1}} \ldots p^{\mu_{n}} \\
& \rightarrow \frac{(p \cdot q)}{Q^{n}} Q^{2-d} M^{d-n-2} p^{\mu_{1}} \ldots p^{\mu_{n}} \\
& \rightarrow\left(\frac{1}{x}\right)^{n}\left(\frac{Q}{M}\right)^{2+n-d}=\left(\frac{1}{x}\right)^{n}\left(\frac{Q}{M}\right)^{2-t}
\end{aligned}
$$

where $t$ is known as the twist of the expansion term and is defined as:

$$
t=d-n
$$




\begin{tabular}{c|ccc} 
& $\psi$ & $G_{\mu \nu}$ & $D^{\mu}$ \\
\hline $\mathrm{d}$ & $3 / 2$ & 2 & 1 \\
$\mathrm{n}$ & $1 / 2$ & 1 & 1 \\
$\mathrm{t}$ & 1 & 1 & 0
\end{tabular}

Table 3.1: The dimension (d), spin ( $n$ ) and twist ( $t)$ of the operators for quarks ( $\psi$ ), gluons $\left(G_{\mu \nu}\right)$ and covariant derivatives $D^{\mu}$.

With this dimensional analysis, one can group the operators into terms by twist using table 3.1. Any gauge invariant operator must contain at least two quark fields or two gluon strength tensors; therefore the lowest possible twist for such an operator is two [24]. For example, a twist two operator has either two quark fields $\psi$ or two gluon operators $G_{\mu \nu}$ and an arbitrary number of covariant derivatives.

Twist two quark operators can be written:

$$
\begin{aligned}
\mathcal{O}_{V, a}^{\mu_{1} \ldots \mu_{n}} & =\frac{1}{2}\left(\frac{i}{2}\right)^{n-1} S\left\{\overline{\psi_{a}} \gamma^{\mu_{1}} D^{\mu_{1}} \ldots D^{\mu_{n}} \psi_{a}\right\} \\
\mathcal{O}_{A, a}^{\mu_{1} \ldots \mu_{n}} & =\frac{1}{2}\left(\frac{i}{2}\right)^{n-1} S\left\{\overline{\psi_{a}} \gamma^{\mu_{1}} D^{\mu_{2}} \ldots D^{\mu_{n}} \gamma_{5} \psi_{a}\right\}
\end{aligned}
$$

where the index $a$ represents the quark flavors $(u, d, s)$. Twist two gluon operators are similar:

$$
\mathcal{O}_{g, V}^{\mu_{1} \ldots \mu_{n}}=-\frac{1}{2}\left(\frac{i}{2}\right)^{n-2} S\left\{G_{a}^{\mu_{1} \alpha} D^{\mu_{2}} \ldots D^{\mu_{n-1}} G_{a \alpha}^{\mu_{n}}\right\}
$$

\subsubsection{QCD coefficients in the operator product expansion}

The next step in the operator product expansion is to determine the coefficient functions of the operators. As stated before, this can be done using perturbative QCD. The generic term in the operator product expansion can be written:

$$
j j \sim c_{q} \mathcal{O}_{q}+c_{g} \mathcal{O}_{g}
$$

where $j j$ is the product of the hadron currents, $c_{q}$ and $c_{g}$ are the coefficients for the quark and gluon operators respectively. One can then take the matrix elements of both sides:

$$
\langle q|j j| q\rangle \sim c_{q}\left\langle q\left|\mathcal{O}_{q}\right| q\right\rangle+c_{g}\left\langle q\left|\mathcal{O}_{g}\right| q\right\rangle
$$


The left hand side of Eq. 3.17 and the first term on the right hand side contain no factors of $g$, the strong coupling constant. The term with the gluon operator, because of the gluon operators, has a factor of $g^{2}$ and is a higher order term. When deriving the Wandzura-Wilczek expression, one is only interested in only the lowest order terms; therefore the second term will be dropped.

A detailed description of deriving the left hand side of Eq. 3.17 can be found in Ref. [24]. The spin-dependent part of the left hand side is revealed to be:

$$
\mathcal{M}^{[\mu \nu]}=\sum_{n=0,2,4}^{\infty} 2 \frac{2^{n+1} q^{\mu_{2}} \ldots q^{\mu_{n}}}{\left(-q^{2}\right)^{n+1}} i h \epsilon^{\mu \nu \alpha \mu_{1}} q_{\alpha} p_{\mu_{1}} \ldots p_{\mu_{n}} .
$$

Notice that the matrix element only contains even spin terms. This is because even spin axial current vectors are even under charge conjugation. The odd spin are odd. Since electro-production is even under charge conjugation, the odd spin terms are dropped.

To compute the spin-dependent part of the right hand side of Eq. 3.17 one needs to evaluate the hadronic matrix elements of the operator in Eq. 3.14. Unfortunately, the matrix elements of the operators for a nucleon are not known. However, the matrix elements can be written :

$$
\left\langle p, s\left|\mathcal{O}_{A}^{\mu_{1} \ldots \mu_{n}}\right| p, s\right\rangle=a_{n} \mathcal{S}\left[s^{\mu_{1}} \ldots p^{\mu_{n}}\right]
$$

where $a_{n}$ is a scaler factor and $\mathcal{S}$ is defined as:

$$
\mathcal{S}\left[s^{\mu_{1}} \ldots p^{\mu_{n}}\right]=s^{\mu_{1}} \ldots p^{\mu_{n}}+\mathcal{R}^{\mu_{1} \ldots \mu_{n}}
$$

and where :

$$
\mathcal{R}^{\mu_{1} \ldots \mu_{n}}=\frac{n}{n+1} s^{\mu_{1}} p^{\mu_{2}} \ldots p^{\mu_{n}}+\frac{1}{n+1} p^{\mu_{1}} s^{\mu_{2}} \ldots p^{\mu_{n}}+\ldots+\frac{1}{n+1} p^{\mu_{1}} p^{\mu_{2}} \ldots s^{\mu_{n}}
$$

It is an important point that the tensor $\mathcal{R}^{\mu_{1} \ldots \mu_{n}}$ has no completely symmetric part and is therefore spin $n-1$, instead of spin $n$. Thus the tensor contribution or $\mathcal{R}$ is 
twist three even though it came from a twist two matrix element. This is an essential point in deriving the Wandzura-Wilczek relation.

If one equates Eq. 3.18 and Eq. 3.19 one arrives at:

$$
\sum_{n=0,2,4}^{\infty} 2 \frac{2^{n+1} q^{\mu_{2}} \ldots q^{\mu_{n}}}{\left(-q^{2}\right)^{n+1}} i h \epsilon^{\mu \nu \alpha \mu_{1}} q_{\alpha} p_{\mu_{1} \ldots \mu_{n}}=\sum_{n=0,2,4}^{\infty} c_{n} a_{n} \mathcal{S}\left[s^{\mu_{1}} \ldots p^{\mu_{n}}\right]
$$

One can then calculate the $c_{q}$ coefficient, with the knowledge that $a_{n}$ is still not known. Thus the antisymmetric part of the anti-symmetric part of the Compton scattering matrix $T^{\mu \nu}$ is:

$$
T^{[\mu \nu]}=\sum_{n=0,2,4}^{\infty} 2 C_{n} i \epsilon^{\mu \nu \alpha \mu_{1}} q_{\alpha} \frac{2^{n+1} q^{\mu_{2}} \ldots q^{\mu_{n}}}{\left(-q^{2}\right)^{n+1}} a_{n} \mathcal{S}\left[s^{\mu_{1}} \ldots p^{\mu_{n}}\right]
$$

where $C_{n}$ is a term to take into account the charge of the quark flavor (which previously has been ignored).

\subsubsection{Extracting relations for $g_{1}$ and $g_{2}$}

If one ignores the $R$ tensor contribution to Eq. 3.23 and combine up terms with matching indices then one has:

$$
T^{[\mu \nu]}=\sum_{n=0,2,4}^{\infty}-\frac{4}{q^{2}} C_{n} i \epsilon^{\mu \nu \alpha \mu_{1}} q_{\alpha} s_{\mu_{1}} a_{n}\left(\frac{1}{x}\right)^{n} .
$$

One can see this similar to the $\tilde{g}_{1}$ term in the scattering amplitude:

$$
T^{[\mu \nu]}=\sum_{n=0,2,4}^{\infty} i \epsilon^{\mu \nu \alpha \lambda} s_{\lambda} \frac{\tilde{g}_{1}}{p \cdot q}
$$

where $\tilde{g}_{1}$ is the spin structure function of Compton scattering. One then equates the previous equations to get:

$$
\begin{aligned}
& \tilde{g}_{1}=\sum_{n=0,2,4}^{\infty}-\frac{4 p \cdot q}{q^{2}} C_{n} a_{n}\left(\frac{1}{x}\right)^{n} \\
& \tilde{g}_{1}=\sum_{n=0,2,4}^{\infty} 2 C_{n} a_{n}\left(\frac{1}{x}\right)^{n+1} .
\end{aligned}
$$

Already this is a remarkable result. The operator product expansion has allowed the spin structure function $\tilde{g}_{1}$ in terms of a power series of $1 / x$. 
However, it is necessary to relate $\tilde{g}_{1}$ to the physical region. One can do this by calculating the contour integral [24]:

$$
2 C_{n} a_{n}=\frac{1}{2 \pi i} \oint_{C} \tilde{g}_{1}(\omega) \frac{d \omega}{\omega^{n+2}}
$$

and using the optical theorem:

$$
\tilde{g}_{1}(\omega+i \epsilon)-\tilde{g}_{1}(\omega-i \epsilon)=4 \pi i g_{1}(\omega)
$$

where $\omega=1 / x$ and $g_{1}(\omega)$ is the measurable spin structure function. The result of this integral is:

$$
2 C_{n} a_{n}=2\left[1-(-1)^{n+1}\right] \int_{1}^{\infty} g_{1}(\omega) \frac{d \omega}{\omega^{n+2}} .
$$

The translation of this equation into $x$ instead of $\omega$ is:

$$
2 \int_{0}^{1} d x x^{n} g_{1}(x)=C_{n} a_{n}, n \text { even. }
$$

This is a calculation of the twist two moment for $g_{1}$.

Up to this point, the $\mathcal{R}$ tensor in Eq. 3.21 has been ignored. If the $\mathcal{R}$ tensor is inserted into Eq. 3.23 then one gets:

$$
T^{[\mu \nu]}=\sum_{n=0,2,4}^{\infty}-\frac{n}{n+1}\left[s^{\mu_{1}} \frac{2^{n+1}(p \cdot q)^{n}}{\left(-q^{2}\right)^{n+1}}-p^{\mu_{1}} \frac{2^{n+1}(p \cdot q)^{n-2} q \cdot s}{\left(-q^{2}\right)^{n+1}}\right] 2 C_{n} a_{n} i \epsilon^{\mu \nu \alpha \mu_{1}} q_{\alpha} .
$$

This kinematic structure is the same as the Compton scattering spin structure function $\tilde{g}_{2}$ which can be written:

$$
\tilde{g}_{2}(\omega)=\sum_{n=0,2,4}^{\infty} 2 C_{n} a_{n}\left[\frac{1}{n+1}-1\right] \omega^{n+1} .
$$

Substituting Eq. 3.26 into the above equation one gets the relation:

$$
\tilde{g}_{2}(\omega)=\tilde{g}_{1}(\omega)+\int_{0}^{\omega} \tilde{g}_{1}\left(\omega^{\prime}\right) \frac{d \omega^{\prime}}{\omega^{\prime}} .
$$

This can be translated into the physical regime using the optical theorem:

$$
g_{2}(x)=g_{2}^{\mathrm{WW}}(x)=-g_{1}(x)+\int_{x}^{1} g_{1}\left(x^{\prime}\right) \frac{d x^{\prime}}{x^{\prime}} .
$$




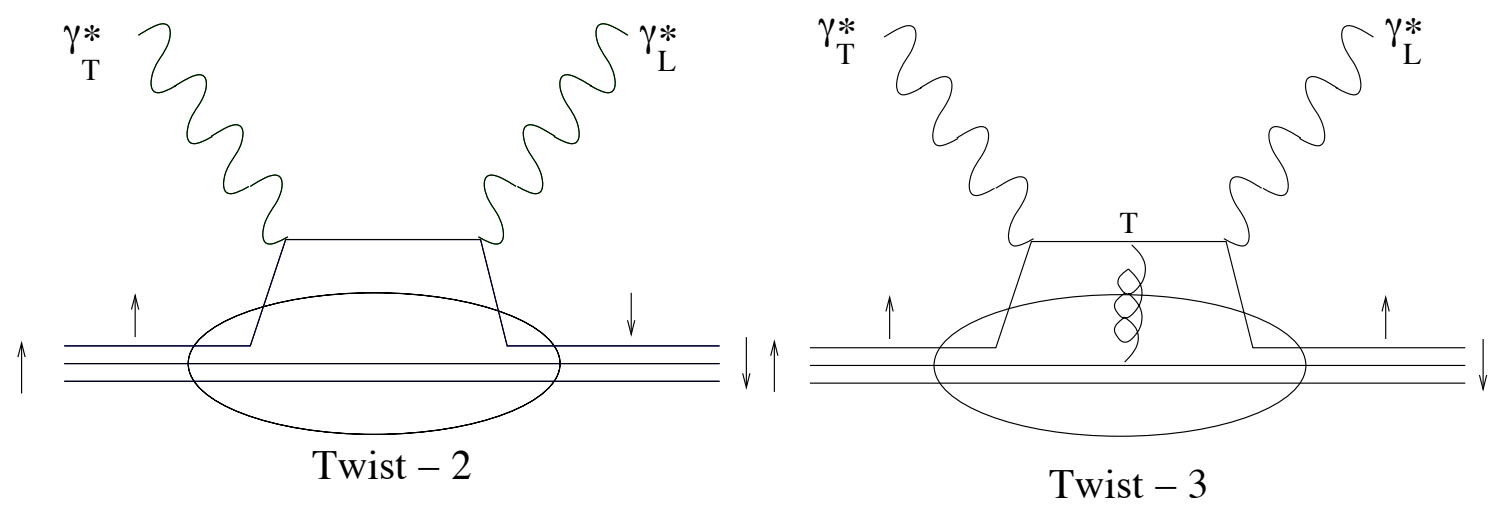

Figure 3.1: Compton scattering diagrams for twist two and twist three operators. The arrows label the spin of the quarks and nucleon.

This is the Wandzura-Wilczek relation [22]. It is derived by using the operator product expansion and cutting off terms with twist higher than two. It shows that the contribution to $g_{2}$ from the twist two operator is purely a function of $g_{1}$. This part that can written in terms of $g_{1}$ is usually written $g_{2}^{\mathrm{WW}}$.

\subsubsection{Twist three operators}

The structure functions $g_{1}\left(x, Q^{2}\right)$ and $g_{2}\left(x, Q^{2}\right)$ have been calculated from their equivalents in Compton scattering and using the optical theorem. An interpretation of $g_{2}\left(x, Q^{2}\right)$ can be made by looking at its Compton scattering counterpart. $g_{2}\left(x, Q^{2}\right)$ is the imaginary part of the process [25]:

$$
\gamma^{\star}(+1)+N(1 / 2) \rightarrow \gamma^{\star}(0)+N(-1 / 2)
$$

where $\gamma^{\star}$ and $N$ represent the virtual photon and nucleon, respectively, and the numbers in parenthesis are their helicities. This process is illustrated by the right diagram in Fig. 3.1.

Because of the vector coupling, the helicity of massless quarks cannot be flipped in perturbative processes. There are two processes which can perform the helicity exchange : first, single quark scattering in which the quark absorbs the helicity through its transverse momentum and, second, through quark scattering where the 
quark also absorbs a transversely polarized gluon from another quark. Both of these processes from twist three operators.

As seen from the dimensional analysis of Eq. 3.8 terms with twist higher than two are suppressed by increasing powers of $M / Q$. This is not the case in the confinement region where higher twist terms dominate. Nevertheless, in the deep inelastic scattering region, where measurements of E97-103 takes place, the twist three terms can be expected to dominate the higher twist terms.

The twist three with two quark fields and a gluon field can be written [26]:

$$
\begin{aligned}
R_{l}^{\sigma \mu_{1} \ldots \mu_{n}} & =\frac{i^{n-3}}{4} g \mathcal{S}\left[\bar{\psi}(0) D^{\mu_{1}} \ldots D^{\mu_{l-1}} G^{\sigma \mu_{1}} D^{\mu_{l+1}} \ldots D^{\mu_{n-1}} \gamma^{\mu_{n}} \gamma^{5} \psi(0)\right] \\
S_{l}^{\sigma \mu_{1} \ldots \mu_{n}} & =\frac{i^{n-2}}{4} g \mathcal{S}\left[\bar{\psi}(0) D^{\mu_{1}} \ldots D^{\mu_{l-1}} \tilde{G}^{\sigma \mu_{l}} D^{\mu_{l+1}} \ldots D^{\mu_{n-1}} \gamma^{\mu_{n}} \psi(0)\right] .
\end{aligned}
$$

There is also an explicit quark mass-dependent operator that is twist three:

$$
O_{m}^{\sigma \mu_{1} \mu_{2} \ldots \mu_{n}}=\frac{i^{n+1}}{4} \mathcal{S}\left[\bar{\psi}(0) m_{q}\left[\gamma^{\sigma}, \gamma^{\mu_{1}}\right] \gamma^{5} D^{\mu_{2}} \ldots D^{\mu_{n}} \psi(0)\right]
$$

where $m_{q}$ is the quark mass.

In $g_{1}$, the twist three terms are suppressed by an additional $M / Q$ term compared to the twist two term [27]. However, in $g_{2}$ the twist three enter at the same order of $M / Q$ as the twist two term. To leading order in $M / Q$, the moments of the structure functions up to twist three can be written [26]:

$$
\begin{aligned}
\int_{0}^{1} x^{n} g_{1}\left(x, Q^{2}\right) d x= & \frac{1}{2} \sum_{i} a_{i}^{n}\left(\mu^{2}\right) C_{2, i}^{n}\left(Q^{2}, \mu^{2}\right), n=0,2,4, \ldots \\
\int_{0}^{1} x^{n} g_{2}\left(x, Q^{2}\right) d x= & \frac{n}{2(n+1)}\left[\sum_{i} a_{i}^{n}\left(\mu^{2}\right) C_{2, i}^{n}\left(Q^{2}, \mu^{2}\right)\right. \\
& \left.-\sum_{i} d_{i}^{n}\left(\mu^{2}\right) C_{3, i}^{n}\left(Q^{2}, \mu^{2}\right)\right], n=2,4, \ldots
\end{aligned}
$$

where the $a_{i}^{n}\left(\mu^{2}\right)$ is the twist two matrix element coefficient with its dependence on renormalization scale $\left(\mu^{2}\right)$ made explicit, $d_{i}^{n}\left(\mu^{2}\right)$ is the twist three matrix element coefficient and $C_{2, i}^{n}\left(Q^{2}, \mu^{2}\right)$ and $C_{3, i}^{n}\left(Q^{2}, \mu^{2}\right)$ are the operator product expansion coefficients for twist two and twist three operators. 
Looking at these moments of $g_{1}$ and $g_{2}$, the value of measuring $g_{2}$ again becomes evident. If the only terms in the operator expansion written to leading order in $M / Q$, one can write $g_{2}$ as:

$$
g_{2}\left(x, Q^{2}\right)=g_{2}^{\mathrm{WW}}\left(x, Q^{2}\right)+\overline{g_{2}}\left(x, Q^{2}\right)
$$

where $g_{2}^{\mathrm{WW}}\left(x, Q^{2}\right)$ is the contribution to $g_{2}$ from twist two terms in the operator expansion and $\bar{g}_{2}\left(x, Q^{2}\right)$ represents the contribution from twist three operators. Therefore, if one knows $g_{1}\left(x, Q^{2}\right)$, accurately and one believes the terms beyond twist two are suppressed sufficiently, then $g_{2}^{\mathrm{WW}}\left(x, Q^{2}\right)$ can be calculated. The twist two part of $g_{2}$ can then be subtracted from $g_{2}\left(x, Q^{2}\right)$ and the term $\overline{g_{2}}\left(x, Q^{2}\right)$ can be isolated. The function $\overline{g_{2}}\left(x, Q^{2}\right)$ is expected to be dominated, even when the full expansion is included, by the twist three gluon operators and there is no reason it should be small [26].

\section{$\underline{3.3 \quad \text { Models of } g_{2}}$}

\subsubsection{The parton model}

The parton model begins with the assumption hadrons are made up of free partons. The hadron tensor can then be formed by combinations of the distributions functions of these partons. This assumption has considerable validity in the deep inelastic scattering region because of the asymptotic freedom of the quarks. It is useful because of its straight-forward interpretation for the structure functions, $F_{1}$ and $g_{1}$. However, complex modifications to these assumptions are necessary to get a physical description of $g_{2}$.

To lowest order in QCD, virtual photons only scatter off of quarks because gluons have no electric charge. The interaction is pictured in Fig. 3.2. Within the framework of inclusive electron scattering, this makes the hadron tensor $W_{\mu \nu}$ very similar to the lepton tensor $l_{\mu \nu}$. An additional subtlety of the parton scattering 


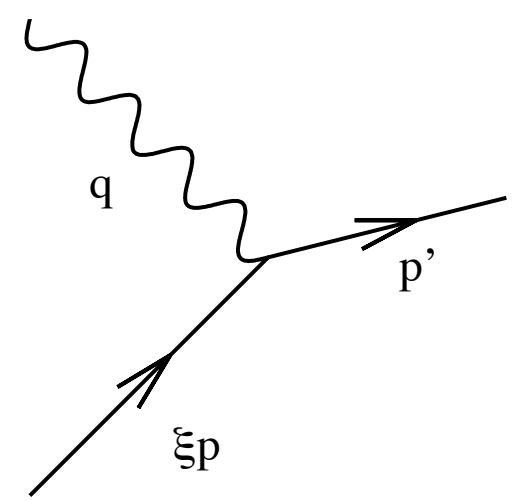

Figure 3.2: Feynman diagram of a parton with momentum fraction $\xi$ absorbing a virtual photon.

case is that the parton's momentum is only a fraction, $\xi$ of the observed hadron momentum. By making the substitutions $k \rightarrow \xi p, k^{\prime} \rightarrow p^{\prime}$ and $q \rightarrow-q$, multiplying by the square of quark electron charge $\mathcal{Q}$ and assuming massless partons one can transform the lepton tensor $l_{\mu \nu}$ into the hadron tensor [24]:

$$
\begin{aligned}
W_{\mu \nu}= & \frac{1}{4 \pi} \int \frac{d^{3} p^{\prime}}{(2 \pi)^{3} 2 E_{p^{\prime}}} \frac{1}{\xi}(2 \pi)^{4} \delta^{4}\left(\xi p+q-p^{\prime}\right) \\
& \times 2\left[\xi p^{\mu} p^{\prime \nu}+\xi p^{\nu} p^{\mu}-g^{\mu \nu} \xi p \cdot p^{\prime}+i \epsilon^{\mu \nu \alpha \beta} q_{\alpha} s_{\beta}\right] .
\end{aligned}
$$

This integral can be performed using the identity:

$$
\int \frac{d^{3} p^{\prime}}{(2 \pi)^{3} 2 E_{p^{\prime}}}=\int \frac{d^{4} p^{\prime}}{(2 \pi)^{4}}(2 \pi) \delta\left(\left(\xi p+q-p^{\prime}\right)^{2}\right)=\int \frac{d^{4} p^{\prime}}{(2 \pi)^{4}} \frac{(2 \pi)}{2 p \cdot q} \delta\left(\xi+\frac{q^{2}}{2 p \cdot q}\right)
$$

Since the partons are massless one can write $s_{\beta}=h \xi p_{\beta}$ where $h$ is the helicity of the parton. Then one can write the hadron tensor as:

$$
\begin{aligned}
W_{\mu \nu} & =\frac{\mathcal{Q}^{2}}{2 \xi p \cdot q}\left[\xi p^{\mu} p^{\prime \nu}+\xi p^{\prime} p^{\mu}-g^{\mu \nu} \xi p \cdot p^{\prime}+i h \xi e^{\mu \nu \alpha \beta} q_{\alpha} p_{\beta}\right] \delta(\xi-x) \\
& =\frac{\mathcal{Q}^{2}}{2 \xi p \cdot q}\left[2 \xi^{2} p^{\mu} p^{\nu}-g^{\mu \nu} \xi p \cdot q+i h \xi \epsilon^{\mu \nu \alpha \beta} q_{a l p h a} p_{\beta}\right] \delta(\xi-x)
\end{aligned}
$$

Because of Eq. 3.44, $p^{\prime}$ can be replaced with $\xi p+q$ and the $q^{\mu}$ and $q^{\nu}$ terms can be dropped. The total target spin $s_{h}$ can be replaced with $\mathcal{H} p$, where $\mathcal{H}$ is the helicity of the target and $p$ is the total target momentum. Using this substitution 
and the kinematic structure of Eq. 2.12 and Eq. 2.13 one can calculate the structure functions:

$$
F_{1}=\frac{\mathcal{Q}^{2}}{2} \delta(\xi-x), F_{2}=\mathcal{Q}^{2} \xi \delta(\xi-x), g_{1}=\frac{\mathcal{Q}^{2}}{2} h \mathcal{H} \delta(\xi-x), g_{2}=0 .
$$

However, this is only the structure function for a single quark with a known helicity. Nucleons will contain a distribution of quarks and anti-quarks with different helicities. The total structure function is obtained by sums of probabilities to find a quark (or anti-quark) with that helicity as shown here:

$$
\begin{aligned}
& F_{1}\left(x, Q^{2}\right)=\sum_{i} \frac{\mathcal{Q}_{i}^{2}}{2}\left(q_{+}(x)+q_{-}(x)+\bar{q}_{+}(x)+\bar{q}_{-}(x)\right) \\
& F_{2}\left(x, Q^{2}\right)=\sum_{i} \mathcal{Q}_{i}^{2} x\left(q_{+}(x)+q_{-}(x)+\bar{q}_{+}(x)+\bar{q}_{-}(x)\right) \\
& g_{1}\left(x, Q^{2}\right)=\sum_{i} \frac{\mathcal{Q}_{i}^{2}}{2}\left(q_{+}(x)-q_{-}(x)+\bar{q}_{+}(x)-\bar{q}_{-}(x)\right) \\
& g_{2}\left(x, Q^{2}\right)=0
\end{aligned}
$$

where $q_{+}$is the probability of a quark having the helicity of the nucleon, $q_{-}$is the probability of a quark having the opposite helicity of the nucleon and $\bar{q}_{+}$and $\bar{q}_{-}$are the anti-quark probabilities with same and opposite helicity respectively.

From this set of equations one can already seen some physical behavior of the structure functions. First, the structure functions depend only on $x$ and not on $Q^{2}$. This result reflects the Bjorken scaling present in experimental data at high $Q^{2}$. Secondly, the Callan-Gross relation, seen in Eq. 2.50, is satisfied.

Also, one can see clear interpretations of some of the structure functions. $F_{1}$ is the probability of finding a quark with momentum fraction $x . g_{1}$ is the difference in probabilities between quarks with momentum $x$ with spin parallel to the nucleon spin and quarks with momentum $x$ with spin anti-parallel to the nucleon spin.

Whatever positive qualities the parton model has, it completely fails to describe $g_{2}$. This is because the parton model assumes all the quarks have collinear 
momentum and are non-interacting. As has been explained earlier, $g_{2}$ is the result of transverse momentum and quark-gluon interactions; therefore, it is not surprising to find $g_{2}\left(x, Q^{2}\right)=0$.

One way to extend the parton distribution to describe $g_{2}$ is to include transverse momentum, $\vec{p}_{\perp}$ in the model. Then one can write the $g_{2}$ from above as [7]:

$$
g_{2}\left(x, Q^{2}\right)=\sum_{i} \frac{\mathcal{Q}^{2}}{2}\left(\frac{m_{q}}{x M}-1\right)\left(q_{+}(x)-q_{-}(x)+\bar{q}_{+}(x)-\bar{q}_{-}(x)\right)
$$

where $m_{q}$ is the quark mass and $M$ is the nucleon mass. Clearly, if $m_{q}=x M$, an initial assumption in the parton model, then $g_{2}\left(x, Q^{2}\right)=0$ again. However, if $m_{q} \neq x M$ then the quark is said to be off-mass-shell and $g_{2}(x) \neq 0$. Therefore, $g_{2}$ measures the degree the quarks in the nucleon are off-mass-shell.

Another approach is to define new parton distributions corresponding to the transverse momentum [28]. In terms of these newly distributions one can write $g_{2}$ as:

$$
g_{2}\left(x, Q^{2}\right)=\sum_{i} \frac{\mathcal{Q}^{2}}{2}\left(q_{T+}(x)-q_{T-}(x)+\bar{q}_{T+}(x)-\bar{q}_{T-}\right)
$$

where $q_{T}$ is the transverse parton distribution.

However, even this approach reveals the difficulty of describing $g_{2}$ with a parton model. $q_{T}$ does not evolve autonomously under scale transformation; therefore, one has to separate the transverse parton distribution into two two-parameter parton distributions [25]:

$$
\Delta q_{T}(x)=\frac{2}{x} \int_{-1}^{1} d y\left(K_{1}(x, y)+K_{2}(x, y)\right)
$$

where $K_{1}(x, y)$ and $K_{2}(x, y)$ are defined in such a way that the scale transformation is autonomous. A summary of the various approaches to the parton distributions can be found in Ref. [25]. In any case, most of the work on these parton distributions goes into predicting the matrix elements $d_{n}$ and not $g_{2}\left(x, Q^{2}\right)$ for particular $x$ and $Q^{2}$. 


\subsubsection{Bag Models of $g_{2}$}

Bag models try to improve on the parton model by building a framework that incorporates the confinement phenomenology of QCD. In general, a bag model does this by separating the nucleon into two spaces : an interior, where the quarks have small masses and weakly interact, and an exterior, in which the quarks are not allowed to propagate and have a different vacuum energy [7]. Since confinement interactions are built in, these models potentially could describe the quark-gluon interaction part of $g_{2}$ in a more straight-forward manner.

While there are several types of bag models, the one given here will be the modified center-of-mass bag model which has been used to calculate $g_{2}\left(x, Q^{2}\right)$ [29]. This model make a series of assumptions. First, the virtual photon interacts with one quark at a time and the other two are spectators. Secondly, the nucleon is assumed to be in a Fock state with three valence quarks. Finally, the effect of quark confinement is described in terms of a bound state quark spatial wave function.

Based on these assumptions the hadron current can be written:

$$
\begin{aligned}
\int d^{4} y e^{i q \cdot y}\left\langle p^{\prime}\left|J_{\mu}(y)\right| p\right\rangle & =(2 \pi)^{4} \delta^{4}\left(p+q-p^{\prime}\right)\left\langle p^{\prime}\left|J_{\mu}(0)\right| p\right\rangle \\
& =(2 \pi) \delta^{4}\left(p+q-p^{\prime}\right) \\
& \times \sum_{1 \rightarrow 2,3} \int\left(\prod_{i=1}^{3} d^{3} \mathbf{r}_{\mathbf{i}}\right) e^{i \mathbf{q} \cdot \mathbf{r}_{\mathbf{i}}} \bar{q}_{p^{\prime}, \alpha^{\prime}}\left(\mathbf{r}_{1}, \mathbf{r}_{2}, \mathbf{r}_{3}\right)\left[\hat{e}_{q} \gamma_{\mu}\right]_{1} q_{p, \alpha}\left(\mathbf{r}_{1}, \mathbf{r}_{2}, \mathbf{r}_{3}\right) .
\end{aligned}
$$

where $\mathbf{r}_{i}$ is the position of the quark with respect to the center of mass, $\hat{e}_{q}$ is the charge operator of the struck quark and $q_{p, \alpha}$ is the nucleon wave function. The subscript 1 of the operator $\left[\hat{e}_{q} \gamma_{\mu}\right]_{1}$ denotes that the operator appears in the terms concerning the quark that is struck by the virtual photon and not the two other quark terms. The nucleon wave can be written in further detail:

$$
q_{p, \alpha}\left(\mathbf{r}_{1}, \mathbf{r}_{2}, \mathbf{r}_{3}\right)=\prod_{i=1}^{3} q_{p, m}\left(\mathbf{r}_{i}\right) \alpha_{N}
$$

where $\alpha_{N}$ is the wave function of the nucleon and $q_{p, m}$ is the wave function of a 
bound quark.

Using Eq. 3.55, one can then write the hadronic tensor as [29]:

$$
\begin{aligned}
W_{\mu \nu}(P, q, S)= & \sum_{1 \rightarrow 2,3} \sum_{\alpha_{1}, m_{1}} b_{\alpha_{1}, m_{1}}(1 ; 23) \frac{M}{(2 \pi)^{6} R_{1}^{3}} \\
& \times \int \prod_{i=1}^{3} \frac{d^{3} \mathbf{k}_{i}}{2 k_{i}} \delta^{4}\left(q+P-\sum_{i} k_{i}\right) I_{m_{1} \mu \nu}\left(\mathbf{k}_{1}-\mathbf{q}\right) I_{m_{2}}\left(\mathbf{k}_{2}\right) I_{m_{3}}\left(\mathbf{k}_{3}\right)
\end{aligned}
$$

where $b_{\alpha_{1}, m_{1}}(1 ; 23)$ is the matrix element of $\hat{e}_{q}^{2}, \mathbf{k}_{i}$ and $m_{i}$ are the three-momentum and spin projections of the $i$ th quark and $R_{i}$ are the parameters which determine the radius of the quark distribution. The integral :

$$
I_{m_{1}, \mu \nu}\left(\mathbf{k}_{1}-\mathbf{q}\right) \equiv \int d^{3} \mathbf{r}_{1}^{\prime} \int d^{3} \mathbf{r}_{1} e^{i\left(\mathbf{k}_{1}-\mathbf{q}\right) \cdot\left(\mathbf{r}_{1}-\mathbf{r}_{1}^{\prime}\right)} \bar{q}_{m_{1}}\left(\mathbf{r}_{1}\right) \gamma_{\mu} \not \gamma_{1} \gamma_{\nu} q_{m_{1}}\left(\mathbf{r}_{\mathbf{1}}^{\prime}\right)
$$

denotes the contribution from the struck quark where:

$$
I_{m_{j}}\left(\mathbf{k}_{j}\right) \equiv \int d^{3} \mathbf{r}_{j} \int d^{3} \mathbf{r}_{\mathbf{j}}^{\prime} e^{i\left(\mathbf{k}_{j}\right) \cdot\left(\mathbf{r}_{1}-\mathbf{r}_{1}^{\prime}\right)} \bar{q}_{m_{j}}\left(\mathbf{r}_{j}\right) \gamma_{\mu} \not k_{j} \gamma_{0} q_{m_{j}}\left(\mathbf{r}_{\mathbf{j}}^{\prime}\right)(j=2,3)
$$

denotes the contribution from the spectator quarks. These quark wave functions can be calculated using the cavity solution to the MIT bag model or other bound quark wave function in relativistic quark model. A summary of these approaches can be found in Ref. [29].

Two calculations have been made at $Q^{2}=1.0 \mathrm{GeV}^{2}$ using bag models for $g_{2}^{n}(x)$ one by X. Song [30] and one by M. Stratmann [31]. These are shown in Fig. 3.4.

\section{$\underline{3.4 \quad \text { Experimental data on } g_{2}}$}

While polarized spin structure function measurements in the deep inelastic regime have been on-going since the late 1980's, the data on $g_{2}$ for both the proton and the neutron are sparse. This is because most of these measurements put an emphasis on $g_{1}\left(x, Q^{2}\right)$. The E155X experiment at the Stanford Linear Acclerator Facility (SLAC) is the only previous dedicated $g_{2}\left(x, Q^{2}\right)$ measurement in the deep 


\begin{tabular}{lcccc}
\hline Experiment & $x$ range & $Q^{2}$ range & $\begin{array}{c}\text { number of } \\
g_{1}^{p} \text { points }\end{array}$ & Ref. \\
\hline E143 & $0.027-0.749$ & $1.17-9.52$ & 28 & {$[32]$} \\
HERMES & $0.028-0.660$ & $1.13-7.46$ & 39 & {$[33]$} \\
E155 & $0.015-0.750$ & $1.22-34.72$ & 24 & {$[34]$} \\
SMC & $0.005-0.480$ & $1.30-58.0$ & 12 & {$[35]$} \\
EMC & $0.015-0.466$ & $3.50-29.5$ & 10 & {$[36]$} \\
\hline
\end{tabular}

Table 3.2: Description of the DIS world data set on $g_{1}^{p}$.

\begin{tabular}{lccccc}
\hline Experiment & target & $x$ range & $Q^{2}$ range & $\begin{array}{c}\text { number of } \\
g_{1}^{n} \text { points }\end{array}$ & Ref. \\
\hline E143 & d & $0.027-0.729$ & $1.17-9.52$ & 28 & {$[37]$} \\
E155 & $\mathrm{d}$ & $0.015-0.750$ & $1.22-34.79$ & 24 & {$[34]$} \\
SMC & $\mathrm{d}$ & $0.005-0.479$ & $1.30-54.8$ & 12 & {$[35]$} \\
E142 & ${ }^{3} \mathrm{He}$ & $0.035-0.466$ & $1.10-5.50$ & 8 & {$[38]$} \\
HERMES & ${ }^{3} \mathrm{He}$ & $0.033-0.464$ & $1.22-5.25$ & 9 & {$[39]$} \\
E154 & ${ }^{3} \mathrm{He}$ & $0.017-0.564$ & $1.20-15.0$ & 17 & {$[40]$} \\
E99-117 & ${ }^{3} \mathrm{He}$ & $0.327-0.601$ & $2.709-4.833$ & 3 & {$[41]$} \\
\hline
\end{tabular}

Table 3.3: Description of the previous DIS world data set on $g_{1}^{n}$.

inelastic scattering region. The Jefferson Lab experiment E99-117, while focused on measuring $A_{1}^{n}$, also performed a precise measurement of $g_{2}^{n}$.

The data taken on $g_{1}$ and $g_{2}$ are summarized in the Tables 3.2, 3.3, 3.4 and 3.5. Also in Fig. 3.3 and Fig. 3.4 the best data on $g_{2}^{p}$ and $g_{2}^{n}$ are presented. The rest of the world data for $g_{2}$ for both proton and the neutron is much less precise and is not included in the tables and in the plots.

Even though higher twist effects should be present in both the proton and the neutron, E97-103 made the choice of searching for higher twist effects in $g_{2}^{n}$ over $g_{2}^{p}$ for two reasons. First, $g_{1}^{p}$ is 3-5 times larger than $g_{1}^{n}$ in the kinematic region of interest, even though $g_{2}^{p}$ and $g_{2}^{n}$ are roughly the same size. Since measurements of $g_{2}$ always have a $g_{1}$ background contribution, the smaller $g_{1}$ is, the cleaner the measurement of $g_{2}$. Secondly, there are certain practical advantages of a polarized ${ }^{3} \mathrm{He}$ target for measuring $g_{2}$ rather than a polarized proton target. In any case, one can see that more measurements of $g_{2}$ are warranted. 


\begin{tabular}{lcccc}
\hline Experiment & $x$ range & $Q^{2}$ range & $\begin{array}{c}\text { number of } \\
g_{2}^{p} \text { points }\end{array}$ & Ref. \\
\hline E155X & $0.021-0.780$ & $0.8-0.780$ & 10 & {$[34]$} \\
\hline
\end{tabular}

Table 3.4: Description of DIS world data set on $g_{2}^{p}$.

\begin{tabular}{lccccc}
\hline Experiment & target & $x$ range & $Q^{2}$ range & $\begin{array}{c}\text { number of } \\
g_{2}^{p} \text { points }\end{array}$ & Ref. \\
\hline E155X & $\mathrm{d}$ & $0.021-0.780$ & $0.8-8.20$ & 10 & {$[34]$} \\
E99-117 & ${ }^{3} \mathrm{He}$ & $0.327-0.601$ & $2.709-4.833$ & 3 & {$[41]$} \\
\hline
\end{tabular}

Table 3.5: Description of DIS world data set on $g_{2}^{n}$.

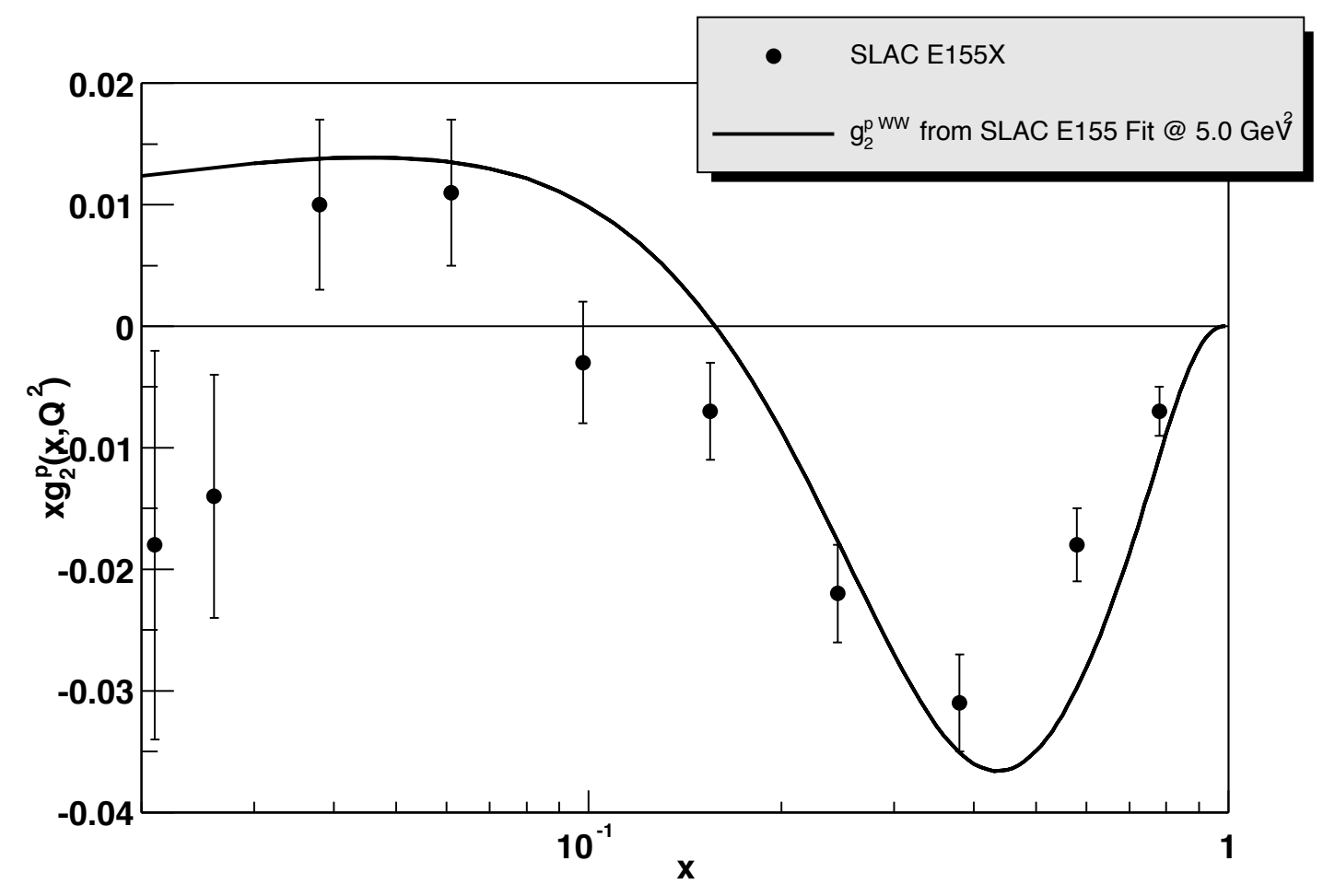

Figure 3.3: $g_{2}^{p}$ from SLAC E155X 


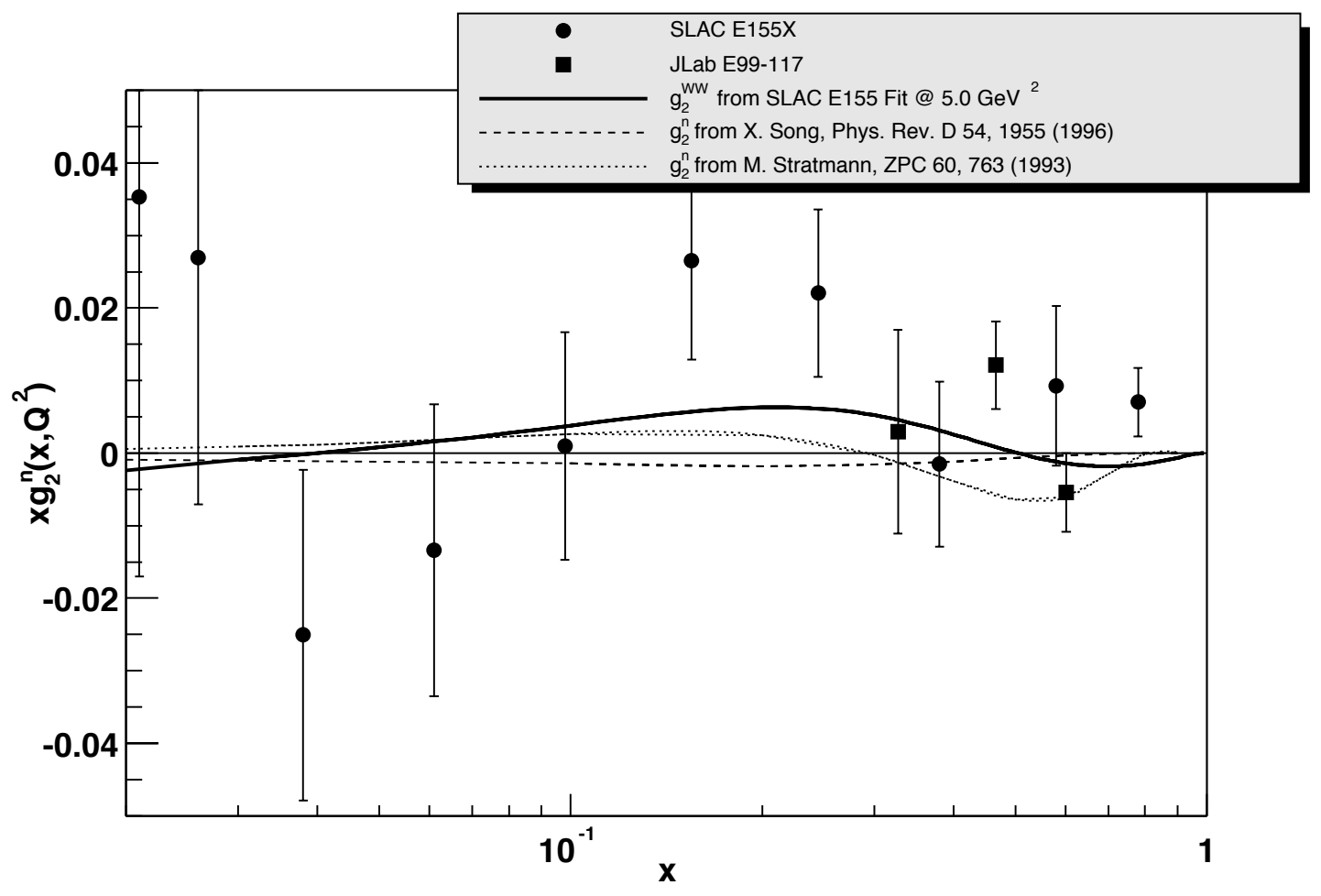

Figure 3.4: $g_{2}^{n}$ from SLAC E155X and JLab E99-117 


\section{CHAPTER 4}

\section{Experimental Overview}

\section{$\underline{4.1}$ Goals of E97-103}

\subsubsection{Physics goals}

The goal of Jefferson Lab experient E97-103 was to measure the spin-structure function $g_{2}^{n}$ at five points in the deep inelastic scattering region. The five kinematic points are given in Table 4.1. The points were chosen to be at an $x \approx 0.2$ and centered at a $Q^{2} \approx 1.0 \mathrm{GeV}^{2}$. The value of $x$ was chosen because of the large value of $g_{2}^{\mathrm{WW}}$ and it was kept nearly constant to be able to isolate the $Q^{2}$ dependence. The $Q^{2}$ was chosen so that it would be in the deep inelastic scattering regime, but at the lowest possible $Q^{2}$ so that the higher twist effects would be larger.

The most obvious way to measure $g_{2}$ is to measure the polarized cross section differences in Eq. 2.21 and Eq. 2.23 with a polarized beam and polarized target. However, cross section measurements are complicated by the need to understand the acceptance and detector efficiencies. It is also possible to measure asymmetries and use the world unpolarized data to circumvent this problem.

\begin{tabular}{cccccc}
\hline $\mathrm{E}(\mathrm{GeV})$ & $\mathrm{E}^{\prime}(\mathrm{GeV})$ & $\theta$ (degrees) & $Q^{2}(\mathrm{GeV})^{2}$ & $x$ & $\mathrm{~W}(\mathrm{GeV})$ \\
\hline 3.465 & 1.595 & 18.39 & 0.565 & 0.161 & 1.97 \\
4.598 & 2.281 & 15.55 & 0.768 & 0.177 & 2.11 \\
4.598 & 1.983 & 18.38 & 0.930 & 0.189 & 2.20 \\
5.727 & 2.622 & 15.61 & 1.11 & 0.190 & 2.37 \\
5.727 & 2.264 & 18.34 & 1.32 & 0.203 & 2.46 \\
\hline
\end{tabular}

Table 4.1: A listing of the acceptance-averaged kinematics for E97-103 
If one defines the cross section differences as:

$$
\Delta \sigma_{\|}=\frac{1}{2}\left(\frac{d \sigma^{\uparrow \Uparrow}}{d E d \Omega}-\frac{d \sigma^{\downarrow \uparrow}}{d E d \Omega}\right), \Delta \sigma_{\perp}=\frac{1}{2}\left(\frac{d \sigma^{\uparrow \Rightarrow}}{d E d \Omega}-\frac{d \sigma^{\downarrow \Rightarrow}}{d E d \Omega}\right)
$$

and the sum:

$$
\sigma_{0}=\frac{1}{2}\left(\frac{d \sigma^{\uparrow \Uparrow}}{d E d \Omega}+\frac{d \sigma^{\downarrow \Uparrow}}{d E d \Omega}\right)=\frac{1}{2}\left(\frac{d \sigma^{\uparrow \Rightarrow}}{d E d \Omega}+\frac{d \sigma^{\downarrow \Rightarrow}}{d E d \Omega}\right)
$$

the longitudinal and transverse asymmetries are defined as

$$
A_{\|}=\frac{\Delta \sigma_{\|}}{\sigma_{0}}, A_{\perp}=\frac{\Delta \sigma_{\perp}}{\sigma_{0}} .
$$

Measuring asymmetries is easier than measuring cross sections since the acceptance, and often the efficiencies, cancel in the asymmetries. While the structure functions can't be extracted directly from the asymmetries, the unpolarized data taken on the proton and deuterium covers a large kinematic range and can be used to calculate $\sigma_{0}$. By combining $\sigma_{0}, A_{\|}$and $A_{\perp}$, one can extract the structure functions $g_{1}$ and $g_{2}$.

Experiment E97-103 was performed in such a way that the structure functions could be extracted either by measuring just the asymmetries or by doing the full cross section analysis. This dissertation is limited to the asymmetry analysis.

An additional complication is that E97-103 measures the spin structure functions of polarized ${ }^{3} \mathrm{He}$ and not the neutron. The method of measuring the cross section or asymmetry is the same, but one must make the additional step of making a correction for the nuclear effects.

\subsubsection{Asymmetry method}

The formulas for calculating the structure functions $g_{1}\left(x, Q^{2}\right)$ and $g_{2}\left(x, Q^{2}\right)$ are

$$
\begin{aligned}
& g_{1}\left(x, Q^{2}\right)=\frac{F_{1}\left(x, Q^{2}\right)}{D^{\prime}}\left[A_{\|}+A_{\perp} \tan \theta / 2\right] \\
& g_{2}\left(x, Q^{2}\right)=\frac{F_{1}\left(x, Q^{2}\right)}{D^{\prime}} \frac{y}{2 \sin \theta}\left[-A_{\|} \sin \theta+A_{\perp} \frac{E+E^{\prime} \cos \theta}{E^{\prime}}\right]
\end{aligned}
$$


where

$$
\begin{aligned}
D^{\prime} & =\frac{(1-\epsilon)(2-y)}{y\left(1+\epsilon R\left(x, Q^{2}\right)\right)} \\
\epsilon & =\frac{1}{1+2\left(1+\left(\nu^{2} / Q^{2}\right)\right) \tan ^{2}(\theta / 2)} .
\end{aligned}
$$

The quantities $F_{1}\left(x, Q^{2}\right)$ and $R\left(x, Q^{2}\right)$ are available from data from the NMC and SLAC experiments [21] on the proton and the deuteron. In addition, Jefferson Lab experiment E94-110 has unpublished data for $F_{1}\left(x, Q^{2}\right)$ and $R\left(x, Q^{2}\right)$ for the proton. These data sets can be used to calculate $F_{1}\left(x, Q^{2}\right)$ for ${ }^{3} H e$. There exists an additional set of unpublished data from the E94-010 experiment on ${ }^{3} \mathrm{He}$ that can also be used to obtain the unpolarized cross section.

It should be mentioned that these are the physics asymmetries that assume a pure ${ }^{3} \mathrm{He}$ target, $100 \%$ beam polarization and $100 \%$ target polarization. Of course, this is not the case in a real experimental situation. The physics asymmetries are derived from the raw experimental asymmetries using

$$
A_{\|}=\frac{A_{\|}^{\text {raw }}}{f P_{t} P_{b}}, A_{\perp}=\frac{A_{\perp}^{\text {raw }}}{f P_{t} P_{b}}
$$

where $f$ is the fraction of the total number of events that came from ${ }^{3} \mathrm{He}$ (known as the dilution factor), $P_{t}$ is the average target polarization and $P_{b}$ is the average beam polarization.

The measured asymmetries are calculated from the number of events within certain acceptance cuts normalized to the number of incident electrons for each helicity using

$$
A^{\mathrm{raw}}=\frac{\left(N^{+} / \zeta^{+} Q^{+}\right)-\left(N^{-} / \zeta^{-} Q^{-}\right)}{\left(N^{+} / \zeta^{+} Q^{+}\right)+\left(N^{-} / \zeta^{-} Q^{-}\right)}
$$

where $N^{ \pm}$is the number of events within acceptance cuts with helicity $\pm 1, Q^{ \pm}$is the accumulated beam charge of helicity \pm 1 , and $\zeta^{ \pm}$is a dead-time correction defined as:

$$
\zeta=\frac{\text { Number of events recorded by the DAQ }}{\text { Number of events that trigger the detector electronics }} \text {. }
$$




\begin{tabular}{|c|c|c|}
\hline \hline Quantity & Description & Source of Measurement \\
\hline \hline$N^{+}, N^{-}$ & $\begin{array}{c}\text { number of electrons in } \\
\text { acceptance cuts }\end{array}$ & HRS detector packages \\
\hline$Q^{+}, Q^{-}$ & Accumulated charge & BCMs \\
\hline$\zeta^{+}, \zeta^{-}$ & Dead time correction & DAQ and scalers \\
\hline$f$ & dilution factor & $\begin{array}{c}\text { reference cell data } \\
\text { and cell fill data }\end{array}$ \\
\hline$P_{t}$ & target polarization & NMR,EPR \\
\hline$P_{b}$ & beam polarization & Møller and Compton polarimeters \\
\hline$E$ & beam energy & Arc and Ep measurements \\
\hline$E^{\prime}$ & $\begin{array}{c}\text { scattered electron } \\
\text { energy }\end{array}$ & spectrometer settings \\
\hline$\theta$ & scattering angle & spectrometer survey \\
\hline \hline
\end{tabular}

Table 4.2: Experimental quantities needed for measurements of $g_{2}^{n}$.

The dead-time correction is necessary since the detector electronics can take data much faster than the data acquisition system (DAQ) can record it.

Finally, $g_{2}^{3} \mathrm{He}$ needs to be converted to $g_{2}^{n}$. This is done using the nuclear correction from $[17]$ :

$$
g_{2}^{n}=\frac{1}{P_{n}+0.056}\left[g_{2}^{3} \mathrm{He}+\left(0.014-2 P_{p}\right) g_{2}^{p}\right]
$$

where $P_{n}$ and $P_{p}$ are the effective neutron and proton polarizations in ${ }^{3}$ He. The structure function $g_{2}^{p}$ is another quantity that must be acquired from world data.

A summary of all the experimental quantities that are needed to measure $g_{2}^{n}$ is provided in Table 4.1.2. The rest of this dissertation will explain how these quantities were measured and their uncertainties were determined and present and discuss the results for $g_{2}^{n}$.

\section{$\underline{4.2 \quad \text { Experimental Setup }}$}

Experiment E97-103 took place at Jefferson Lab in experimental Hall A from 1 Aug 2001 to 17 Sep 2001. It used a polarized ${ }^{3} \mathrm{He}$ gas target and two symmetric high-resolution spectrometers (HRS). Each spectrometer was equipped with the 


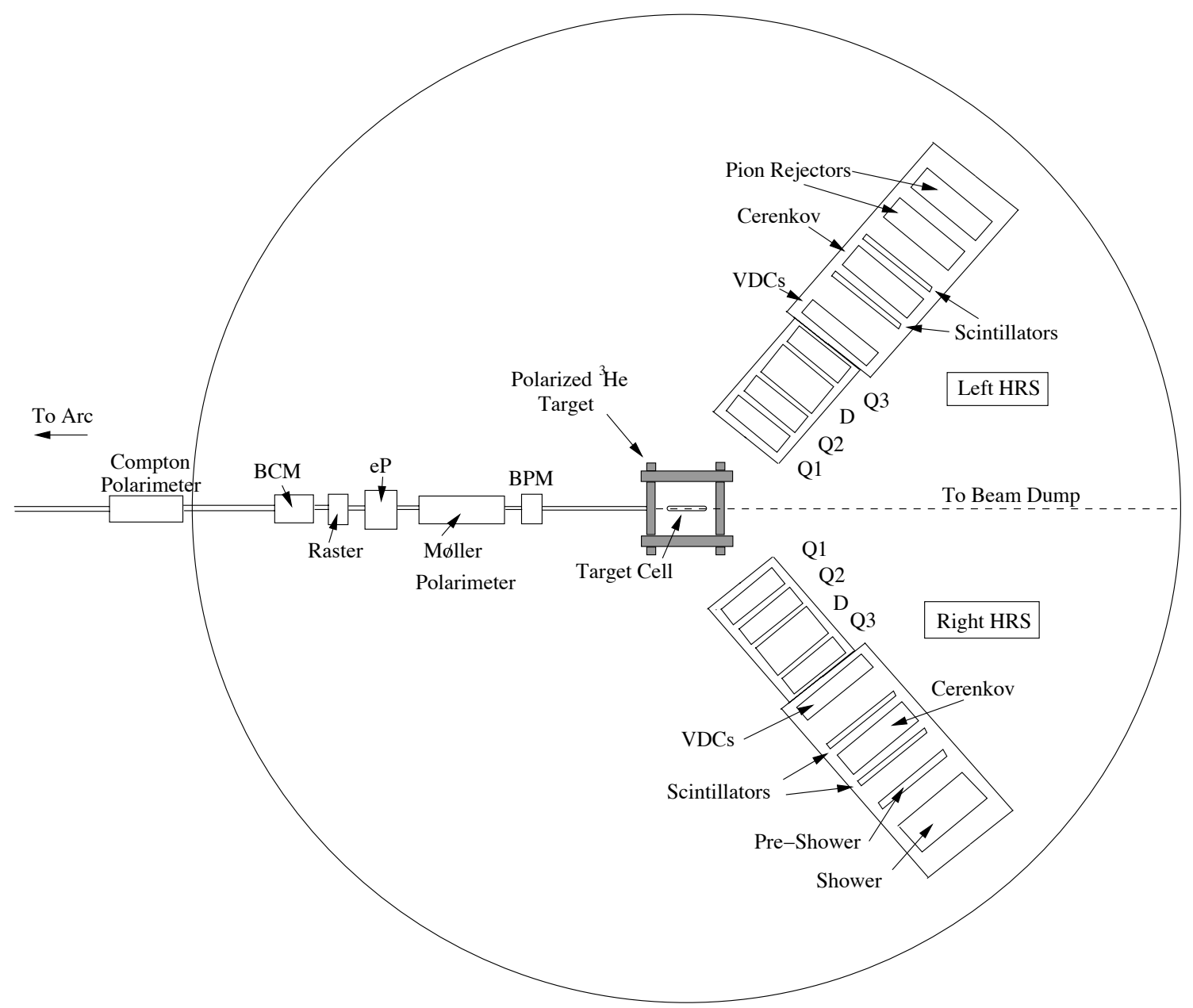

Figure 4.1: A schematic of the spectrometer, target and beam line apparatus in Hall A. 
standard Hall A detector package used here for identifying and characterizing scattered electrons. The Hall A beam line was used with the standard set of equipment for measuring beam energy, polarization and position. A diagram of the Hall A set-up is shown in Fig. 4.1.

The details of the beam line apparatus, the polarized ${ }^{3} \mathrm{He}$ target and spectrometers will be given in the following chapters. 


\section{CHAPTER 5}

\section{The Electron Beam and Beam line Apparatus}

\subsection{Jefferson Lab and CEBAF}

\subsubsection{The accelerator facility}

The experiment E97-103 took place in experimental Hall A at Jefferson Laboratory in Newport News, VA. The continuous electron beam accelerator facility (CEBAF) at Jefferson Laboratory is a polarized electron accelerator specifically designed to study strong interaction physics. It is capable of sending electron beams simultaneously to three experimental end stations, known as Hall A, Hall B and Hall C. Jefferson Lab was a natural choice for E97-103 since the experiment required high luminosity, high beam polarization and enough beam energy to reach the deep inelastic scattering region.

The accelerator, shown in Fig. 5.1, consists of an injector, two linear accelera-

tors (known as linacs) and two sets of recirculation (ARC) magnets. The injector transports electrons from the injector source to the north linac, accelerating them to $45 \mathrm{MeV}$ in the process. The north linac then can accelerate the electrons up to 600 $\mathrm{MeV}$. After passing through the north linac the electrons are transported by a set of recirculation magnets where they enter the south linac. The electrons are given an additional acceleration of up to $600 \mathrm{MeV}$. After the south linac, the electrons can either enter another set of recirculating magnets and be given an additional acceleration by the linacs or enter one of the experimental halls. The electrons can be circulated through the linacs 1-5 times for a energy range of 0.6-5.7 GeV [42].

Each of the cryomodules that make up the linacs consist of eight super-conducting 


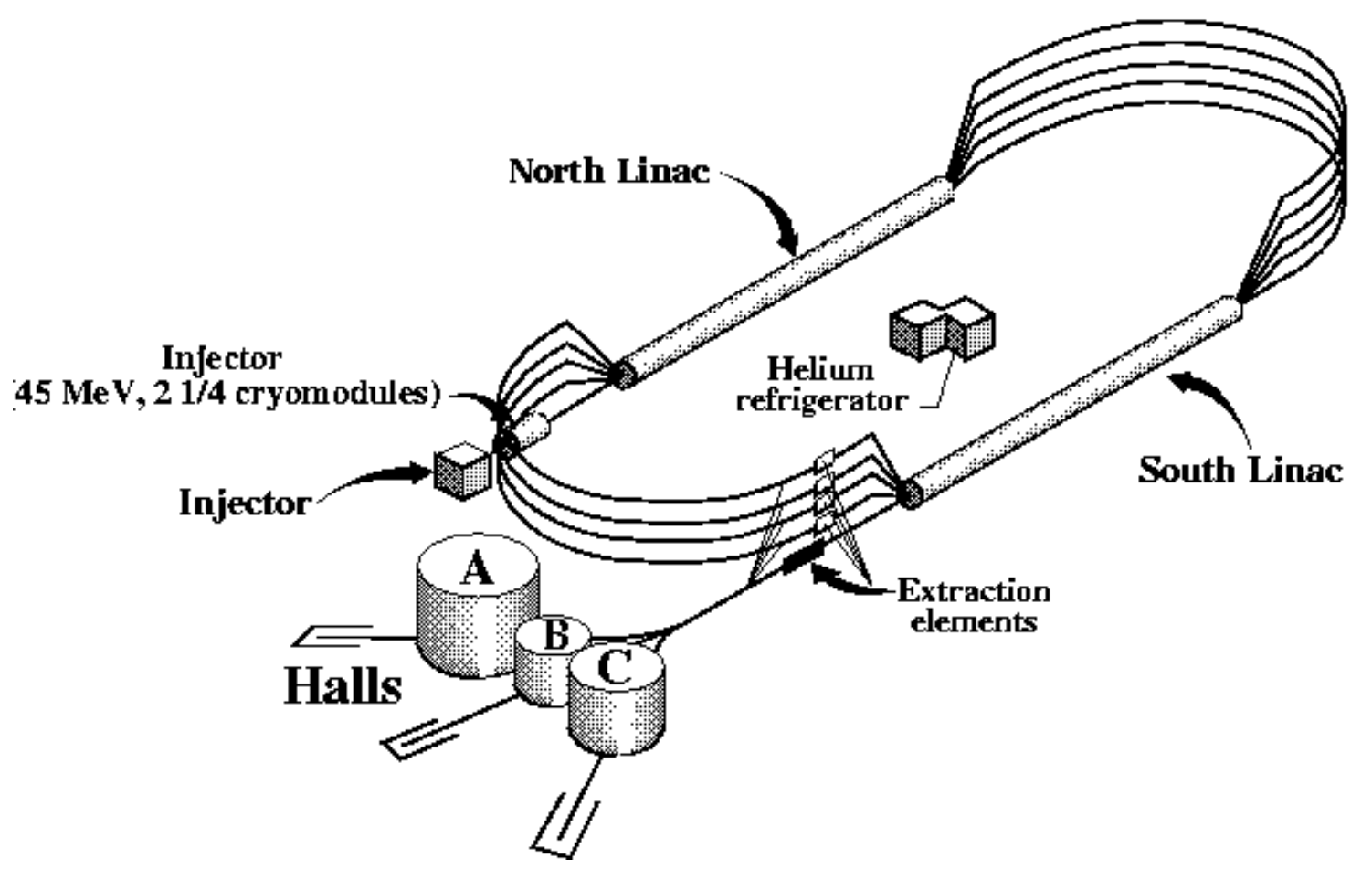

Figure 5.1: The electron accelerator facility at Jefferson Lab.

niobium cavities. The niobium cavities are maintained in a $2.0 \mathrm{~K}$ liquid helium bath to maintain their superconducting properties. The superconductivity allows more power to be applied the cavity without heat loss due to resistance. The electrons are accelerated by $1497 \mathrm{MHz}$ RF oscillations in the cavities. The accelerator divides these oscillations into three $499 \mathrm{MHz}$ bunches, one for each experimental hall [42]. The bunches are separated after the south linac by an RF separator and sent to the appropriate hall. Because of this system, each hall can operate at different beam energy and current.

\subsubsection{The polarized source}

The injector optical source consists of a laser, a linear polarizer, a removable half-wave plate, a Pockels cell, a rotatable half-wave plate and the photocathode. A diagram of these components is shown in Fig. 5.2. The laser is the source of photons needed to excite the electrons at the photocathode. The linear polarizer 


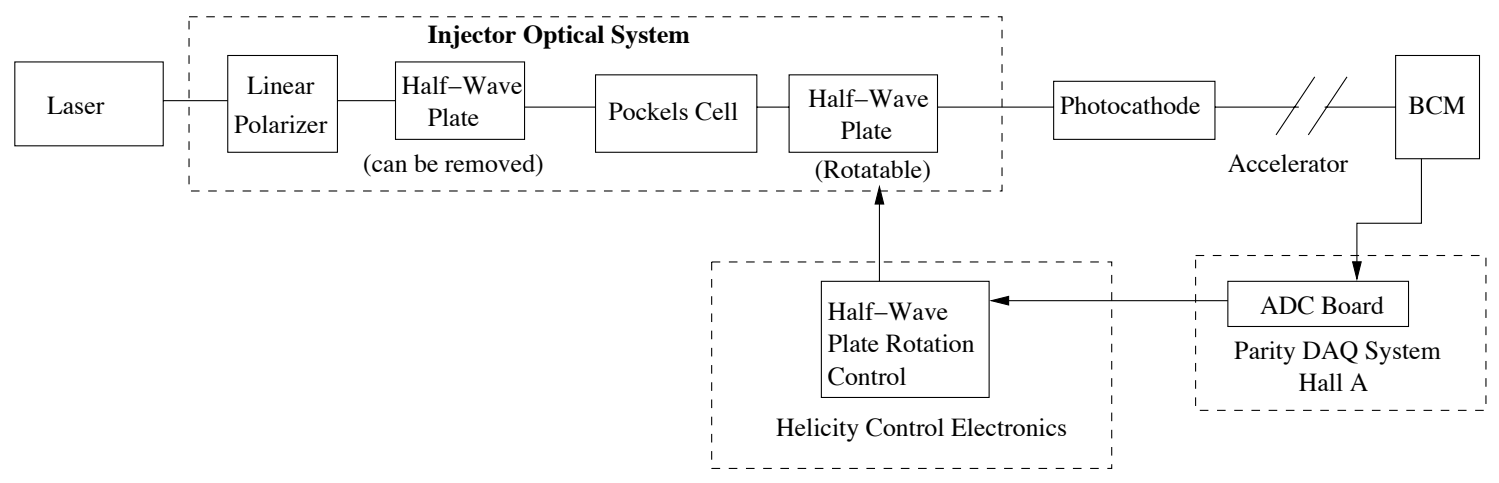

Figure 5.2: A diagram of the helicity feedback system used to control charge asymmetry.

converts the unpolarized light to linearly polarized light. The removable half-wave plate is occasionally inserted in the system to change the sign of the helicity (positive helicity bunches become negative helicity bunches and vice versa). By running with and without the removable half-wave plate, many systematic errors cancel.

The Pockels cell is a voltage-controlled crystal used to convert the linearly polarized light to circularly polarized light. The helicity of the polarized light is controlled by voltage inputs to the Pockels cell which are in turn controlled by the helicity electronics. A rotatable half-wave plate is used to compensate for charge asymmetries due to residual linear polarization that can be analyzed by the photocathode. Finally, the polarized light illuminates the photocathode.

Polarized electrons for CEBAF are created from a GaAs cathode. This cathode is built on a GaAs substrate. Layers of different materials containing GaAs are grown on the substrate as shown in Fig. 5.3. The top layer is made of pure GaAs. The layer directly below it is made of $\mathrm{GaAs}_{0.72} \mathrm{P}_{0.28}$ (a GaAs crystal with $28 \%$ of the arsenic replaced with phosphorus). The lattice spacing of the $\operatorname{GaAs}_{0.72} \mathrm{P}_{0.28}$ is shorter (5.5968 $\AA$ ) than pure GaAs $(5.6533 \AA)$. This causes a strain on the pure GaAs layer and induces an energy gap between electrons in the $\mathrm{P}_{3 / 2}, \mathrm{~m}_{j}= \pm 3 / 2$ states and the $\mathrm{P}_{3 / 2}, \mathrm{~m}_{j}= \pm 1 / 2$ states [43].

The seperation of the electron states allows excitation of electrons in a speciific 


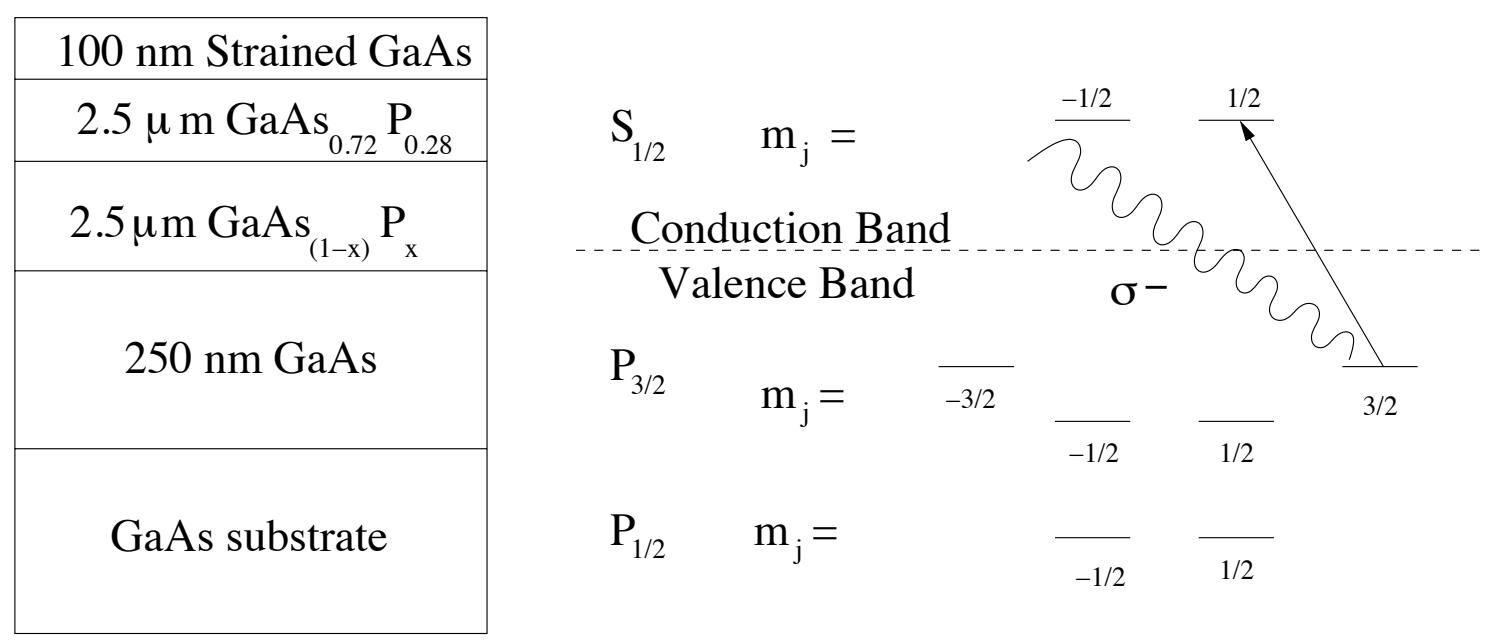

Figure 5.3: Two diagrams of the GaAs cathode used as a source of polarized electrons at $C E B A F$. The boxes on the left represent the different GaAs combinations that make up the photo-cathode. The diagram on the right shown circularly polarized light exciting an electron in the layer of strained GaAs.

$m$ state. By applying left-handed circularly polarized laser light (helicity $=-1$ ), electrons from the $\mathrm{P}_{3 / 2}, \mathrm{~m}_{j}=3 / 2$ state can be excited to $\mathrm{S}_{1 / 2}, \mathrm{~m}_{j}=1 / 2$ state of the conduction band. From there the polarized electrons can escape through the surface into the surrounding vacuum. The electrons that escape by this process will all have the same polarization since only electrons from the $\mathrm{P}_{3 / 2}, \mathrm{~m}_{j}=3 / 2$ can be excited by the circularly polarized light. The same is true of right-handed circularly polarized laser light (helicity $=+1$ ) and electrons in the $\mathrm{P}_{-3 / 2}, \mathrm{~m}_{j}=-3 / 2$ state.

The polarized source can produce $200 \mu \mathrm{A}$ of current split between the three halls. The beam polarization is regularly about $75 \%$ and often exceeds $80 \%$. The maximum beam into Hall A is over $100 \mu \mathrm{A}$, but the beam current for E97-103 never exceeded $15 \mu \mathrm{A}$ due to constraints arising from the polarized ${ }^{3} \mathrm{He}$ target.

\subsection{Measuring the beam energy}

\subsubsection{Arc energy measurements}

The Arc energy measurement is one of two independent methods of measuring the electron beam energy in Hall A. The method extracts the beam energy by 


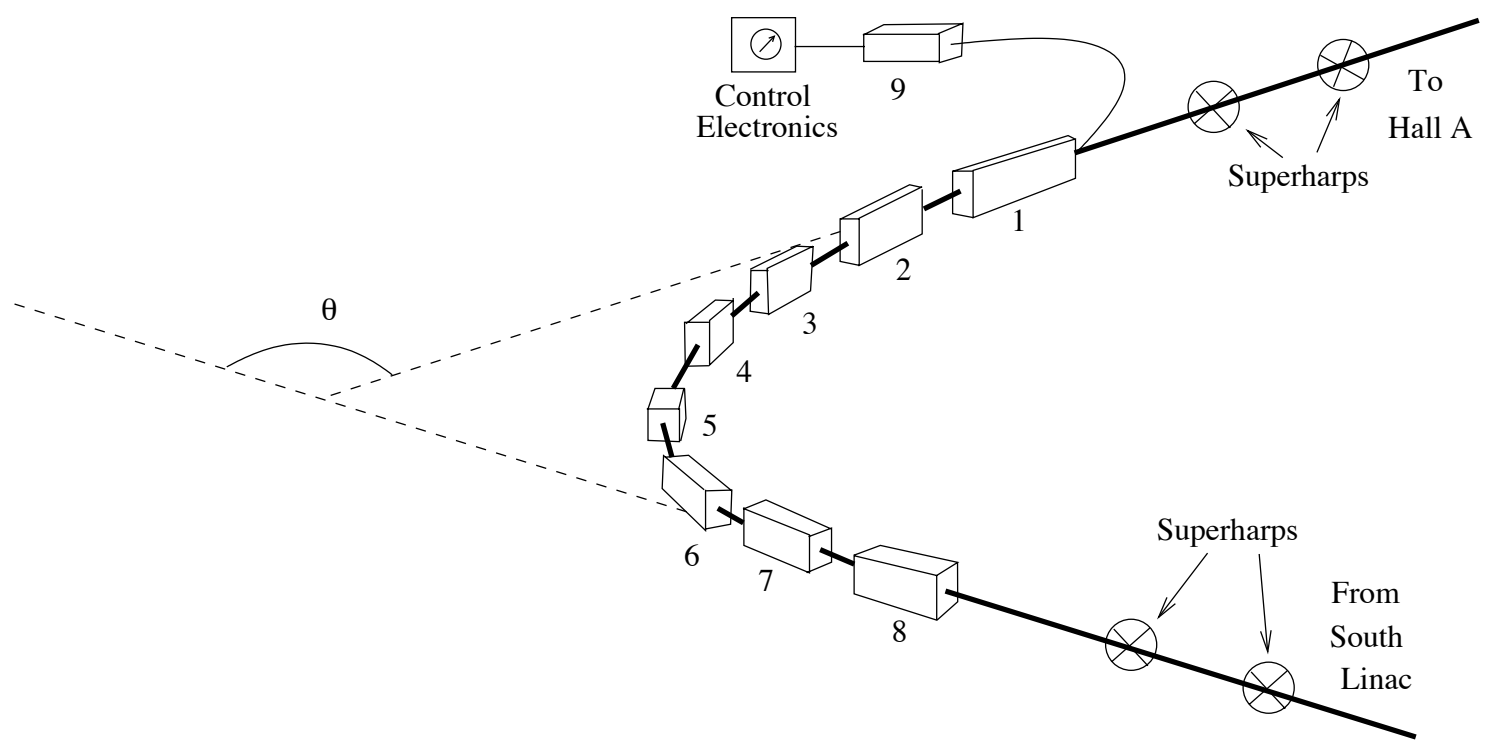

Figure 5.4: The diagram of the equipment used for the Arc energy method.

measuring its deflection in a known magnetic field. Therefore, the technique requires a simultaneous measurement of the deflection of the beam and the integrated magnetic field $\left(\int \vec{B} \cdot \overrightarrow{d l}\right)$ in the arc section of the beam line entering Hall A.

The nominal bend angle of the beam line in this section is $\theta=34.3^{\circ}$ [44]. Deviations from this bend angle are measured by a series of wire scanners known as "superharps". The superharps move a thin wire across the beam. Scattering from the wire is measured by nearby ion chambers. Since the position of the wire is well-known during the beam crossing, the position of the beam can be determined precisely.

The magnetic field is prodced in the 8 dipole magnets in the arc, and their field is calibrated by a ninth dipole connected in series, which is not directly in the beam line, as shown in Fig. 5.4. The beam energy can then be calculated using the formula:

$$
p=c \frac{\int \vec{B} \cdot \overrightarrow{d l}}{\theta}
$$

where $c$ is the speed of light. 


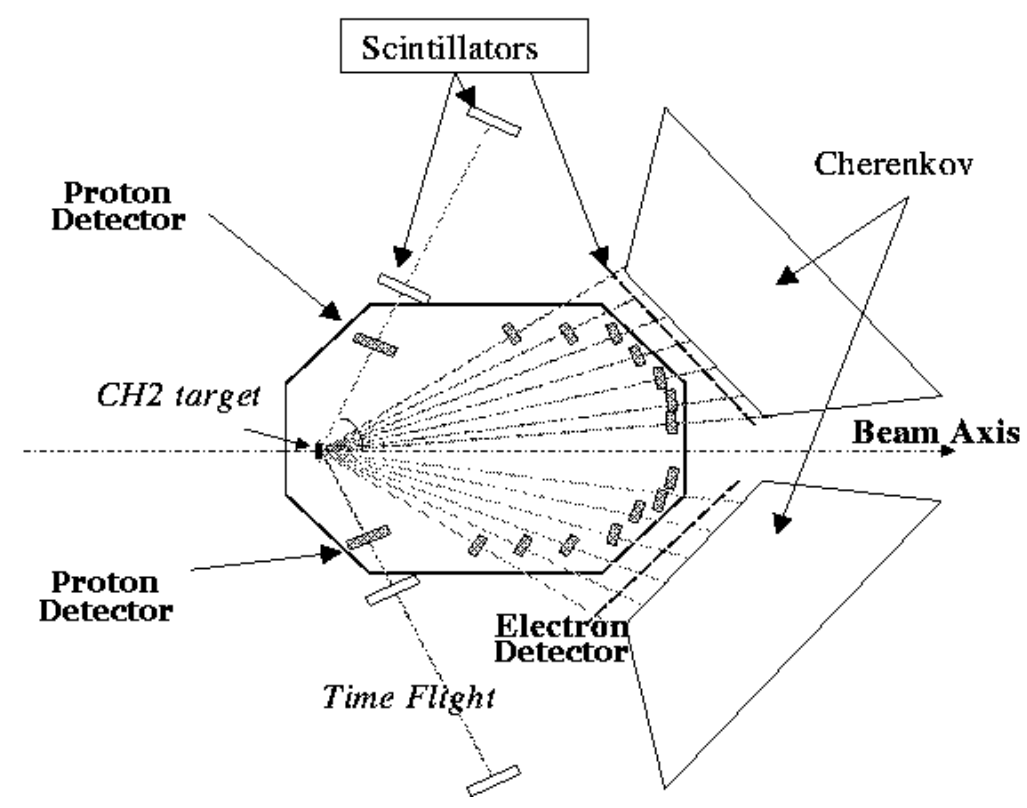

Figure 5.5: A diagram of the eP measurement target and detectors.

\subsection{2 eP energy measurements}

The other method used in Hall A for beam energy measurements is the eP method. This method determines the beam energy by measuring the scattering electron angle $\theta_{e}$, and the recoil proton angle $\theta_{p}$, from the ${ }^{1} \mathrm{H}\left(e, e^{\prime} p\right)$ elastic reaction. The following kinematic formula is used to extract the beam energy [44]:

$$
E=M_{p} \frac{\cos \left(\theta_{e}\right)+\sin \left(\theta_{e}\right) / \tan \left(\theta_{p}\right)-1}{1-\cos \left(\theta_{p}\right)}+O\left(m_{e}^{2}+E^{2}\right) .
$$

where $M_{p}$ is the mass of the proton and $m_{e}$ is the mass of the electron. The second term, $O\left(m_{e}^{2}+E^{2}\right.$ represent higher order terms which are small and ignored in the final calculation.

The diagram in Fig. 5.5 shows the setup of the eP measurement target and detectors. The target is a thin piece of $\mathrm{CH}_{2}$ film. There are two arms that each contain a proton detector and a series of electron detectors. The proton detectors consist of a silicon micro-strip detector for determining position and two scintillators to calculate time of flight. The electron detectors each have a series of 7 micro-strip 


\begin{tabular}{ccccc}
\hline $\begin{array}{c}\text { Date of Energy } \\
\text { Change }\end{array}$ & $\begin{array}{c}\text { Arc } \\
\text { Method }(\mathrm{MeV})\end{array}$ & $\begin{array}{c}\text { eP } \\
\text { Method }(\mathrm{MeV})\end{array}$ & $\begin{array}{c}\text { Tiefenback } \\
\text { Method }(\mathrm{MeV})\end{array}$ & $\begin{array}{c}\text { Average } \\
(\mathrm{MeV})\end{array}$ \\
\hline 4 Aug 2001 & - & $1197.27 \pm 0.47$ & 1196.87 & $1197.27 \pm 0.47$ \\
9 Aug 2001 & $3465.0 \pm 3.0$ & - & 3463.57 & $3465.0 \pm 3.0$ \\
19 Aug 2001 & - & $4598.25 \pm 1.37$ & 4596.98 & $4598.25 \pm 1.37$ \\
15 Sep 2001 & $5728.1 \pm 2.0$ & $5726.0 \pm 1.1$ & 5727 & $5727.1 \pm 1.1$ \\
\hline
\end{tabular}

Table 5.1: List of energy measurements made for E97-103. The Tiefenback method is included only for comparison and is not used in the average.

detectors for covering a wide range of energies. The electron detectors also each have a Cerenkov detector that improves electron identification.

\subsubsection{Beam energy used for E97-103}

Both the arc method and eP method for measuring the beam energy were used in E97-103. The values measured for the beam energies used in the experiment are listed in table 5.1. This table also lists an on-line energy measurement known as the "Tiefenback energy" (named after Jefferson Lab accelerator physicist Michael Tiefenback). This measurement is made using the Hall A Arc $\int B \cdot d l$ value and Hall A Arc beam position monitors. This value can be calculated on-line and is compared to the calibrated values of the Arc measurement.

\subsection{Measuring the beam polarization}

\subsubsection{Møller polarimeter}

E97-103 used two systems to measure the beam polarizations on three orthogonal axes. The Møller polarimeter measures the scattering of polarized electrons from the beam off of polarized atomic electrons in a magnetized foil [44]. The cross section for this scattering depends on the beam and target polarizations $P^{b}$ and $P^{t}$ as shown here:

$$
\sigma \propto\left[1+\sum_{i=X, Y, Z}\left(A_{i i} \cdot P_{i}^{t} P_{i}^{b}\right)\right]
$$



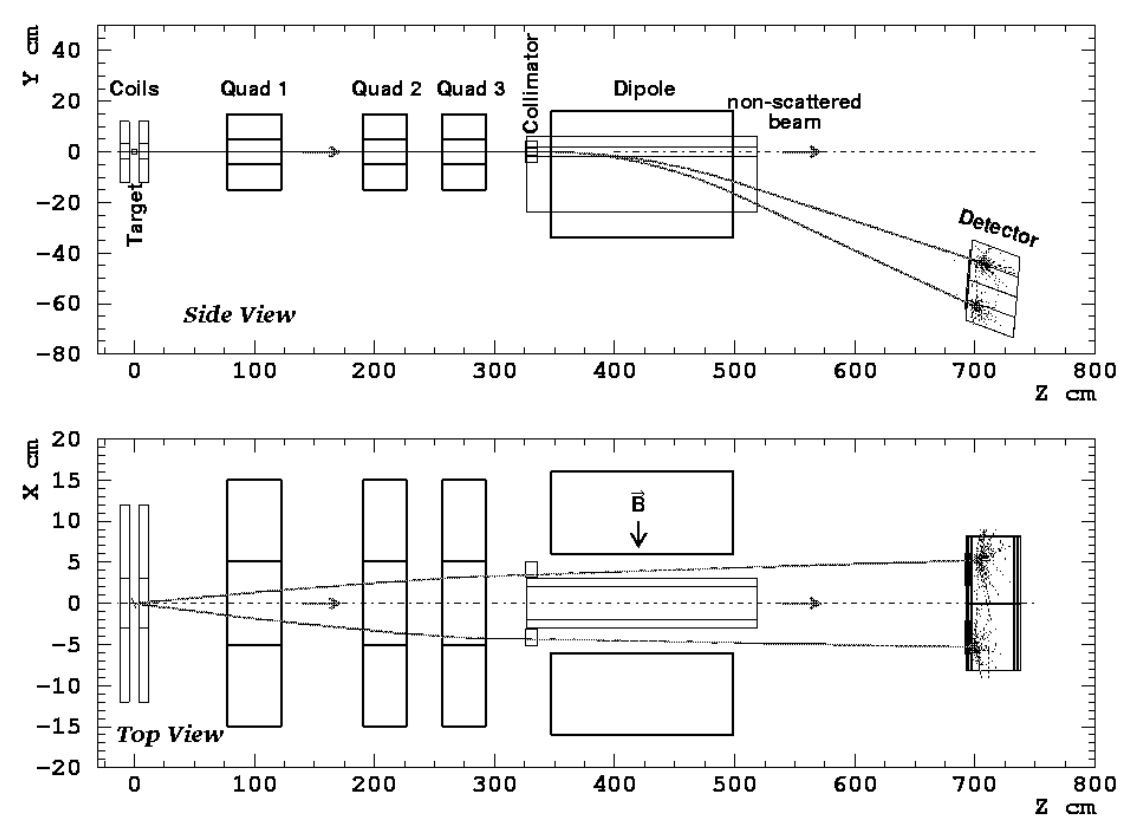

Figure 5.6: A diagram of the Møller polarimeter.

where $i=X, Y, Z$ are the projections of the polarizations. The analyzing power $A$ depends on the scattering angle in the center-of-mass frame, $\theta_{C M}$. [44]. If the beam direction is defined as traveling along the $Z$ axis and the electron scattering happens in the $Z X$ plane then the analyzing power can be written:

$$
\begin{aligned}
A_{Z Z} & =-\frac{\left(7+\cos ^{2} \theta_{C M}\right) \sin ^{2} \theta_{C M}}{\left(3+\cos ^{2} \theta_{C M}\right)^{2}} \\
A_{X X} & =-\frac{\sin ^{4} \theta_{C M}}{\left(3+\cos ^{2} \theta_{C M}\right)^{2}} \\
A_{Y Y} & =-A_{X X}
\end{aligned}
$$

It can be seen from these equations that at $\theta_{C M}=90^{\circ}$ the analyzing power has its maximum value of $7 / 9$.

The Hall A Møller polarimeter uses a ferromagnetic foil in a $24 \mathrm{mT}$ field as a target of polarized electrons. The target can be tilted at various angles to the beam in the horizontal plane to measure both the longitudinal and transverse components of the beam polarization. The asymmetry at angles of $\pm 20^{\circ}$ is measured by two detectors. The target polarization is obtained from offline foil polarization 
measurements [44], and for the supermendur foil used in E97-103 the polarization was $7.95 \pm 0.24 \%$. Knowledge of the target polarization is the largest systematic error in Møller scattering.

The Møller polarimeter uses a magnetic spectrometer consisting of three quadrupole magnets and a dipole magnet as shown in Fig. 5.6. The spectrometer can select electrons from a scattering range of $75^{\circ}<\theta_{C M}<105^{\circ}$ in the horizontal plane and $-5^{\circ}<\phi_{C M}<5^{\circ}$ where $\phi_{C M}$ is azimuthal angle [44]. The polarimeter can be used with beam energies from $0.8 \mathrm{GeV}$ to $6.0 \mathrm{GeV}$.

The detectors of the Møller polarimeter are two lead-glass calorimeter modules. The beam-helicity correlated asymmetries measured in the detectors determine the polarization of the beam. The detectors work in both coincidence mode (only counting electron hits when they strike both detectors simultaneously) and singles mode (measuring electron rates independently). A comparison of the asymmetries measured in coincidence and singles mode reveals that $30 \%$ of the singles events came from a source other than the Møller target. The background in coincidence mode is less than $5 \%$.

\subsubsection{Compton polarimeter}

The second method used in E97-103 for measuring the beam polarization was the Compton polarimeter. Circularly-polarized photons from a laser scatter off the polarized electron beam, and the scattered electrons and scattered photons are detected [44]. The polarization is extracted from the measurement of the counting rate asymmetry of electrons and photons for opposite beam helicities of the electron beam. The Compton measurement, unlike that with the Møller polarimeter, is an effectively passive measurement and can be done while taking production data on the main target.

The Compton polarimeter, as pictured in Fig. 5.7, consists of a magnetic chi- 


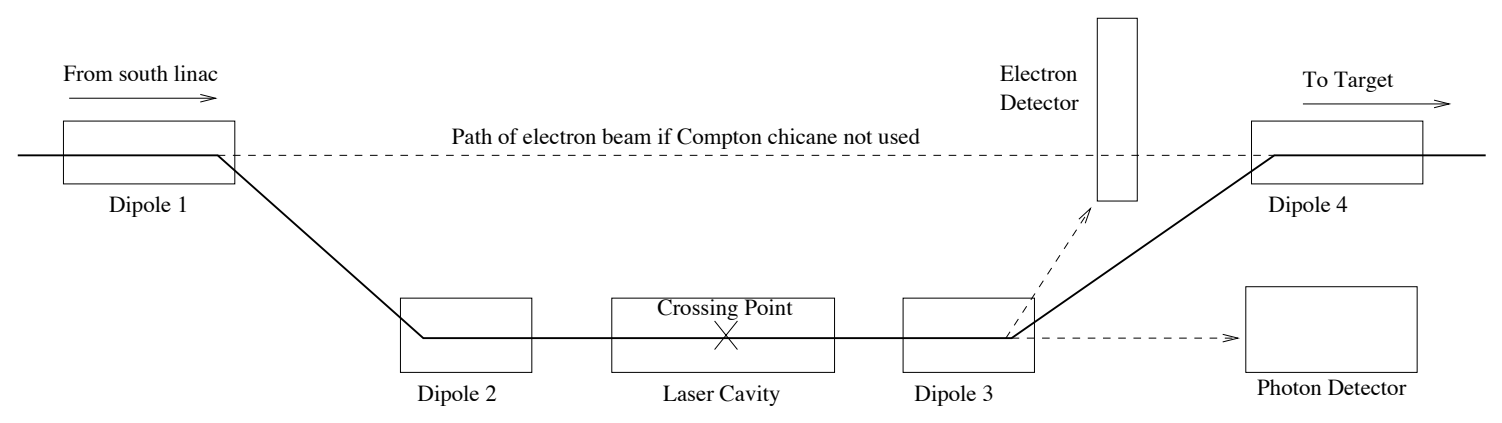

Figure 5.7: Schematic of the Compton apparatus

cane, a photon source, an electromagnetic calorimeter and an electron detector. The chicane bends the beam vertically by four dipole magnets so that the photon beam provided by the laser can cross the electron beam and the scattered electrons and photons can be detected. The photons from the laser are backscattered into a calorimeter. A silicon strip electron detector is used to detect the scattered electrons. Electrons that did not interact with the photon beam exit the polarimeter and reach the target.

A resonant Fabry-Pérot cavity is used the amplify the photon beam [45]. The resonance cavity uses two mirrors to amplify a primary $230 \mathrm{~mW}$ CW Nd:YaG laser $(\lambda=1064 \mathrm{~nm})$. An amplification factor of 7300 has been measured corresponding to a photon beam power of $1680 \mathrm{~W}$ inside the cavity [44]. The circular polarization of light has been measured to be $>99 \%$ for both positive and negative helicity states. The helicity of the polarized laser light can be changed by using a rotatable quarter-wave plate.

To maximize the number of scattered photons, the angle at which the photon beam crosses the electron beam must be as small as possible. The nominal crossing angle is $23 \mathrm{mrad}$. The position of the electron beam and the photon beam are adjusted until the maximum rate of Compton events in the detectors is acheived. The measurement can be done using photon singles events, electron singles events or a coincidence measurement of photon and electrons. Measurements taken with 


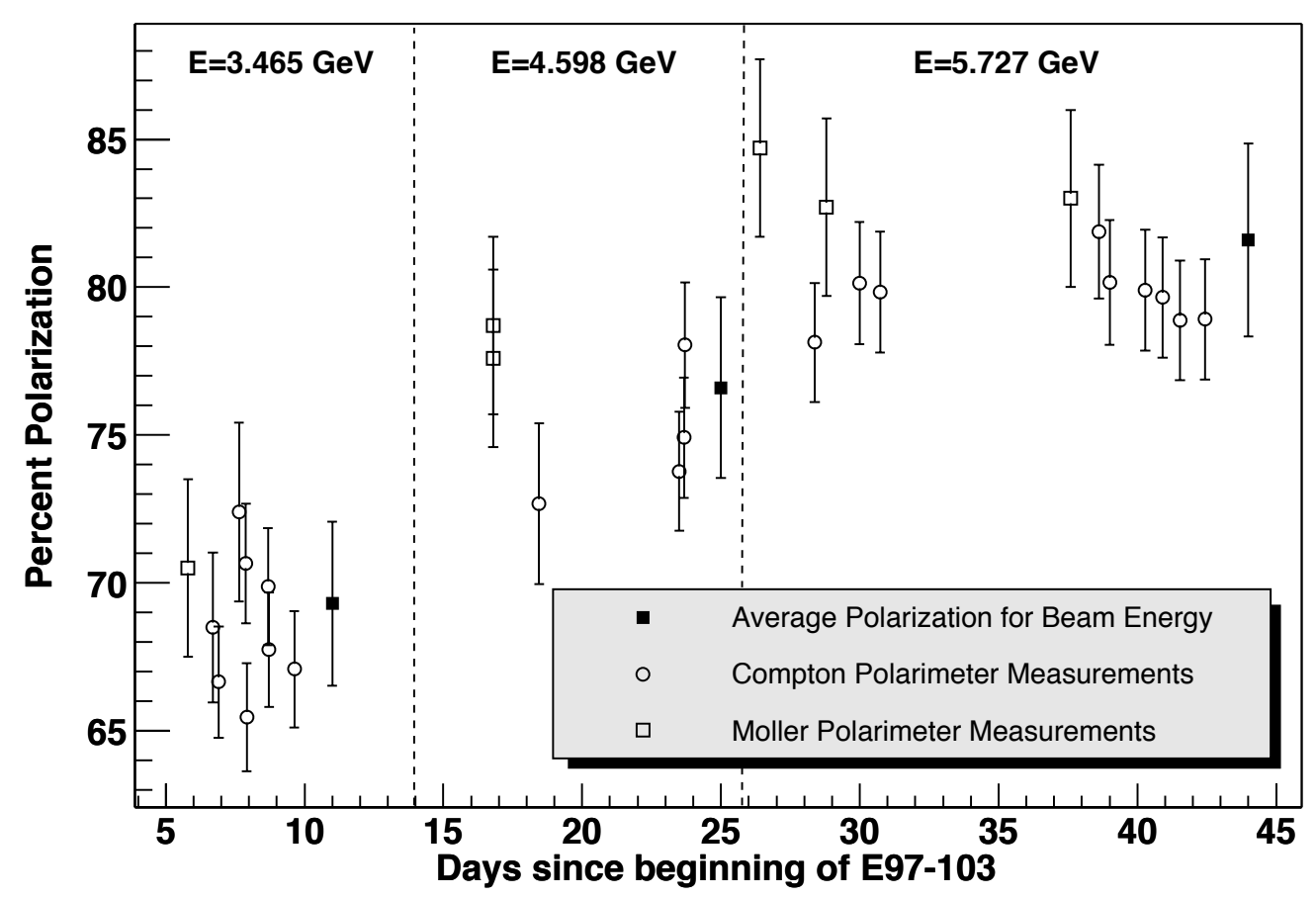

Figure 5.8: A plot of the different beam polarization measurements and their average for each beam energy.

\begin{tabular}{cc}
\hline Beam Energy (GeV) & Polarization (\%) \\
\hline 1.19727 & $83.1 \pm 3.3$ \\
3.4650 & $-69.3 \pm 2.8$ \\
4.59825 & $76.6 \pm 3.1$ \\
5.7271 & $-81.6 \pm 3.3$ \\
\hline
\end{tabular}

Table 5.2: The values used for the polarization for each beam energy are based on a combination of Møller and Compton polarimeter measurements.

the laser beam off reveal a background/signal ratio of as good as 0.05 [44].

Using the position of the electrons in the micro-strip planes and from the amount of light collected, one can calculate the energy of the scattered particles. In coincidence mode this is especially useful, since the photon energies can be determined allowing a calibration of the response functions of the calorimeter to be made. These type of coincidence measurements have the smallest systematic errors. 


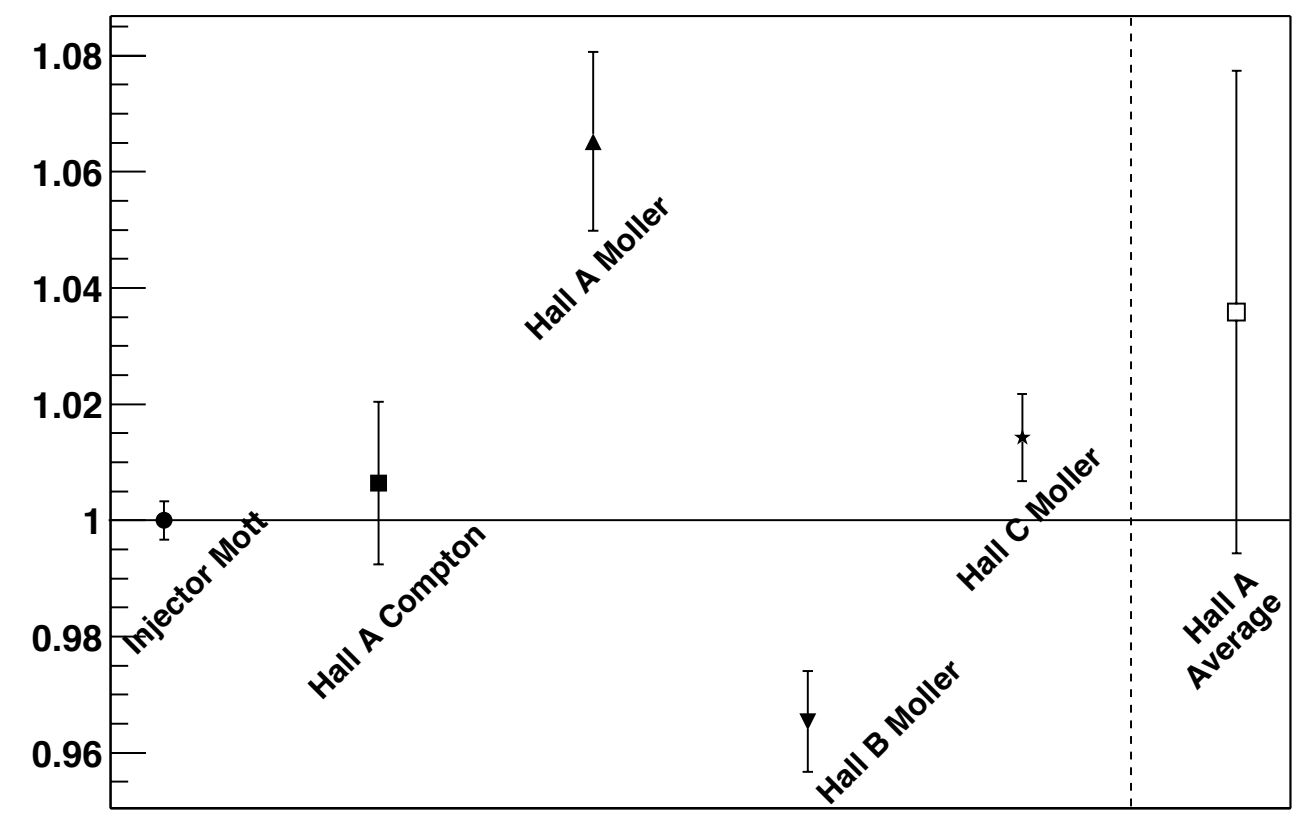

Figure 5.9: The results for a comparison of Jefferson Lab beam polarimeters performed in 2000. The polarization is normalized to the Mott polarimeter. The "Hall A Average" is the value of the polarization using the method used in E97-103.

\subsubsection{Beam polarization for E97-103}

The values used for the polarization are listed in Table 5.2. A plot of the average values compared with the individual Compton and Møller polarimeter measurements is shown in Fig. 5.8.

The errors on the polarization is estimated to be $4 \%$ relative error. This error is based on results of the Spin Dance 2000 measurement [46] and recent experience in Hall A. In the Spin Dance measurements, polarimetry results from all three halls were compared to each other for the same beam polarization. The results in Fig. 5.9 are shown with their expected systematic errors of each polarimeter normalized to the polarization value given by the injector Mott polarimeter. The results show that there is significant discrepancy between the Hall A Møller and the Hall A Compton polarimeters. The "Hall A Average" shown in Fig. 5.9 is the value of the 


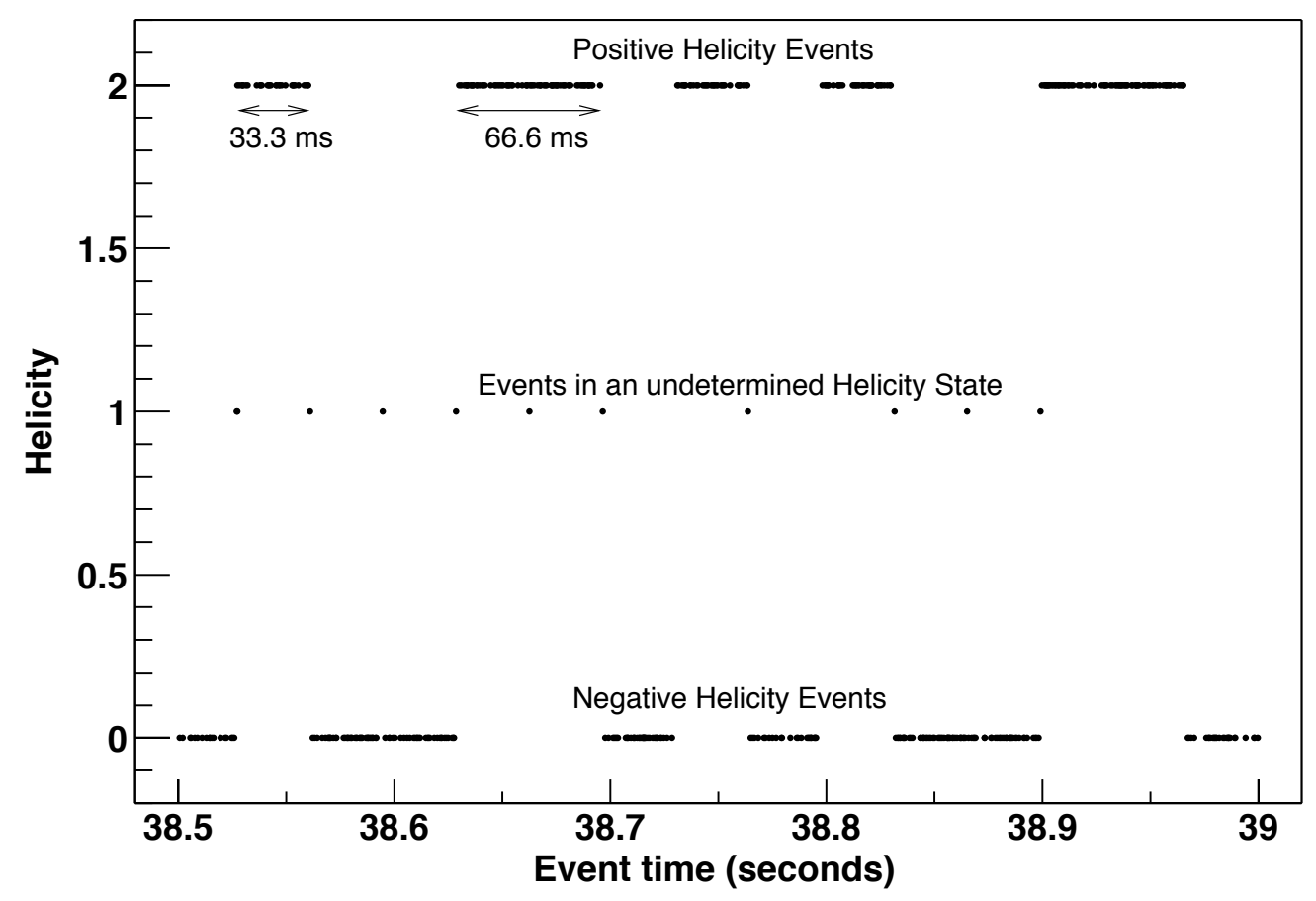

Figure 5.10: A plot of the beam helicity in a half second time span as extracted from reconstructed data. See text for details.

polarization using the average of the Hall A Compton and Hall A Møller and a 4\%

relative error. The average of the Hall A Compton measurements and Hall A Møller measurements are computer separately and averaged.

\subsection{Beam helicity, charge and position}

\subsubsection{Beam helicity}

As can be seen in Eq. 2.21 and Eq. 2.23, the spin-dependent structure functions can be isolated by measuring the difference in cross section correlated with changing beam helicity. The helicity is flipped by a Pockels cell that changes the handedness of the circularly polarized laser light that excites the electrons from the photocathode. The Pockels cell design allows the helicity to be changed pseudo-randomly at a rate of $30 \mathrm{~Hz}$.

An example of the helicity pattern is shown in Fig. 5.10. This plot shows 
the beam helicity in a 0.5 second time span as extracted from reconstructed data. Each point represents an event that triggered the detector system. The events with helicity ' 0 ' occurred during periods of negative beam helicity and helicity '2' events occurred during periods of positive beam helicity. The helicity ' 1 ' events occurred during periods of undetermined helicity. These periods are usually caused by a 0.2 ms "blank off" that is set up in the electronics system to occur every $33.3 \mathrm{~ms}$ to cover up periods when the helicity is changing. This eliminates the number of events assigned the wrong helicity.

The helicity pattern does not simply alternate between positive and negative helicity states regularly (as it would in "toggle" mode). Instead the next state is determined randomly (known as "pseudo-random" mode) which alternates periods of $33.3 \mathrm{~ms}$ and $66.6 \mathrm{~ms}$ in each helicity state. This technique eliminates systematics that could be associated with $30 \mathrm{~Hz}$ noise in the system.

\subsubsection{Measuring Beam Charge}

The amount of charge delivered to the target is an important normalization factor in both the cross section and asymmetry measurements. In Hall A the charge is measured by a passive beam current monitor (BCM). The BCM consists of an Unser monitor, two RF cavities, associated electronics and a data-acquisition system. The setup is displayed in Fig. 5.11 [47].

The Unser monitor is a Parametric Current Transformer which provides an absolute reference for the calibration of the beam [44]. The monitor is calibrated by passing a known current through a wire inside the beam pipe. To maintain this calibration, extensive magnetic shielding and temperature stabilization is required to reduce noise and baseline drift. However, since the Unser monitors output signal drifts if used for a period more than several minutes, it can not be used to continuously monitor the beam current. 


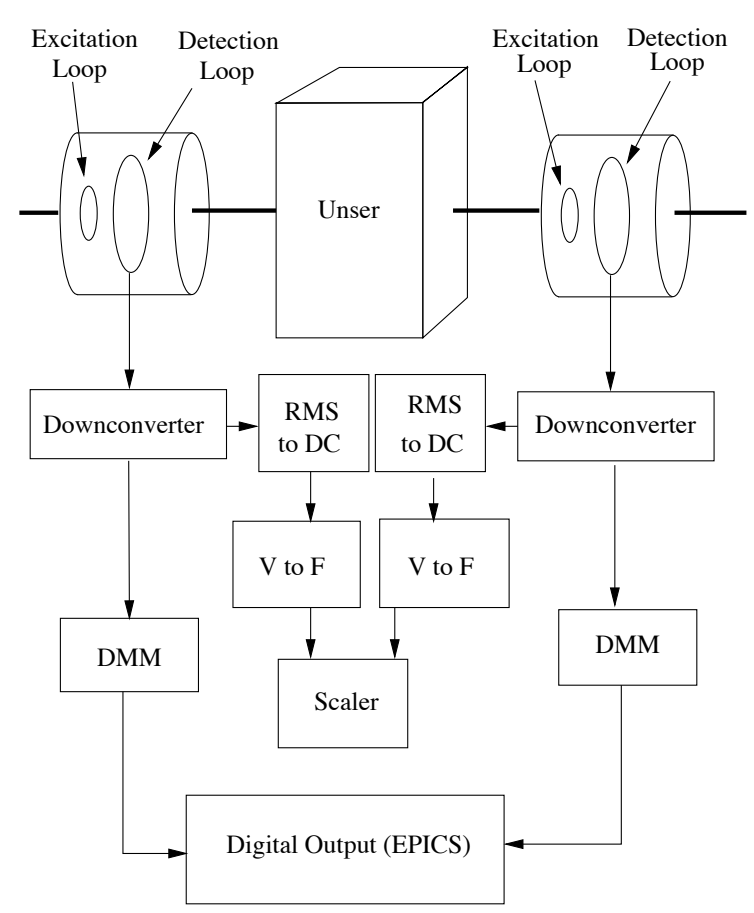

Figure 5.11: Beam Charge Montioring system

Two RF resonance cavity monitors are stainless steel waveguides tuned to the frequency of the charge oscillation of the accelerator cavities $(1497 \mathrm{MHz})$. The voltage output of these monitors is proportional to the current in the beam [44]. The output data from the cavities is sent to two output channels: a sampled channel and an integrated channel.

The sampled channel is sent to a digital multimeter which produces a digital signal that represents the RMS of the signal over one second. These signals are sent via GPIB ports to a computer where the RMS values for both cavities are stored in the EPICS database (this is part of the data acquisition system the will be discussed in a later chapter). The integrated values are sent to an RMS to DC converter followed by a voltage to frequency converter ( $\mathrm{V}$ to $\mathrm{F}$ ). The integrated readings are sent to a scaler which is read by the DAQ and inserted into the data stream every four seconds [44].

The RF cavities are calibrated regularly by running the current from zero to the 


\begin{tabular}{|c|ccc|c|}
\hline \multicolumn{5}{|c|}{ Upstream Cavity } \\
\hline $\begin{array}{c}\text { Ampli- } \\
\text { fication }\end{array}$ & ungated & $\begin{array}{c}\text { Offsets } \\
\text { positive }\end{array}$ & negative & \\
\hline 1 & 92.072596 & 92.21067 & 92.069586 & 1345 \\
3 & 167.05737 & 167.0949 & 166.95239 & 4114 \\
10 & 102.62361 & 102.62498 & 102.46542 & 12515 \\
\hline \multicolumn{5}{|c|}{ Downstream Cavity } \\
\hline Ampli- & \multicolumn{5}{|c|}{ Offsets } & Constant \\
fication & ungated & positive & negative & \\
\hline 1 & 72.190291 & 72.309803 & 72.176298 & 1303 \\
3 & 91.080796 & 91.145456 & 90.984981 & 4114 \\
10 & 199.50698 & 199.57484 & 199.34949 & 12728 \\
\hline
\end{tabular}

Table 5.3: Table of constants used for calculating accumulated charge sent to the target.

maximum current several times, dwelling at each setting for 60 to $90 \mathrm{~s}$. The output from the Unser is used to calibrate the output of the RF cavities. The calibration can be determined down to a current of $1 \mu \mathrm{A}$ with an accuracy of $\leq 0.5 \%$ [44].

The calibration used for E97-103 was performed in January 2001 and is given in Table 5.3. The accumulated charge can be derived from these calibration constants and values from the scalers. The scaler values needed are the helicity gated clock values $\left(t_{h}\right)$ (the helicity gated clock times the amount of time in each helicity state with a $1024 \mathrm{~Hz}$ clock) and the helicity gated BCM scaler reading for the upstream cavity $\left(N_{h}^{u}\right)$ and for the downstream cavity $\left(N_{h}^{d}\right)$. In the case of $t_{h}, N_{h}^{u}$ and $N_{h}^{d}$ there are three types of helicity : positive, negative and ungated, which is the sum of positive and negative. In addition to this, for each helicity of $t_{h}, N_{h}^{u}$ and $N_{h}^{d}$ there are three levels of amplification $(\mathrm{x} 1, \mathrm{x} 3, \mathrm{x} 10)$ that can be used for different ranges of beam current.

This creates a total of 18 possible values for the accumulated charge (2x for 2 cavities, $3 \mathrm{x}$ for three helicities and $3 \mathrm{x}$ for three levels of amplification). All 18 values can be calculated using the following formula with the correct constants from table 
5.3:

$$
Q_{h, a}^{c}=\frac{N_{h, a}^{c} / t_{h}-f_{a, h}}{c_{a}} t_{h}
$$

where $h$ refers to the helicity, $a$ refers to the amplification, $c$ refers to the cavity (upstream or downstream), $f_{a, h}$ refers to the corresponding offset in table $5.3, c_{a, h}$ refers to the corresponding constant in table $5.3, Q_{h, a}^{c}$ is the accumulated charge in time period $t_{h}$ and $N_{h, a}^{c}$ is the accumulated scaler reading for time period $t_{h}$.

For E97-103 the charge was calculated using the 3x amplifier scaler because it is appropriate for current values from 1-20 $\mu \mathrm{A}$. The charge vales for the upstream and downstream cavities were averaged. The formula used to calculate the positive and negative accumulated charge is given by the equations:

$$
\begin{aligned}
& Q_{+}=\frac{1}{2}\left(Q_{+, 3}^{u}+Q_{+, 3}^{d}\right) \\
& Q_{-}=\frac{1}{2}\left(Q_{-, 3}^{u}+Q_{-, 3}^{d}\right)
\end{aligned}
$$

where + and - refer to positive and negative helicity respectively.

\subsubsection{Beam charge asymmetry feedback system}

Due to helicity dependent differences in the injector or in the helicity gating, there can be significantly different amounts of charge in each helicity state. These differences result in a charge asymmetry which is defined by the following formula:

$$
A_{Q}=\frac{Q^{+}-Q^{-}}{Q^{+}+Q^{-}} .
$$

$Q^{+}$and $Q^{-}$are the accumulated charge given by the BCMs. However, these can be broken down further, for purposes of studying the charge asymmetry, into $Q^{+}=$ $I^{+} t^{+}$and $Q^{-}=I^{-} t^{-}$where $I^{ \pm}$is the beam current during \pm helicity states and $t^{ \pm}$ is the time interval of the helicity pulses [41].

The two general categories for sources of beam charge asymmetry are when $t^{+} \neq t^{-}$and when $I^{+} \neq I^{-} . t^{+} \neq t^{-}$is caused by unequal timing the helicity 
generation circuitry. $I^{+} \neq I^{-}$is caused by various sources in the injector source. These include incomplete polarization of the injector laser, imperfections in the Pockels cell or half-wave plate.

The charge asymmetry from either of these categories can be corrected, when calculating the cross section or asymmetry, by normalizing the charge for the positive and negative helicity states independently. However, the charge asymmetry can only be measured confidently to $1 \%$ because of non-linear effects in the BCMs. Also a beam intensity asymmetry affects the beam transport in the accelerator. Therefore, a helicity feedback system was created to minimize the effect of charge asymmetry on the production physics data.

The timing differences in the helicity gates was expected to be small enough not to need significant improvement; therefore the helicity feedback system focused on controlling the intensity differences at the source. The helicity feedback system, as shown in Fig. 5.2 worked by recording the BCM values for a certain time period (on the order of 5 minutes), calculating the charge asymmetry and automatically adjusting a rotatable half-wave plate at the injector source to compensate for this asymmetry.

A separate data-acquisition system, called the "parity DAQ", was set up to control the rotatable half-wave plate. The parity DAQ would record values from the $\mathrm{BCM}$ and calculate the charge asymmetry every five minutes. A new settings for the rotatable half-wave plate would be entered into an EPICS database on a ADC board in Hall A (the one used for the target controls). This database could be read by control systems at the injector which would make the proper rotation of the half-wave plate.

This system exceeded the necessary requirements for the experiment. A charge asymmetry of less than $200 \mathrm{ppm}$ would of been sufficient to suppress the charge asymmetry as a source of systematic error. One concern for this experiment is that 

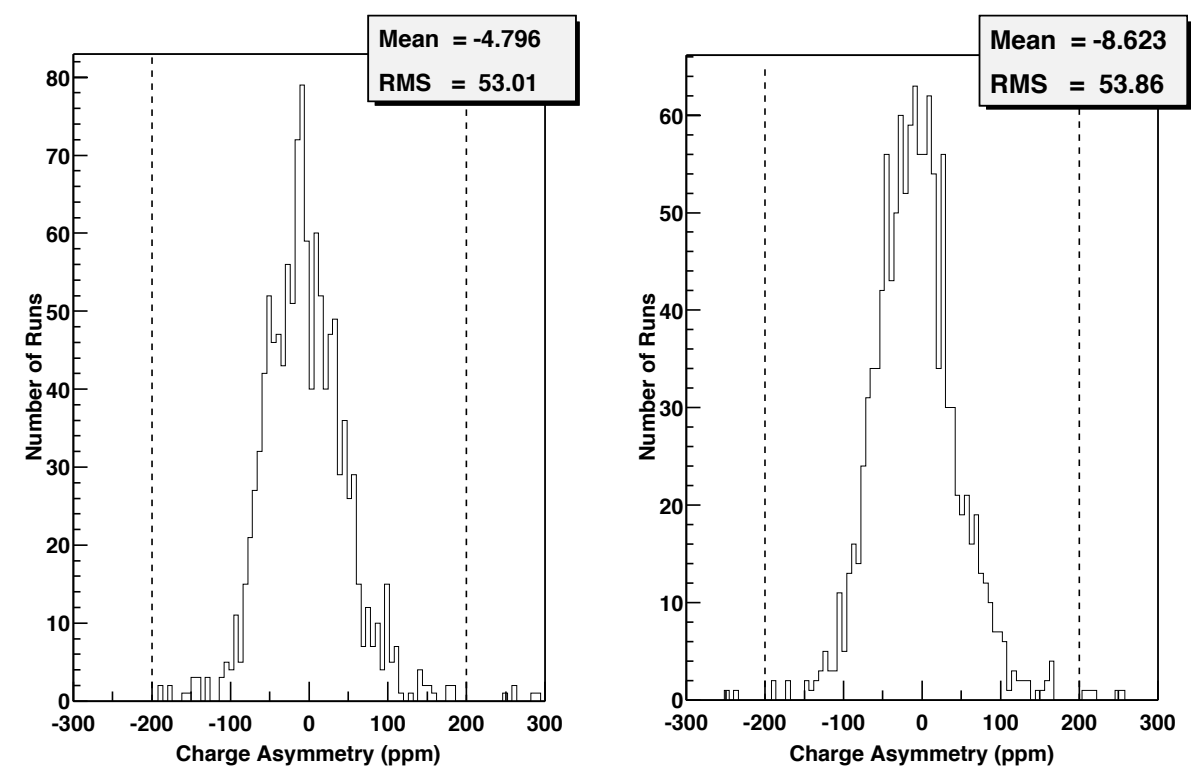

Figure 5.12: The distribution of charge asymmetry for each run. The left histogram is the charge asymmetry with the beam trips and the right histogram is the charge asymmetry with the beam trips cut out.

the beam asymmetry would occasionally be large during periods when the beam was ramping up to the nominal current after a beam trip. This procedure could last up to 30 seconds with an average of 3-4 beam trips per run. These periods can be cut out of the data when calculating the charge asymmetry. Histograms of the charge asymmetry for each run are shown in Fig. 5.12. The left histogram is with the beam trips included in the data and the right histogram is the charge asymmetry distribution with these beam asymmetries cut out. Both show a distribution width around $53 \mathrm{ppm}$, which more than satisfied the design requirements of the experiment.

\subsubsection{Beam position monitors}

Careful measurement of the beam position is necessary to make sure the beam stays on the target and because it can change the values of important kinematic values. Determination of the beam position is made by two beam position monitors (BPMs) located at $7.5 \mathrm{~m}$ (BPMA) and $2.4 \mathrm{~m}$ (BPMB) upstream of the target. The 


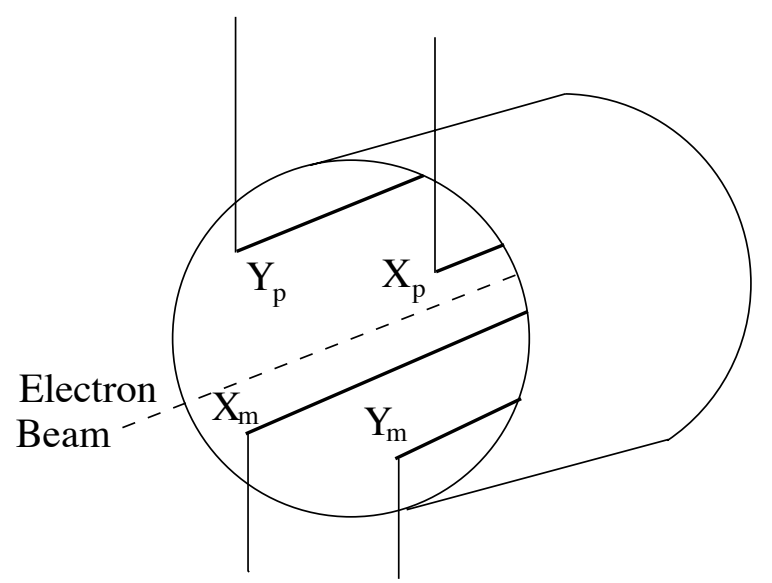

Figure 5.13: A diagram of the beam position monitor

BPMs each consist of four antennae that are tuned to the $1497 \mathrm{MHz}$ oscillation of the accelerator. The voltage output of each attena increases as the beam gets closer to it [48].

The BPMs are calibrated with wire scanners or harps. These are located adjacent to the BPMs. Just like the superharps, used in the Arc energy measurement, the harp measurement moves a wire across the beam line and measures where the wire crosses the beam by scattering into ion chambers. The wire scanners used for the BPMs are regularly surveyed with respect to the coordinate system in Hall A.

The beam position at the target can be calculated using the coordinates derived from the BPMs and the following formulas:

$$
\begin{aligned}
x_{\text {beam }} & =\frac{1}{z_{B}-z_{A}}\left(x_{A} z_{B}-x_{B} z_{A}\right) \\
y_{\text {beam }} & =\frac{1}{z_{B}-z_{A}}\left(y_{A} z_{B}-y_{B} z_{B}\right) \\
\theta_{\text {beam }} & =\frac{x_{B}-x_{A}}{z_{B}-z_{A}} \\
\phi_{\text {beam }} & =\frac{y_{B}-y_{A}}{\sqrt{\left(x_{B}-x_{A}\right)^{2}+\left(z_{B}-z_{A}\right)^{2}}}
\end{aligned}
$$

where $x_{A}$ and $x_{B}$ are the $x$ coordinates determined by the BPMA and BPMB BPMs respectively, $y_{A}$ and $y_{B}$ are the $y$ coordinates determined by the BPMA and BPMB BPMs respectively, $z_{A}$ is $-7.345 \mathrm{~m}$ and $z_{B}$ is $-2.214 \mathrm{~m}$. 

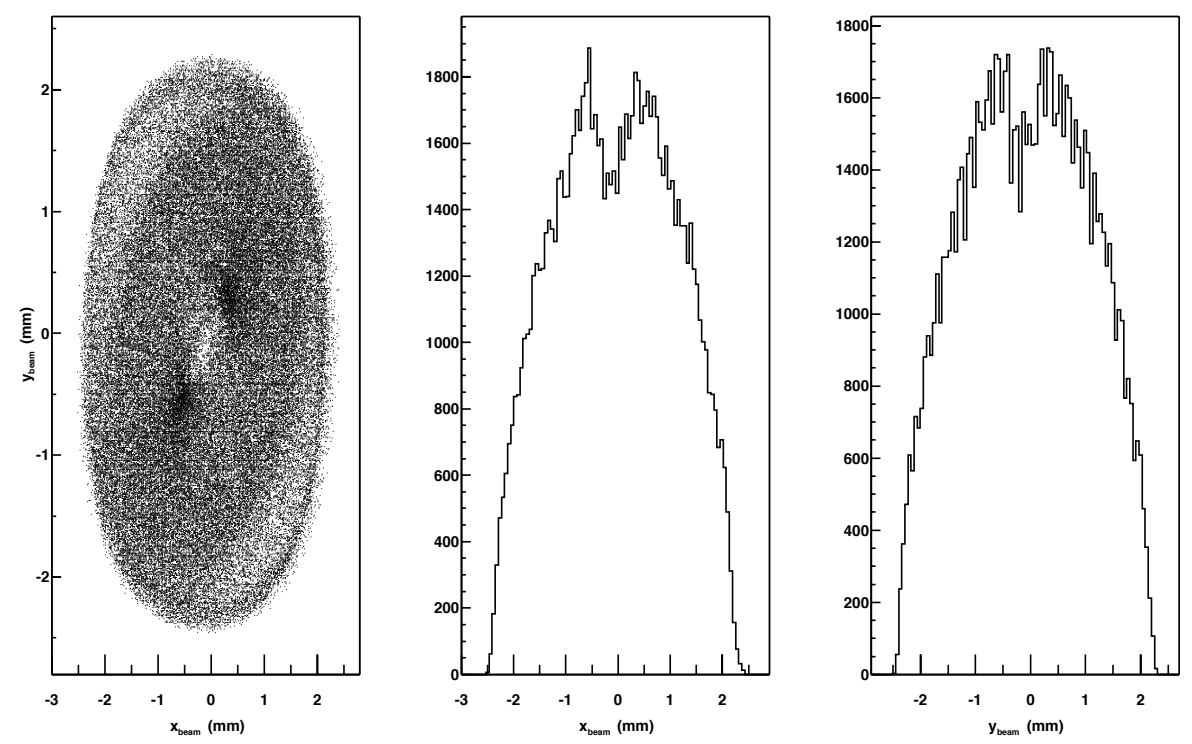

Figure 5.14: The left histogram is a 2-D histogram of the Raster position for a 100000 events. The center histogram is just the $x$ axis distribution for the same 100000 events. The right histogram is the $y$ axis distribution.

\subsubsection{Raster}

Since the beam size from the accelerator is quite small (100 microns), the polarized ${ }^{3} \mathrm{He}$ target used in E97-103 requires constant beam movement or rastering, to avoid cell ruptures as a result of localized heating. The system used in Hall A for E97-103 is a circular raster made by a pair of dipole magnets located $24 \mathrm{~m}$ upstream of the target [41].

The magnets are driven at $18 \mathrm{kHz}$ with a $90^{\circ}$ phase difference between the two so that it makes a circular pattern. The radius of the circular pattern is cycled from $0.2 \mathrm{~mm}$ to $2 \mathrm{~mm}$ at a frequency of $1 \mathrm{kHz}$. Fig. 5.14 shows the raster pattern and the distribution in $x_{\text {beam }}$ and $y_{\text {beam }}$. Fig. 5.15 shows the average beam position in $x_{\text {beam }}$ and $y_{\text {beam }}$ and the raster RMS for the for the first experimental kinematic $\left(Q^{2}=0.54\right.$ $\mathrm{GeV}^{2}$ ). There was a coordinate shift in $y_{\text {beam }}$ because of physical adjustments of the target. 

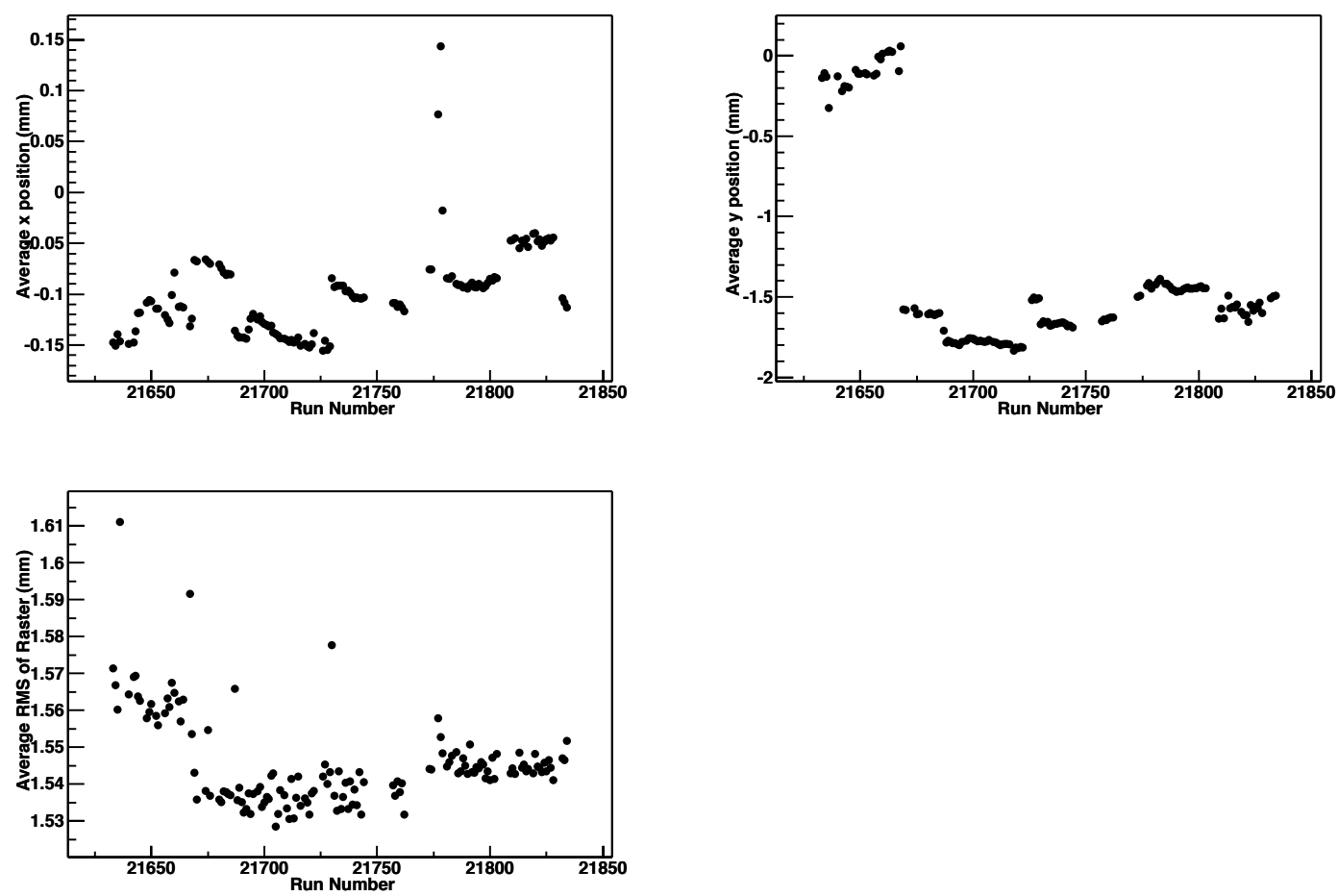

Figure 5.15: The upper-left hand plot is the average $x_{\text {beam }}$ position for the right spectrometer runs for the first kinematic. The upper-right hand plot is the average $y_{\text {beam. The }}$ lower-right hand plot is the average RMS of each run. 


\section{CHAPTER 6 \\ The Polarized ${ }^{3}$ He Target System}

\subsection{Why a Polarized ${ }^{3} \mathrm{He}$ Target?}

The ideal target for measuring neutron spin-structure functions would be a high-density collection of polarized neutrons. Unfortunately, the finite half-life of the neutron $(885.7 \pm 0.8 \mathrm{~s}[49])$ and the difficulty of manipulating neutral particles make a free polarized neutron target impractical for precise spin-structure function measurements. A suitable substitute is a polarizable nuclear target.

Deuterium was used as an effective polarized neutron target in spin-structure function measurements at SLAC (E143 [32], E155 [34] and E155X [50]) and at Jefferson Lab (E93-009,E91-023 and E01-006). These experiments use solids like ${ }^{6} \mathrm{LiH}$ or ${ }^{15} \mathrm{NH}_{3}$ with the hydrogen replaced with deuterium for target material. The target systems uses a process known as dynamic nuclear polarization to achieve a typical deuterium polarization of $22 \%$ [50]. While this type of target has a high neutron density, its polarization deteriorates in electron-beam currents above 100 $\mathrm{nA} \mathrm{[51].} \mathrm{In} \mathrm{addition,} \mathrm{the} 5$ Tesla holding field in this type of target causes serious difficulties due to electron beam deflection when polarized perpendicular to the electron beam.

Polarized ${ }^{3} \mathrm{He}$ has also been used to study spin-structure functions by experiments at SLAC (E142 [38] and E154 [52]), at Jefferson Lab (E94-010,E95-001,E99117 and this experiment) and in the HERMES experiment at DESY [39]. The HERMES experiment used a low-density (areal density of $3.3 \times 10^{14}$ atoms $/ \mathrm{cm}^{2}$ [39]) internal target designed for the HERA storage ring at DESY and not appropriate for 
fixed-target accelerators like SLAC and Jefferson Lab. The SLAC and Jefferson Lab experiments instead have used optically polarized rubidium to polarize ${ }^{3} \mathrm{He}$ gas in sealed glass cells. While this results in a much lower neutron density $\left(2.7 \times 10^{20} \mathrm{~cm}^{3}\right)$, it has the benefits of high average polarization (35-40\%) and maintaining that performance in electron beam currents up to $15 \mu$ Amps [53]. In addition, changing target field direction is simple and the relatively small holding field results in insignificant beam deflection.

Because it could be operated using Jefferson Lab's high electron-beam current and could be easily switched from longitudinal to transverse polarization, the Jefferson Lab polarized ${ }^{3} \mathrm{He}$ target best suited the physics goals of E97-103. The target performed well during E97-103 with a 40\% average polarization and minimal complications.

\subsection{A Quick Word About Other ${ }^{3}$ He Polarization Techniques}

The Jefferson Lab polarized ${ }^{3} \mathrm{He}$ target is based on the transfer of polarization from polarized rubidium vapor to ${ }^{3} \mathrm{He}$ nuclei. It should be noted that this is not the only way to polarize ${ }^{3} \mathrm{He}$. A common technique, known as 'Meta-stability Exchange Optical Pumping', polarizes the ${ }^{3} \mathrm{He}$ nucleus by hyperfine exchange with excited ${ }^{3} \mathrm{He}$ atoms. It can achieve very high polarizations $(\approx 70 \%)$, but unfortunately metastability exchange only occurs efficiently at pressures below 1 atmosphere. Compression techniques, in principle, can be used, but have not been implemented at Jefferson Lab. A reference on applying meta-stable optical pumping to electron scattering physics can be found in [54] and [55].

\section{$\underline{6.3}$ Jefferson Lab Polarized ${ }^{3} \mathrm{He}$ Target Overview}

The Jefferson Lab polarized ${ }^{3} \mathrm{He}$ target, pictured in Fig. 6.1 uses optically polarized rubidium to polarize high-pressure ${ }^{3} \mathrm{He}$ gas sealed in double-chambered glass 


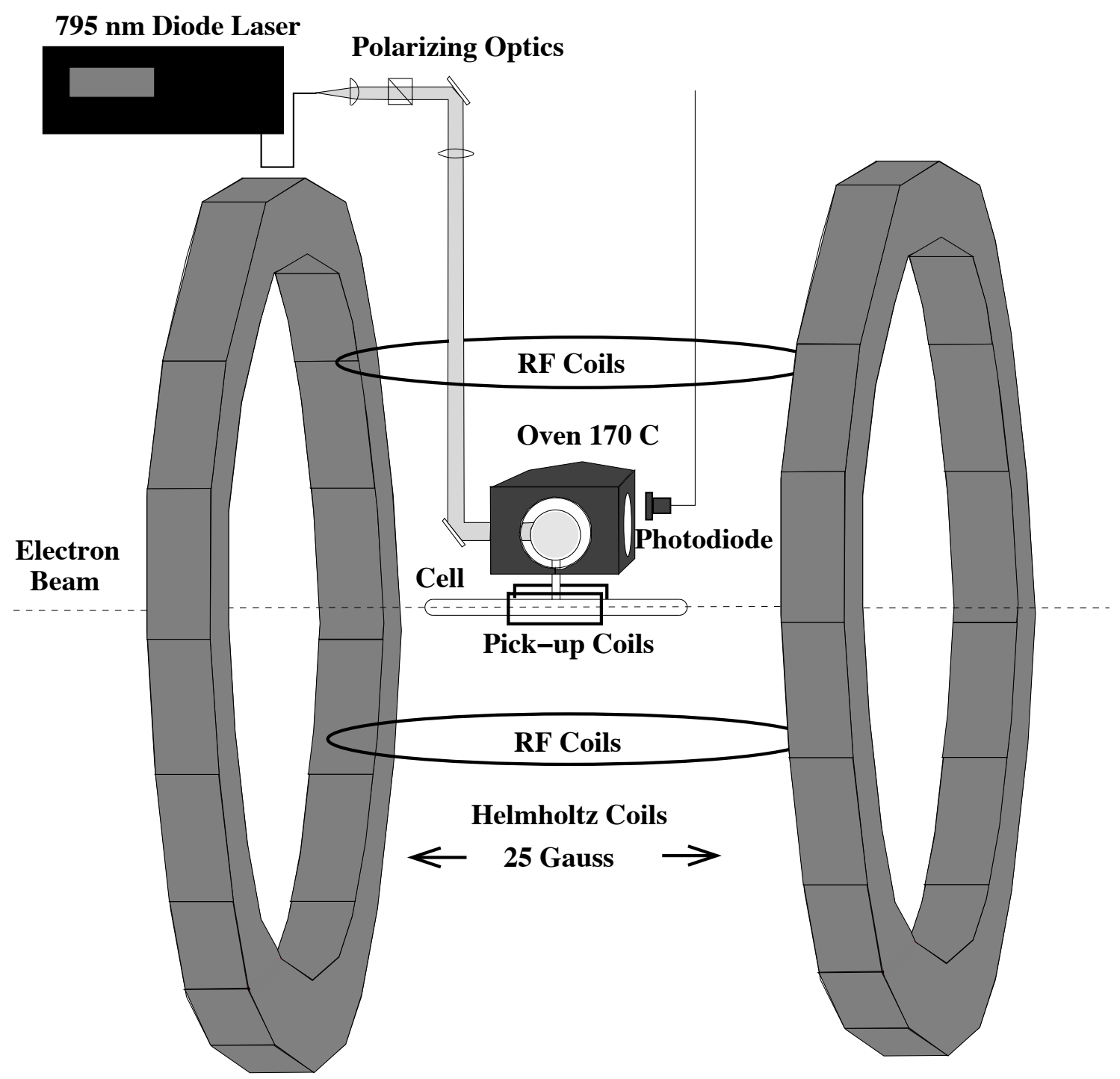

Figure 6.1: A diagram of the polarized ${ }^{3}$ He target system. The transverse Helmholtz coils are not shown for clarity. 
cells. The cells are located in two orthogonal sets of 25 Gauss Helmholtz coils whose combined fields determine the polarization direction. The rubidium is polarized using $795 \mathrm{~nm}$ circularly-polarized light from three $30 \mathrm{~W}$ diode lasers. The polarized rubidium then polarizes the ${ }^{3} \mathrm{He}$ in a spin-exchange process.

The glass cells used to contain the target material have two chambers : a spherical pumping chamber where the optically-polarized rubidium polarizes the ${ }^{3} \mathrm{He}$ nuclei and a cylindrical target chamber where the electron beam scatters off the target material. The pumping chamber is maintained at a temperature of at least $170^{\circ}$ degrees to maintain sufficient rubidium vapor density for optimum spin-exchange. The quality of these cells is fundamental to attaining high ${ }^{3} \mathrm{He}$ polarization.

The polarization of the target material is determined by two independent polarimetry systems. The first uses the signal produced by the nuclear magnetic resonance (NMR) of ${ }^{3} \mathrm{He}$ nuclei to measure the polarization of the ${ }^{3} \mathrm{He}$ in the target chamber. This signal is calibrated by the known NMR signal of water. The second method extracts the polarization of ${ }^{3} \mathrm{He}$ by measuring the change in the electron paramagnetic resonance $(\mathrm{EPR})$ of rubidium when the direction of the polarized ${ }^{3} \mathrm{He}$ atoms is reversed. Using two polarimetry methods reduces the systematic uncertainty in the target polarization.

The Jefferson Lab polarized ${ }^{3}$ He target performed well during E97-103 delivering average polarization above $40 \%$ and requiring no serious maintenance that took away alloted beam time.

\subsection{Polarizing ${ }^{3} \mathrm{He}$ Using Rubidium}

\subsubsection{Polarizing Rubidium}

The energy levels of the atomic spin orbitals of rubidium electrons separate in a magnetic field. Because of this splitting, and because of spin conservation, circularly polarized laser light can excite valence electrons of a specific spin state. 


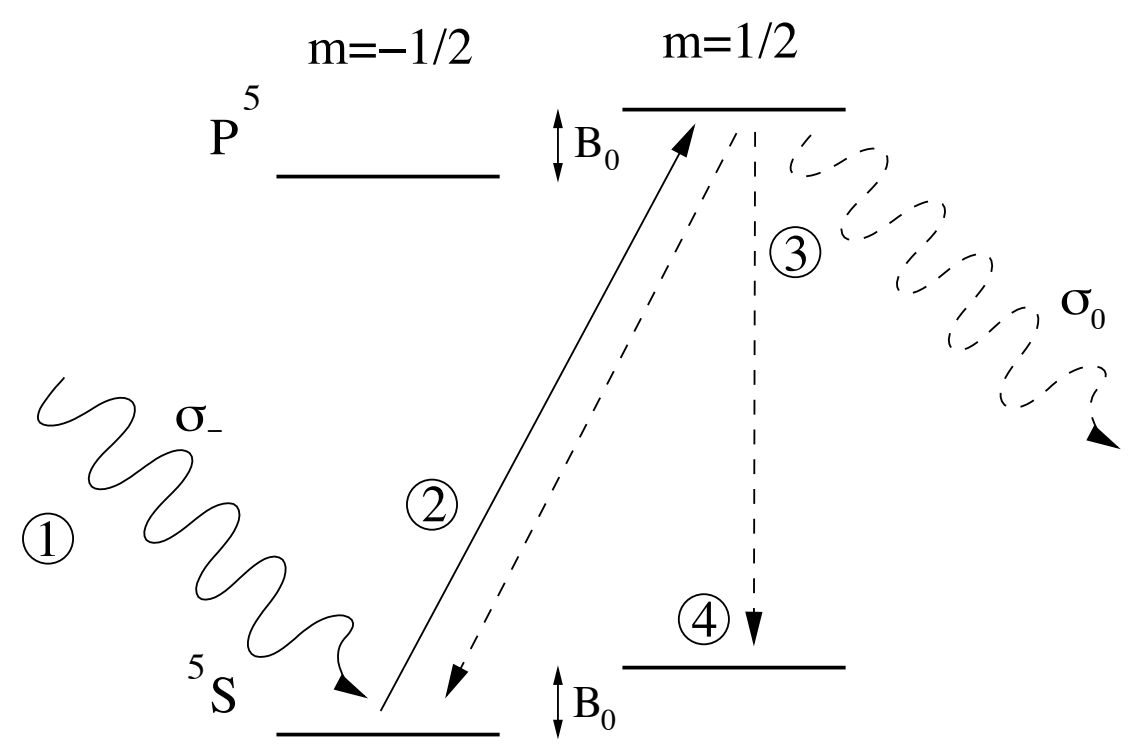

Figure 6.2: A diagram explaining optical pumping. (1) Rb atoms in a magnetic field are exposed to circularly polarized laser light (2) The valence electron is excited from the ${ }^{5} S_{-1 / 2}$ state to the ${ }^{5} P_{1 / 2}$ state (3) The electron decays by emitting a photon into either the ${ }^{5} S_{-1 / 2}$ where it repeats steps (2) and (3) or into the ${ }^{5} S_{1 / 2}$ state where (4) it remains.

Left circularly polarized $795 \mathrm{~nm}$ photons excite electrons of the $\mathrm{m}=1 / 2{ }^{5} \mathrm{~S}$ state to the $\mathrm{m}=-1 / 2{ }^{5} \mathrm{P}$ state, while right circularly polarized light of the same wavelength excites electrons in the $\mathrm{m}=-1 / 2{ }^{5} \mathrm{~S}$ state to the $\mathrm{m}=1 / 2{ }^{5} \mathrm{P}$ state.

The excited electron will decay, by emitting a photon, into either the $m=1 / 2$ or $\mathrm{m}=-1 / 2{ }^{5} \mathrm{~S}$ state. Since the light is only exciting the electrons from one of these $\mathrm{m}$ states, all the valence electrons of the exposed rubidium atoms will eventually occupy the opposite spin orbital. This process is commonly known as "optical pumping" and results in polarized rubidium atoms. A diagram explanation of optical pumping is shown in Fig. 6.2.

The emitted photon from the electron decays is unpolarized and can be reabsorbed by other rubidium atoms. This process makes high rubidium polarization impossible. Fortunately, this effect can be reduced by introducing nitrogen into the system. Nitrogen can absorb light at the emitted frequencies and since the light is absorbed into the molecule's vibrational and rotational motion it doesn't emit 
another photon.

The average polarization of rubidium vapor can be expressed by the equation:

$$
P_{\mathrm{Rb}}=\frac{R}{R+\Gamma_{\mathrm{SD}}}
$$

where $\Gamma_{\mathrm{SD}}$ is the spin-destruction rate of the rubidium vapor and $\mathrm{R}$ is defined by the equation:

$$
R=\int \Phi(\nu) \sigma(\nu) d \nu
$$

where $\Phi(\nu)$ is the photon flux per unit frequency from the laser light and $\sigma(\nu)$ is the light absorption cross-section. It is obvious from the above equations that the key factors to high rubidium polarization are the laser flux, $\Phi$, and the spin-destruction rate $\Gamma_{\mathrm{SD}}$. The laser flux is simply a function of laser power at the absorption frequency and can be increased as needed. The spin-destruction rate is determined by several factors which are worth exploring in detail.

The spin-destruction rate of rubidium is dominated by rubidium transferring spin angular momentum to the rotational angular momentum of other atoms. In the E97-103 system, the rubidium collisions of significant concern are : Rb-Rb, Rb${ }^{3} \mathrm{He}$ and $\mathrm{Rb}-\mathrm{N}_{2}$. (Collisions with the glass walls of the system are in principle a concern, but because of the high ${ }^{3} \mathrm{He}$ pressure in the E97-103 cells, the diffusion rate of rubidium amongst high-pressure ${ }^{3} \mathrm{He}$ is small. According to Wagshul and Chupp [56], only rubidium within $\approx 0.1 \mathrm{~mm}$ of the cell walls are affected by this spin-destruction process.) The total spin-destruction rate from these process can be written:

$$
\Gamma_{\mathrm{SD}}=k_{\mathrm{Rb}-\mathrm{He}}\left[{ }^{3} \mathrm{He}\right]+k_{\mathrm{Rb}-\mathrm{N}_{2}}\left[\mathrm{~N}_{2}\right]+k_{\mathrm{Rb}-\mathrm{Rb}}[\mathrm{Rb}]
$$

where $k_{\mathrm{Rb}-\mathrm{i}}$ is the spin-destruction rate constant for collision $\mathrm{Rb}-\mathrm{i}$. The constants for these have been calculated by Wagshul and Chupp [56]:

$$
k_{\mathrm{Rb}-\mathrm{Rb}}=8 \times 10^{-13} \mathrm{~cm}^{3} / \mathrm{s}
$$




$$
\begin{aligned}
k_{\mathrm{Rb}-{ }^{3} \mathrm{He}} & \leq 2 \times 10^{-18} \mathrm{~cm}^{3} / \mathrm{s} \\
k_{\mathrm{Rb}-\mathrm{N}_{2}} & =8 \times 10^{-13} \mathrm{~cm}^{3} / \mathrm{s}
\end{aligned}
$$

These numbers have a strong temperature dependence and should be treated as factor of "2" estimates [57].

For the E97-103 experiment, the approximate densities of the components $\left(\left[{ }^{3} \mathrm{He}\right]=2 \times 10^{20} \mathrm{~cm}^{-3},\left[\mathrm{~N}_{2}\right]=1.8 \times 10^{18} \mathrm{~cm}^{-3},[\mathrm{Rb}]=4 \times 10^{14} \mathrm{~cm}^{-3}\right)$ gives the following [57]:

$$
\Gamma_{\mathrm{SD}}=400_{\mathrm{Rb}-{ }^{3} \mathrm{He}}+14_{\mathrm{Rb}-\mathrm{N}_{2}}+320_{\mathrm{Rb}-\mathrm{Rb}}=734 \mathrm{~s}^{-1}
$$

One can see from this result that the $\mathrm{Rb}^{-}{ }^{3} \mathrm{He}$ and $\mathrm{Rb}-\mathrm{Rb}$ collision rates are similar. It should be noted that Rb-Rb collisions does not depolarize the rubidium sample since spin is conserved in the collision process. It should also be noted that the $\mathrm{Rb}-{ }^{3} \mathrm{He}$ collisions mentioned here absorb the rubidium spin into their angular momentum and are not the spin exchange collisions with the ${ }^{3} \mathrm{He}$ nuclei which are needed for polarized ${ }^{3} \mathrm{He}$.

\subsubsection{Polarizing ${ }^{3} \mathrm{He}$ With Polarized Rubidium}

Rubidium can transfer its electron polarization to the nucleus of a ${ }^{3} \mathrm{He}$ atom through a hyperfine-like interaction. This spin-exchange process between Rb and ${ }^{3} \mathrm{He}$ has a smaller contribution $\left(\Gamma_{\mathrm{SE}}=24 \mathrm{~s}^{-1}\right)$ to $\Gamma_{S D}$; consequently, only approximately $3 \%$ of polarized rubidium atoms lose their polarization through a spinexchange process with ${ }^{3} \mathrm{He}$. This makes the polarization of ${ }^{3} \mathrm{He}$ through rubidium spin-exchange an inherently inefficient process.

The polarization of ${ }^{3} \mathrm{He}$ with respect to time can be described by:

$$
P_{{ }^{\mathrm{He}}}(t)=\left\langle P_{\mathrm{Rb}}\right\rangle \frac{\gamma_{\mathrm{SE}}}{\gamma_{\mathrm{SE}}+\Gamma}\left\{1-\exp \left[-\left(\gamma_{\mathrm{SE}}+\Gamma\right) t\right]\right\}
$$

where $\gamma_{\mathrm{SE}}=k_{\mathrm{SE}}[\mathrm{Rb}], \Gamma$ is the ${ }^{3} \mathrm{He}$ polarization destruction rate and $\left\langle P_{\mathrm{Rb}}\right\rangle$ is the average rubidium polarization in the system. 
$\Gamma$ is the quantity which best describes the level of ${ }^{3} \mathrm{He}$ polarization a system will achieve. This quantity is can be broken into its four most important sources of depolarization:

$$
\frac{1}{\Gamma_{3_{\mathrm{He}}}}=\frac{1}{\Gamma_{\text {Dipole }}}+\frac{1}{\Gamma_{\text {Wall }}}+\frac{1}{\Gamma_{\nabla B}}+\frac{1}{\Gamma_{\text {BeamDepol. }}}
$$

where $\Gamma_{\text {Dipole }}$ is depolarization from ${ }^{3} \mathrm{He}-{ }^{3} \mathrm{He}$ collisions, $\Gamma_{\text {Wall }}$ is depolarization from interactions with the glass walls, $\Gamma_{\nabla B}$ is depolarization from magnetic field gradients and $\Gamma_{\text {BeamDepol. }}$ is depolarization by the electron beam.

$\Gamma_{\text {Dipole }}$ is the dominant factor in high-density ${ }^{3} \mathrm{He}$ system like the one used in E97-103. It is a result of spin-destructive ${ }^{3} \mathrm{He}-{ }^{3} \mathrm{He}$ collisions. This term can be expressed by:

$$
\Gamma_{D}=\frac{[3 \mathrm{He}]}{744} \mathrm{hrs}^{-1}
$$

where $\left[{ }^{3} \mathrm{He}\right]$ is in amagats [58].

Another important process in polarization relaxation is ${ }^{3} \mathrm{He}$ interactions with the glass cell walls. There are multiple reasons that the cell walls cause depolarization. One is the out-gassing paramagnetic gases like $\mathrm{O}_{2}$ or $\mathrm{NO}$ from the walls when the cell is heated. Another is paramagnetic material like $\mathrm{RbO}_{2}$ on the surface of the cell walls. A third reason is increased sticking time of ${ }^{3} \mathrm{He}$ to the surface of the walls due to microscopic fissures in the glass. It is uncertain which effect is the dominant source of spin-destruction. $\Gamma_{\text {Wall }}$ varies significantly from cell to cell, depending on the conditions of its manufacture. A good cell can have a $\Gamma_{\text {Wall }}<1 / 200 \mathrm{hrs}^{-1}$. A bad cell can have a $\Gamma_{\text {Wall }}>1 \mathrm{hrs}^{-1}$.

Magnetic field gradients can also contribute to depolarization. The relevant formula is:

$$
\Gamma_{\nabla \mathrm{B}}=D_{3} \mathrm{He} \frac{\left|\nabla B_{x}\right|^{2}+\left|\nabla B_{y}\right|^{2}}{B_{z}^{2}}
$$

where $D_{3 \mathrm{He}}$ is the self-diffusion constant of ${ }^{3} \mathrm{He}$ and $B_{z}$ is the magnitude of the holding field. $\Delta B_{x}$ and $\Delta B_{y}$ are the field gradients perpendicular to the holding 
field. The self-diffusion constant in the case of a high-density system is small $\left(D_{3} \mathrm{He}=\right.$ $0.28 \mathrm{~cm}^{2} / \mathrm{s}$. The gradient in the Jefferson Lab Polarized ${ }^{3}$ He system is usually kept below $10 \mathrm{mGauss} / \mathrm{cm}$ which translates to a negligible $\Gamma_{\nabla \mathrm{B}}=6000 \mathrm{hr}^{-1}$.

The target, of course, is subjected to a high-current electron beam. The electrons from this beam ionize ${ }^{3} \mathrm{He}$ atoms. The single electron from the ionized ${ }^{3} \mathrm{He}$ can depolarize the nucleus by hyperfine interaction with the nucleus. ${ }^{3} \mathrm{He}+$ ions can also form ${ }^{3} \mathrm{He}_{2}$ molecules which can depolarize the nucleus by spin-rotation interaction; however, this is a much less frequent process since the molecule is quickly broken up by collisions with ${ }^{3} \mathrm{He}$ and $\mathrm{N}_{2}$. As described in [59] and [60], the beam depolarization can be be written :

$$
\Gamma_{\text {BeamDepol. }}=\left(n_{\mathrm{a}}+n_{\mathrm{m}}\right) \Gamma_{i}
$$

where $n_{\mathrm{a}}+n_{\mathrm{m}}$ is the number of ${ }^{3} \mathrm{He}$ atoms depolarized over the number ionized, where $n_{\mathrm{a}}$ is the contribution from ionized atoms and $n_{\mathrm{m}}$ is the contribution from ionized ${ }^{3} \mathrm{He}_{2}$ molecules. This fraction can range from close to zero to many thousands. $\Gamma_{i}$ is the mean ionization rate per atom and is defined by:

$$
\Gamma_{\mathrm{i}}=i_{\mathrm{b}}\left(\frac{\mathrm{d} E}{\mathrm{~d} x}\right)^{3} \mathrm{He} \frac{L}{E_{\mathrm{ion}}^{3} \mathrm{He} V}
$$

where $i_{\mathrm{b}}$ is the beam current in particles per second, $(\mathrm{d} E / \mathrm{d} x)^{3} H e$ is the energy loss per incident electron per ${ }^{3} \mathrm{He}$ atom per area, $E_{\mathrm{ion}}^{3} \mathrm{He}$ is the mean energy required to produce an ion pair from a target atom, $L$ is the target length and $V$ is the target volume. According to Bonin et al. [60] $n_{m}$ is suppressed by the presence of $\mathrm{N}_{2}$ and can be neglected.

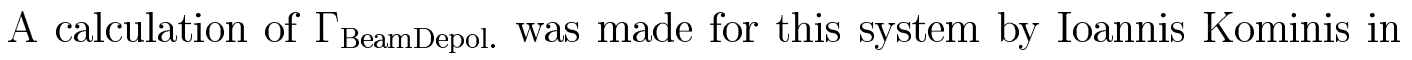
his dissertation [61]. His calculation was based on the formalism discussed above. The predicted value for $\Gamma_{\text {BeamDepol. was }} \leq 1 / 30$ hrs for a current of $10 \mu$ Amps. The error on this measurement is difficult to determine. The error bars presented on 


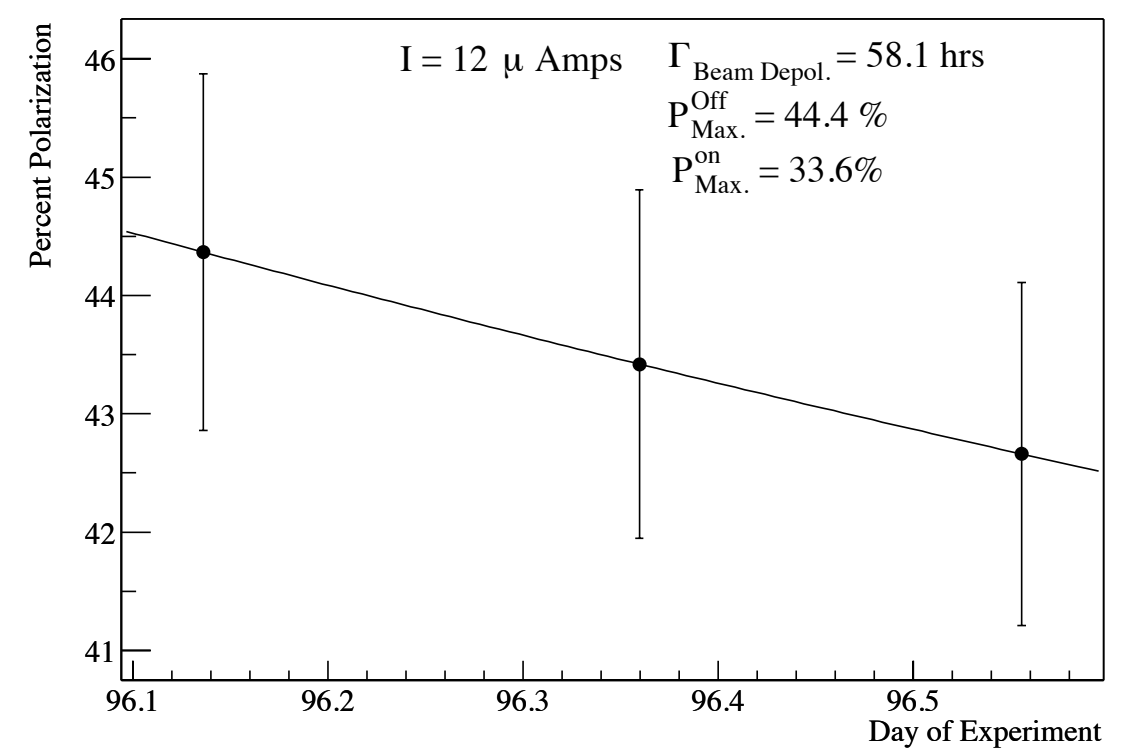

Figure 6.3: The effect of the electron beam on the polarization of a polarized ${ }^{3}$ He target.

the polarization are mostly systematic errors from the calibration. The individual signals have error bars of $0.5 \%$.

The effect of beam depolarization on the ${ }^{3} \mathrm{He}$ target can be measured empirically. During an experiment, it is complicated to get a good data set because for the most accurate measurement, the target must be at its highest polarization, the beam must be at nearly constant current for a long period of time and the laser power on the pumping chamber must be constant. Unfortunately, these three conditions are rarely met simultaneously. The target rarely gets to its highest polarization due to time constraints. The beam rarely goes through long periods of constant current without being off on the order of an hour or two. In E97-103, the constant configuration changes between longitudinal and transverse and the unequal laser power of the two, made extraction of this quantity problematic. However, it was extracted for a short period during the ${ }^{3} \mathrm{He}$ elastic commissioning using the formula:

$$
P(t)=\left(P_{\mathrm{Max}}^{\mathrm{off}}-P_{\mathrm{Max}}^{\text {on }}\right) e^{-\Gamma_{\mathrm{BeamDepol}} . t}+P_{\mathrm{Max}}^{\mathrm{on}}
$$




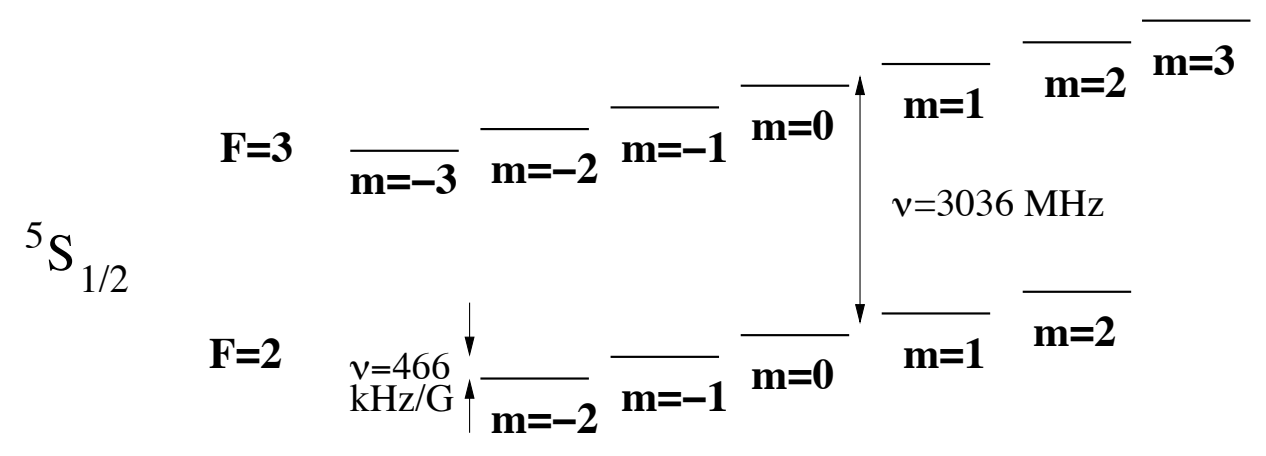

Figure 6.4: The electron states of the ${ }^{5} S_{1 / 2}$ orbital of rubidium.

where $P_{\text {Max }}^{\mathrm{off}}$ is the maximum polarization with the electron beam off and $P_{\mathrm{Max}}^{\mathrm{on}}$ is the maximum polarization with the electron beam on. For the data set presented in Fig. 6.3 the $\Gamma_{\text {BeamDepol. }}=58.1 \mathrm{hrs}$ with a signifcant amount of uncertainty from 29 hrs to 100 hrs.

\subsubsection{Hyperfine Splitting from the Nucleus}

While optical polarization of rubidium can be explained using simple Zeeman splitting of the electron states, for explanations of more complicated phenomena such as collisional mixing and electron-paramagnetic resonances (EPR) one must pay attention to the additional level splitting caused by hyperfine interaction spin coupling. In general, the eigenstates of the total angular momentum projected on the axis of the magnetic field can be represented by the equation:

$$
F_{z}=I_{z}+S_{z}
$$

where $F_{z}$ is the total angular momentum along the holding field direction, $I_{z}$ is the spin of the rubidium nucleus and $S_{z}$ is the of the electron. $F_{z}$ determines the energy level of the electron state.

Because the spin of ${ }^{85} \mathrm{Rb}$ nucleus is $5 / 2\left({ }^{87} \mathrm{Rb}\right.$ has $\left.\mathrm{I}=3 / 2\right)$, the eigenvalue $m_{I}$ goes from $-5 / 2$, to $5 / 2$ by increments of $1(-5 / 2,-3 / 2,-1 / 2$, etc.). The eigenvalue corresponding to the spin, $m_{S}$, of the electron can either be $1 / 2$ or $-1 / 2$. When not in a 
magnetic field the valence electron has two states $F=2$ or $F=3$, corresponding to when the electron's angular momentum is parallel or anti-parallel to nuclei. However, when the atom is in a magnetic field, a direction preference emerges and the different nuclear spins becomes distinguishable. In a magnetic field, the $F_{z}$ states split into $2 F+1$ levels as shown in Fig. 6.4 corresponding to different combinations of nucleus and valence electron spin states.

A phenomena associated with these nucleus-electron hyperfine spin states is collisional mixing. Because of spin-conserved $\mathrm{Rb}-\mathrm{Rb}$ collisions, electrons in ${ }^{5} \mathrm{P}$ states or ${ }^{5} \mathrm{~S}$ states are distributed in their hyperfine nucleus state. Because of this distribution electrons will have different excitation and photon emission energies. Collisional mixing the photons emitted from excitation of the electron paramagnetic resonance will have a broad range of frequencies rather than just one. This allows separation of these photons from the intense background of the laser light.

\subsection{Polarized ${ }^{3} \mathrm{He}$ Cell Construction}

\subsubsection{The Fabrication of the Glass Target Cells}

The use of glass cells for polarized ${ }^{3} \mathrm{He}$ targets began in the late 1980's with the form factor experiments at the MIT-Bates laboratory [54]. The technology was transfered first to SLAC for the E142 [38] and E154 [40] and then to Jefferson Lab for experiments E94-010 [62] and E95-001 [63]. Consequently, by the time of E97103, a set of techniques for creating high-quality glass cells for polarizing ${ }^{3} \mathrm{He}$ had been established.

The procedures for cell construction, based on the experience of these past experiments, focus on two factors that affect the quality of the cell : the presence of paramagnetic materials on the cell walls and the smoothness of the interior walls. Paramagnetic materials are mostly eliminated by starting with clean glass, avoiding processes that could add paramagnetic material and baking the cell extensively 


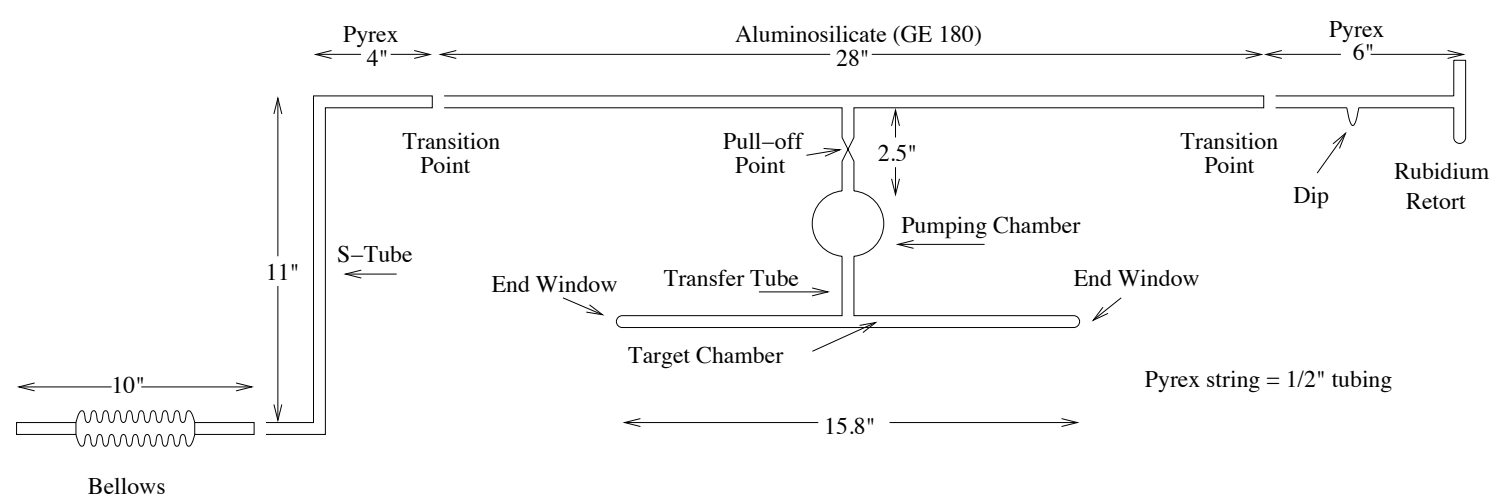

Figure 6.5: Diagram of the glass cell assembly created by the glass-blower.

before filling it. The smoothness of the cell walls is largely depends on techniques used by the glass-blower when constructing the cell. Since both of these qualities are difficult to monitor during the cell-making process, there is still considerable variation in the quality of the cells.

Because of these demands, cell construction is done by a professional glass worker. Mike Souza at Princeton, who did the pioneering work for the SLAC experiments, and William Shoup at the University of Virginia did the glass blowing for this experiment. The glass blower constructs the cell and a glass assembly (or string) as shown in Fig. 6.5. The cell is made of a spherical pumping chamber and a cylindrical target chamber. These chambers are connected by a short transfer tube. The ends of the target chamber are capped by thin walled end-windows. The glass assembly serves two purposes : to connect the cell to the gas filling system and to connect the retort where the rubidium enters the system. The cell is then sent to a lab where it is filled with the requisite amounts of ${ }^{3} \mathrm{He}, \mathrm{N}_{2}$ and rubidium.

The cells start off as 1/2" GE 180 aluminosilicate glass tubing. Aluminosilicate glass is chosen because it holds up well in a radiation environment, contains very few magnetic or paramagnetic compounds and sufficiently low porosity to high-pressure ${ }^{3} \mathrm{He}$ without leaking. In previous experiments the cells had been made of Dow Corning 1720 aluminosilicate glass. GE 180 was used because it is easier material 
to work with and because of the limited supply of Dow Corning 1720. However, the trade off in choosing GE 180 is that it has a shorter radiation length than Dow Corning 1720.

The stock tubing acquired from the manufacturer is cleaned with methanol. The tubing is resized using a surface mix torch burning methane and oxygen and a glass-working lathe [64]. The outside diameter of the tubing is then adjusted using a fixed graphite block. This resizing of the tubing is done not only to match the dimensional specifications of the target cell, but also seems to help with getting rid of paramagnetic materials and generally improves the surface.

The pumping chamber is shaped to specifications by heating a sealed end of a piece of tubing then blowing it into a sphere. An another piece of tubing is stuck into the still molten glass so that the pumping chamber can be connected to the rest of the assembly. Once cool, the pumping chamber is pressure tested to $300 \mathrm{psi}$ for 20 minutes [64].

There are rather strict requirements for the end windows of the target chamber. The windows must be $100 \pm 20$ microns thick. This is to reduce energy loss by the electron beam and to reduce background scattering into the detector acceptance. The glass blower makes a series of these end windows. He then uses a digital indicator (Mitutoyo digimatic) with a resolution of 2 micron to see if the windows match specifications [64]. A good end window will then be attached to the end of the target chamber. The end window thickness is re-measured again using a laser system after the cell is filled.

Once the cell is assembled, it is annealed in a $785^{\circ} \mathrm{C}$ oven for 10 minutes. This relieves any stress in the glass that may have been formed during its construction. Once annealed, the cell is attached to the rest of the assembly. The rubidium retort and the S-tube piece shown in Fig. 6.5 are constructed out of Pyrex. The S-tube connects the cell assembly to the cell-filling system through a bellows tube. The 
bellows tube is flexible and allows adjustments to be made to the cell position when attached. The bellows are also crucial for removing the cell assembly from the cell during the pull-off procedure. The rubidium retort consists of an open-ended cylinder where a rubidium ampoule is inserted and a dip where the rubidium is moved to during the filling procedure. Both the rubidium retort and the S-tube are connected to the cell using a transition glass of Corning 3320 since GE180 and Pyrex can't be connected directly. The bellows are also connected with a glass-to-metal transition.

Once the cell is assembled it is transported to a filling lab. Cork or rubber stoppers are put in the open ends of the glass assembly to keep the interior clean. Sometimes the assembly is filled with argon gas for additional cleanliness.

\subsubsection{The Cell Filling System}

Once the cell is constructed it must be filled with the target material, plus the rubidium and nitrogen required to polarize the ${ }^{3} \mathrm{He}$. This is done by a gas filling system specifically designed for polarized ${ }^{3} \mathrm{He}$ cells. The procedures to fill the cell are as important to creating a good cell as the glass construction.

The filling system shown in Fig. 6.6 consists of several systems. The first is a set of pumps that create a vacuum throughout the system and the cell assembly. There is a roughing pumping used when the pressure is above $1 \times 10^{-3}$ Torr. The turbo pump can be used when the pressure is below $1 \times 10^{-1}$ Torr and can pump down to pressures in the $1 \times 10^{-8}$ to $1 \times 10^{-9}$ Torr range. There is also an ion pump which is used to maintain good vacuum near the lecture bottles.

The next part of the cell filling system are the lecture bottles and their regulators. The lecture bottles are bought from Spectra Gases and contain 99.99\% pure ${ }^{3} \mathrm{He}$ and nitrogen gas. Each bottle contains $25 \mathrm{~L}$. The ${ }^{3} \mathrm{He}$ is very expensive $(\approx \$ 100$ a liter) and considerable effort is made to conserve it. Spectra Gases attached valve 


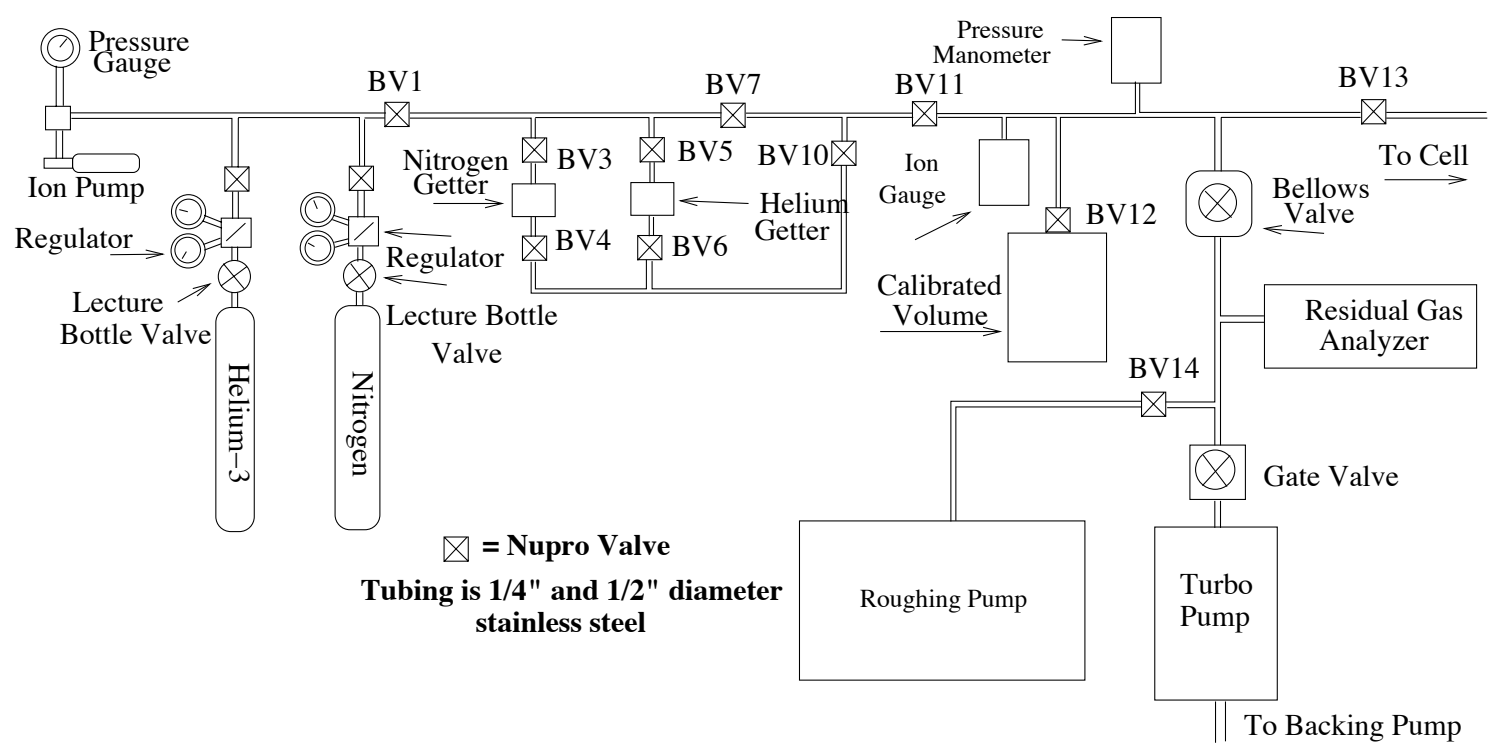

Figure 6.6: Cell Filling system

stems to the bottles which can then be attached to regulators which control the flow of gas from the bottles.

In addition to those devices, there are several smaller sections. There are two heated getters, one for helium and one for nitrogen. These getters act as filters, absorbing impurities into materials inside the getter and letting the desired gas to flow through. The ion gauge is used to measure pressure below $1 \times 10^{-3}$ Torr. A pressure manometer is used for pressures above that. The calibrated volume is used to measure gas in the cell filling process. The residual gas analyzer is used for diagnostics when cleaning the system, but is turned off when a cell is attached to the system.

\subsubsection{Preparing for Cell Filling}

The first step of the cell filling procedure is to attach the glass assembly to the cell filling system pictured in Fig. 6.6. The bellows on the cell assembly have a Swagelok fitting on it that can be attached to the filling system. The cell assembly is supported by aluminum rods held together by clamps. 


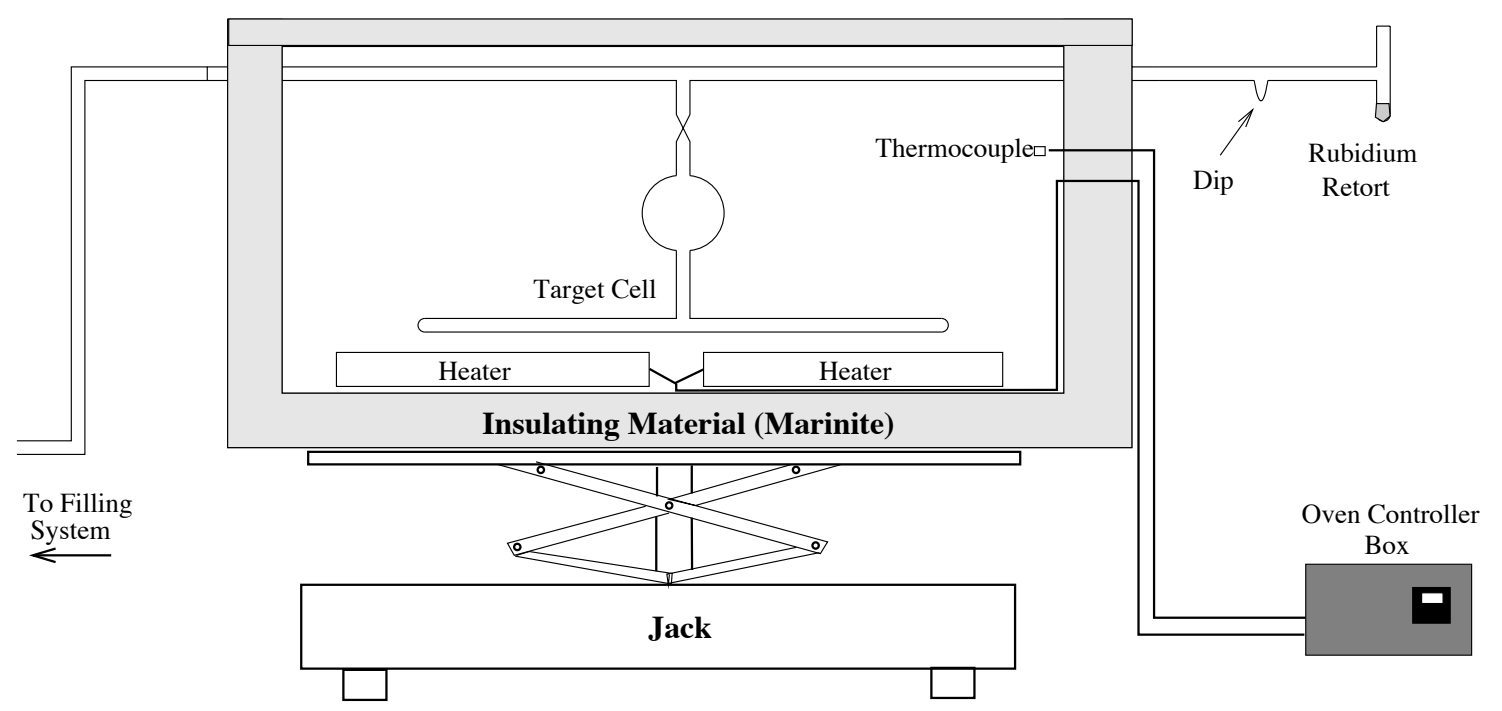

Figure 6.7: Cell Baking Configuration

The next step is to insert the rubidium into the cell assembly. Rubidium is highly reactive with air and comes sealed in an ampoule. The retort on the cell assembly shown in Fig. 6.5 is a cylindrical tube that is open at the top. Before inserting the rubidium, BV11 (a Nupro Valve), BV12 and the gate valve in the filling system are closed. The valve BV14 and the roughing pump is turned on to pump on the cell assembly. The rubidium ampoule is opened and both the rubidium and the ampoule are inserted into the retort. The open end of the retort is sealed by a methane/oxygen torch. The system will then be rapidly evacuated by the roughing pump.

At this point, the cell and the cell assembly need to baked at high temperature to remove any impurities on the surface. The filling system and cell assembly are first evacuated by the turbo pump. This will get the pressure down to $1 \times 10^{-7}$ Torr. The cell is then placed in an oven as shown in Fig. 6.7. The oven is made of marinite which is a type of calcium silicate board. It is light weight and a good insulator. The heat of the oven is generated by two sets of heating elements that sit at the bottom. They are controlled by an Omega autotune controller that uses 
a thermocouple in the oven to read the temperature. The oven is heated to $420 \mathrm{C}$ and that temperature is maintained for three or four days.

It is important when baking the cell, to occasionally heat the rest of the assembly that is not in the oven. This is done with a methane/oxygen torch with a cooler, bushy flame to avoid melting the glass assembly (flamebaking). The procedure is to heat the S-tube portion and the rubidium retort except for where the rubidium is, two to three times a day for about 10 minutes each time. The rubidium will be heated later in the procedure when the cell is cleaner. Generally, when this is done the pressure in the system will rise to $1 \times 10^{-6}$ Torr temporarily, but will soon fall to an even lower pressure than before the heating. This procedure is repeated until the vacuum is consistently on the order of $10^{-9}$ Torr or below.

Usually after the second day of baking the cell the rubidium is moved from the bottom of the retort to a dip, shown in Fig. 6.7 farther into the cell assembly. The purpose of this is to move rubidium to place where the glass has been heated thoroughly from a place of questionable cleanliness. Also, mild heating of the rubidium helps remove gas impurities that may have been in the ampoule at the time of sealing. Rubidium vaporizes easily when heated by a methane/oxygen flame. The key to moving rubidium from one place to another is keeping the part where the rubidium needs to go cool, and heating everything else. A cooler flame seems to keep the rubidium from forming impurities by reacting with the glass. After the rubidium is moved to the dip, the retort is removed from the glass assembly with a torch.

Once the baking process has stopped improving, the oven is turned off and the turbo pump is left on for a period of time to achieve the best vacuum possible. The oven takes a long time to cool, so generally the oven is turned off the night before the cell fill. The vacuum system can get down to pressures $<5 \times 10^{-9}$ Torr. The oven is then removed from around the cell. The getters must be turned on at this 
point so they will be at operating temperature when the cell is filled.

The next step is to move the rubidium from the dip to the cell. This must be done with extreme care since the quality of the cell is strongly affected by this process. The torch should be hotter than when flamebaking, but should not be so hot to melt the glass. Again, the key to moving rubidium in the cell assembly is to leave the area where the rubidium should collect cool, and heat everything else. Making sure the rubidium is vaporized repeatedly during this process helps cleans the rubidium of impurities. This process continues until the rubidium that collects on the pumping chamber walls becomes visible. There does not need to be a lot of rubidium in the pumping chamber. The rubidium shoud be shiny, indicating a general lack of impurities. Oxidized rubidium is paramagnetic and appears black usually indicating a bad cell.

\subsubsection{Measurement of the Cell Assembly Volume}

Once the rubidium is in place, a procedure to measure the cell string volume is performed. The cell filling process requires a reasonably accurate value for the volume of the string, which is the volume of the cell assembly minus the volume of the cell. This can be done because the cell volume can be estimated to reasonable accuracy using only its external dimensions.

The calibrated volume is first filled with a known pressure (referred to as $P_{1}$ here) of nitrogen. The volume of the calibrated volume $\left(V_{C V}\right)$ is known very precisely $(1.064 \pm 0.001 \mathrm{~L})$ from mechanical measurements. This volume of gas is then released first into the small area, referred to as the manifold, between the calibrated volume and the valve to the cell (BV13) with the bellows and BV11 valves closed. The pressure is read $\left(P_{2}\right)$ and then the valve to the cell assembly is opened. The pressure is then read again $\left(P_{3}\right)$.

The ideal gas law can be used to calculate the volume of the cell assembly 


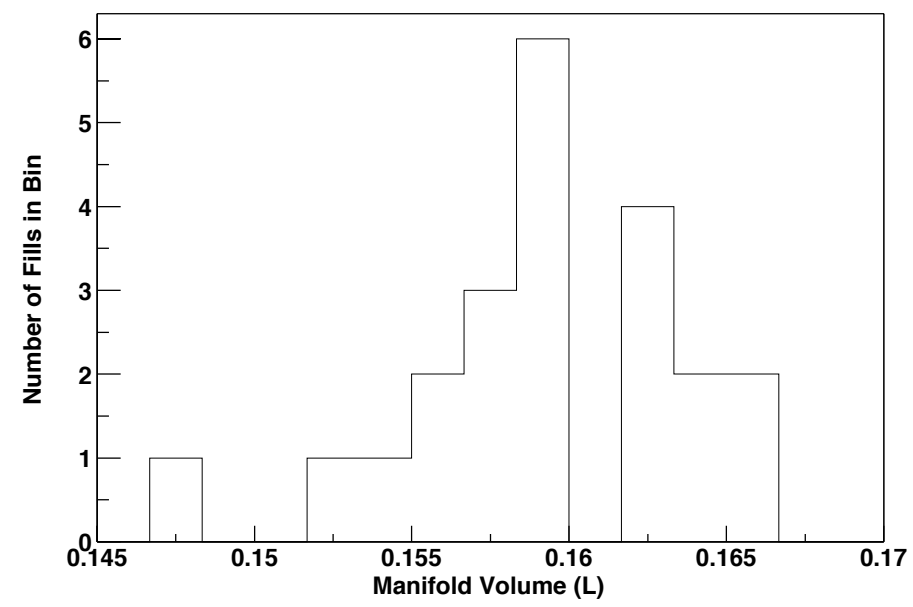

Figure 6.8: A plot of the manifold volume measured during the cell filling process.

with the results of this procedure. If you assume all the entire system is at room temperature then:

$$
\begin{aligned}
V_{\mathrm{M}} & =\frac{P_{1}-P_{2}}{P_{2}} V_{\mathrm{CV}} \\
V_{\mathrm{S}}+V_{\mathrm{C}} & =\frac{\left(P_{1}-P_{3}\right) V_{\mathrm{CV}}-P_{3} V_{\mathrm{M}}}{P_{3}}
\end{aligned}
$$

where $P_{1}, P_{2}$ and $P_{3}$ are the pressures at various steps in the procedure, $V_{\mathrm{CV}}$ is the volume of the calibrated volume, $V_{\mathrm{M}}$ is the volume of the manifold section, $V_{C}$ is the volume of the cell and $V_{\mathrm{S}}$ is the volume of the rest of the cell assembly, referred to as the string. The $V_{\mathrm{C}}$ and $V_{\mathrm{S}}$ can't be separated by this procedure, but $V_{\mathrm{C}}$ can be estimated accurately using external measurements of the cell and then $V_{\mathrm{S}}$ can be extracted from the results of this procedure.

A useful piece of information in estimating the error from this procedure is to look at the consistency of the measurement of the volume of the manifold section. This data is plotted in Fig. 6.8. The data was taken for fills where the manifold volume was the same (the tubing and valve sections were used each time). This procedure is done before and after a cell fill and it seems that the volume measurements done after the cell fills are systematically larger. This is likely due to temperature 
changes from nearby cryogens used in the filling procedure. The overall error is estimated to be $\pm 5 \mathrm{ml}$.

\subsubsection{Filling the Cell with Nitrogen and ${ }^{3} \mathrm{He}$}

Nitrogen, necessary for absorbing unpolarized photons from optically polarized rubidium, is put into the cell at room temperature. The procedure is to fill the manifold with nitrogen that has been purified by the nitrogen getter. The pressure in the manifold is required to get 70 Torr of nitrogen (a reasonable amount) in the final cell can be calculated using:

$$
P_{\mathrm{N}_{2}}=(65 \text { torr }) \frac{V_{\mathrm{C}}\left(V_{\mathrm{M}}+V_{\mathrm{S}}+V_{\mathrm{C}}\right)}{V_{M}\left(V_{S}+V_{C}\right)} .
$$

This equation assumes the following procedure : the manifold is filled to pressure $P_{\mathrm{N}_{2}}$ with the valve to the calibrated volume (BV12) closed, this gas is then released into the cell assembly by opening BV13. BV13 is then closed. When the cell is cooled to $4 \mathrm{~K}$, as will be done in the next step, the nitrogen in the cell assembly will all condense into the bottom of the target chamber.

The next step is to cool the cell to $4 \mathrm{~K}$ using liquid helium. This is done because the cell pressure must be below $1 \mathrm{~atm}$ while removing the cell from the cell assembly. Removing the cell, known as the pull-off, requires the use of a methane/oxygen torch to melt the glass tubing between the cell and the cell assembly. Keeping the cell pressure below $1 \mathrm{~atm}$ ensures that the helium pressure will collapse the molten glass in a self-sealing manner, keeping out atmospheric gases. Failure to do this, will cause the high-pressure helium to punch a hole at the point of the pull-off and the ${ }^{3} \mathrm{He}$ gas will leak out into the atmosphere.

A stainless steel dewar filled with liquid helium is used to cool the cell as shown in shown in Fig. 6.9. The dewar, custom-made for this system, is basically a cylindrical bucket that is designed to hold liquid helium. The walls of the dewar are 


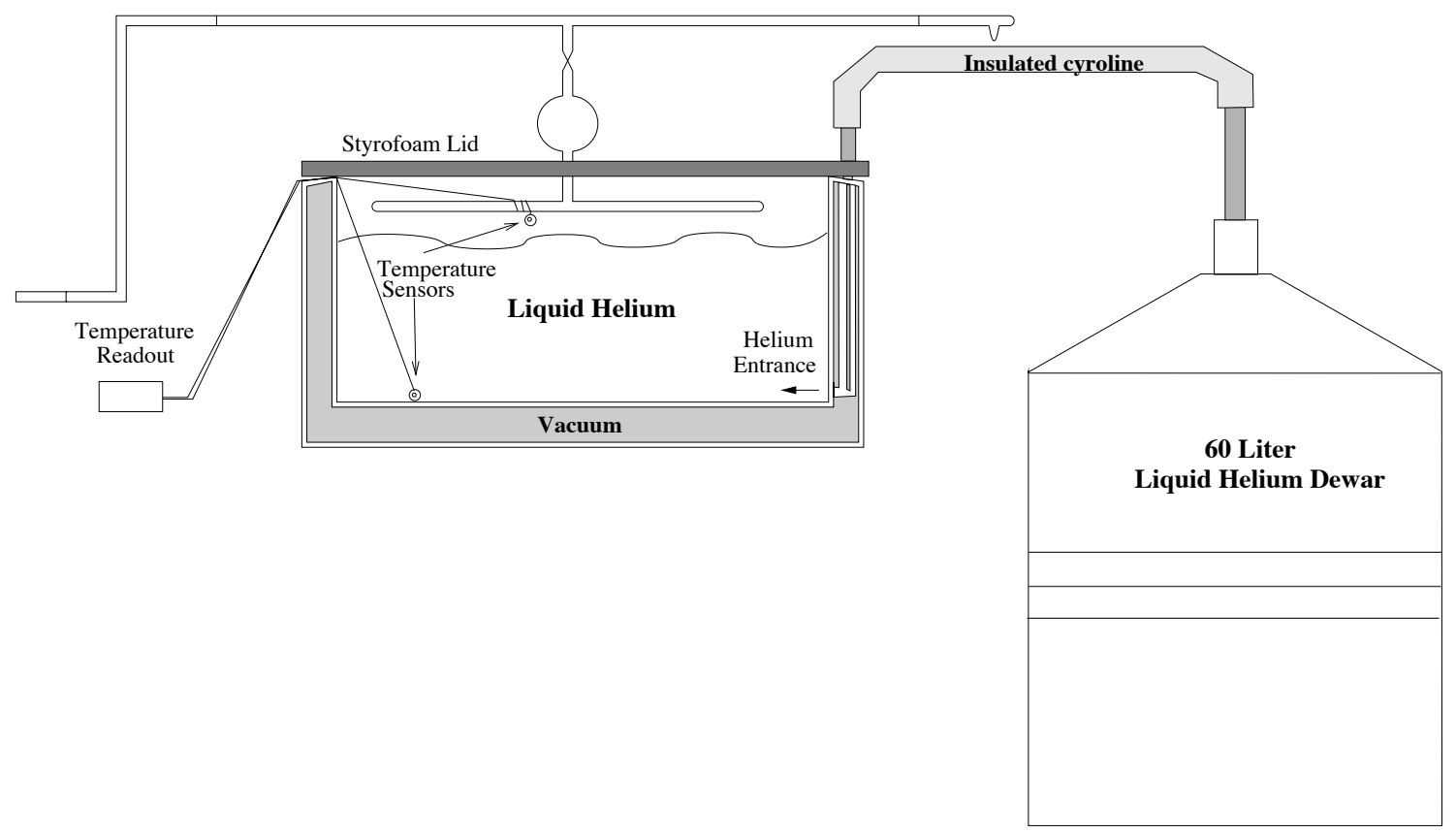

Figure 6.9: Cyrocooling Configuration

superinsulated and are evacuated to minimize thermal contact with the environment. The lid of the dewar is made of a non-descript piece of Styrofoam which helps insulate the helium liquid, though it is not air-tight and leaks quite a bit of cold gas.

The dewar has a tube inside of it which allows an insulated cyroline to be inserted and the helium from the cyroline to enter the bottom of the dewar. This cryoline is connected to a $60 \mathrm{~L}$ storage dewar of liquid helium. The flow of liquid helium to the target dewar is increased by back pressure from a gaseous helium line into the storage dewar.

It usually takes from 10-20 minutes to fill the dewar with liquid depending on the flow of helium. The temperature is measured by a pair of cryogenic temperature sensors, one placed on the cell and one placed at the bottom of the dewar. Once the helium reaches $4 \mathrm{~K}$, the temperature is rather stable and the dewar can maintain that temperature for an hour or more.

The ${ }^{3} \mathrm{He}$ gas is then put into the cell assembly in a manner similar to the nitrogen. The gas is filtered through a heated getter into the manifold. Because 
higher pressures are necessary, the calibrated volume is opened. The goal of E97103 was to create cells with a number density of 8.5 Amagats (An Amagat is the unit-less ratio of the number density of a gas over the number density of an ideal gas at $0^{\circ} \mathrm{C}$ and $1 \mathrm{~atm}$. A gas at 1 Amagat has a number density of $2.689 \times 10^{19}$ atoms $\left./ \mathrm{cm}^{3}\right)$. The cells were filled with two charges of ${ }^{3} \mathrm{He}$ so that the pressure did not become too high for the instrumentation. The formula for calculating the final number density is:

$$
n_{3 \mathrm{He}}=\frac{273.16}{T_{\mathrm{M}} V_{\mathrm{C}}}\left[\left(P_{i}^{1}-P_{f}^{1}+P_{i}^{2}-P_{f}^{2}\right)\left(V_{M}+V_{C V}\right)-V_{S} P_{f}^{2}\right]
$$

where $n_{3} \mathrm{He}$ is the ${ }^{3} \mathrm{He}$ density in Amagats, $P_{i}^{1}$ and $P_{i}^{2}$ are the manifold pressures before opening the valve (BV13) to the cell assembly for the first and second charges respectively, $P_{f}^{1}$ and $P_{f}^{2}$ are the manifold pressures after the valve (BV13) to the cell assembly and $T_{M}$ is the temperature of the manifold.

Once the proper amount of ${ }^{3} \mathrm{He}$ is in the cell then it can be separated from then assembly with the methane/oxygen torch. The tubing at the pull-off point has been narrowed by the glass blower to make the separation easier. The person melting the glass must be sure to heat the glass evenly and to anneal the glass around the pull-off point on the cell after it has been separated. Both of these things reduce cracking that can occur when it cools. The liquid helium lines are removed from the dewars, the cell is covered and generally is left over night to warm up. Then the cell is ready to be characterized and tested.

\subsection{Characterizing Target Cells}

\subsubsection{External Dimensions of Cells used during E97-103}

There were two ${ }^{3} \mathrm{He}$ cells used during E97-103, named Shapiro and Virginia One. Also, two other cells filled with water were used to calibrate the NMR system. The external dimensions of each cell was measured with a caliper. For the most 


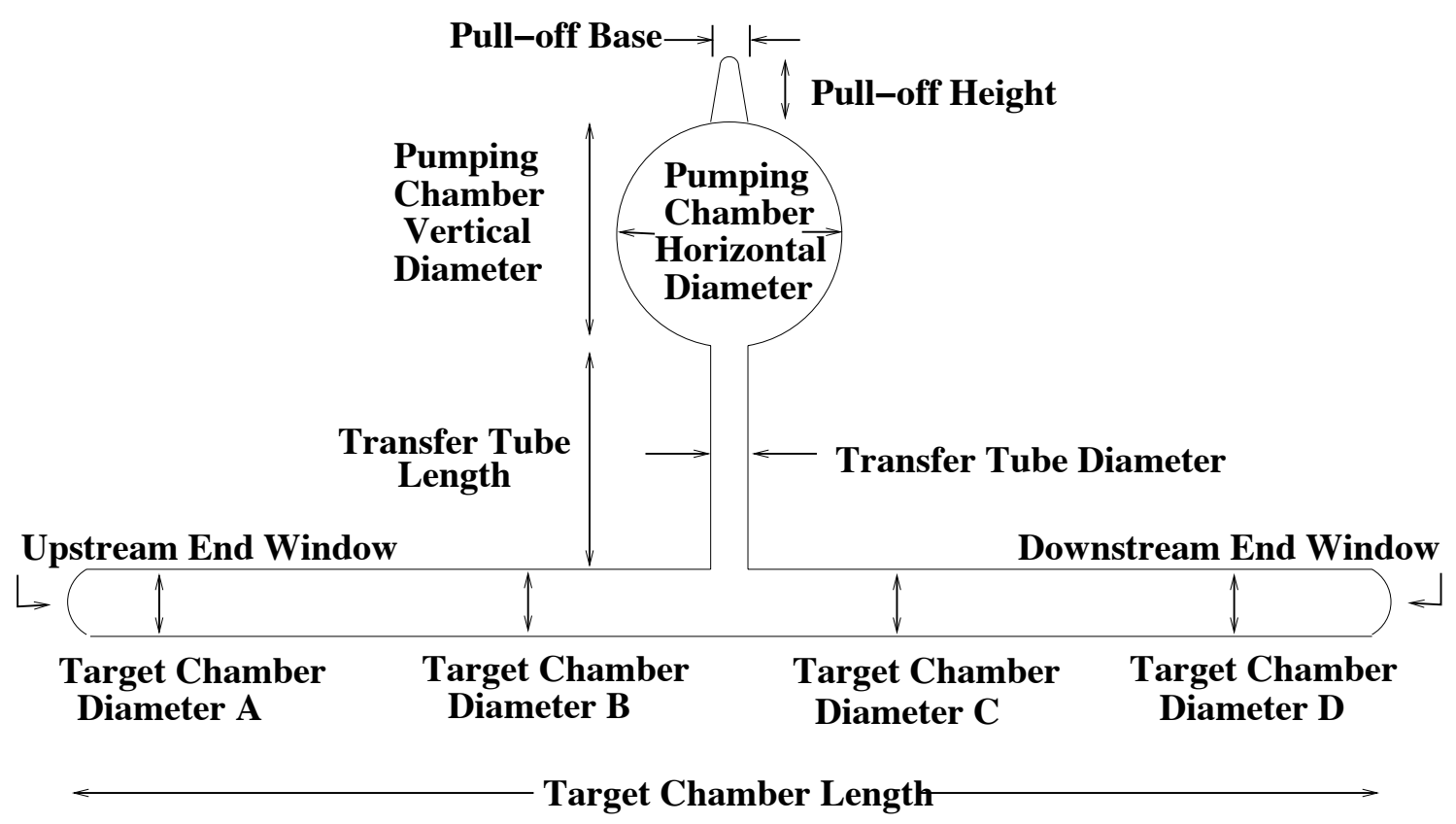

Figure 6.10: Names for the cell dimensions

\begin{tabular}{|l|cccc|c|}
\hline \hline Dimension & Shapiro & Virginia One & $25 \mathrm{~cm}_{2} \mathrm{O}$ & $40 \mathrm{~cm} \mathrm{H}_{2} \mathrm{O}$ & Uncertainty \\
\hline Pull-Off Height & 2.86 & 3.62 & 1.59 & 1.54 & 0.16 \\
P. C. V. Diam. & 6.52 & 6.74 & 6.62 & 6.72 & 0.18 \\
P. C. H. Diam. & 6.48 & 6.34 & 6.233 & 6.505 & 0.020 \\
T. Tube Diam. & 1.11 & 1.06 & 1.111 & 1.254 & 0.026 \\
T. Tube Len. & 6.46 & 6.52 & 6.380 & 6.46 & 0.020 \\
T. C. Diam. A & 1.91 & 1.85 & 1.880 & 1.871 & 0.010 \\
T. C. Diam. B & 1.88 & 1.92 & 1.865 & 1.946 & 0.010 \\
T. C. Diam. C & 1.88 & 1.91 & 1.867 & 1.927 & 0.010 \\
T. C. Diam. D & 1.93 & 1.92 & 1.878 & 1.928 & 0.010 \\
T. C. Length & 39.7 & 39.4 & 24.9 & 40.0 & 0.16 \\
\hline \hline
\end{tabular}

Table 6.1: A list of external cell dimensions and their uncertainties. All values in centimeters. 


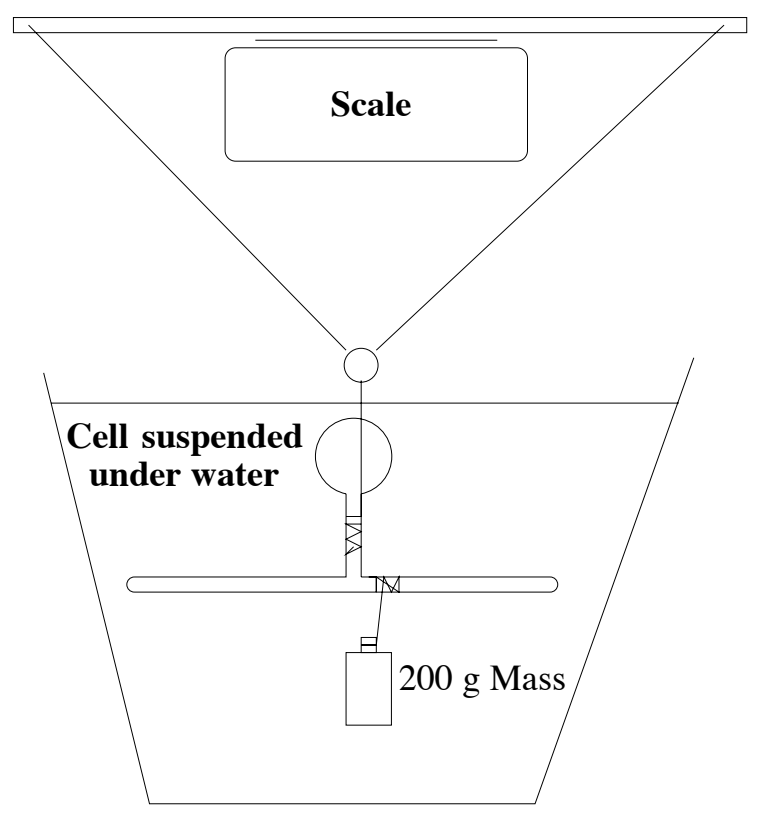

Figure 6.11: The setup for volume measurement using the Archimedes method

part, this is an accurate method since the caliper can make accurate measurements at the 10-20 micron level. However, some of the dimensions have to be estimated (for instance where the top of the pumping chamber turns into the pull-off) and measurement error contributes to the uncertainty. The other source of uncertainty is variation along the surface of the glass, for instance in the case of the target chamber diameter. Fig. 6.10 displays the position of measurements. The table 6.1 lists all the measurements and their uncertainties.

\subsubsection{Measuring the Total Internal Cell Volume}

Archimedes principle says that the buoyant force of an object in water is equal to the gravitational force of the of water it displaced. Using the density of water, one can calculate the displacement volume from the buoyant force and this volume is equal to the volume of the object. This method is used to determine the external volume of the cell.

The buoyant force in this case is the difference in weight of the cell sitting on a 
scale and the weight of the cell submerged in water. Since the cell floats in water, one must attach a weight to sink the cell. The buoyancy of this weight and the wire used to support the cell and weight, must be measured separately. The buoyant force can be calculated using the following formula:

$$
F_{B}=g\left(m_{\text {cell }}+m_{\text {app }}-m_{\text {sub }}\right)
$$

where $F_{B}$ is the buoyant force, $m_{\text {cell }}$ is the mass of the cell, $m_{\text {app }}$ is the mass of the submerged weight and wire not including the cell, $m_{\text {sub }}$ is the mass of the submerged cell plus apparatus and $g$ is gravitational acceleration [53].

Since the density of water and the density of the aluminosilicate glass are well known, to get the internal cell volume one uses the formula :

$$
V_{\text {in }}=\frac{F_{B}}{\rho_{\text {water }} g}-\frac{m_{\text {cell }}-m_{\mathrm{Rb}}-m_{3_{\mathrm{He}}}}{\rho_{\mathrm{GE} 180}}-\frac{m_{\mathrm{Rb}}}{\rho_{\mathrm{Rb}}}
$$

where $V_{\text {in }}$ is the internal volume of the cell, $F_{B}$ is the buoyant force, $\rho_{\text {water }}$ is the density of water $\left(0.9984 \mathrm{~g} / \mathrm{cm}^{3}\right), m_{\mathrm{Rb}}$ is the estimated mass of the rubidium in the cell(typically $0.3 \mathrm{~g}$ ), $m_{3 \mathrm{He}}$ is the mass of the helium gas inside the cell (typically $0.2 \mathrm{~g}), \rho_{\mathrm{GE} 180}$ is the density of the glass $\left(2.76 \mathrm{~g} / \mathrm{cm}^{4}\right.$, acquired from a combination of GE literature and Archimedes measurements on just the glass) and $\rho_{\mathrm{Rb}}$ is the density of solid $\mathrm{Rb}[53]$.

Two sets of measurements were done : one at the University of Virginia and on at the College of William and Mary. Results are given in Table 6.2, and show a systematic difference of $\approx 0.5 \mathrm{ml}$. The uncertainty of this measurement is approximately $0.3 \%$.

\subsubsection{The External Volumes of the Cell Chambers}

The external volumes of the different chambers can be used to determine their corresponding internal volumes. The internal volumes need to be known so that the 


\begin{tabular}{|l|c|c|}
\hline Cell Name & UVa Volume $\left(\mathrm{cm}^{3}\right)$ & W \& M Volume $\left(\mathrm{cm}^{3}\right)$ \\
\hline Shapiro & 201.6 & 201.1 \\
Virginia One & 195.0 & 194.4 \\
\hline
\end{tabular}

Table 6.2: The Archimedes volume measurements done at the University of Virginia and the College of William and Mary.

density differences in the cell when it is being polarized can be determined. For each cell there are three chambers of interest: the target chamber, the pumping chamber and the transfer tube. The volume of the pumping chamber can be estimated with the formula:

$$
V_{\text {pc ext }}=\frac{\pi d_{\mathrm{pc} \mathrm{vert}} d_{\mathrm{pc} \mathrm{horiz}}^{2}}{6}
$$

where $V_{\mathrm{pc}}$ ext is the external volume of the pumping chamber, $d_{\mathrm{pc}}$ vert is the vertical diameter of the pumping chamber, $d_{\mathrm{pc}}$ horiz is the horizontal diameter of the pumping chamber.

The external volume of the transfer tube is just a cylinder and can be easily calculated. The target chamber is mostly cylindrical, but because of the end windows are curved at the end and the diameter of the cylinder is non-uniform the following formula is used:

$$
V_{\mathrm{tc} \mathrm{ext}}=\frac{\pi d_{\mathrm{avg}}^{2}\left(L-2 l_{\mathrm{ew}}\right)}{4}+\frac{3 \pi d_{\mathrm{avg}}^{2} l_{\mathrm{ew}}}{8}
$$

where $V_{\text {tc ext }}$ is the external volume of the target chamber, $d_{\text {avg }}$ is the average diameter of the target chamber and $l_{\mathrm{ew}}$ is the approximate length of the curved end windows (usually around $0.8 \mathrm{~cm}$ ). The first term is the volume from the cylindrical part of the target chamber. The second term is an approximation to the volume of the end windows. It is $3 / 4$ of the volume of the cylinder of the length and diameter of the end windows. This is a crude approximation, but the error is less than a half a milliliter so a more refined model is unnecessary.

The volume of the pull-off varies from half a milliliter to one milliliter. It is ignored for this purpose. The external volumes of each cell is given Table 6.3. The 


\begin{tabular}{|l|cccc|c|}
\hline \hline Chamber & Shapiro & Virginia One & 25cm Water & 40cm Water & Uncertainty \\
\hline Pumping & 143.3 & 141.9 & 134.7 & 148.9 & 4.0 \\
Transfer & 6.25 & 5.75 & 6.18 & 7.98 & 0.35 \\
Target & 111.4 & 110.6 & 67.1 & 114.0 & 1.4 \\
\hline Total Vol. & 261.0 & 258.3 & 208.8 & 271.4 & 4.3 \\
\hline Meas. Vol. & 262.5 & 257.5 & - & - & 1.0 \\
\hline
\end{tabular}

Table 6.3: External Volumes of the cells' chambers. All values in milliliters.

\begin{tabular}{|l|ccc|c|c|}
\hline \hline Chamber Name & Nitric Acid & Water & Methanol & Average & Uncertainty \\
\hline Target Chamber & 50.07 & 49.87 & 49.87 & 49.94 & 0.10 \\
Transfer Tube & 3.34 & 3.33 & 3.68 & 3.45 & 0.10 \\
Pumping Chamber & 106.57 & 106.75 & 107.30 & 106.87 & 0.3 \\
\hline \hline
\end{tabular}

Table 6.4: Volume of the three chambers of the $25 \mathrm{~cm}$ water cell. All values are in milliliters.

calculated values are compared to the measured external volume acquired from the Archimedes method.

\subsubsection{The dimensions of the $25 \mathrm{~cm}$ Water Cell}

The $25 \mathrm{~cm}$ water cell is unique because it had the volumes of its chambers measured carefully before being sealed. This is useful in determining which methods of estimating the internal volumes of the other cells is the most accurate.

The volumes were determined by first weighing the empty cell. Then enough liquid would be added to fill the target chamber. The cell would then be weighed again. While keeping the previous amount of liquid in the target chamber, the transfer tube would be filled with additional liquid and weighed. Finally, the cell would be filled completely and weighed again. Three liquids were used all with well known densities. The average result of these liquids are used as the volume. The results are shown in Table 6.4.

\subsubsection{Target Chamber Wall Thicknesses}

The wall thickness of the target chambers used in the experiments was measured using an interferometric method using a tunable laser and a photo-detector. The 


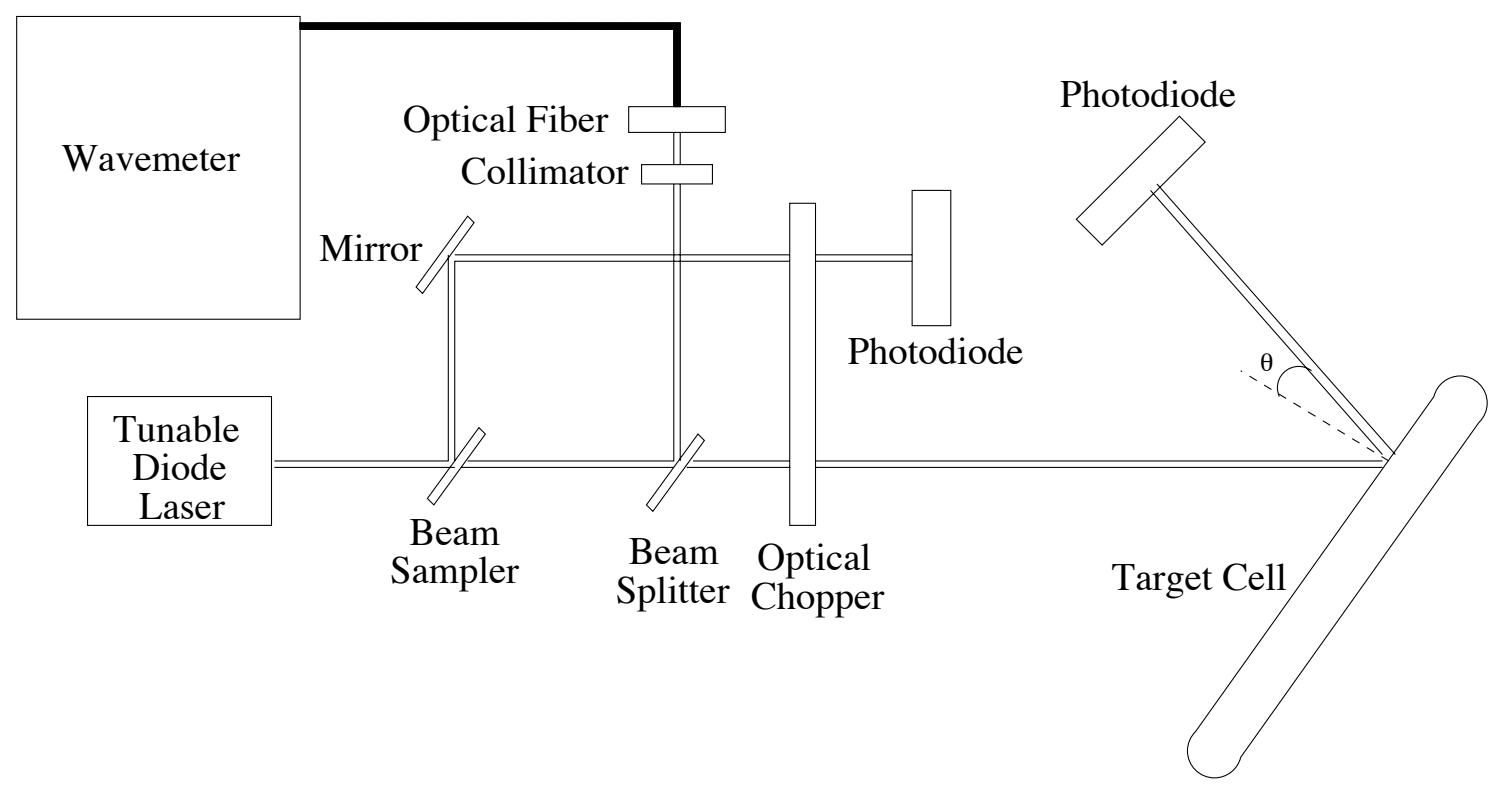

Figure 6.12: Set up for wall thickness measurements at Jefferson Lab

intensity of reflected light off of a parallel plate is given by [53]:

$$
I_{r}=\frac{\eta}{1+\eta}
$$

where

$$
\eta=\frac{4\left(\frac{n-1}{n+1}\right)^{2}}{1-\left(\frac{n-1}{n+1}\right)^{2}} \sin ^{2}\left(\frac{2 \pi n t \cos \theta}{\lambda}\right),
$$

$n$ is the index of refraction, $\lambda$ is the wavelength of the incident laser light, $\theta$ is the angle of the refracted light and $t$ is the thickness of the plate.

To measure the wall thickness with a tunable diode laser, the laser is separated into three beam paths as shown in Fig. 6.12. The first beam path is split by a beam sampler (which is a beam splitter that removes only $10 \%$ of the beam), through an optical chopper and into a photodiode. This beam is used to monitor the power of the laser. The optical chopper makes the signal from the photodiode oscillate at $1 \mathrm{kHz}$ so that a lock-in amplifier can cleanly read the signal. The second laser path goes to an optical fiber which leads to a Burleigh wavemeter. The wavemeter measures the laser wavelength to $\pm 0.0015 \mathrm{~nm}$. It is is always difficult to get enough light to the wavemeter with a low power laser so a collimator has been added to 


\begin{tabular}{|l|cccc|c|}
\hline \hline Section & Shapiro & Virginia One & $25 \mathrm{~cm} \mathrm{H}_{2} \mathrm{O}$ & $40 \mathrm{~cm}_{2} \mathrm{O}$ & Uncertainty \\
\hline A Left & 0.141 & 0.138 & 0.152 & 0.119 & 0.003 \\
A Right & 0.142 & 0.163 & 0.139 & 0.111 & 0.003 \\
B Left & 0.145 & 0.142 & 0.156 & 0.115 & 0.003 \\
B Right & 0.140 & 0.142 & 0.138 & 0.114 & 0.003 \\
C Left & 0.145 & 0.141 & 0.131 & 0.119 & 0.003 \\
C Right & 0.143 & 0.141 & 0.132 & 0.113 & 0.003 \\
D Left & 0.132 & 0.138 & 0.132 & 0.121 & 0.003 \\
D Right & 0.140 & 0.142 & 0.154 & 0.110 & 0.003 \\
\hline \hline
\end{tabular}

Table 6.5: Wall thicknesses by section. A is the upstream end, $D$ is the downstream. Left is on the side of the left spectrometer. Right is the on the side of the right spectrometer. All measurements are in centimeters.

increase the quantity of light into the wavemeter. The third path goes through an optical chopper, reflects off the target and into a photodiode. The intensity of this light will vary as the laser changes wavelengths.

The side walls of the target chamber were measured optically both at Jefferson Lab and the University of Virginia. Four to six measurements were done on each side of the cell. Because the window sections of the cells are made of a different tube than the middle part it is useful to describe the target chamber in four sections. Two represent the two window pieces on the end and two represent the middle sections separated by the transfer tube. It is found the wall thickness is uniform within an uncertainty of $30 \mu \mathrm{m}$ within each section, but sections can differ by $300 \mu \mathrm{m}$.

The measurements were not necessarily done at the same places on the cell for both Jefferson Lab and UVa measurements nor were the measurements done symmetrically (the same point on both sides). Nevertheless, the sets of measurements agreed well with a final uncertainty of 30 microns in the average wall thickness in each section of the cells. The results are given in Table 6.5.

\subsubsection{Target Chamber End Window Measurement}

The target chamber end window measurements were done at the University of Virginia using a method described in the doctoral dissertation of Ioannis Komi- 


\begin{tabular}{|l|c|c|c|c|}
\hline \hline Cell Name & $\begin{array}{c}\text { Upstream } \\
\text { Window Mark }\end{array}$ & Thickness & $\begin{array}{c}\text { Downstream } \\
\text { Window Mark }\end{array}$ & Thickness \\
\hline Shapiro & 19 & 127 & 7 & 117 \\
Virginia One & 4 & 154 & 9 & 124 \\
\hline \hline
\end{tabular}

Table 6.6: The window thicknesses in microns and their window markings.

nis [61]. This method is similar to the measurement of the wall thickness. The thicknesses are listed in table 6.6 for convenience and to document the window orientation during the experiment.

\subsubsection{Estimating the Interior Volumes of Target Cells}

The interior volumes of the targets must be accurately estimated for two reasons : to accurately estimate the target density when the cell is being polarized and to calculate the electromagnetic flux for the NMR polarimetry measurement. The method used for E97-103 uses a simple geometrical calculation for the target chamber based on the wall thicknesses and external measurements:

$$
V_{\text {Int Vol Section }}=\frac{\pi\left(d_{\text {ext }}-t_{\text {right }}-t_{\text {left }}\right)^{2} L}{16} .
$$

where $V_{\text {Int Vol Section }}$ is the internal volume for one section, $D_{\text {ext }}$ is the external diameter of that section of the target chamber, $t_{\text {right }}$ and $t_{\text {left }}$ are the right and left wall thicknesses of the section and $L$ is the length of the target chamber. This calculation was in agreement $0.5 \mathrm{ml}$ of the $25 \mathrm{~cm}$ water cell chamber. The uncertainty is set at $1.0 \mathrm{ml}$ due to lack of statistics.

The volume of the transfer tube is estimated by using external measurements, the ratio of the external to internal volume of the $25 \mathrm{~cm}$ water cell and then calculating:

$$
V_{\mathrm{tt} \text { int }}=\frac{V_{\mathrm{tt} \text { water cell int }}}{V_{\mathrm{tt} \text { water cell ext }}} V_{\mathrm{tt} \text { ext }}
$$

where $V_{\mathrm{tt}}$ int is interior volume of the transfer tube, $V_{\mathrm{tt}}$ water cell int is interior volume of the transfer tube of the $25 \mathrm{~cm}$ water cell, $V_{\text {tt }}$ water cell ext is the external volume 


\begin{tabular}{|l|cccc|c|}
\hline \hline Chamber & Gore & Tilghman & Shapiro & Virginia One & Uncertainty \\
\hline Pumping Chamber & 116.7 & 116.1 & 116.5 & 110.8 & 1.5 \\
Transfer Tube & 3.8 & 3.9 & 3.5 & 3.21 & 0.25 \\
Target Chamber & 51.1 & 53.5 & 81.2 & 80.6 & 1.0 \\
\hline Total Volume & 171.6 & 173.5 & 201.2 & 194.6 & 1.8 \\
\hline \hline
\end{tabular}

Table 6.7: The estimated chamber volumes in milliliters

Shapiro

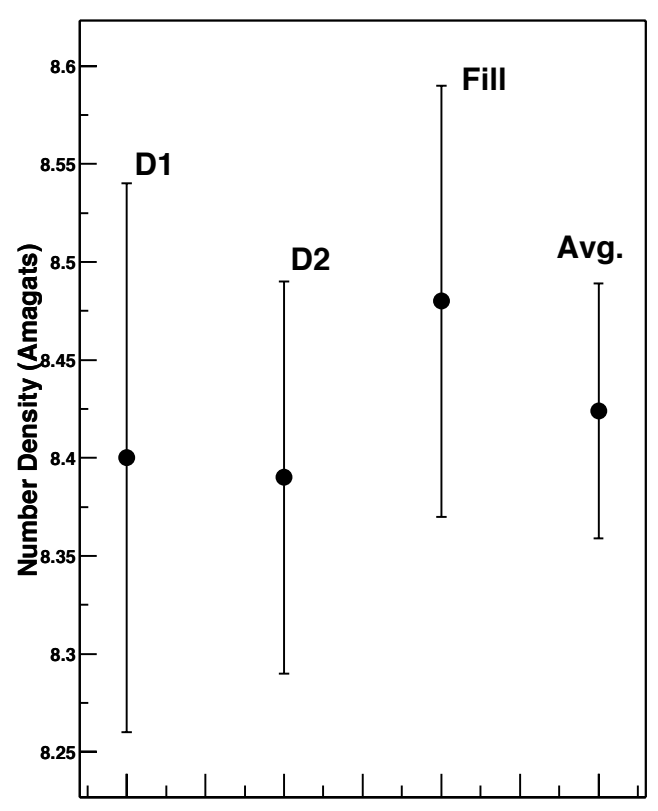

\section{Virginia One}

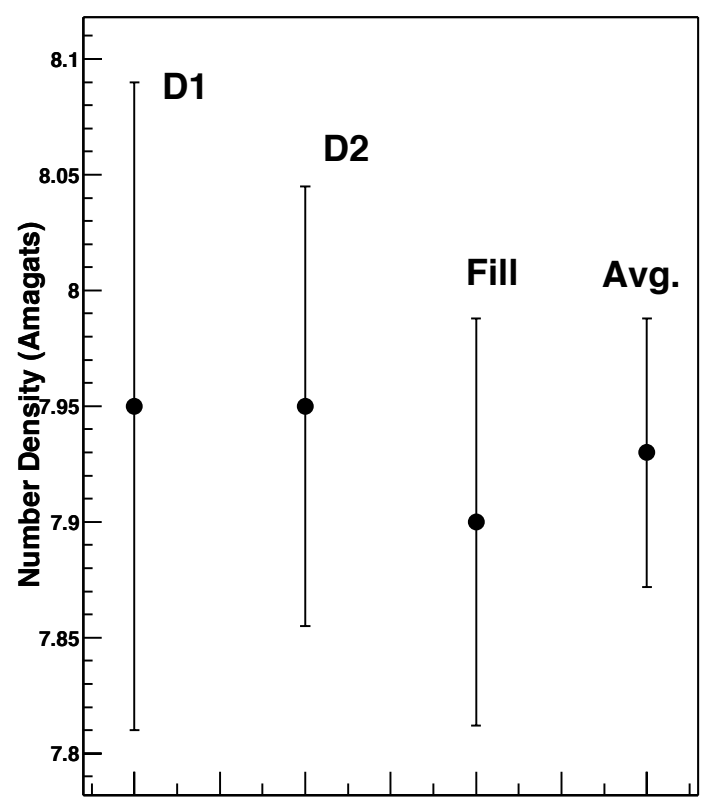

Figure 6.13: The density of the two cells used in E97-103.

of the $25 \mathrm{~cm}$ water cell and $V_{\mathrm{tt} \text { ext }}$ is the external volume of the transfer tube whose interior volume is being estimated.

The pumping chamber is estimated by subtracting these two volumes from the total internal volume estimated by the Archimedes method. The internal volumes of the chambers are presented in Table 6.7 .

\subsubsection{Measuring the Cell Density}

There are two methods used in E97-103 for measuring the ${ }^{3}$ He number density in the cells. The first is to use the density given by Eq. 6.19 with improved volume 
numbers from the Archimedes method. This second is to measure the pressure broadening of the wavelength of light absorbed by rubidium in the cell. The results of both of these methods are shown in Fig. 6.13. D1 and D2 represents the results from pressure broadening with D1 laser light and with D2 laser light. Details of the density measurement using pressure broadening is found in Ioannis Kominis' thesis [61].

\section{7 $\quad$ Lasers and Optics}

\subsubsection{Polarizing Optics}

Optical polarization of rubidium requires circularly polarized laser light. In E97-103 the laser light is provided by three Coherent diode laser systems. A diode laser produces monochromatic photons by exciting an electron transition between a p-n semi-conductor junction [65]. The light from each diode is then channeled into a fiber optic line. Since each diode provides a limited amount of power, the output fibers of many diodes are bundled into a fiber-array package (FAP). Each FAP system provides thirty watts of power and the frequency spectrum is centered at $795 \mathrm{~nm}$, with a full-width half-max of $2 \mathrm{~nm}$ at operating temperature through a single $800 \mu \mathrm{m}$ diameter output optical fiber.

The technique in E97-103 for generating circularly polarized light uses a series of optics as shown in Fig. 6.14. The light emitted by the fiber-optic is divergent, but can be made parallel by a semi-convex lens. This lens determines the size of the laser spot, typically a 3-4 cm diameter circle.

The light then enters a beam-splitter which reflects the S-wave light (light polarized perpendicular to the bottom of the beam splitter) to the right while the P-wave light (light polarized parallel to the bottom of the beam splitter) is allowed to pass through and bounce off a mirror towards the target. The reflected S-wave light

is converted to P-wave light by traveling through a quarter-wave plate, bouncing off 


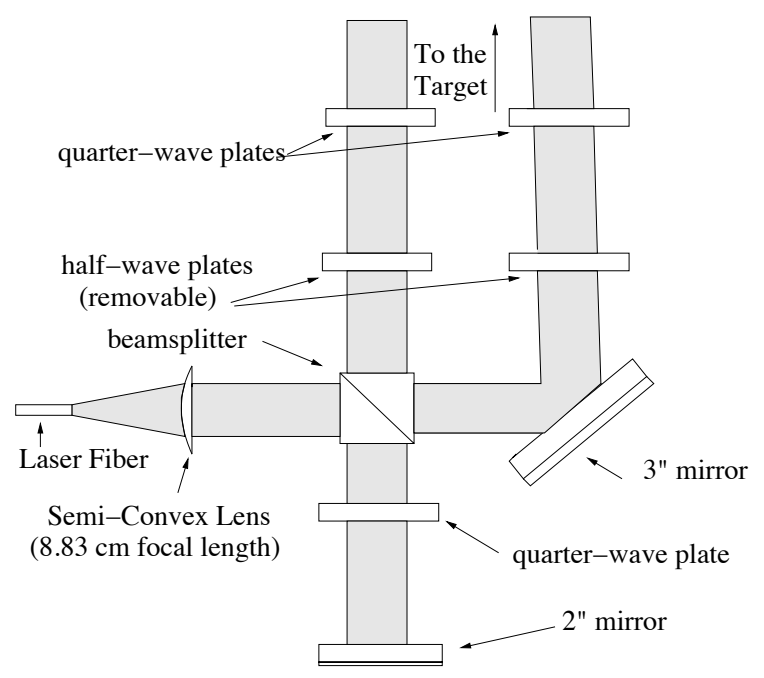

Figure 6.14: Polarizing optics for Jefferson Lab Polarized ${ }^{3}$ He target.

a mirror and returning through the same quarter-wave plate. The first trip through the quarter-wave plate converts the S-wave to circularly polarized light. The second trip converts it to $\mathrm{P}$-wave light. This $\mathrm{P}$-wave light can travel straight through the beam-splitter. However, this process is not $100 \%$ efficient and up to $10 \%$ remains S-wave and is reflected, unfortunately, back towards the laser fiber! Consequently, one has to be careful when aligning these optics since back reflection can not only damage the fiber, but also travel down the fiber and damage the diode. (A solution is to tilt the beam splitter a little.)

Both sets of P-wave light are then rotated to circularly polarized light by two quarter wave plates. This light can be converted to an opposite circular polarization by inserting half-wave plates either before the quarter-wave plates or after. The laser light is then ready to polarize rubidium.

\subsubsection{Optics Configuration}

Increasing the efficiency of these optics is important for maximum rubidium polarization with minimal laser power. The dominant cause of inefficiency is caused by the spatial profile of the light which is divergent and does not have a point-like 
source. These means all the light cannot be made perfectly parallel with just one semi-convex lens and will eventually diverge on the path to the target (which can be up to 5 meters with this target). Another important source of power loss comes from the surfaces of the optics which produce small (1\%) power losses due to reflection. These losses can be diminished, but not eliminated by coatings. The converted S-wave light will therefore generally have lower power output in a well-optimized system.

Another source of inefficiency is the orientation of the quarter- and half-wave plates. Quarter- and half-wave plates are made of crystals whose transmission velocity depends on the polarization of the normally incident light entering the crystal [65]. The axes of maximum and minimum transmission velocity are known as the fast and slow axes, respectively. To create left circularly polarized light from linearly polarized light, a quarter-wave plate needs to have its slow axis $-45^{\circ}$ from the linear polarization plane and its fast axis $45^{\circ}$ from the linear polarization plane viewing the optics towards the target. For right circularly polarized light, the fast axis is at $-45^{\circ}$ while the slow axis is at $45^{\circ}$. When using the half-wave plate, its axis must also be at a $45^{\circ}$ angle from the plane of linear polarization. Deviations from this alignment of the quarter and half-wave plates will reduce the polarization of the laser light, potentially reducing the rubidium polarization.

The optics configuration in Hall A used six beam lines: three for the lasers used for longitudinal target polarization and three lasers for transverse target polarization, as shown in Fig. 6.15. There was also a spare beam line which was not used. The polarizing optics and the optical fibers from the lasers are mounted in parallel on three-foot tall 2" diameter metal poles. The longitudinal laser paths are on top and use two mirrors at the target to align the light along the longitudinal holding field. The transverse laser paths are the bottom set of optics and travel straight into the pumping chamber through a hole in the side of the target cover. 


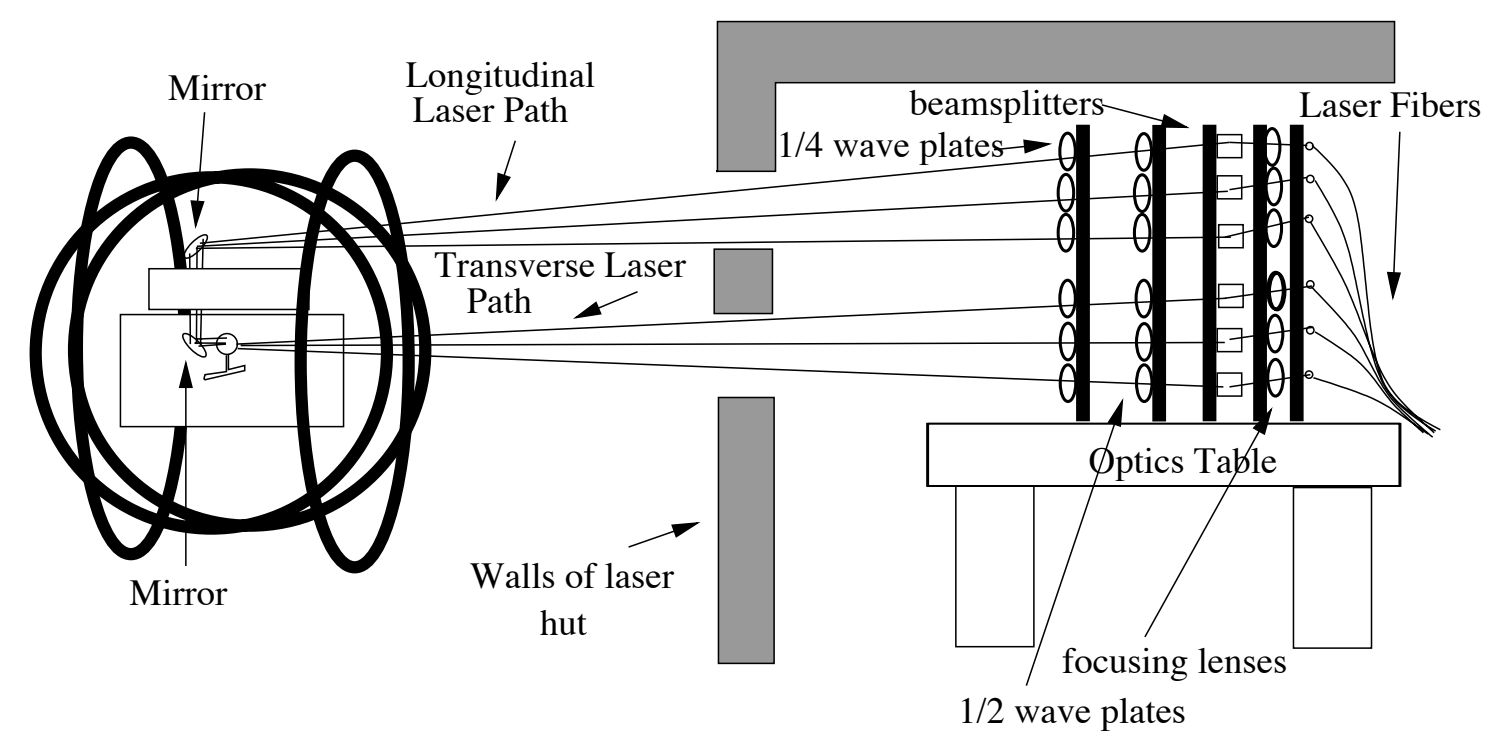

Figure 6.15: A diagram of Laser Optics Configuration in Hall A.

\subsection{The Magnetic Fields}

\subsubsection{The Helmholtz Coils}

The Helmholtz coils provide the holding field for the polarized ${ }^{3} \mathrm{He}$ target. There are two sets of coils that can be used in combination to form a uniform holding field around the target cell that can be in any direction in the scattering plane. In E97103 the coils were arranged so that the smaller coil's field was along the path of the electron beam and the larger coil's field was perpendicular to it, as shown in Fig. 6.16 .

The smaller coils have an interior radius of $63.3 \mathrm{~cm}$ and are made of 256 windings of wire in each coil. The larger coils have an interior radius of $72.4 \mathrm{~cm}$ and are made of 272 windings [66]. Each set of coils is powered by a KEPCO Model BOP 3612D power supply. The maximum current of the power supply is 10 Amps with a maximum voltage of $32 \mathrm{~V}$. The power supplies are run in voltage mode which means the power supply provides constant voltage with slightly fluctuating current. It would be better to run in current mode, but these particular power supplies create a high-frequency noise when in current mode making NMR polarimetry measurements 


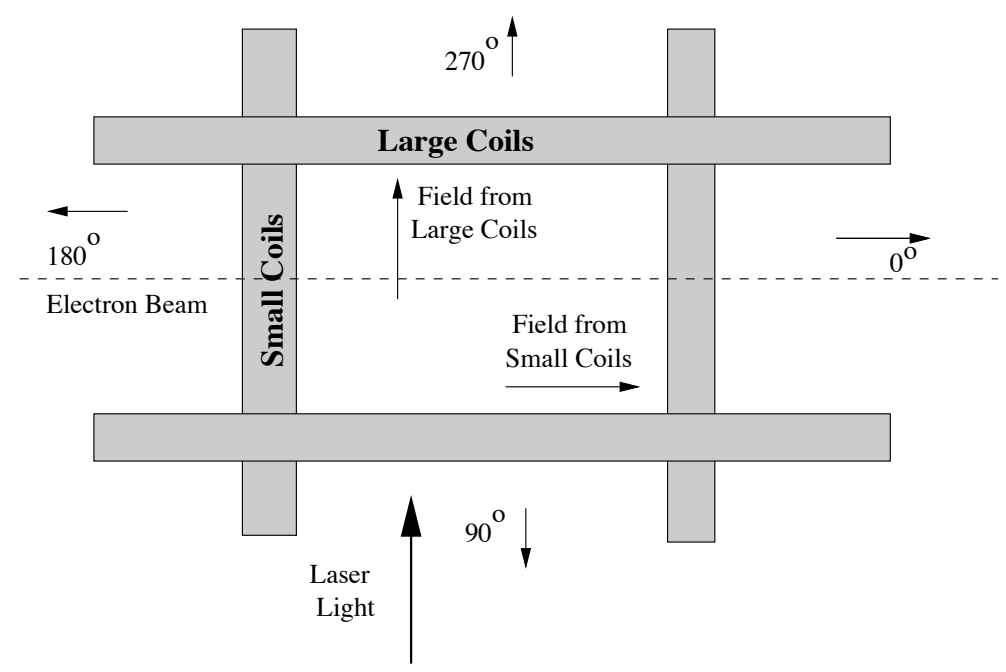

Figure 6.16: Helmholtz coils configuration with respect to beam line and lasers.

impossible [67].

The voltage output in each KEPCO power supply is controlled by a SRS DS345 function generator. These two function generators are in turn controlled through a GPIB interface to a Windows-based PC running LabView. The output voltage of the DS345 must be calibrated to get a meaningful magnetic field out of the coils. It also useful to know the current calibration, so that a relation between the magnetic field and the current is known. This is useful for monitoring the magnetic field.

One set of coils is calibrated at a time. Since the coils are warmer when current has been running through them, the coils are calibrated at high current (warm coil calibration) and at low current (cold coil calibration). The warm coil calibration is used whenever the coils are above 1 Amp. The relations between the coils and their current and magnetic field are written:

$$
\begin{aligned}
I & =\alpha_{I} V_{p p}+\beta_{V} \\
B & =\alpha_{B} V_{p p}+\beta_{V} \\
B & =\alpha_{B I} I+\beta_{B I}
\end{aligned}
$$

where I is the current in the coils, $\alpha_{I}$ and $\alpha_{B}$ are the slope constants for the calibra- 


\begin{tabular}{|l|cccccc|}
\hline \hline Calibration & $\begin{array}{c}\alpha_{I} \\
(\mathrm{Amps} / \mathrm{V})\end{array}$ & $\begin{array}{c}\beta_{I} \\
(\mathrm{Amps})\end{array}$ & $\begin{array}{c}\alpha_{B} \\
(\mathrm{G} / \mathrm{V})\end{array}$ & $\begin{array}{c}\beta_{B} \\
(\mathrm{G})\end{array}$ & $\begin{array}{c}\alpha_{B I} \\
(\mathrm{G} / \mathrm{Amps})\end{array}$ & $\begin{array}{c}\beta_{B I} \\
(\mathrm{G})\end{array}$ \\
\hline Cold Small Coils & -1.164 & 0.078 & 4.123 & -0.277 & -3.542 & -0.001 \\
Warm Small Coils & -1.156 & 0.180 & 4.026 & -0.633 & -3.483 & -0.007 \\
Cold Large Coils & -1.202 & 0.084 & 4.018 & -0.558 & -3.343 & -0.278 \\
Warm Large Coils & -1.152 & 0.124 & 3.962 & -0.718 & -3.439 & -0.292 \\
\hline \hline
\end{tabular}

Table 6.8: A list of the constants used to calibrate the Helmholtz Coils in E97-103.

tions, $\beta_{I}$ and $\beta_{B}$ are the offset constants for the calibrations, $\alpha_{B I}$ and $\beta_{B I}$ are the calibration constants between the current and voltage and $V_{p p}$ is the voltage output of the DS345 function generator. The results of these calibrations are in the Table 6.8 and plots can be found in Appendix A.

The magnetic field measurements are done with two Gaussmeters. The cold coil measurements are done with an extremely sensitive Gaussmeter, but could only measure up to 1 Gauss. The other Gaussmeter, made by Lake Shore, could measure much higher fields than was necessary, but was only precise to 0.010 Gauss. It is unclear whether the difference in calibrations in warm and cold coils is due to the differences in the actual calibration or simply differences in the Gaussmeters.

\subsubsection{Mapping of Helmholtz Coils}

There are two reasons to that make it important to control holding field gradients in a polarized ${ }^{3} \mathrm{He}$ target. The first and most important is that field gradients over 20 mGauss/cm for 25 Gauss field will increase polarization losses during polarimetry measurements that depend on adiabatic fast passage (AFP). Both of the polarimetry methods used on the Jefferson Lab polarized ${ }^{3}$ He target (NMR and EPR) depend on AFP. The second reason is that field gradients over $100 \mathrm{mGauss} / \mathrm{cm}$ for a 25 Gauss field will begin lowering the maximum polarization of the target cell. Obviously, this is a less important reason because performance is already affected at $20 \mathrm{mGauss} / \mathrm{cm}$. 


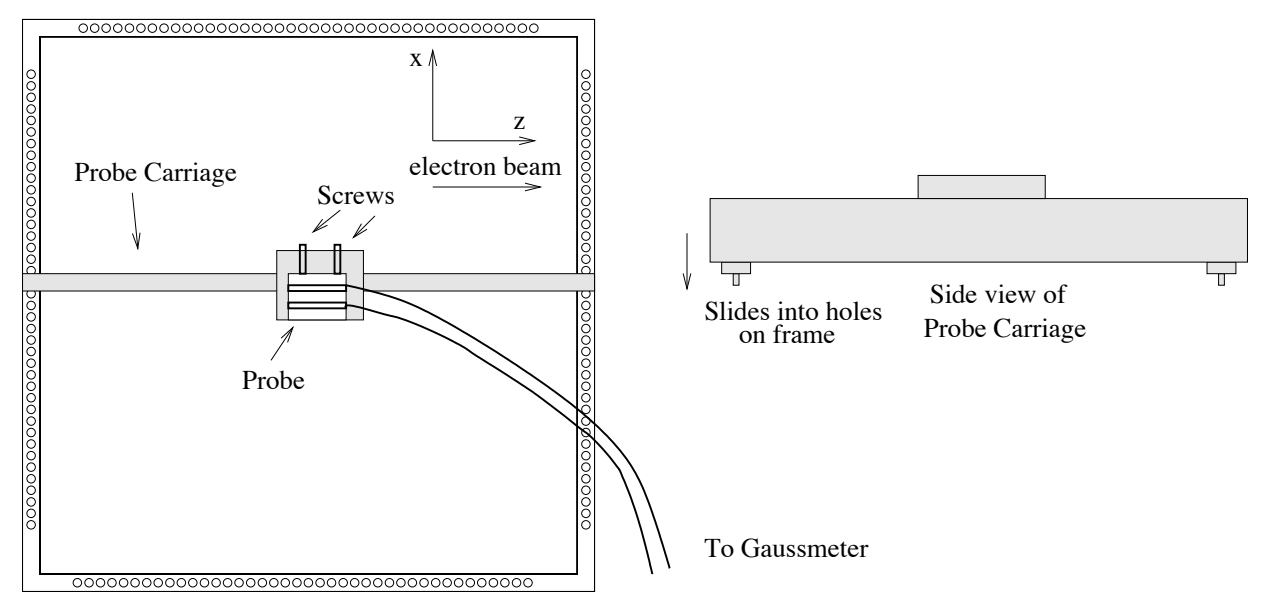

Figure 6.17: Diagram of frame and probe carriage used to map holding field.

The field gradients are measured using the two Gaussmeters used to calibrate the field. The Lakeshore Gaussmeter is used to measure fields over 1 Gauss and the sensitive Gaussmeter is used for all other measurements. The probes for the gaussmeter are fastened to the probe carriage pictured in Fig. 6.17. The entire carriage sits on an aluminum frame that sits in the target chamber. The frame is centered where the target would be when it is in beam. The carriage can be positioned accurately on the frame by the precisely machined holes. There are 40 holes on each side of the frame with a $1 \mathrm{~cm}$ separation between them.

These field measurements could only be done in the $x$ and $z$ directions, though for a full measurement one would also like to do vertical measurements. When the probe is aligned perpendicular to the holding field it is particularly sensitive to small changes in angle. For the $x$ direction only points in the center chamber matter since the cell is only $<2 \mathrm{~cm}$ wide in $x$. However, the cell extends from $-20 \mathrm{~cm}$ to $20 \mathrm{~cm}$ in $z$ (in this coordinate system); therefore all the gradients in $z$ are important.

The measured gradients for the small coil are presented in Appendix A. There seems to be a large gradient on the negative edge of $\frac{d B_{z}}{d z}$ for the small coil and $\frac{d B_{x}}{d x}$ for the large coil. Since these are along the axis of the holding field the field should be symmetric. Its unclear where the source of this gradient. Some data points on the 

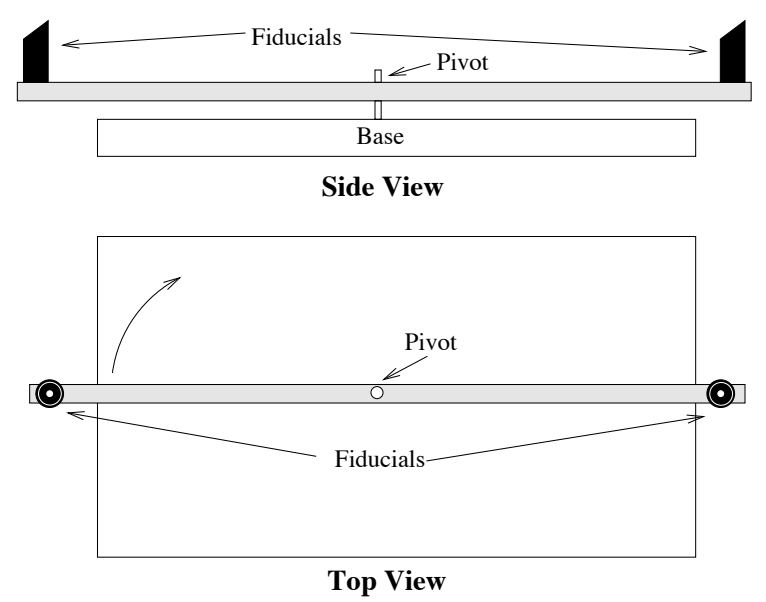

Figure 6.18: Compass used to measure the holding field direction.

edge of $x$ have been ignored since they are outside the cell area and don't indicate a larger trend.

\subsubsection{Field Direction of the Helmholtz Coils}

To measure $g_{2}^{n}$ both longitudinal and transverse asymmetries must be measured. The expected physics asymmetries showed that the longitudinal asymmetry was an order of magnitude larger than the transverse asymmetry. Therefore, if the holding field direction was not exactly aligned with the electron beam then there could be a significant amount of longitudinal asymmetry mixed in with the transverse asymmetry.

To maximize accuracy of the holding field direction, a set of measurements were done with a long compass and a survey team to measure the holding field angle. The compass, shown in Fig. 6.18, is a rectangular iron rod. A set of fiducials are placed on either end of the rod. The rod of the compass is set on a pivot about which is can swing freely. The compass is place inside the target scattering chamber. When the magnetic field is set to the desired direction, a survey team can measure the absolute position of the fiducial in reference to set locations in Hall A to 0.2 $\mathrm{mm}[68]$. 


\begin{tabular}{|c|cc|cc|}
\hline \hline $\begin{array}{c}\text { Nominal } \\
\text { Angle }\end{array}$ & $\begin{array}{c}\text { Survey 3 } \\
\text { Angle }\end{array}$ & $\begin{array}{c}\text { Calibration } \\
\text { Angle }\end{array}$ & $\begin{array}{c}\text { Survey 4 } \\
\text { Angle 1 }\end{array}$ & $\begin{array}{c}\text { Calibration } \\
\text { Angle 2 }\end{array}$ \\
\hline 0 & 0.02 & 0.218 & -0.480 & -0.262 \\
90 & 89.92 & 88.93 & 89.09 & 88.93 \\
180 & 180.17 & 179.96 & 180.15 & 179.96 \\
270 & 270.02 & 269.44 & 269.59 & 269.46 \\
\hline \hline
\end{tabular}

Table 6.9: Table of holding field angles given by two surveys and the holding field calibrations. Survey 3 was done just before E97-103 and Survey 4 was done just after. Calibration Angle and Calibration 2 refer to the angle given by the Helmholtz coil currents during Survey 3 and Survey 4 respectively. All angles are given in degrees.

A summary of the surveys is presented in Table 6.9. A listing of all the survey information is listed in Appendix C. The measurements from the third survey were used to align the holding field. However, the calibration from the Helmholtz disagreed with the survey by $0.5^{\circ}$ at the $270^{\circ}$ setting and $1.0^{\circ}$ in the $90^{\circ}$ setting. Unfortunately, the measurements from the final survey agree within $<0.3^{\circ}$ with the calibration of the Helmholtz coils, contradicting the previous measurement. It also agrees well with the first survey done in May. It is unclear what is the source of the discrepancy. The effect of this discrepancy on E97-103's results will be discussed in the asymmetry analysis chapter.

\subsubsection{Calibration of RF coils}

There are two radio frequency $(\mathrm{RF})$ coils that are used to create a highfrequency magnetic field of up to 100 mGauss. The coils are powered by a Hewlett Packard Model 355 D function generator and a ENI - 2100 L broadband power amplifier as shown in Fig. 6.19. A capacitor (inside the capacitor box) is added to the system to boost power in the coils by matching the impedance with the broadband amplifier. The current in the RF coils is monitored by a loop current monitor made by Pearson. The signal from the current monitor is read by an oscilloscope.

The magnitude of the field created by the RF coils is usually referred to as $H_{1}$. $H_{1}$ is calibrated by inserting a small loop of wire perpendicular to the RF field and 
Windows PC

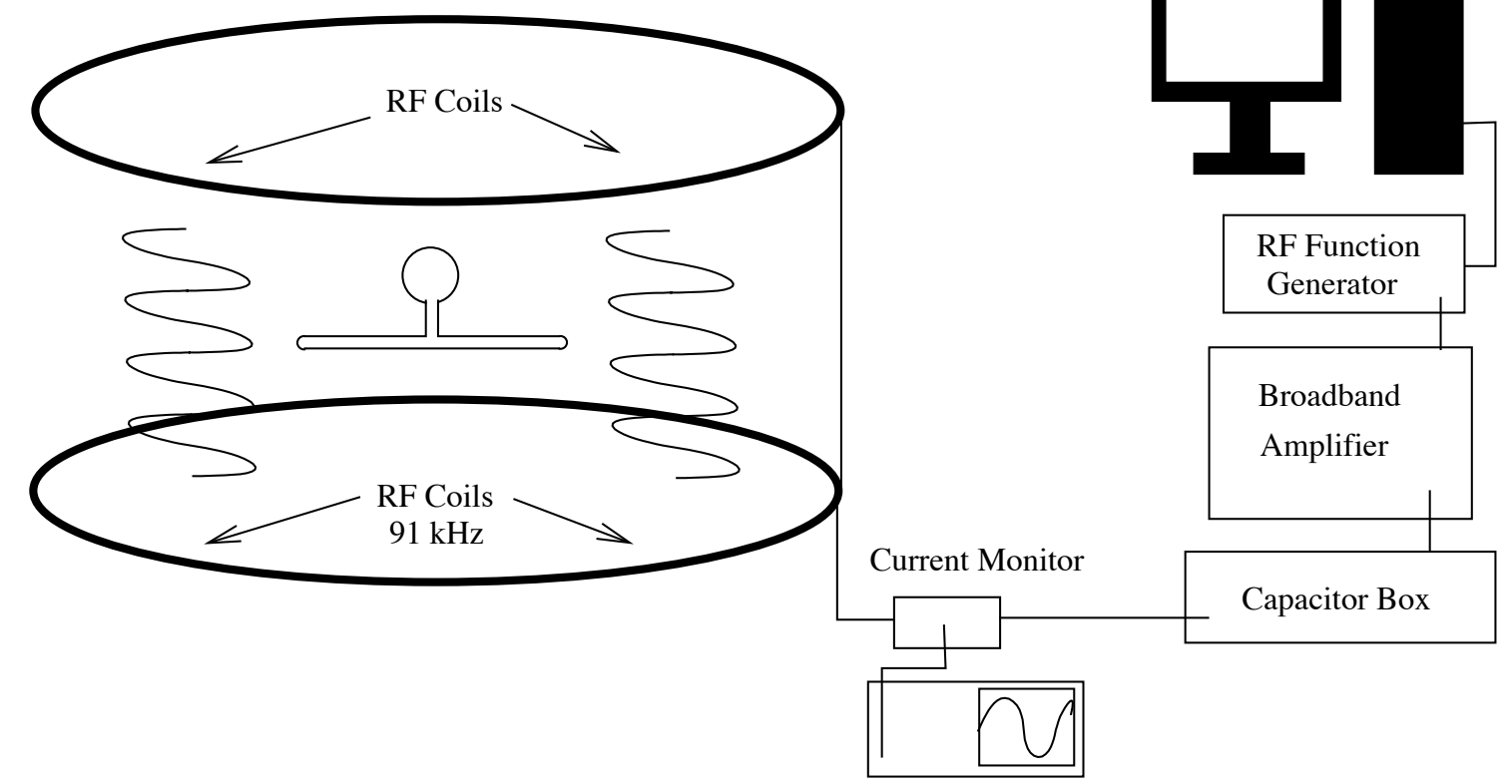

Figure 6.19: Equipment used to power and monitor RF coils.

monitoring the amplitude of the voltage that comes out of it with an. The $H_{1}$ field can be calculated using the formula:

$$
H_{1}=\frac{V_{p p}\left(10^{7} \mathrm{mGauss} / \mathrm{T}\right)}{8 \pi^{2} f N D^{2}} \frac{1}{\left|1-R_{c} / Z\right|} \approx \frac{V_{p p}\left(10^{7} \mathrm{mGauss} / \mathrm{T}\right)}{8 \pi^{2} f N D^{2}}
$$

where $H_{1}$ is the RF field amplitude, $V_{p p}$ is the peak-to-peak voltage read on the oscilloscope, $f$ is the frequency of the RF field, $N$ is the number of turns in the measurement loop, $D$ is the diameter of the measurement loop, $R_{c}$ is the resistance of the coil and $Z$ is the impedance of the coil and BNC cable used to attach it to the oscilloscope. $\left|R_{c} / Z\right|$ in the system used in E97-103 was $\ll 1.0$ and ignored.

The $H 1$ calibration is shown in Fig. 6.20. The coil of wire used for the calibration had 47 turns and a diameter of $1.25 \mathrm{~cm}$. The RF frequency used for the experiment was $91 \mathrm{kHz}$. The voltage setting on the RF FG used for AFP measurements was $2.5 \mathrm{~V}$ rms which translates from this calibration to 57.93 mGauss. 


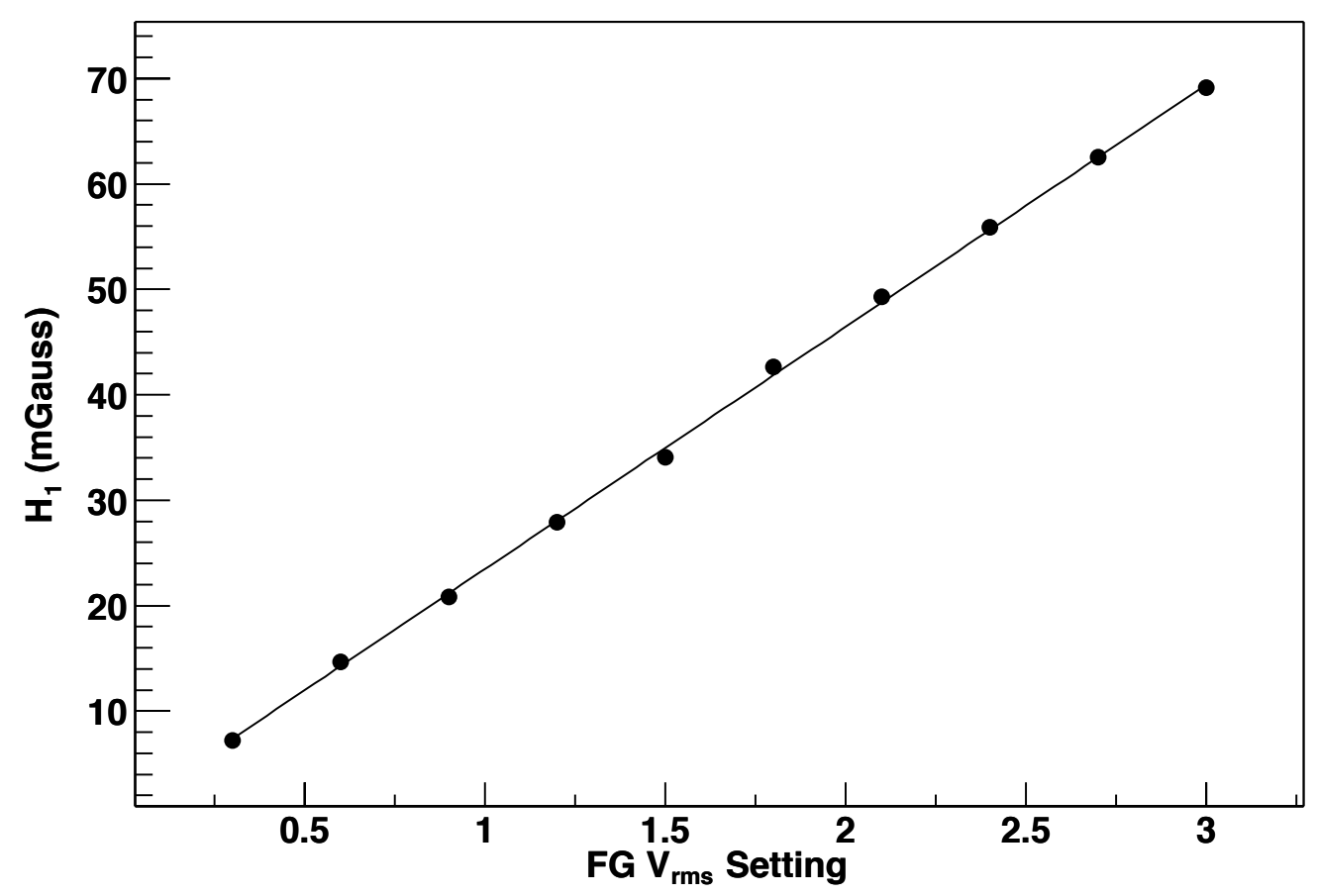

Figure 6.20: The $H_{1} R F$ field versus the voltage on the HP Function Generator.

\subsection{Measuring Target Polarization}

\subsubsection{The Adiabatic Condition}

Adiabatic Fast Passage (AFP) is a technique for reversing nuclear magnetization that is used in the two forms of polarimetry used by the polarized ${ }^{3} \mathrm{He}$ target. For AFP to work, the process of polarimetry must meet the adiabatic condition. To see how to define the adiabatic condition one must start with a free magnetization (like a ${ }^{3} \mathrm{He}$ nucleus) in a static magnetic field $\vec{H}_{0}=H_{0} \hat{k}$. The motion of the magnetization in a holding field is described classically (which is sufficient for describing the polarimetry systems) by [69]:

$$
\frac{d \vec{M}}{d t}=\gamma \vec{M} \times \vec{H}_{0}
$$

where $\gamma$ is the gyromagnetic ration $\left(3.24 \mathrm{kHz} /\right.$ Gauss for $\left.{ }^{3} \mathrm{He}[70]\right)$.

Since this motion is obviously a precession around the holding field $\vec{H}$ it is 
useful to describe the system in a rotating frame of reference $S^{\prime}$ that rotates around the holding field axis at the same frequency as the precession. Any vector, $\vec{A}$, in a rotating frame of reference can be described as [69]:

$$
\frac{d \vec{A}}{d t}=\frac{\partial \vec{A}}{\partial t}+\vec{\omega} \times \vec{A}
$$

where the first term on the right hand side is the motion in the rotating frame of reference and the second term is the motion of the frame defined by axis $\vec{\omega}$. With this relation one can define the motion of the magnetization as:

$$
\frac{\partial \vec{M}}{\partial t}=\gamma \vec{M} \times\left(\vec{H}_{0}+\frac{\vec{\omega}}{\gamma}\right) .
$$

Therefore if one picks the right frequency of rotation $\omega$ such that $\omega=-\gamma H_{0}$ the motion of the magnetization $\frac{\partial \vec{M}}{\partial t}=0$. This frequency in a static holding field is known as the Larmour frequency and is usually denoted $\omega_{0}$.

For AFP, a rotating field $\vec{H}_{1}=H_{1} \cos (\omega t) \hat{i}+H_{1} \sin (\omega t) \hat{j}$ is added to the static field $\vec{H}_{0}$, where $\omega$ is the frequency of precession of the rotating frame, which is not necessarily $w_{0}$. In the rotating frame, once can define an effecting field $\vec{H}_{e}$ which is static in this frame:

$$
\vec{H}_{e}=\left(H_{0}+\frac{\omega}{\gamma}\right) \hat{k}+H_{1} \hat{i}
$$

The magnitude of this vector is written:

$$
H_{e}=\left[\left(H_{0}+\frac{\omega}{\gamma}\right)^{2}+H_{1}^{2}\right]^{\frac{1}{2}}
$$

The next step in describing the adiabatic condition is to make the static holding field, a slowly varying field. It is useful to generalize this situation to a vector $\vec{H}$ whose time derivative can be written [69] :

$$
\frac{d \vec{H}}{d t}=\vec{\Omega} \times \vec{H}+\Omega_{1} \vec{H}
$$


where the vector $\vec{\Omega}$ is an instantaneous axis constantly along the direction of $H$ and $\Omega_{1}$ is a scaler. A collection of spins with net magnetization $\vec{M}$ in a magnetic field $H$ described above will change in time as:

$$
\frac{\partial \vec{M}}{\partial t}=\gamma \vec{M} \times\left(\vec{H}+\frac{\vec{\Omega}}{\gamma}\right) .
$$

Looking at this equation carefully, the adiabatic condition becomes apparent. If the magnitude of $\vec{\Omega}$ is much smaller than the magnitude of $\gamma \vec{H}$ then the second term in Eq. 6.38 can be ignored and the components of magnetization can be written:

$$
\begin{aligned}
& \frac{\partial M_{z}}{\partial t}=M_{x} \Omega_{y}-M_{y} \Omega_{x} \\
& \frac{\partial M_{x}}{\partial t}=\gamma H M_{y} \\
& \frac{\partial M_{y}}{\partial t}=-\gamma H M_{x}
\end{aligned}
$$

which depends on $H_{x}=H_{y}=0$ which is true by definition of the frame.

Over a long period of time the change in $M_{z}$ will be:

$$
\Delta M_{z}=M_{z}(t)-M_{z}(0)=\int_{0}^{t}\left[M_{x}\left(t^{\prime}\right) \Omega_{y}\left(t^{\prime}\right)-M_{y}\left(t^{\prime}\right) \Omega_{x}\left(t^{\prime}\right)\right] d t
$$

Since $M_{x}, M_{y}, \Omega_{x}$ and $\Omega_{y}$ are all oscillatory and if $O m e g a_{x}$ and $O m e g a_{y}$ are small then $M_{z}$ is constant with time. This means that the angle of the magnetization with the instantaneous direction of the field is a constant of the motion if the adiabatic condition, $\Omega \ll|\gamma H|$, is satisfied [69].

To derive the adiabatic condition more specifically for the purposes of the polarized ${ }^{3} \mathrm{He}$ target, one needs to go back to the effective field presented in Eq. 6.35. Assuming the holding field $\vec{H}_{0}$ changes linearly in time as $\dot{H}_{0} t$, the change in the effective filed $\vec{H}_{e}$ can be written [69]:

$$
\frac{d \vec{H}_{e}}{d t}=\cos \theta \frac{\dot{H}_{0}}{H_{e}} \vec{H}_{e}+\sin \theta \frac{\dot{H}}{H_{e}}\left(n \times \vec{H}_{e}\right)
$$

were $\theta$ is the angle between the effective field $\vec{H}_{e}$ and the holding field $\vec{H}_{0}$. The vector has been written in the frame of the normalized unit vectors $\vec{H}_{e} / H_{e}$ and $\hat{n} \times \vec{H}_{e} / H_{e}$, 


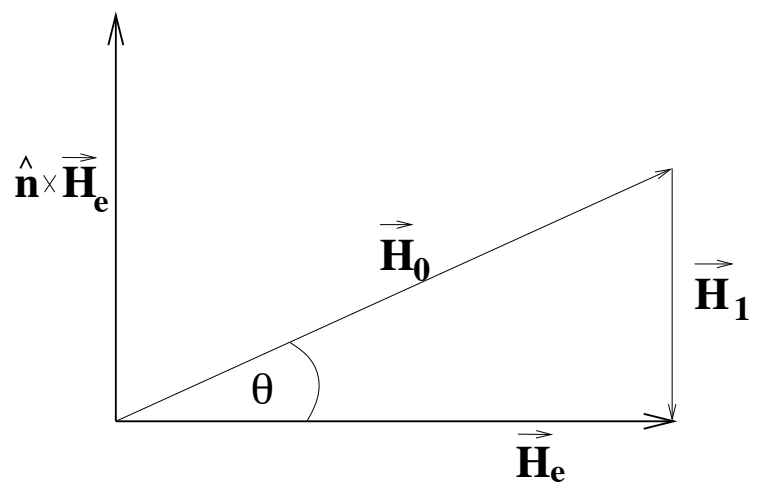

Figure 6.21: The holding field $\vec{H}_{0}$ and rotating field $\vec{H}_{1}$ in the frame of reference defined by $\vec{H}_{e}$ and $\hat{n} \times \vec{H}_{e}$.

as shown in Fig. 6.21, where $\hat{n}$ is a unit vector in the direction perpendicular to $H_{0}$ and $H_{1}$. Comparing this equation and Eq. 6.37 one can see [69]:

$$
\Omega=\sin \theta \frac{\dot{H}_{0}}{H_{e}}=H_{1} \frac{\dot{H}_{0}}{H_{e}^{2}}
$$

where $\sin \theta=H_{1} / H_{e}$ comes from the geometry in Fig. 6.21. So for the system of a changing holding $H_{0}$ and a rotating field $H_{1}$ the adiabatic condition is:

$$
\dot{H}_{0} \ll \frac{\gamma H_{e}^{3}}{H_{1}} .
$$

Near the Larmour frequency where $H_{1} \approx H_{e}$ :

$$
\dot{H}_{0} \ll \gamma H_{1}^{2} .
$$

This is the adiabatic condition for the polarized ${ }^{3} \mathrm{He}$ target. When this condition is met, the magnetization of the ${ }^{3} \mathrm{He}$ nucleus will follow the effective field. This is what allows a magnetization reversal during AFP.

\subsubsection{The Bloch Equations}

The derivation of the adiabatic condition assumed a free target in a homogeneous field. In reality, there are effects that must be added to the equation of motion presented by Eq. 6.32. A magnetization in a static field will tend towards its 
equilibrium value $M_{z}=M_{0}=\chi_{0} H_{0}$, where $\chi_{0}$ is the magnetic susceptibility, which can be described by the equation [69]:

$$
\frac{d M_{z}}{d t}=-\frac{M_{z}-M_{0}}{T_{1}}
$$

where $T_{1}$ is the the longitudinal relaxation time. Similarly, if the magnetization is given a component, through an RF field for example, at right angles to the applied field $H_{0}$, this component will decay as [69]:

$$
\frac{d M_{x}}{d t}=-\frac{M_{x}}{T_{2}}, \frac{d M_{y}}{d t}=-\frac{M_{y}}{T_{2}}
$$

where $T_{2}$ is called the transverse relaxation time. Finally, in the presence of an applied field that is made of a static holding field and a much smaller RF field, the motion due to relaxation, as described above, can be added directly to the motion of a free spin [69]:

$$
\frac{d \vec{M}}{d t}=\gamma \vec{M} \times \vec{H}-\frac{M_{x} \hat{i}^{\prime}+M_{y} \hat{j}^{\prime}}{T_{2}}-\frac{\left(M_{z}-M_{0}\right) \hat{k}^{\prime}}{T_{1}}
$$

where $\hat{i}^{\prime}, \hat{j}^{\prime}$ and $k^{\prime}$ are the unit vectors in the laboratory from of reference.

An additional modification is necessary because $H_{1}$ in the polarized ${ }^{3} \mathrm{He}$ system is sufficiently large that the magnetizations relax to $\vec{M}_{0}=\chi_{0}\left(\vec{H}_{0}+\vec{H}_{1}(t)\right)$. This results in a set of modified Bloch equations [71]:

$$
\begin{aligned}
\frac{d M_{x}}{d t} & =-\frac{M_{x}-\chi_{0} H_{1}}{T_{2}}+\Delta \omega M_{y} \\
\frac{d M_{y}}{d t} & =-\Delta \omega M_{x}-\frac{1}{T_{2}} M_{y}-\omega_{1} M_{z} \\
\frac{d M_{z}}{d t} & =\omega_{1} M_{y}-\frac{M_{z}-M_{0}}{T_{1}}
\end{aligned}
$$

where $\Delta \omega=\gamma\left(H(t)-H_{0}\right)$ where $H_{0}=-\omega_{0} /$ gamma where $\omega_{0}$ is the Larmour frequency and $\omega_{1}=-\gamma H_{1}$.

This can be translated into a polarization vector $\vec{P}$ that can be written [71]:

$$
\frac{d P_{x}}{d t}(t)=-\frac{1}{T_{2}} P_{x}(t)+\gamma\left[H(t)-H_{0}\right] P_{y}(t)+\frac{1}{T_{2}} \chi H_{1}
$$




$$
\begin{aligned}
& \frac{d P_{y}}{d y}(t)=-\gamma\left[H(t)-H_{0}\right] P_{x}(t)-\frac{1}{T_{2}} P_{y}(t)+\gamma H_{1} P_{z}(t) \\
& \frac{d P_{z}}{d t}(t)=-\gamma H_{1} P_{y}(t)-\frac{1}{T_{1}} P_{z}(t)+\frac{1}{T_{1}} \chi H(t) .
\end{aligned}
$$

where $\chi=\mu / k_{B} T$ where $\mu$ is the magnetic moment of the nucleus, $k_{B}$ is the Boltzmann constant $(8.61739 \mathrm{e}-5 \mathrm{eV} / \mathrm{K})$ and $T$ is the temperature. These equations are used to calculate the shape of the NMR signal when the ${ }^{3} \mathrm{He}$ (or water for water calibrations) nuclei undergo AFP.

\subsubsection{Adiabatic Fast Passage}

Adiabatic Fast Passage is a way of reversing the polarization of nuclei in a magnetic field. This is done by applying a perpendicular RF field $H_{1}$ with frequency $\omega_{1}$ and increasing the field adiabatically until it goes through the Larmour resonance. At this resonance, the polarization direction of the nuclei will follow the effective field and change sign. Then the field is usually swept back to reverse the direction back to the original direction.

The speed at which the holding field is changed has to be slow enough to meet the adiabatic condition, but fast enough so sweeping is faster than the relaxation times $\left(T_{1}\right.$ and $\left.T_{2}\right)$. Polarized ${ }^{3} \mathrm{He}$ has $T_{1}$ of $435 \mathrm{~s}$ [57] making the adiabatic condition easily met with an $H_{1}=58 \mathrm{mG}, \omega_{1}=91 \mathrm{kHz}$ and a $H_{0}=25.0$ Gauss. Water has a $T_{1} \approx 3 s$ and therefore relaxes quickly under the same conditions; therefore, careful modeling of the relaxation is needed to extract the signal height.

The nominal holding field for the polarized ${ }^{3} \mathrm{He}$ target is 25 Gauss. The sweep rate for AFP is 1.2 Gauss/s. The field is swept up to 32 Gauss and then back down to 25 Gauss. A plot of the magnetic field $B$, the polarization along the holding field $\left(P_{z}\right)$ and perpendicular to the field $\left(P_{x}\right)$ during an polarized ${ }^{3} \mathrm{He}$ AFP sweep is shown in Fig. 6.22. 
$B_{z}$
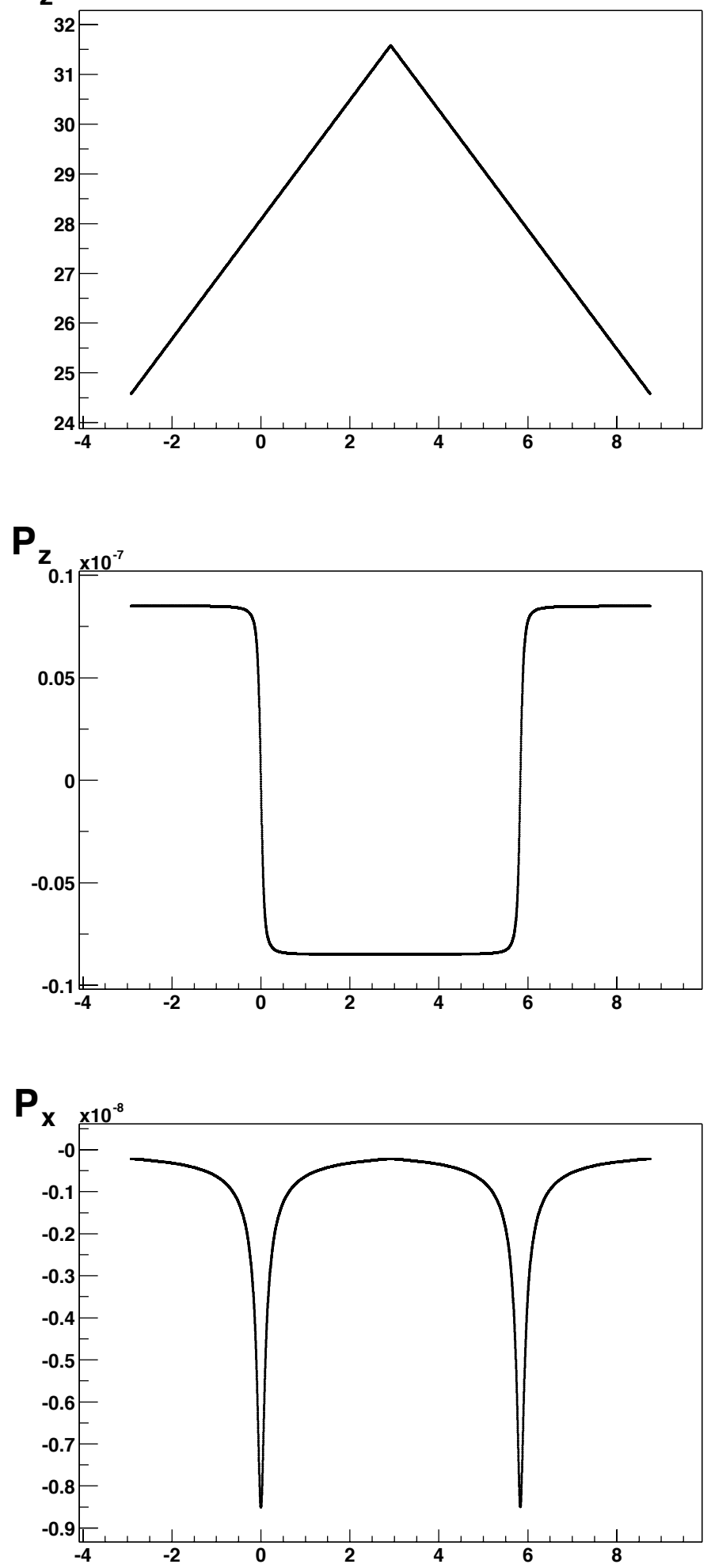

Figure 6.22: Adiabatic Fast Passage 


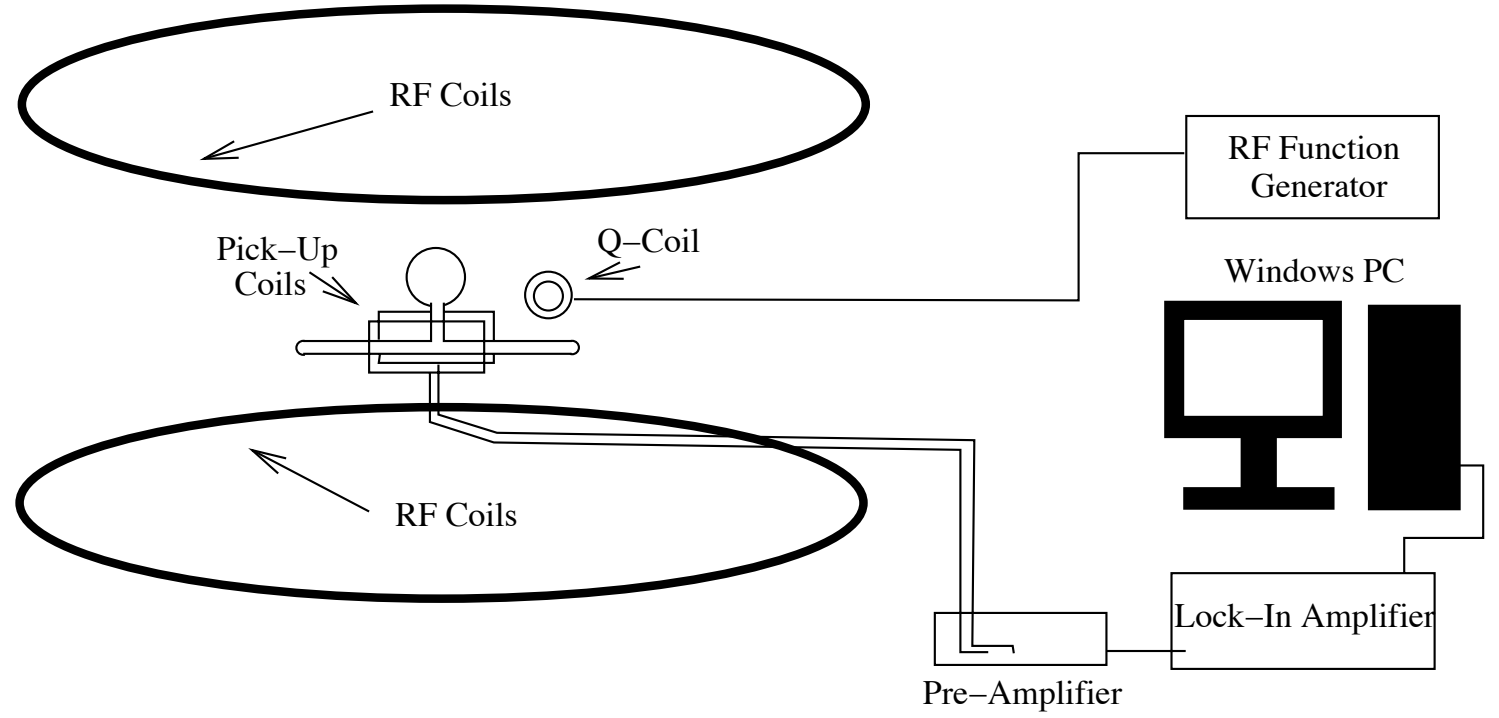

Figure 6.23: The equipment used to monitor NMR signal.

\subsubsection{NMR Polarimetry Setup}

The height of the transverse signal ( $P_{x}$ in Fig. 6.22) during AFP is proportional to the target polarization. This signal can be measured by a pair of coils (called "pick-up coils") placed near the target during AFP. In principle the polarization can be extracted directly from the signal height, but in practice it is better to calibrate with a known polarization. The known polarization used in the Jefferson Lab polarized ${ }^{3} \mathrm{He}$ target is the thermal polarization of water.

The NMR polarimetry system consists of three systems : the Helmholtz coils, the RF coils and the pick-up coils. The Helmholtz coils system and RF coils, described earlier in the chapter, work in tandem to perform AFP on the polarized ${ }^{3} \mathrm{He}$. The RF coils create the oscillating $H_{1}$ field, while the Helmholtz coils sweep the holding field through the Larmour resonance.

The pick-up coil system, shown in Fig. 6.23, detects the transverse oscillating magnetic field. The signal is amplified and filtered by a pre-amplifier. The signal is then sent to a lock-in amplifier that measures the $91 \mathrm{kHz}$ signal. The signal for the entire sweep is stored in the lock-in amplifier buffer then sent to a PC running 
LabView to be stored and analyzed.

The pick-up coils are simply two coils of 32 Gauge wire on plastic frames. The exact number of windings is not known, but it has been estimated by measuring the resistance of the coils to be about 96 . The shape of the pick-up coils is a $2 \mathrm{~cm}$ by $11 \mathrm{~cm}$ square. The coils on each side of the cell are wired in oppositely (if one is wound clock wise, the other is wound counterclockwise) so that the signal in the coils add and the background cancels.

Because the water signal is small, a lot of work goes into the placement of the coils. The pick-up coils are aligned so that a minimum amount of signal from the RF coils gets into the coils to maximize the sensitivity of the system. This is done by looking at the signal out of the pre-amplifier on oscilloscope and adjusting the pick-up coil frame with plastic shims and placement screws. Some background from the RF coils is needed for the lock-in amplifier, but generally the less RF signal the better.

\subsubsection{Extracting Polarization from the NMR Signal}

The shape of either peak when the holding field is being swept up through resonance (the "up sweep") or the signal when the holding field is being swept down through resonance (the "down sweep") can be written as the square root of a Lorentzian [57]:

$$
S(t)=\frac{h H_{1}}{\sqrt{H_{1}^{2}+\left(H(t)-H_{0}\right)^{2}}}+b H(t)+c
$$

where $S$ is the signal in the pick-up coils, $h$ is the signal height at resonance, $H_{0}$ is the value of the holding field at resonance and $t$ is time. $b$ and $c$ are parameters used to subtract background in the lock-in amplifier. The information about the 


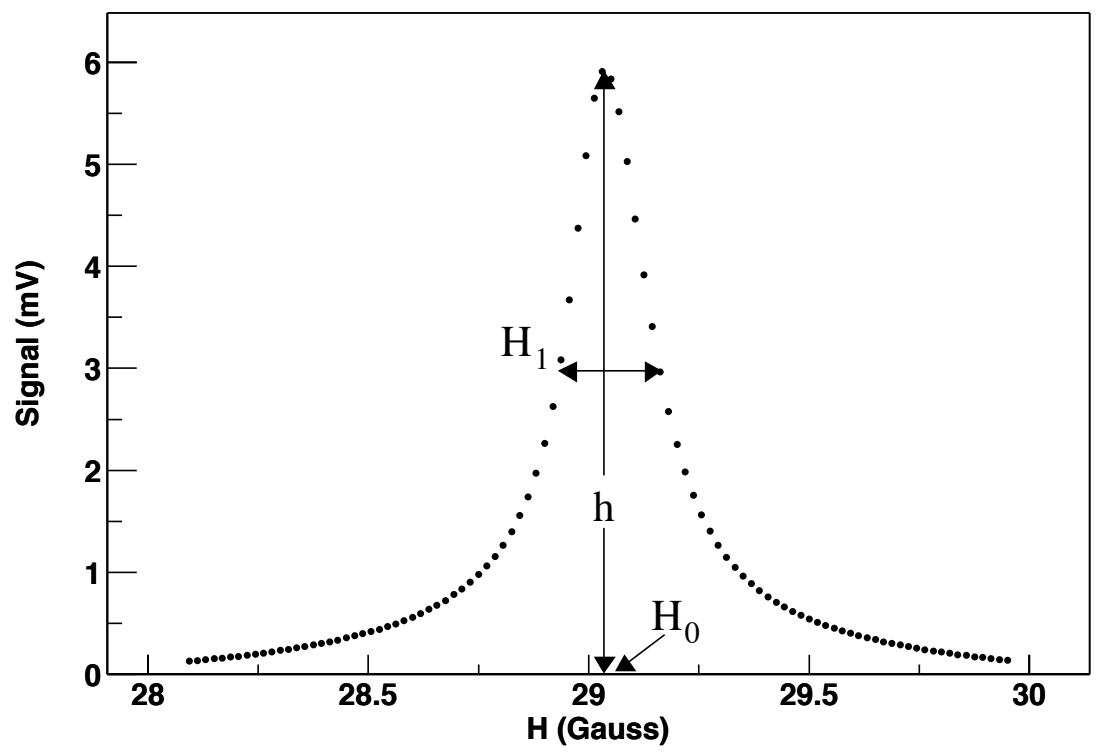

Figure 6.24: A plot of a signal from a typical NMR measurement. $h$ is the signal height, $H_{1}$ the amplitude of the $R F$ field and $H_{0}$ is the holding field value at the Larmour resonance.

polarization is contained in the value $h . H(t)$ for the polarized ${ }^{3} \mathrm{He}$ target is:

$$
H(t)= \begin{cases}\alpha t+\beta & \text { if } t<t_{\text {sweep }} \\ \beta-\alpha t & \text { if } t_{\text {sweep }}<t<2 t_{\text {sweep }}\end{cases}
$$

where $\alpha$ is the sweep rate (1.2 Gauss/s, in E97-103), $\beta$ is the initial holding field (25 Gauss in E97-103) and $t_{\text {sweep }}$ is the length of one sweep through resonance (5.8333 $\mathrm{s}$ in E97-103). Notice the $H t$ begins and ends at 25 Gauss with a maximum at 32 Gauss.

The polarization of the target can be extracted from $h$ using the formula [57]:

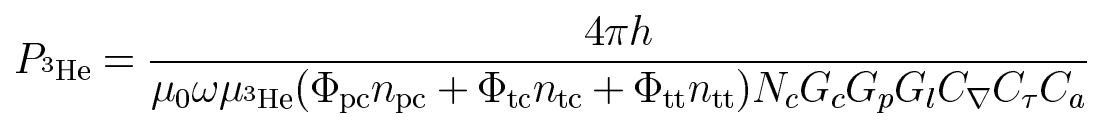

where $\mu_{0}$ is permeablity of free space and the rest of the parameters are given in Table 6.10. This is generally not done since there are unknown systematic associated with most of these parameters. No attempt was made to do this for E97-103.

The method used to extract the polarization from the ${ }^{3} \mathrm{He}$ NMR signal is to perform an NMR measurement for calibration using a sample of water. The water 


\begin{tabular}{cl}
\hline \hline Parameter & Description \\
\hline$h$ & NMR signal height of ${ }^{3} \mathrm{He}$ \\
$\mu_{3} \mathrm{He}$ & magnetic moment of ${ }^{3} \mathrm{He}\left(1.155 \times 10^{-13} \mathrm{MeV} / \mathrm{T}\right)$ \\
$\Phi_{\mathrm{pc}}$ & Magnetic flux of pumping chamber through pick-up coils \\
$n_{\mathrm{pc}}$ & ${ }^{3}$ He density in pumping chamber \\
$\Phi_{\mathrm{tc}}$ & Magnetic flux of target chamber through pick-up coils \\
$n_{\mathrm{tc}}$ & ${ }^{3}$ He density in target chamber \\
$\Phi_{\mathrm{tt}}$ & Magnetic flux of transfer tube through pick-up coils \\
$n_{\mathrm{tt}}$ & ${ }^{3}$ He density in transfer tube \\
$N_{c}$ & Number of windings in pick-up coils \\
$G_{c}$ & Gain of the pick-up coils \\
$G_{p}$ & Gain of the pre-amplifier \\
$G_{l}$ & Gain of the lock-in amplifier \\
$C_{\nabla}$ & Correction factor due to holding field gradients \\
$C_{\tau}$ & Correction factor due to lock-in time constant \\
$C_{a}$ & Correction factor due to anntenuation in cables \\
\hline \hline
\end{tabular}

Table 6.10: Parameters used in extract polarization from NMR signals.

\begin{tabular}{cl}
\hline \hline Parameter & Description \\
\hline$h_{w}$ & NMR signal height of water \\
$P_{t h}$ & Thermal polarization of water $(7.481 \mathrm{e}-9)$ \\
$\mu_{p}$ & magnetic moment of ${ }^{3} \mathrm{He}\left(8.795 \times 10^{-13} \mathrm{MeV} / \mathrm{T}\right)$ \\
$\Phi_{\mathrm{tot}}$ & Total magnetic flux of cell through the pick-up coils \\
$n_{\mathrm{tt}}$ & Density of protons in room temp water $(2482$ Amagats $)$ \\
$G_{p}^{w}$ & Gain of the pre-amplifier in water signal \\
$C_{\nabla}^{w}$ & Correction factor due to holding field gradients in water signal \\
$C_{\tau}^{w}$ & Correction factor due to lock-in time constant in water signal \\
\hline \hline
\end{tabular}

Table 6.11: Additional parameters used in water calibrated NMR

sample used for this experiment was containted in a glass cell made as similar in dimensions to the helium cells as possible. The protons in water have a magnetic moment that can be aligned in a magnetic field which give the water a small, but well-known polarization. This polarization can be described by $P_{w}=(\chi H)$ where $\chi=3.4616 \times 10^{-10} /$ Gauss at $22{ }^{\circ} \mathrm{C}[71]$. The gyro-magnetic ratio for the proton is $2.67515 \times 10^{4} /($ Gauss s) therefore the resonance peak with a frequency of $91 \mathrm{kHz}$ is 21.37 Gauss [71].

The polarization of the NMR can be extracted from a ratio of Eq. 6.58 for ${ }^{3} \mathrm{He}$ 


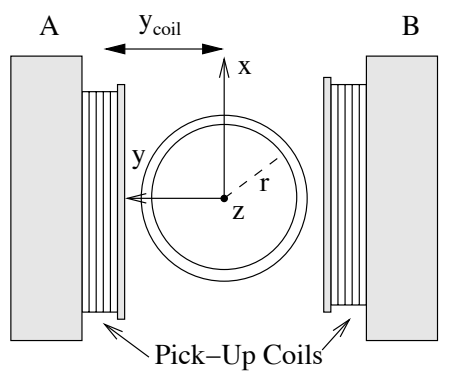

Front View

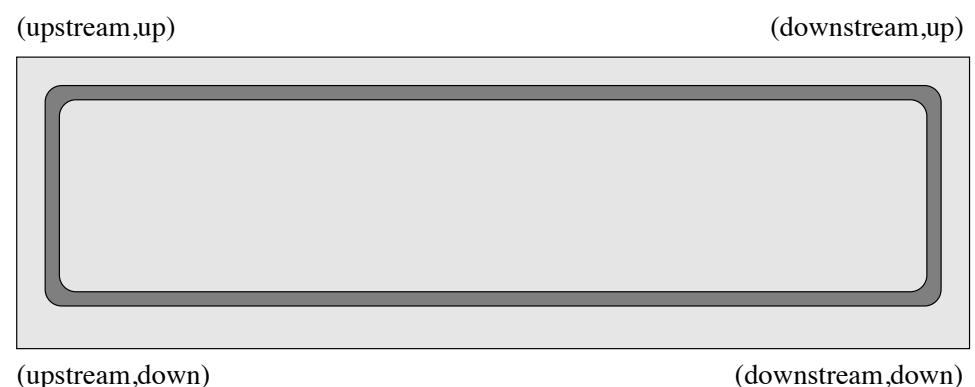

Side View

Figure 6.25: The reference frame of the magnetic flux calculation with respect to the target chamber and the pick-up coils.

and water:

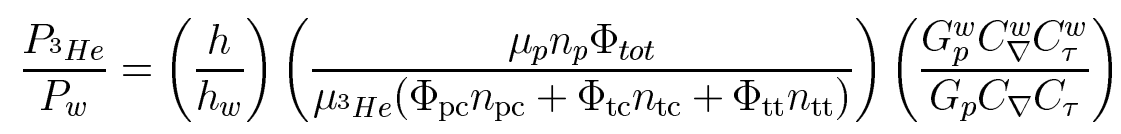

where the individual parameters are listed in tables 6.10 and 6.11. Notice that some of the parameters cancel immediately. In most cases $C_{\nabla}$ and $C_{\tau}$ also cancel, but are kept in here because of special cases that occurred during the experiment. Since $P_{w}$ is known, after measurement of the water signal $h_{w}$ a calibration constant $c_{w}$ is calculated :

$$
c_{w}=\left(\frac{P_{w}}{h_{w}}\right)\left(\frac{\mu_{p} n_{p} \Phi_{t o t}}{\mu_{3} H e\left(\Phi_{\mathrm{pc}} n_{\mathrm{pc}}+\Phi_{\mathrm{tc}} n_{\mathrm{tc}}+\Phi_{\mathrm{tt}} n_{\mathrm{tt}}\right)}\right)\left(\frac{G_{p}^{w} C_{\nabla}^{w} C_{\tau}^{w}}{G_{p} C_{\nabla} C_{\tau}}\right)
$$

then the polarization from a particular NMR polarization measurement on ${ }^{3} \mathrm{He}$ can be extracted with $P_{3_{H e}}=c_{w} h$. Each of the parameters that go into $c_{w}$ will be analyzed in the following subsections.

\subsubsection{NMR Flux in the Pick-up Coils}

The magnetic flux is the amount of magnetic field normal to an area. In the context of the NMR polarimetry the field is from the ${ }^{3} \mathrm{He}$ nuclei or protons in water going through resonance and the area is the face of the pick-up coils. The amount of current in the coils is proportional to the flux. The magnetic flux is written:

$$
\Phi=\int \frac{\vec{B}}{B} \cdot d \vec{a}=\oint_{\text {coils }} \frac{\vec{A}}{A} \cdot d \vec{l}
$$




\begin{tabular}{|c|c|c|c|c|c|c|}
\hline & \multicolumn{3}{|c|}{ Shapiro } & \multicolumn{3}{|c|}{ Virginia One } \\
\hline Corner Name & $\mathrm{x}$ & $\mathrm{y}$ & $\mathrm{Z}$ & $\mathrm{x}$ & $\mathrm{y}$ & $\mathrm{Z}$ \\
\hline A, Down, Downstream & -1.023 & 1.567 & 5.510 & -0.961 & 1.658 & 5.510 \\
\hline A, Up, Downstream & 0.977 & 1.568 & 5.510 & 1.039 & 1.660 & 5.510 \\
\hline A, Up, Upstream & 0.977 & 1.506 & -5.510 & 1.039 & 1.585 & -5.510 \\
\hline A, Down, Upstream & -1.023 & 1.502 & -5.510 & -0.961 & 1.578 & -5.510 \\
\hline B, Down, Downstream & -1.023 & -1.904 & 5.510 & -0.961 & -1.819 & 5.510 \\
\hline B, Up, Downstream & 0.977 & -1.908 & 5.510 & 1.039 & -1.822 & 5.510 \\
\hline B, Up, Upstream & 0.977 & -1.803 & -5.510 & 1.039 & -1.731 & -5.510 \\
\hline B, Down, Upstream & -1.023 & -1.798 & -5.510 & -0.961 & -1.720 & -5.510 \\
\hline \multicolumn{7}{|l|}{ Cell Dimension } \\
\hline Pumping Chamber Radius & & 3.03 & & & 2.98 & \\
\hline Transfer Tube Radius & & 0.398 & & & 0.396 & \\
\hline Transfer Tube Length & & 6.46 & & & 6.52 & \\
\hline Target Chamber Radius & & 0.796 & & & 0.819 & \\
\hline Target Chamber Length & & 40.0 & & & 39.4 & \\
\hline
\end{tabular}

Table 6.12: Values used for the flux calculation. All values are in centimeters

where $\vec{B}$ is the magnetic field from the nuclei and $\vec{A}$ is the magnetic vector potential. The definition of flux here is normalized $(\vec{B} / B$ instead of just $\vec{B})$ to unit magnetization so that the flux is independent of the density and polarization of the cell, which enter the calibration constant independently. Using the reference frame defined by Fig. 6.25, the magnetic vector potential is:

$$
\vec{A}(\vec{r})=\int_{V_{\text {cell }}} d^{3} \vec{r} \frac{-\hat{y} \times \vec{r}}{|\vec{r}|^{3}}
$$

where $V_{\text {cell }}$ is the volume of the cell and $-\hat{y}$ is a unit vector in the $-\mathrm{y}$ direction representing the direction of magnetization.

The code used to calculate the magnetic flux was written by Ioannis Kominis [61] divides the cell into small cubes of equal volume. $\vec{A}(\vec{r})$ at any $\vec{r}$ is the sum of the vector potential of all of these cubes. It then sums $\vec{A}(\vec{r}) \cdot d \vec{l}$ over the path of one of the pick-up coils. The target chamber, transfer tube and pumping chamber fluxes are calculated separately for both pick-up coils.

The results of these calculations are shown for the two cells in Table 6.13 using the cell and pick-up coil geometries in Table 6.12. The internal radius of the target 


\begin{tabular}{|c|ccccc|}
\hline \hline Cell & Coil & $\begin{array}{c}\text { Target } \\
\text { Chamber }\end{array}$ & $\begin{array}{c}\text { Pumping } \\
\text { Chamber }\end{array}$ & $\begin{array}{c}\text { Transfer } \\
\text { Tube }\end{array}$ & Total \\
\hline \multirow{3}{*}{ Shapiro } & $\mathrm{A}$ & -25.15 & 1.84 & 0.04 & -23.26 \\
& $\mathrm{~B}$ & 19.12 & -1.76 & 0.05 & 17.41 \\
& $\mathrm{~B}-\mathrm{A}$ & 44.26 & -3.59 & 0.01 & 40.69 \\
\hline & $\mathrm{A}$ & -24.78 & 1.74 & -0.01 & -23.04 \\
Virginia & $\mathrm{B}$ & 21.63 & -1.70 & 0.05 & 19.97 \\
One & $\mathrm{B}-\mathrm{A}$ & 46.41 & -3.45 & 0.05 & 43.01 \\
\hline \hline
\end{tabular}

Table 6.13: Flux values for polarized ${ }^{3}$ He cells. All values are in $\mathrm{cm}^{2}$.

chamber is calculated by subtracting the wall thickness from the external radius for the middle section of the cell (where the pick-up coils are). The pumping chamber volume is derived by using the internal volume and assuming it is spherical. The transfer tube internal radius is determined by assuming it is a cylinder. The placement of the pick-up coils assumes that the cell axis is along $\mathrm{z}=0 \mathrm{~cm}$ and the center of the cell is at $\mathrm{x}=0 \mathrm{~cm}$ and $\mathrm{y}=0 \mathrm{~cm}$. The position of the pick-up coil corners are determined by measuring the distance between the corners with the cell out of the pick-up coils and then the cell is inserted and its position is determined with a caliper.

The error caused by measurement of the cell position is a major systematic error in NMR. The effect of mismeasurements on the total cell flux of a cell is shown in Fig. 6.26. These distributions were determined by running the flux calculation code 100 times while varying a set of parameters randomly within the error bars of the measurement. The uncertainties in measurements were chosen by looking at the distribution of measurement (for instance the space between the top of the pick-up coils) repeated several times. The overall error in the flux due to mismeasurement is $2.0 \%$.

A study was done to see how well the flux calculation used agreed with an absolute flux number. This was done by taking NMR measurements from a polarized ${ }^{3} \mathrm{He}$ cell with the pick-up coils in the same position, but moving the cell slowly 

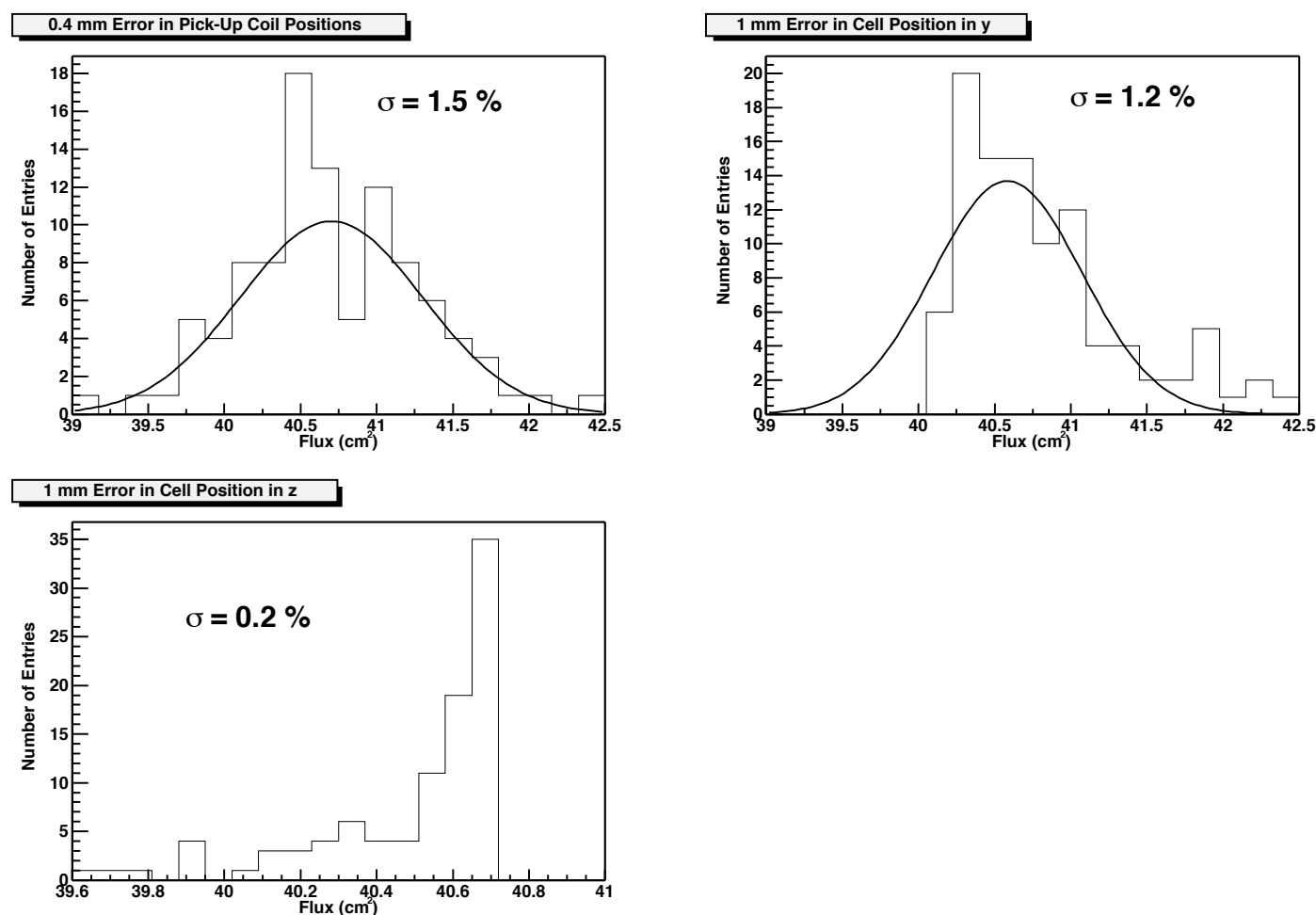

Figure 6.26: The reference frame of the magnetic flux calculation with respect to the target chamber and the pick-up coils.

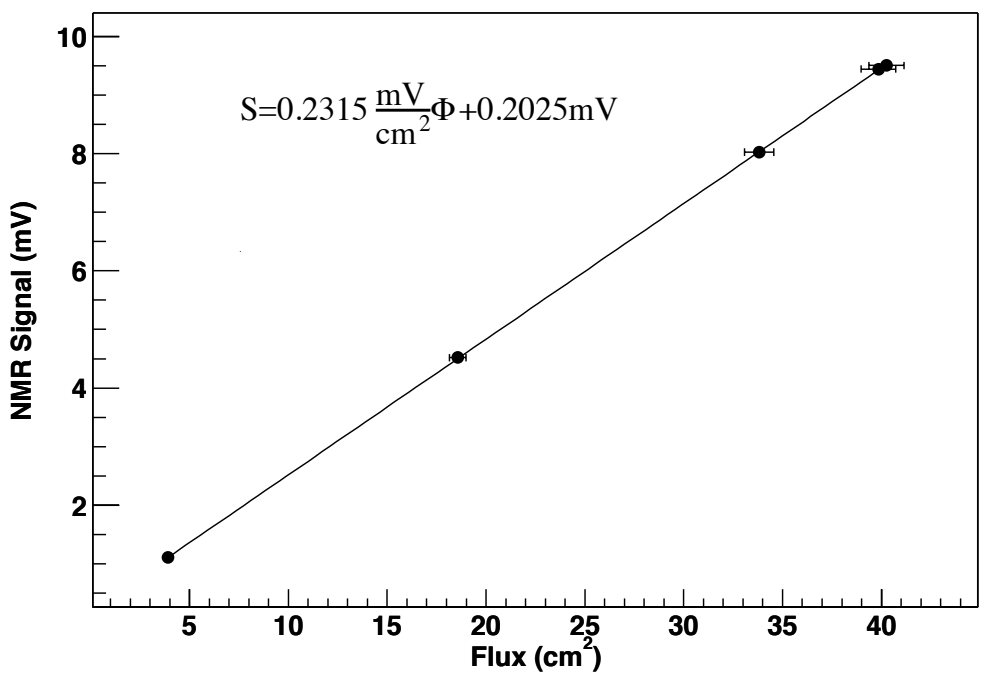

Figure 6.27: A plot of the helium signal height vs. flux. 


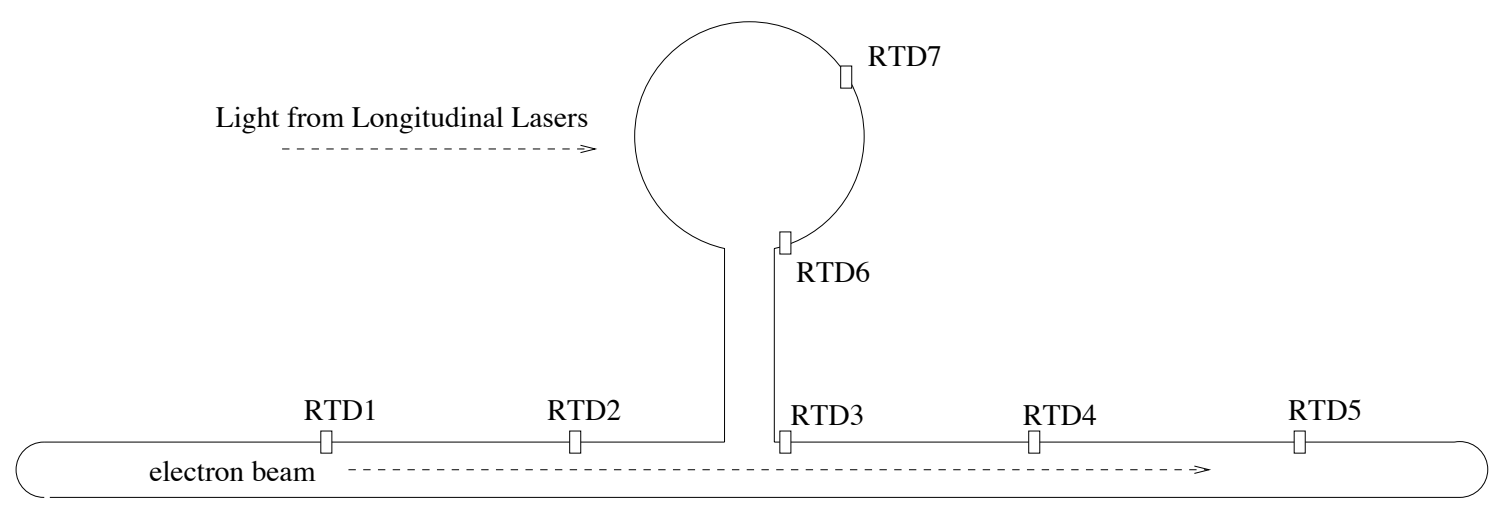

Figure 6.28: The placement of the temperature sensors in E97-103.

upwards. Since the vertical motion on the target is done with a precision mechanical lifter it can be done very precisely. The goal was to change the flux and see if the model followed the expected trend. In this case, the ratio of flux to signal should be constant and when the signal is zero the flux should be zero. A plot of the data taken is shown in Fig. 6.27.

The signal does to seem be clearly linear, but the $y$-intercept is non-zero. The shift, while large at low flux, is about $2 \%$ of the flux at the place where the cell usually sits. The source of this shift is unknown, thought there are a number of factors that could contribute to it. There could be a calculation error in the flux calculation code. Another reason is that error bars on the low flux points are being underestimated. Using an absolute error of $0.85 \mathrm{~cm}^{2}$ on all the data points allows the a line going through zero to be fit with a $\chi^{2}=1$. In both cases, an absolute shift effects the small flux data more than the high flux data. Therefore, an additional error of $1.3 \%$ of systematic error was added to the $2.0 \%$ of measurement error to the flux. This allows one to plot the points with a flux $>15 \mathrm{~cm}^{2}$ with a line forced to go through zero and a $\chi^{2}=1$. This should be a reasonable amount of uncertainty for this effect in these measurements. 


\subsubsection{Measuring Cell Temperature and Density}

Using the ideal gas law, the number density of the target chamber during an NMR measurement is determined by :

$$
n_{\mathrm{tc}}=n_{0}\left[1+\frac{V_{\mathrm{pc}}}{V_{\mathrm{tot}}}\left(\frac{T_{\mathrm{tc}}}{T_{\mathrm{pc}}}-1\right)\right]^{-1}
$$

where $n_{0}$ is the room temperature number density of the cell, $V_{\mathrm{pc}}$ is the internal volume of the cell pumping chamber, $V_{\text {tot }}$ is the total internal volume of the cell, $T_{\mathrm{tc}}$ is the average target chamber temperature and $T_{\mathrm{pc}}$ is the average temperature of pumping chamber. Similarly, the number density of the pumping chamber can be calculated:

$$
n_{\mathrm{pc}}=n_{0}\left[1+\frac{V_{\mathrm{tc}}}{V_{\mathrm{tot}}}\left(\frac{T_{\mathrm{pc}}}{T_{\mathrm{tc}}}-1\right)\right]^{-1}
$$

where $V_{\mathrm{tc}}$ is the internal volume of the target chamber.

The cell temperature is measured with a series of high-temperature resistive thermal diodes (RTDs) made by Omega. The RTD placement is shown in Fig. 6.28. The target chamber temperature, $T_{\mathrm{tc}}$, was calculated by simply taking an average of RTDs 1-5. More sophisticated analysis yielded average temperatures within $1 \%$ of this temperature. This is because the temperatures were more or less the same along the target chamber. Normally, because the pumping chamber is at a much higher temperature, RTD3 is $20^{\circ} \mathrm{C}$ higher than the RTD1 and RTD5. In the case of E97-103, there were helium jets blowing on the ends of the cells (for protecting the cell from ruptures) and that made the thermal gradient smaller in the target chamber, where RTD3 $<5.0^{\circ} \mathrm{C}$ different from RTD1 and RTD5.

The pumping chamber also has RTDs on it; however, these RTDs only reflect the interior temperature of the cell when the lasers are off. When the lasers on, there is a large thermal gradient between the inner part of the cell and the edge of the pumping chamber. To measure the average pumping chamber temperature, a separate measurement using the NMR system is made. 
This measurement compares the NMR signal height when the lasers are on and when the lasers are off. The procedure is to take an NMR measurement and record the RTD temperatures with the lasers on and the temperature stable. Then the lasers are turned off and when the temperature stabilizes (about 15-20 minutes) take another NMR and record the RTD temperature. The lasers are then turned back on and when the temperature stabilizes (another 15-20 minutes) another NMR measurement is done and the RTD temperatures are recorded. The NMR signal height from the two NMR measurements done with the lasers are on are averaged as well as their temperatures. Then the following formula can be used to calculate the average pumping chamber temperature:

$$
T_{\mathrm{pc}}=V_{\mathrm{pc}} T_{\mathrm{tc}}^{\text {on }}\left\{\frac{S^{\text {off }}}{S^{\text {on }}} \frac{T_{\mathrm{tc}}^{\mathrm{on}}}{T_{\mathrm{tc}}^{\mathrm{off}}} \frac{T_{\mathrm{cooils}}^{\text {off }}}{T_{\mathrm{coils}}^{\text {on }}}\left[V_{\mathrm{tot}}+V_{\mathrm{pc}}\left(\frac{T_{\mathrm{tc}}^{\mathrm{on}}}{T_{\mathrm{pc}}^{\text {off }}}-1\right)\right]-V_{\mathrm{tot}}+V_{\mathrm{pc}}\right\}^{-1}
$$

where $T_{\mathrm{tc}}^{\mathrm{on}}$ and $T_{\mathrm{tc}}^{\mathrm{off}}$ is the temperature of the target chamber when the lasers are on and off respectively, $S^{o n}$ and $S^{o f f}$ is the height of the NMR signal with the lasers on and off respectively and $T_{\text {coils }}^{o n}$ and $T_{\text {coils }}^{o f f}$ is the temperature of the part of the target chamber between the pick-up coils. $T_{\text {coils }}$ can be calculated by :

$$
T_{\text {coils }}=\frac{1}{2}\left[T_{3}+\frac{1}{2}\left(T_{2}+T_{4}\right)\right]
$$

where $T_{2}, T_{3}$ and $T_{4}$ are the temperatures from RTD 3,4 and 5 respectively.

Once this measurement has been done several times, the internal temperature versus the external temperature can be plotted, as it has been in Fig. 6.30. The measurements have been separated for the two cells as the exterior RTDs had different placement and different levels of thermal contact with the cell. This data is from specific temperature measurements. To calculate $T_{\mathrm{pc}}$ for a specific NMR measurement the values of RTD6 and RTD7 and a fit to this data were used to calculate the internal pumping chamber density. The calibrations used are:

$$
T_{\mathrm{pc}}^{\text {Shapiro }}=1.464\left(T_{6}+T_{7}\right)-838.7
$$




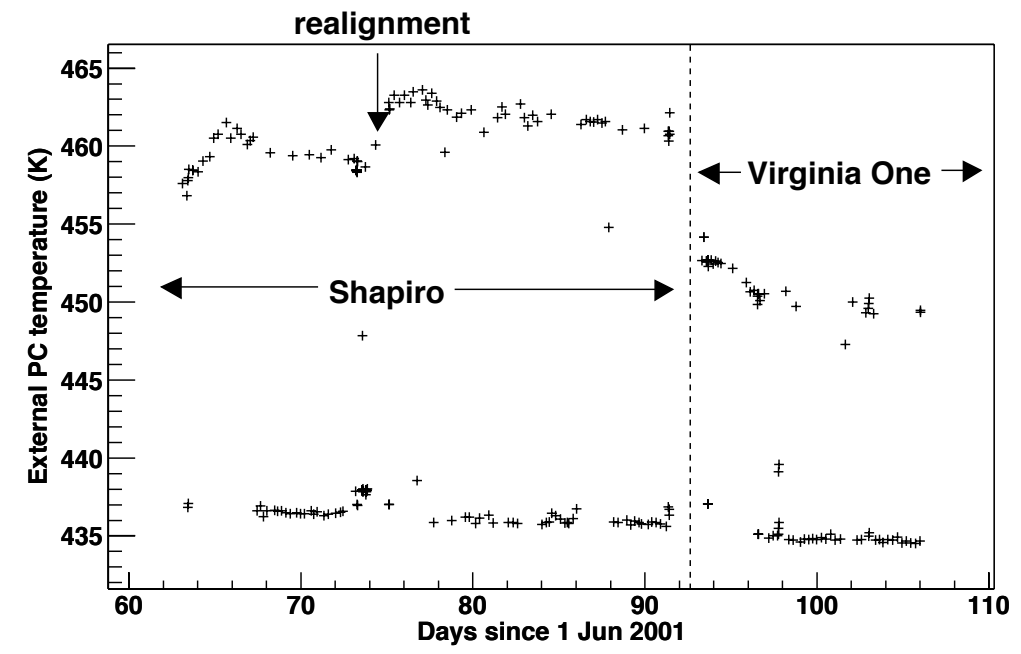

Figure 6.29: The external pumping chamber temperatures during NMR measurements in E97-103.
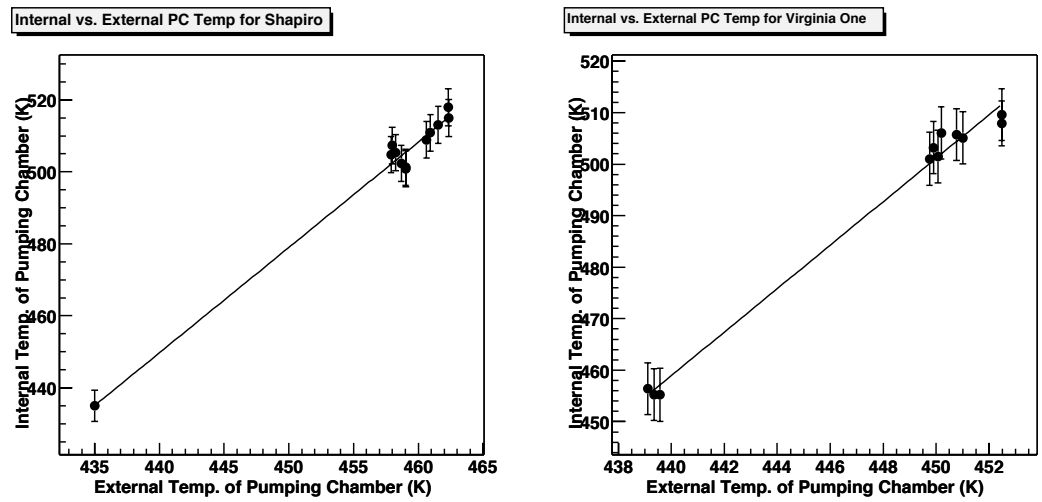

Figure 6.30: The relationship between the average reading of RTD6 and RTD7 and the average internal temperature of the pumping chamber. 


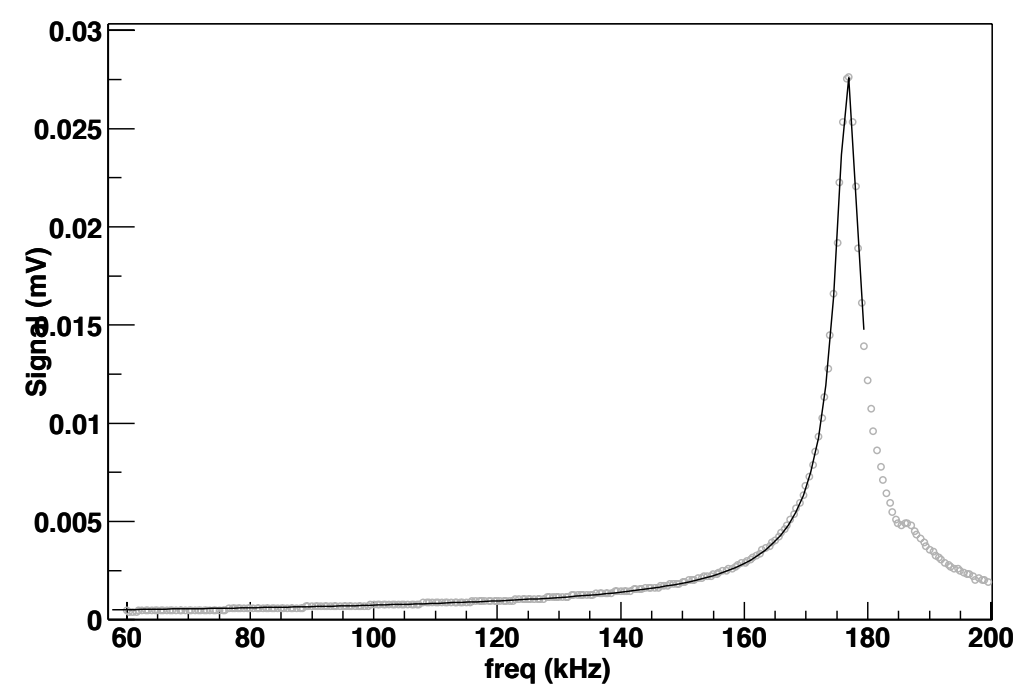

Figure 6.31: An example of a resonance curve created by the $Q$-coil.

$$
T_{\mathrm{pc}}^{\text {VirginiaOne }}=2.111\left(T_{6}+T_{7}\right)-1398.3
$$

where $T_{6}$ and $T_{7}$ are the temperatures from RTD 6 and RTD 7. The uncertainty in this measurements was estimated to be $8^{\circ} \mathrm{C}$.

Its useful to know the average density when running taking production data. The average density of Shapiro with the three lasers on was 10.72 Amagats. The average density of Virginia One with three lasers on was 10.01 Amagats.

\subsubsection{The Gain of the Pick-Up Coils}

The gain of the pick-up coils is monitored throughout the experiment. This is done by a small coil of wire, known as the q-coil, that is glued to the side of the target chamber with the magnetic field of the q-coil pointing at the pick-up coils. The coil is connected directly to the RF function generator as shown in Fig. 6.23. The coil is run slowly through a series of frequencies from $65 \mathrm{kHz}$ to $300 \mathrm{kHz}$ at a constant amplitude. This measures the relative pick-up coil response as a function of frequency. 


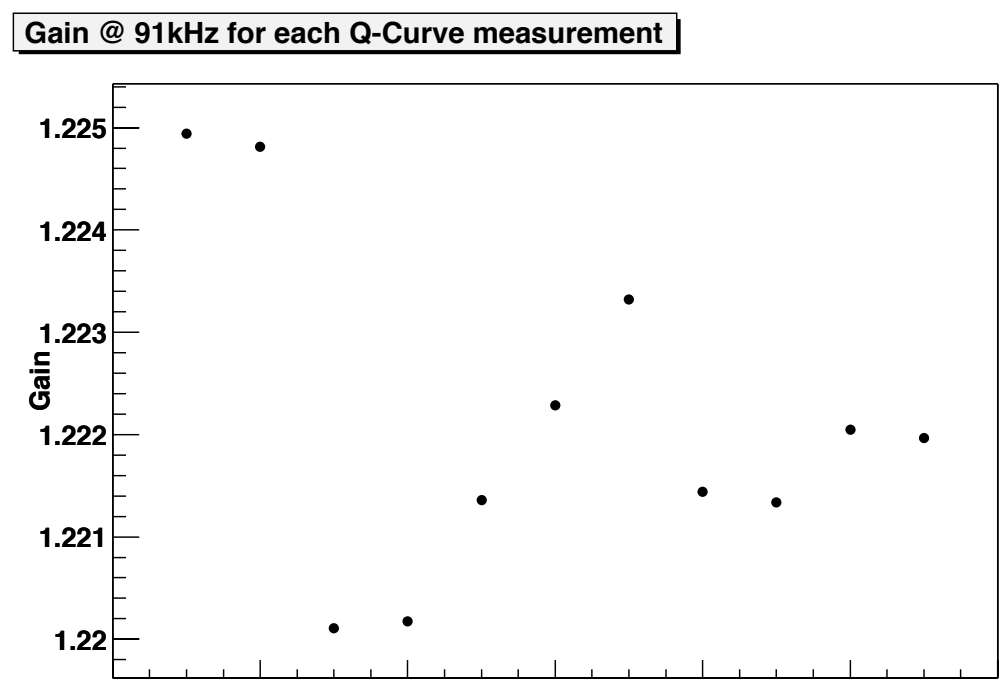

Figure 6.32: Measures of the gain throughout the experiment.

The data from this measurement can be fit with [72]:

$$
V(f)=\frac{k f}{\sqrt{\frac{1}{Q^{2}} \frac{f^{2}}{f_{0}^{2}}+\left(\frac{f^{2}}{f_{0}^{2}}-1\right)^{2}}}+c
$$

where $V(f)$ is the voltage output of the pick-up coils, $f$ is the frequency of the q-coil and $k, Q, f_{0}$ and $c$ are fitted parameters. The gain can be extracted for any frequency once these parameters are known:

$$
G(f)=\frac{1}{\sqrt{\frac{1}{Q^{2}} \frac{f^{2}}{f_{0}^{2}}+\left(\frac{f^{2}}{f_{0}^{2}}-1\right)^{2}}}
$$

where $G(f)$ is the gain of the circuit. The gain for the frequency $91 \mathrm{kHz}$ is presented in Fig. 6.32. There is a less than $0.3 \%$ deviation in gain throughout the experiment.

\subsubsection{Modifications to the NMR signal shape}

There are two important modifications to the NMR signal shape. The first is the time constant from the lock-in amplifier that reads in the voltage from the pickup coils. The time constant tells the lock-in how long to integrate the data before outputting a value. Increasing the time constant suppresses noise, but distorts the 


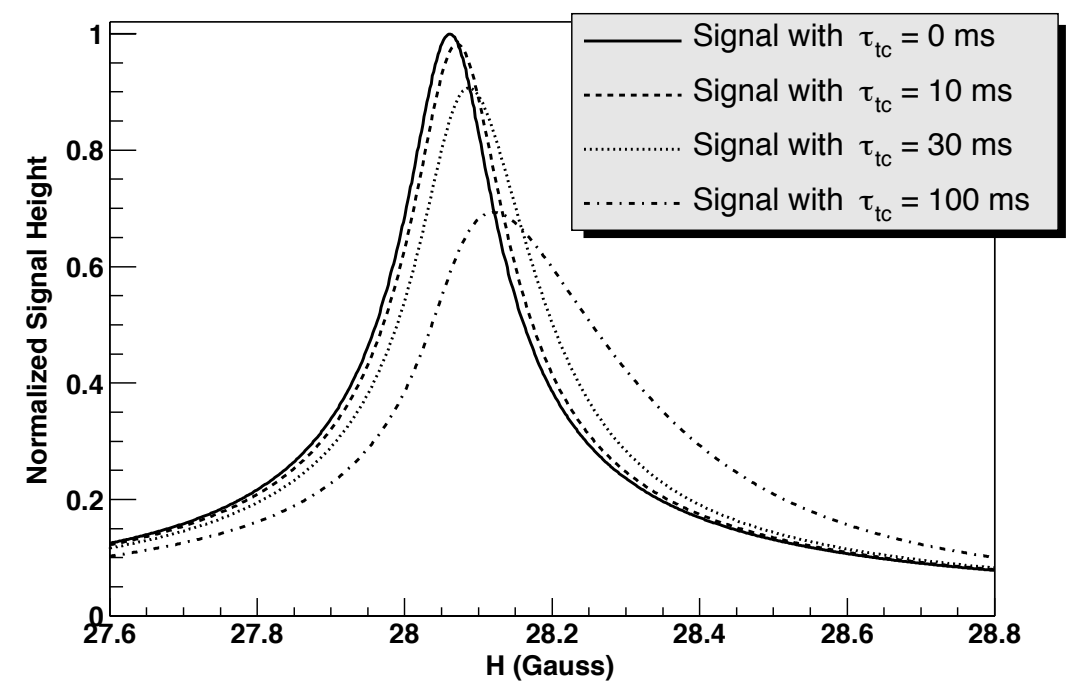

Figure 6.33: The effect of the lock-in amplifier time constant on a normalized NMR signal with an $H_{1}$ of $58 \mathrm{mG}$.

signal. Fortunately, the signal distortion is predictable. The differential equation representing the effect of the lock-in time constant on the NMR signal is [73]:

$$
\frac{d S_{\text {lock-in }}(t)}{d t}=\frac{1}{\tau_{\text {tc }}}\left(S_{\text {raw }}(t)-S_{\text {lock-in }}(t)\right)
$$

with initial condition:

$$
\frac{d S_{\text {lock }- \text { in }}(0)}{d t}=0.0
$$

where $S_{\text {lock-in }}$ is the signal in the lock-in, $S_{\text {raw }}$ is the raw signal out of the pick-up coils and $\tau_{\text {tc }}$ is the lock-in time constant. The lock-in time constant used for this experiment was $30 \mathrm{~ms}$. It was chosen to reduce noise in the water signal, but not to be so large as to distort the signal significantly.

The second source of distortion is from holding field gradients. The gradients distort the signal because different parts of the cell will resonate at different times with the result being broadening of the signal. These became especially critical to analyzing the helium signal for Virginia One since its data was taken with a set of coils intentional causing a gradient. (These coils were used to stop the masing effect 


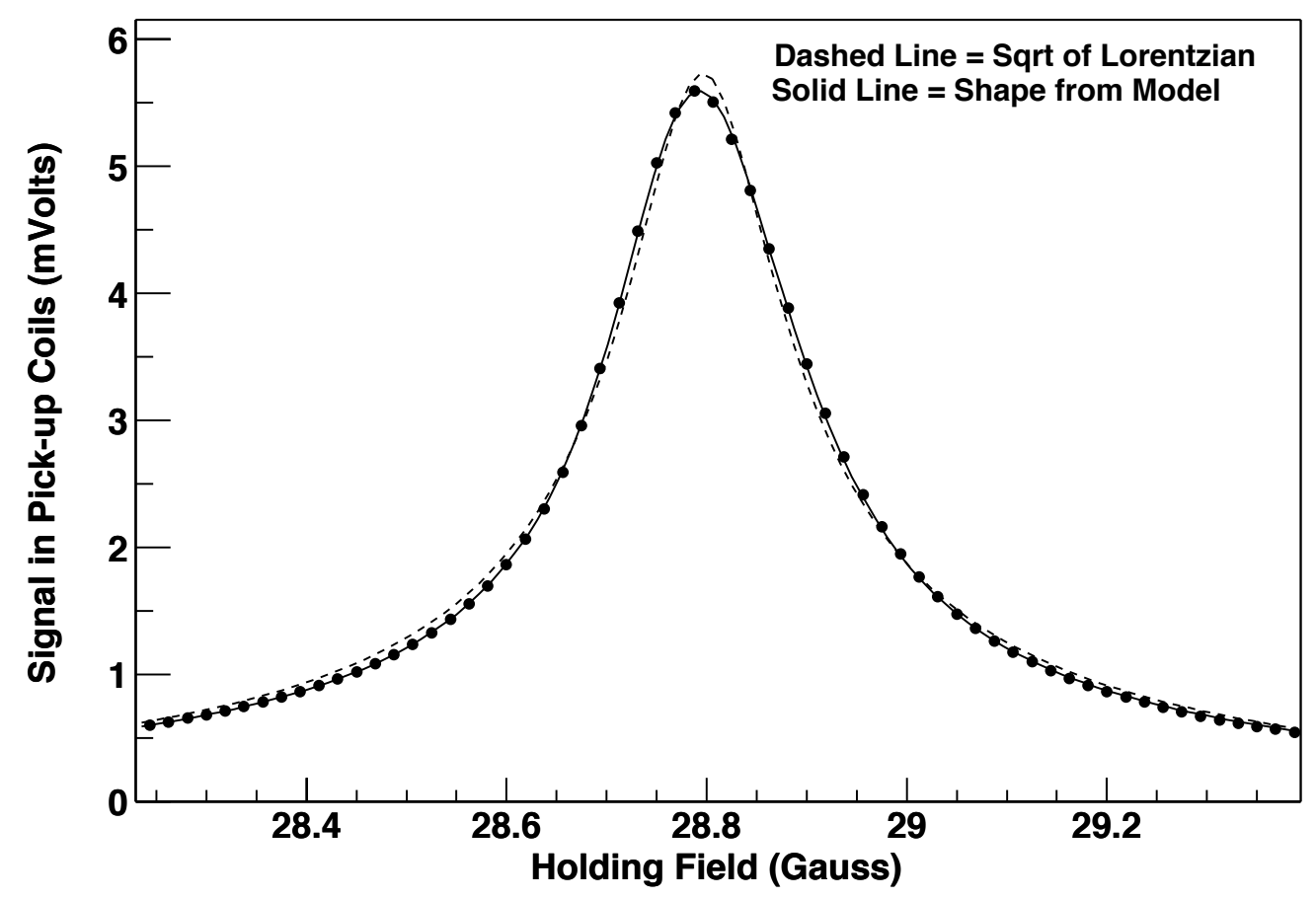

Figure 6.34: NMR data fit two different ways : a square root of a Lorentzian and one with a shape corrected for time-constant effect and holding field gradients.

described later). The signal can be modeled numerically using the formula:

$$
S_{\text {corr }}(H)=\frac{1}{L \Phi_{\text {tot }}} \sum_{i=0}^{N} \Phi(i \Delta z-L / 2) \Delta z S_{\mathrm{tc}}\left(H+\frac{d B_{z}}{d z}(i \Delta z-L / 2)\right)
$$

where $S_{\text {corr }}$ is the corrected signal, $L$ is the length of the cell, $\Phi_{\text {tot }}$ is the total flux from the target chamber, $N$ is the number of divisions of the target chamber in the calculation, $\Delta z$ is $L / N, \Phi(z)$ is the amount of flux for a section $\Delta z$ of the target chamber, $S_{\mathrm{tc}}(H)$ is the NMR signal corrected for the lock-in time constant and $d B_{z} / d z$ is the field gradient along the target chamber axis. $\Phi(z)$ was calculated with the flux code and is shown in Fig. 6.35.

The procedure for creating a model of the signal shape for NMR in E97-103 was to first numerically integrate Eq. 6.71 with a $S_{\text {raw }}$ from Eq. 6.56 using a 4 th order Runge-Kutta from Numerical Recipes in $C$ [74]. The signal is broken into sections along $H$ and the mean value of the signal for the section is placed in an array. Then 


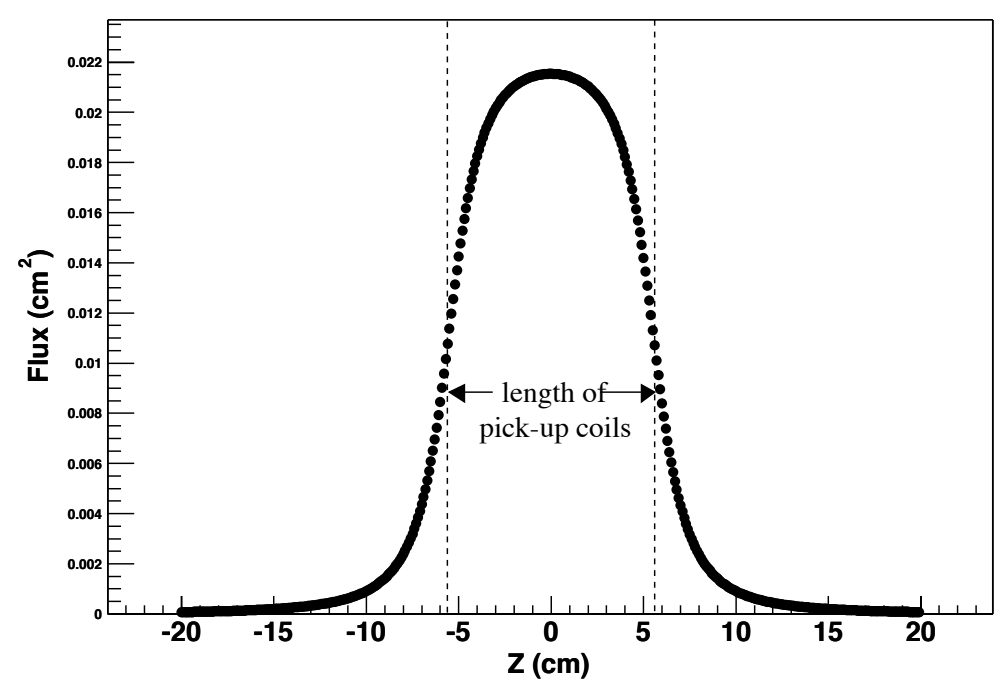

Figure 6.35: The amount of flux from a section of target chamber as a function of $z$.

the signal is summed by using the elements of the array. Examples of signals with gradients are shown in Fig. 6.36.

The models of the signal shape are used to calculate correction factors to the signal. While the model works well for the lock-in time constant and for smaller gradients, it doesn't work especially well for signal taken under high gradients. High gradient fields only occurred when a set of coils, known as gradient coils, were used to intentionally create gradients to break up the masing effect. No gradient coils were used during Shapiro's running period, but the gradient coils were used throughout Virginia One's running period.

The gradient coils were placed parallel to both sets of Helmholtz coils. The set on the small coils (along the longitudinal direction) were twenty windings of 18 gauge wire. The set on the large coils (along the transverse direction) were 10 windings of 10 gauge wire. They were powered by a power supply could deliver a maximum of 7 Amps of current. Virginia One used them with 2 Amps on the longitudinal gradient coils and 7 Amps on the transverse gradient coils. The correction to the NMR signal from these gradient coils were measured empirically. After the experiment 


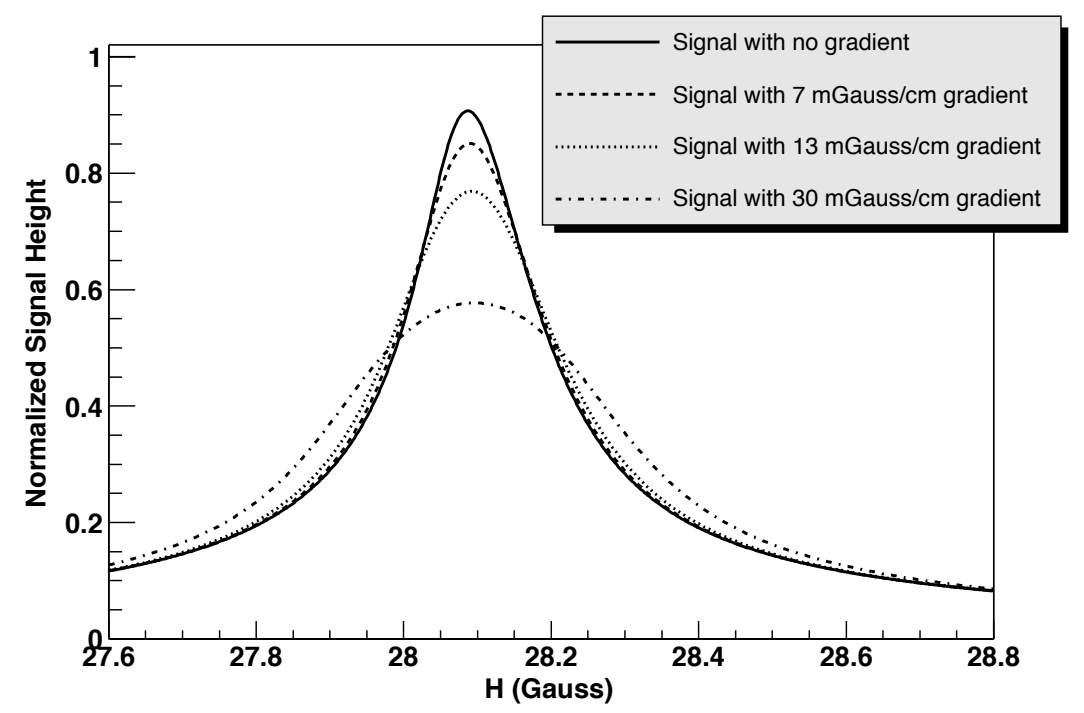

Figure 6.36: Examples of different sizes of gradients on the ${ }^{3} \mathrm{He}$ NMR signal.

was finished a study was done to compare the NMR signal with and without the gradient coils. To do this a NMR signal was taken with no power in the gradient coils. The gradient coil was turned up to a certain voltage. Another NMR was taken. The gradient coil was turned off again and another NMR was taken. This was done for the entire range of currents for both the longitudinal and transverse gradient coils.

All the signals in this study were fit with Eq. 6.56. The ratio of the heights of the signal when the gradient coils were on to the average heights of the two signals around it that had the gradient coils off are plotted in Fig. 6.37 and Fig. 6.38. The transverse gradient coils had almost no effect on the NMR signal.

\subsubsection{Polarization Loss due to AFP measurements}

Small polarization losses are inevitable during AFP measurements. The AFP loss is measured by simply taking several NMR measurements back-to-back. Usually, a time span of 3-5 minutes between measurements are done, though this may not be necessary. The average loss per measurement is calculated assuming no other 


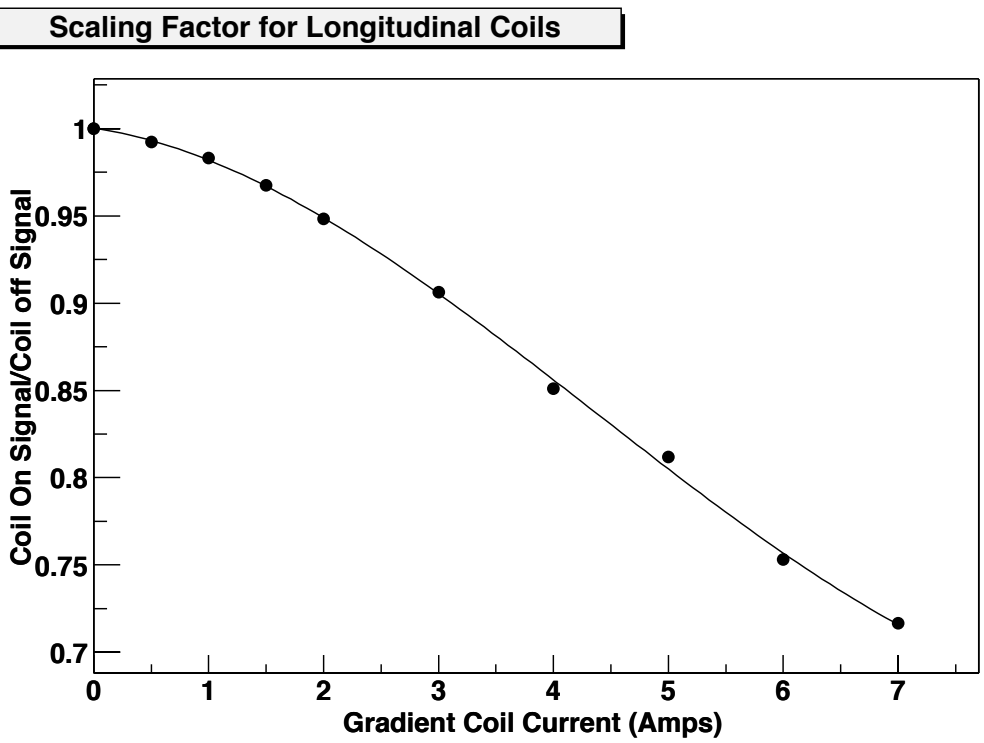

Figure 6.37: The effect on NMR signal height of the longitudinal gradient coil.

\section{Scaling Factor for Transverse Coils}

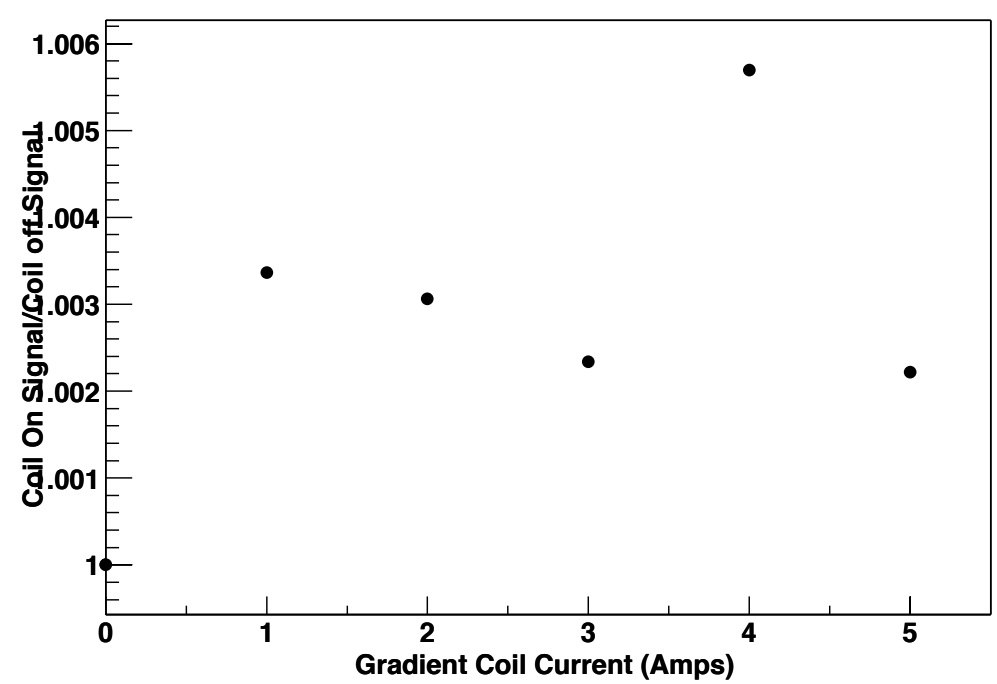

Figure 6.38: The effect on NMR signal height of the transverse gradient coil. 


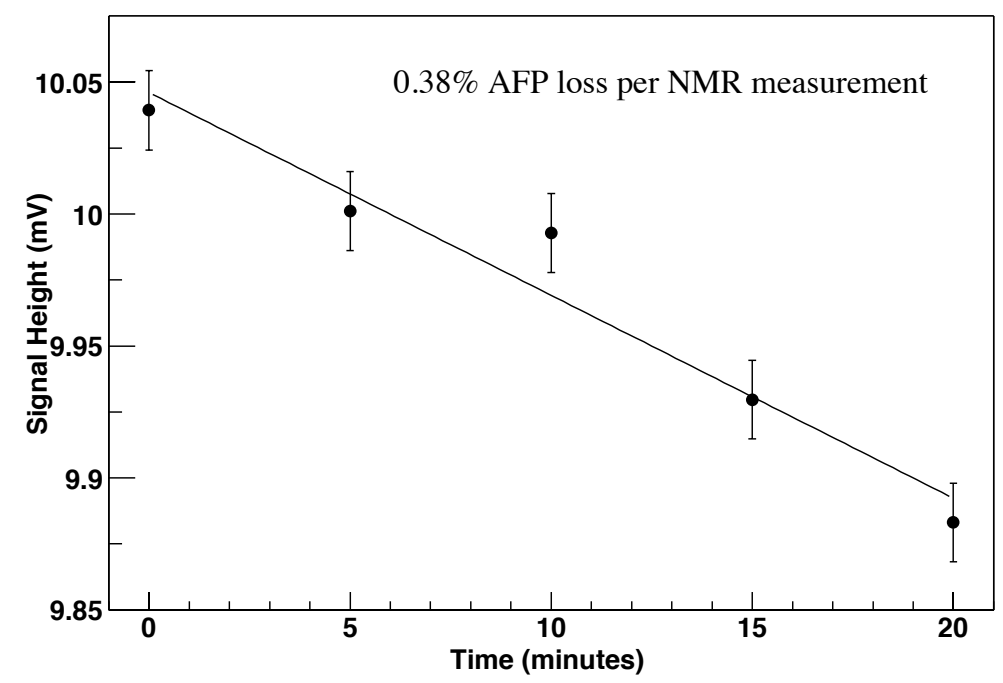

Figure 6.39: A plot of the effects of polarization loss due to AFP during NMR.

source of polarization loss occurs during the data taking. The polarization loss for a cell with no gradient coils on is shown in Fig. 6.39. The polarization loss for Shapiro was $\approx 0.5 \%$. The polarization loss for Virginia One was about $\approx 1.0 \%$ due to the increase in AFP loss due to the gradient coils. The measurement of these losses can vary by $30 \%$ depending on the amount of polarization.

\subsubsection{Analysis of the NMR Signals from Water Cells}

As stated previously, the polarization of the ${ }^{3}$ He cells are calibrated with the NMR signal from water. Unfortunately, the water signals are quite small (10000 times smaller than a typical helium signal) and are taken in a noisy environment. However, since the polarization doesn't change from sweep to sweep, the measurements can be taken thousands of times and averaged. Another complication of the water signal is that the relaxation times of water, $T_{1}$ and $T_{2}$, are on the order of the sweep time for an NMR measurement. This results in a slightly more complicated signal shape than the signals from the polarized ${ }^{3} \mathrm{He}$ cells.

In E97-103 there were 4 water calibrations done with a $40 \mathrm{~cm}$ water cell. The 

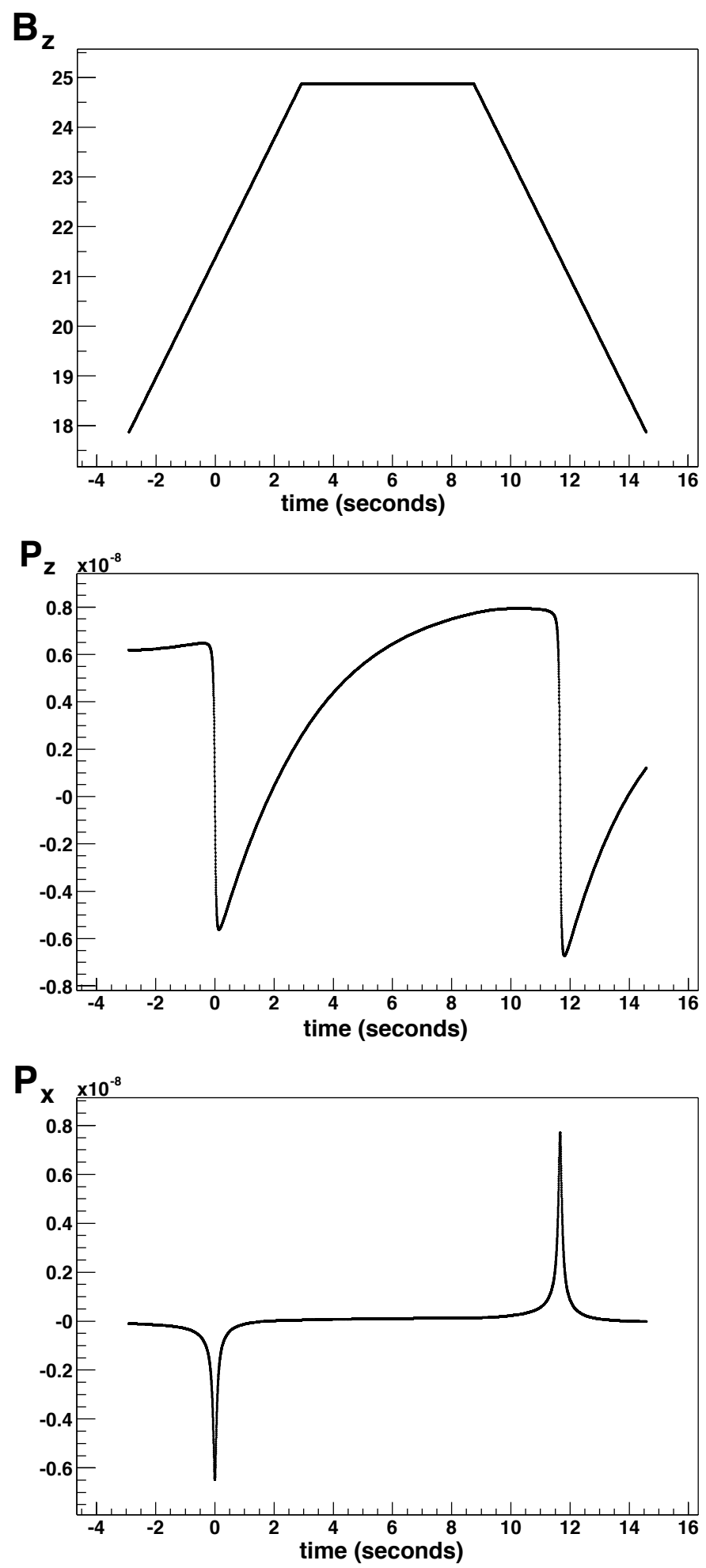

Figure 6.40: The magnetic field and $z$ and $x$ of a water cell undergoing AFP. 
number of sweeps used and signal height of these calibrations are shown in Table 6.14. The water calibration is swept from 18 Gauss to 25 Gauss rather than the 25 Gauss to 32 Gauss used for the polarized ${ }^{3} \mathrm{He}$ signal. This is to be able to use the same frequency for the RF coils, since the gyro-magnetic ratios are different for ${ }^{3} \mathrm{He}$ and water. There is also a $5.833 \mathrm{~s}$ hold when the field reaches $25 \mathrm{G}$ to wait for the water sample to relax. Because the water polarization is proportional to the holding field, the resonance when the field is being swept down will be larger than the field is being swept up because the starting field is larger.

As with the polarized ${ }^{3} \mathrm{He}$ NMR, the data files created by the LabView software that runs the water calibrations separates the water into four files: X Up, Y Up, X Down and Y Down. The "Up" and "Down" refer to whether the field was being increased or decreased through resonance. The "X" and "Y" refer to the $\mathrm{X}$ channel and $\mathrm{Y}$ channel in the lock-in amplifier. The $\mathrm{X}$ channel is generally locked on the signal, but sometimes the phase isn't set correctly so there is some signal in the Y channel. These can be easily combined by averaging and fitting each channel separately then combining the signal heights with $S=\sqrt{X^{2}+Y^{2}}$.

Because so many sweeps are averaged together, one has to worry about magnetic field drift in the signals. Since the magnetic field is not recorded in during the water calibrations and the voltage of the power supply drifts a little bit, the resonance of the water signal will not appear at the same $H$ for every sweep. This can be corrected by recording the currents and using the current-magnetic field calibrations shown earlier to correct each sweep.

Once the sweeps are field corrected, a calculation is made to determine the amount of noise in each sweep. This is done by fitting a line to each sweep and measuring the standard deviation of the data points around this line. The value produced by this process can then be put into a histogram as shown in Fig. 6.41. Exceptionally noisy sweeps can then be cut by cutting all the sweeps above a certain 


\begin{tabular}{lcccc}
\hline \hline Date & Num. Sweeps & Up Sig. $(\mu$ V) & Down Sig. $(\mu$ V) & Flux $\left(\mathrm{cm}^{2}\right)$ \\
\hline 1 Aug & 350 & $9.24 \pm 0.35$ & $11.11 \pm 0.30$ & 46.69 \\
31 Aug & 966 & $9.38 \pm 0.28$ & $9.78 \pm 0.53$ & 46.73 \\
1 Sep & 600 & $8.76 \pm 0.41$ & $11.19 \pm 0.45$ & 46.73 \\
17 Sep & 600 & $9.53 \pm 0.30$ & $10.01 \pm 0.56$ & 46.97 \\
\hline \hline
\end{tabular}

Table 6.14: The date, signal heights, the number of sweeps and the flux for each water calibration.

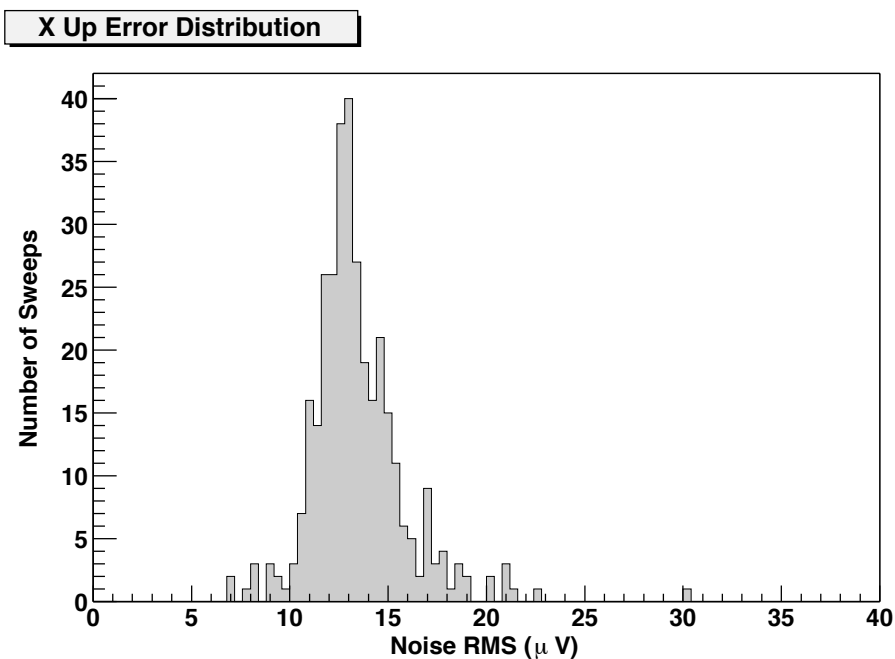

Figure 6.41: Average of the 4 water calibrations done in E97-103.

RMS level. In the case of this water analysis, no cuts were made for particularly noisy sweeps.

The sweeps are then averaged for all four files. The shape of the signal can be derived from Eq. 6.53. This set of equations can be approximated by [71]:

$$
P_{\mathrm{eff}}=k \sqrt{P_{x}^{2}+P_{y}^{2}+P_{z}^{2}}
$$

where $k= \pm 1$ and $P_{\text {eff }}$ is the polarization in the direction of the effective field. This can only be done if $T_{1}=T_{2}$ which is mostly the case $\left(T_{1}=3.0 \mathrm{~s}, T_{2}=2.7 \mathrm{~s}[71]\right)$. With this approximation the solution to the differential equations in Eq. 6.53 can be written:

$$
P_{\text {eff }}=e^{-\left(t-t_{i}\right) / T_{1}}\left[P_{e q}\left(t_{i}\right)+\frac{1}{T_{1}} \int_{t_{i}}^{t} e^{\left(u-t_{i}\right) / T_{1}} P_{e q}(u) d u\right]
$$




\section{Up Sweep}

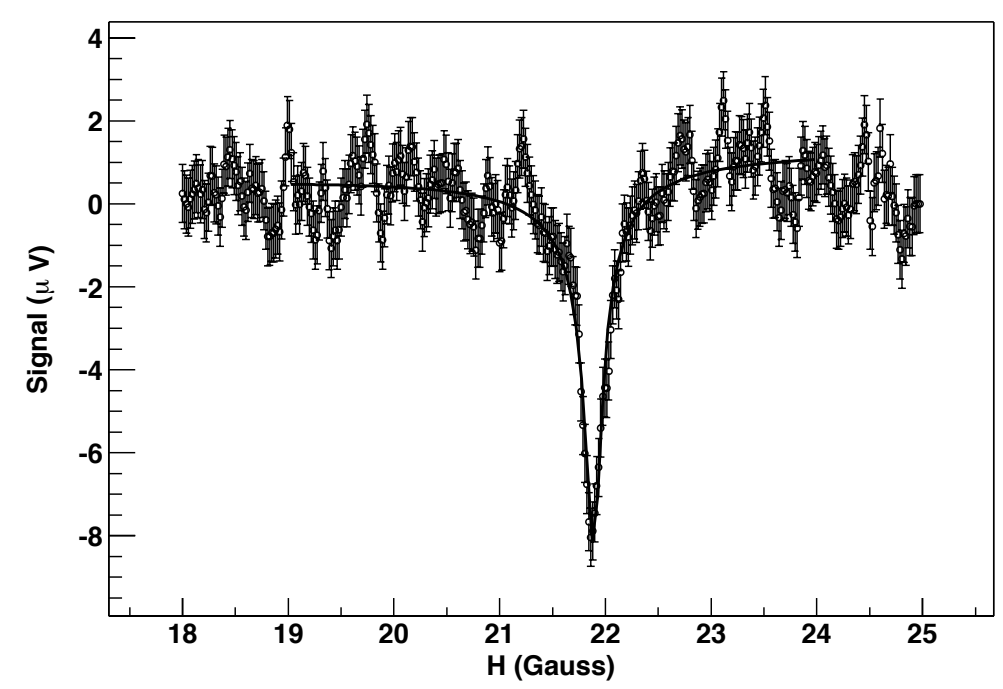

Figure 6.42: A fit of the Up sweep in the X channel of the 1 Aug 2001 water calibration.

where $t_{i}$ is the starting time of the sweep and

$$
P_{e q}=\chi \frac{H_{1}^{2}+\alpha t\left(H_{0}+\alpha t\right)}{\left.\sqrt{(} H_{1}^{2}+\alpha^{2} t^{2}\right)}
$$

where $\alpha$ is the sweep rate. The term $e^{\left(u-t_{i}\right) / T_{1}}$ in the integral can be expanded and only the first few terms kept, so that the final form of $P_{\text {eff }}(t)$ is analytical. This function is used to fit the water signals as shown in Fig. 6.42.

After fitting the functions, the signals are then the quantity $c_{w}^{\prime}$ can be plotted, where $c_{w}^{\prime}$ is defined as:

$$
c_{w}^{\prime}=\left(\frac{P_{w}}{h_{w}}\right)\left(\frac{\mu_{p} n_{p} \Phi_{t o t} G_{p}^{w} C_{\nabla}^{w} C_{\tau}^{w}}{\mu_{3} H e}\right) .
$$

The average of $c_{w}^{\prime}$ is plotted in Fig. 6.43. The values used for the parameters in Eq. 6.77 are listed in Table 6.15 .

\subsubsection{EPR polarimetry}

The second polarimetry system on the polarized ${ }^{3}$ He target measures the shift of the electron paramagnetic resonance (EPR) in the rubidium electron states when 


\begin{tabular}{cccc}
\hline \hline Parameter & Value & Uncertainty & Source \\
\hline$P_{w} C_{\nabla} C_{\tau}$ & $5.510 \mathrm{e}-9(\mathrm{Up}), 6.611 \mathrm{e}-9$ (Down) & $1.7 \%$ & Model of Bloch Eq. \\
$\mu_{p} / \mu^{3} \mathrm{He}$ & 1.313 & neg. & {$[57]$} \\
$G_{p}^{w}$ & 200 & $0.5 \%$ & Gain Measurements \\
$\Phi_{\text {tot }}$ & $46.69-46.97 \mathrm{~cm}^{2}$ & $2.3 \%$ (stat.) $\pm 1.0 \%$ (sys.) & Table 6.14 \\
$n_{p}$ & 2482 Amagats & $0.1 \%$ & {$[57]$} \\
\hline \hline
\end{tabular}

Table 6.15: List of Parameters used to calculate $c_{w}^{\prime}$

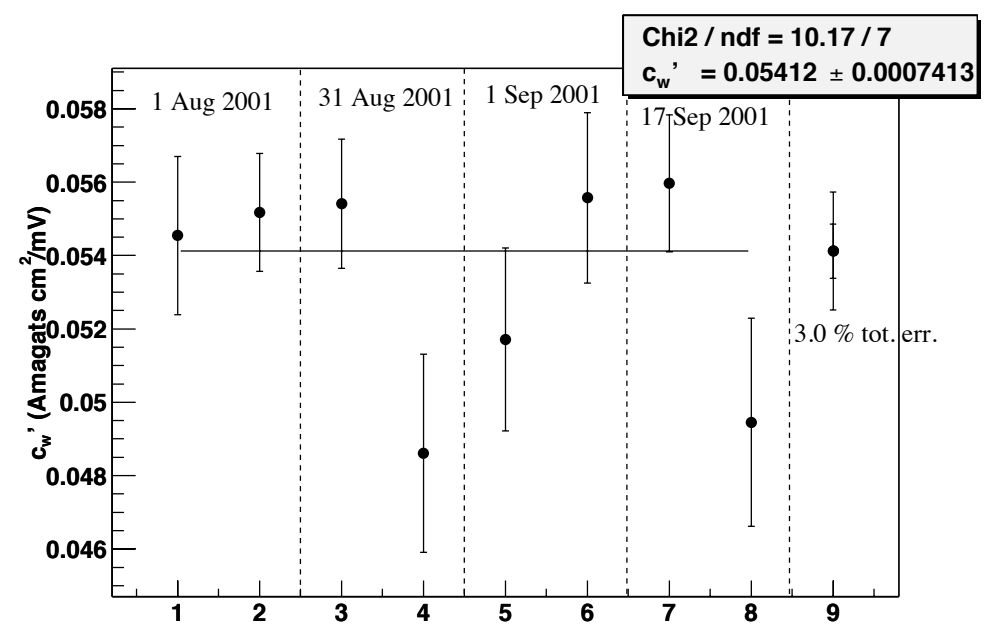

Figure 6.43: Average of the 4 water calibrations done in E97-103. 
the polarization direction of the polarized ${ }^{3}$ He nuclei is reversed. Specifically, what is being measured is the width of the Zeeman splitting between electron state $F=$ 3, $m=-3$ to $F=3, m=-2$ in Fig. 6.4. The width of this splitting depends on the total magnetic field. The total magnetic field is the sum of the holding field plus a much smaller contribution from the polarized ${ }^{3} \mathrm{He}$ nuclei. Changing the direction of polarization of the nuclei will also change the width of the Zeeman splitting.

The width of Zeeman splitting is measured by measuring the frequency at which electrons in the $F=3, m=-3$ state can absorb photons and be transfered to the $F=3, m=-2$ state. This is done with a excitation coil near the pumping chamber of the target cell. The frequency of excitation is quite high $\approx 11.6 \mathrm{MHz}$. However, the transition is quite narrow so it can be measured precisely.

Of course, one needs to know when the electron is making this electron state transition. The technique for doing this takes advantage of the method of optical polarization of rubidium. When the electron makes the transition from $F=3, m=$ -3 to $F=3, m=-2$, the electron can then absorb circularly polarized laser light and be excited to the ${ }^{5} P$ state. Electrons in the $F=3, m=-3$ cannot be excited by circularly polarized light. Therefore, when electrons are successfully making this transition there will be an immediate increase in outgoing photons from electrons dropping from the ${ }^{5} P$ to ${ }^{5} S$ state, as these excited electrons decay. Because, of collisional mixing, which causes the electron state transition due to rubidium-rubidium collisions, there will be increased amount of photons at a range of frequencies. The two photon frequencies that indicate the resonance most strongly are the D1 $(795 \mathrm{~nm})$ and the D2 $(785 \mathrm{~nm})$ transitions. For EPR polarimetry, the D2 resonance is the one detected since the D1 resonance is lost in the huge intensity of laser light at $795 \mathrm{~nm}$.

The setup for EPR polarimetry is shown in Fig. 6.44. The normal procedure for performing a measurement is to set the excitation function generator (Wavetek 


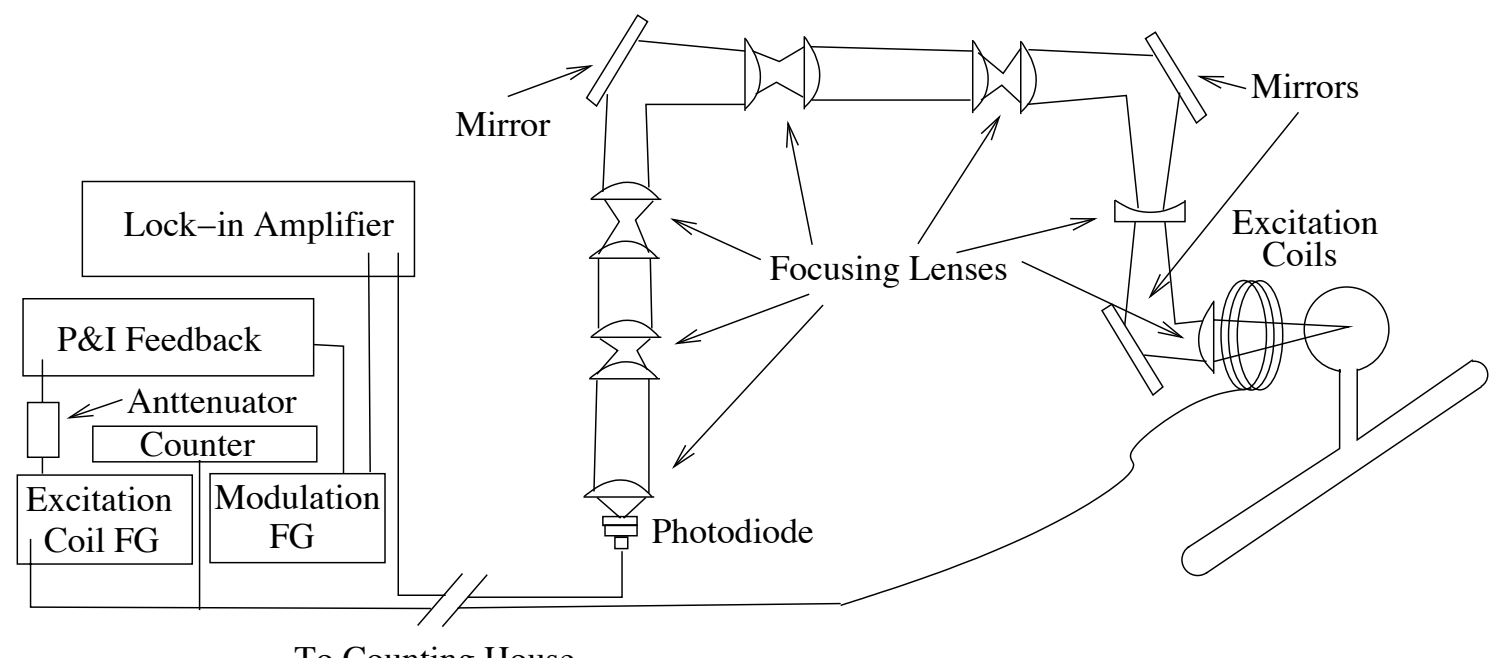

To Counting House

Figure 6.44: The equipment setup for EPR polarimetry.

80) to the frequency where the EPR transition is expected. This will power the excitation loops near the pumping chamber. The frequency to the excitation loops is modulated by another function generator (SRS DS345) at $200 \mathrm{~Hz}$. This helps to separate the signal being caused by the excitation coils from background. The frequency from the excitation coil function generator is monitored precisely by an electronic counter (SRS SR620).

Light from the pumping chamber of the cell is focused through a series of lenses to a photodiode which produces a signal which is proportional to the intensity of light that it absorbs. In principle, the lenses are unnecessary and the photodiode can be place directly next to the pumping chamber; however, the target area is a high-radiation environment when the electron beam is on and radiation damages the photodiodes. Therefore, a series of lenses is necessary to keep the photodiode working.

The photodiode signal is directed to a lock-in amplifier which is locked into a frequency of $200 \mathrm{~Hz}$. When the excitation loop is not at the right frequency for the EPR transition to occur there is no signal in the lock-in amplifier. When the frequency is found there will be a small signal. However, when the polarization of 


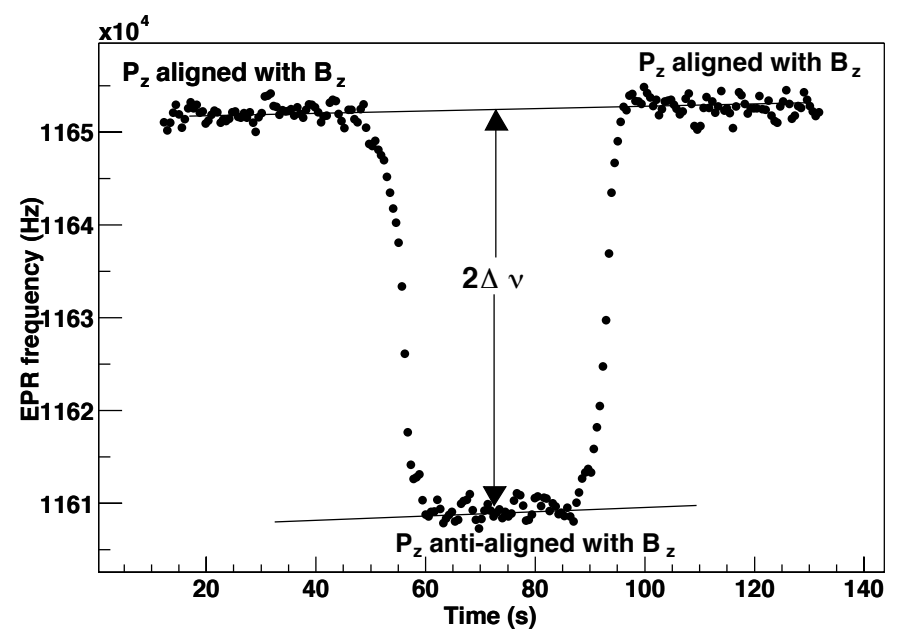

Figure 6.45: The EPR resonance plotted vs. time during AFP.

the ${ }^{3}$ He nuclei is reversed the frequency of this signal will move rapidly. Therefore, a set of electronics is used to lock-into the EPR signal and adjust the frequency of the excitation coil function generator so that it is always exciting the EPR transition. This set of electronics is known as the P\&I feedback box. Further details about the electronics set-up can be found in [41] and [57].

Once the signal is locked on by the P\&I feedback system then the polarization of the ${ }^{3} \mathrm{He}$ is reversed using AFP. Unlike NMR where the holding field was ramped through resonance, the holding field is kept the same and the RF coil frequency is ramped through resonance. This is because the resonance is very sensitive to the magnitude of the holding field, but not sensitive at all the RF field. Once the polarization is reversed, the new EPR frequency is recorded for a period of 20-30s. Then the polarization is flipped back to the original orientation. An example of this process is shown in Fig. 6.45.

The frequency difference $(2 \Delta \nu)$ is proportional to the polarization as shown here:

$$
2 \Delta \nu=\frac{4 \mu_{0}}{3} \frac{d \nu_{\mathrm{EPR}}}{d B} \kappa \mu_{3} \mathrm{He}_{\mathrm{pc}} P_{3 \mathrm{He}}
$$

where $\mu_{3} \mathrm{He}$ is the magnetic moment of ${ }^{3} \mathrm{He}, n_{\mathrm{pc}}$ is the number density of the pumping 


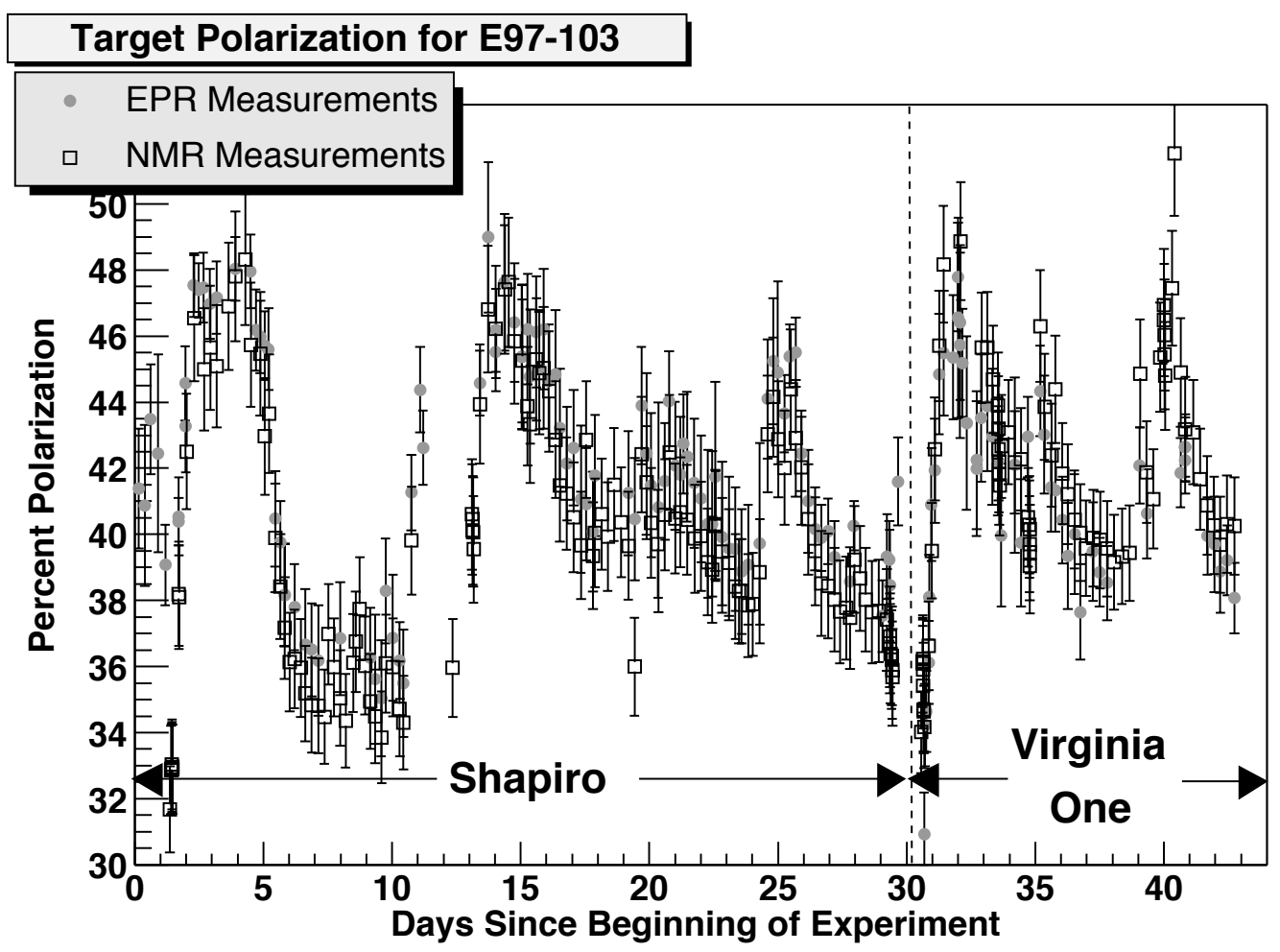

Figure 6.46: The NMR calibration constant from EPR measurements, $c_{\mathrm{EPR}}^{\prime}$.

chamber, $P_{3_{\mathrm{He}}}$ is the polarization of the ${ }^{3} \mathrm{He}$ in the pumping chamber and the derivative $d \nu_{\mathrm{EPR}} / d B$ and the $\kappa$ are constants from atomic physics experiments. Detailed analysis of $d \nu_{\mathrm{EPR}} / d B$ and $\kappa$ can be found in [57] and [41].

\subsubsection{Combining Data from EPR and NMR}

For E97-103, every time a polarimetry measurement was needed, usually every 4-6 hours, both an NMR and EPR measurement was done. The results from these calibrations are shown in Fig. 6.46.

The method of combining these two set of data was to use the EPR measurements as a constant calibration of the NMR measurements. This was done because NMR signal can be measured much more precisely ( $0.005 \%$ for each measurement) than the EPR signal (1-4\% for each measurement). However, the EPR measurements give an absolute value for the polarization whereas the NMR measurements 


\begin{tabular}{|c|c|c|}
\hline Cell & Constant (Amagats $\mathrm{cm}^{2} / \mathrm{mV}$ ) & 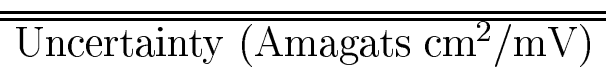 \\
\hline Shapiro & 0.05220 & 0.00155 \\
\hline Virginia One & 0.05602 & 0.00168 \\
\hline Average & 0.05400 & 0.00157 \\
\hline
\end{tabular}

Table 6.16: EPR Calibration Constants for NMR.

\begin{tabular}{ccc}
\hline \hline Parameter & Error & Note \\
\hline$S_{\mathrm{NMR}} / P_{\mathrm{EPR}}$ & $0.6 \%$ & Statistical Error \\
$S_{\mathrm{NMR}} / P_{\mathrm{EPR}}$ & $1.5 \%$ & Systematic Error \\
$n$ & $1.0 \%$ & Systematic Error \\
$\Phi$ & $2.4 \%$ & Systematic Error \\
\hline \hline
\end{tabular}

Table 6.17: List of errors associated with NMR calibration from EPR.

need to be calibrated. There is value for the calibration from the water signal, but it can be improved by including information from the EPR signal.

To extract an NMR calibration from EPR signal one can calculate the EPR calibration constant $c_{\mathrm{EPR}}^{\prime}$ for each pair of NMR-EPR measurements:

$$
c_{\mathrm{EPR}}^{\prime}=\frac{S_{\mathrm{NMR}}}{P_{\mathrm{EPR}}\left(n_{\mathrm{pc}} \Phi_{\mathrm{pc}}+n_{\mathrm{tc}} \Phi_{\mathrm{tc}}+n_{\mathrm{tt}} \Phi_{\mathrm{tt}}\right) C_{\nabla} C_{\tau}}
$$

where $S_{\mathrm{NMR}}$ is the NMR signal from polarized ${ }^{3} \mathrm{He}, P_{\mathrm{EPR}}$ is the polarization extracted from the EPR measurement, $n_{\mathrm{pc}}, n_{\mathrm{tc}}$ and $n_{\mathrm{tt}}$ are the densities of the various chambers, $\Phi_{\mathrm{pc}}, \Phi_{\mathrm{tc}}$ and $\Phi_{\mathrm{tt}}$ are the fluxes from the various parts of the cell, and $C_{\nabla}$ and $C_{\tau}$ are the NMR signal correction factors from the gradient and lock-in time constant. $c_{E P R}^{\prime}$ is a constant for all NMR measurements and can be compared directly to $c_{w}^{\prime}$ from the water calibration. The average values of $c_{E P R}^{\prime}$ for E97-103 is presented in Table 6.16. The errors associated with $c_{\mathrm{EPR}}^{\prime}$ are presented in Table 6.17 .

The the two calibration constants and their weighted average is shown in Table 6.18. A list of systematic errors that go into every polarization value is shown in Table 6.19. The polarizations using this method are assigned to each run using a linear interpolation. 


\begin{tabular}{|l|c|c|}
\hline \hline Calibration Method & Constant & Uncertainty \\
\hline Water NMR & 0.05412 & 0.00161 \\
EPR & 0.05400 & 0.00157 \\
\hline Average & 0.05406 & 0.00112 \\
\hline \hline
\end{tabular}

Table 6.18: Calibration Constants for NMR signals. Values in Amagats $\mathrm{cm}^{2} / \mathrm{mV}$

\begin{tabular}{|l|c|c|}
\hline \hline Description & Parameter & Uncertainty \\
\hline Calibration Constant & $c^{\prime}$ & $2.1 \%$ \\
NMR Signal Height & $S_{N M R}$ & $0.5 \%$ \\
Flux (all chambers) & $\Phi$ & $2.4 \%$ \\
Density & $n$ & $1.0 \%$ \\
Corrections & $C_{\nabla} C_{\tau} G_{p}$ & $1.0 \%$ \\
\hline Total & $P_{N M R}$ & $3.7 \%$ \\
\hline \hline
\end{tabular}

Table 6.19: List of systematic errors in the polarization measurements in E97-103.

\section{$\underline{6.10 \quad \text { Masing }}$}

Masing is a phenomenon associated with the polarized ${ }^{3} \mathrm{He}$ target that has two characteristics : a sudden loss of polarization during AFP and a polarization threshold where masing is not seen [57]. The results of masing can be seen in Fig. 6.47. In this figure, the first value of $2 \Delta \nu$ from the first magnetization reversal shows a high polarization, but when the magnetization is in the reversed state it begins to lose polarization until the magnetization is flipped again. This behavoir continues until the system reaches a certain polarization at which this masing behavior stops.

The source of this phenomena has been linked to the resonance frequency of the pick-up coils [57]. In the case of E97-103, the resonance frequency is $180 \mathrm{kHz}$ where the RF coil frequency is $91 \mathrm{kHz}$. The pick-up coil placement and configuration was the same for both cells. However, Virginia One showed strong masing at high polarization, whereas Shapiro showed none. It is unknown why one cell would show this phenomena and not the other. It has been speculated that the rubidium inside the pumping chamber plays a part in this phenomena. The amount of rubidium in Shapiro and Virginia One were about the same. 


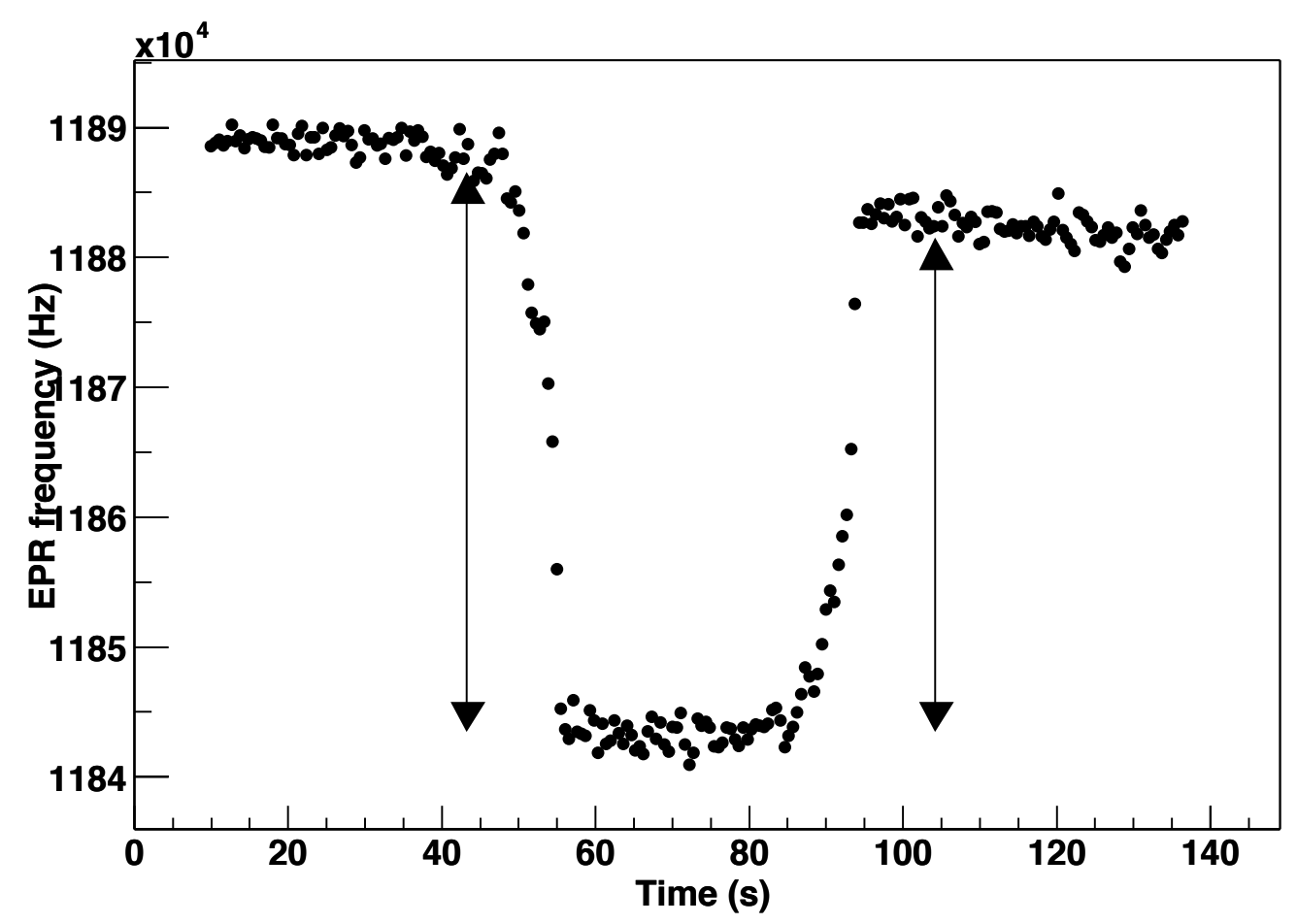

Figure 6.47: An example of masing occurring during an EPR measurement.

In the case of Virginia One, the gradient coils were turned on for the duration of its use. The higher gradients suppress the masing effect and allow the cell to get to high polarizations, at the cost of higher AFP losses. Masing has been a continuing problem with this system and requires further study.

\subsection{Reference Cell}

In addition to the polarized ${ }^{3} \mathrm{He}$ cells, the Jefferson Lab target also provides for electron scattering from a carbon foil target and a reference cell target that can be filled with nitrogen, ${ }^{4} \mathrm{He}$ or ${ }^{3} \mathrm{He}$. These targets, plus the polarized ${ }^{3} \mathrm{He}$ target are included on the same target ladder, which can be moved in and out of the beam by a vertical lifter.

The carbon foil target has a series of five thin carbon graphite foils that are used for optics studies and measurements of false asymmetries. The reference cell is 
used to measure yield ratio of nitrogen to ${ }^{3} \mathrm{He}$ for the dilution factor. The reference cell is connected to series of pumps and valves that allow gas to be pumped in and out the cell remotely. Unfortunately, the reference cell system leaked throughout the experiment and approximations had to be made for the actual pressure in the cell. 


\section{CHAPTER 7}

\section{The Hall A Spectrometers and Detector Package}

\subsection{Spectrometer Magnets}

Hall $\mathrm{A}$ is equipped with two high-resolution spectrometers (known as the left and right spectrometers) which are nearly identical in design. The spectrometers transport charged particles covering a narrow range in scattering angle and momentum into a detector package. The use of these spectrometers minimizes background and allows precise cross-section measurements. The spectrometers can be positioned around the target at angles from $12.5^{\circ}$ to $130^{\circ}$ (the left spectrometer can go to $150^{\circ}$ ). For E97-103, the spectrometers were set at symmetrical angles and used as independent single-arm detectors to double the rate of data taking of scattering electrons.

Each spectrometer has four magnets, as shown in Fig. 7.1. The first two magnets are superconducting quadrapole magnets that focus the scattered electrons before the entrance of the dipole magnet. The first quadrapole, Q1, focuses the electrons vertically at the focal plane and the second quadrapole, Q2, focuses the electrons horizontally at the focal plane [75]. The third magnet is a superconducting dipole which bends the electrons $45^{\circ}$ vertically. The current setting in the dipole magnet determines the momentum of the electrons which make it into the detector package. The fourth magnet is another superconducting quadrapole magnet which does additional horizontal focusing at the focal plane. The characteristics of the spectrometer are given in Table 7.1 [75].

The spectrometer also has a tungsten collimator before the first quadrapole 


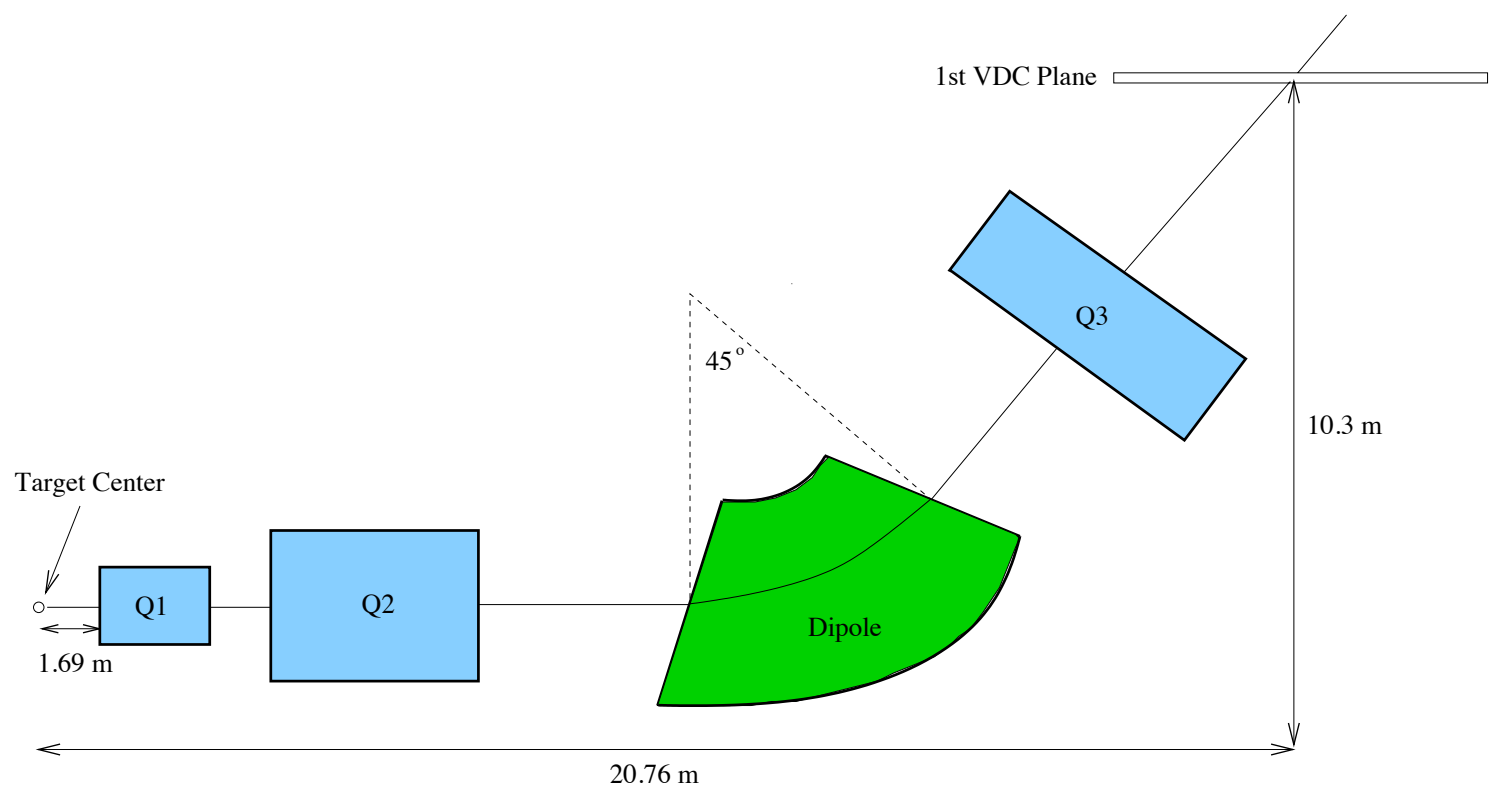

Figure 7.1: A diagram of the Hall A magnets of the high resolution spectrometers.

\begin{tabular}{lc}
\hline \hline Characteristic & value \\
\hline Momentum Range & $0.3-4.0 \mathrm{GeV}$ \\
Target to Detector Length & $23.4 \mathrm{~m}$ \\
Momentum Acceptance & $\pm 4.5 \%$ \\
Horizontal angular acceptance & $\pm 28 \mathrm{mr}$ \\
Vertical angular acceptance & $\pm 60 \mathrm{mr}$ \\
Solid angle & $6 \mathrm{msr}$ \\
\hline
\end{tabular}

Table 7.1: Characteristics of the Hall A spectrometers. 


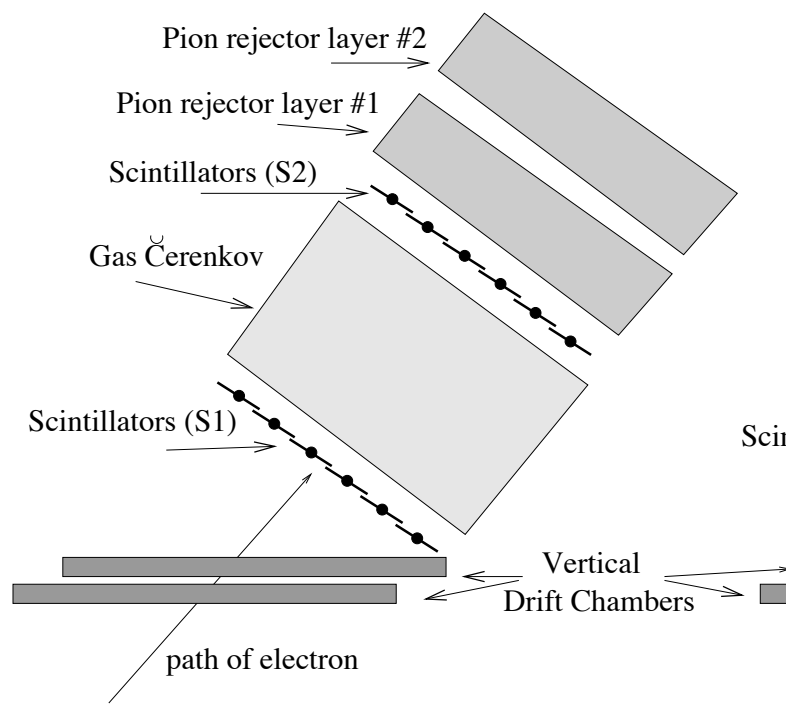

Left Arm Detector Package

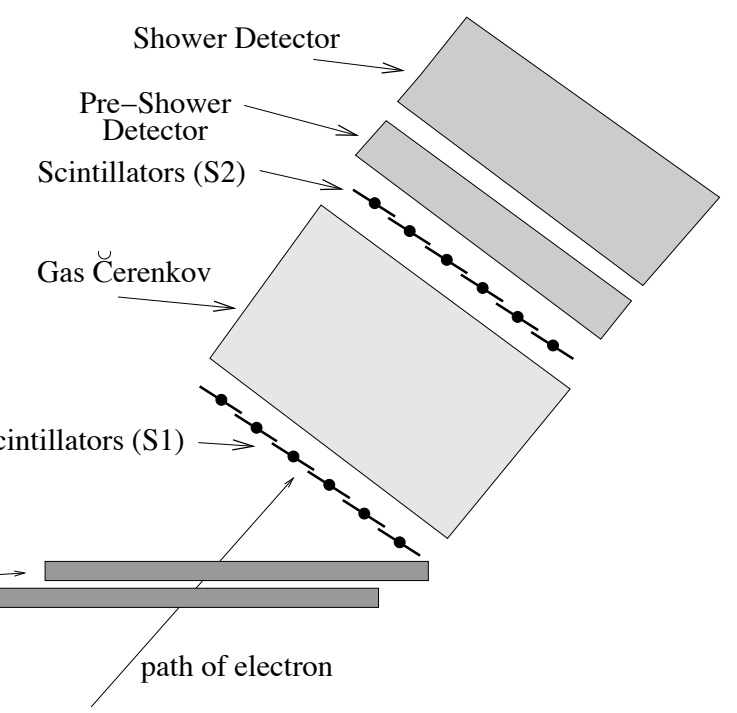

Right Arm Detector Package

Figure 7.2: A diagram of the detector package used in the Hall A spectrometers.

which limits the angular acceptance of the spectrometer. It is machined and positioned to have a $6 \mathrm{msr}$ acceptance. It was used for most of the elastic runs.

\subsection{Detector Package}

\subsubsection{Overview}

Once the scattered electrons exit the spectrometer magnets, they enter a set of devices known as the detector package. In E97-103, the detector package's purpose was to identify scattered electrons and to characterize their momentum and direction. This was done with four type of devices : vertical drift chambers (VDCs), scintillators trigger planes, a gas Cerenkov and lead-glass shower detectors. These devices are shown in Fig. 7.2. The detector packages in the left and right spectrometers are almost identical. The major difference between the two is the configuration of the lead-glass shower detectors. The left arm has two layers of lead-glass detectors of equal thickness and the right arm also has two layers, but with one layer thicker than the other. 


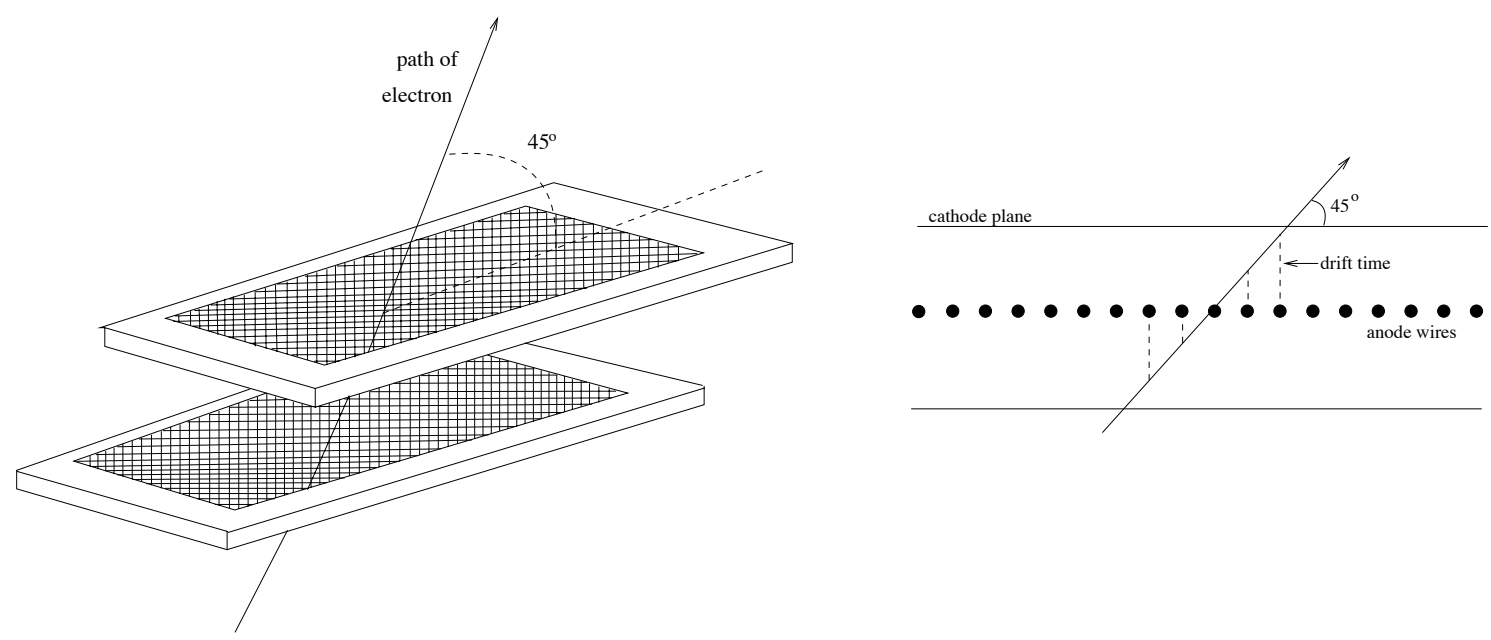

Figure 7.3: Two diagrams of the vertical drift chambers. The left diagram shows the path of an electron through the two VDCs. The right diagram shows an electron path firing five wires.

\subsubsection{Vertical Drift Chambers}

The purpose of the vertical drift chambers (VDCs) is to determine the position and direction of charged particles that pass through them. VDCs can measure position by using planes of wires where each wire will create a signal if a charged particle passes near it. By using multiple planes of these wires, the direction of the particle can be reconstructed.

There are two identical VDCs in each spectrometer placed at $45^{\circ}$ to the central electron path [44]. Each VDC is a closed chamber filled with gas (62\% argon and $38 \%$ ethane gas) and two parallel planes of 400 gold-plated wires. Each plane of wires is enclosed in a layer of aluminized mylar that is kept at high negative voltage, while the wire planes are grounded. The wires in the two planes run perpendicular to each other in the plane of the VDCs, as shown in Fig. 7.3.

Charged particles that pass through the VDCs ionize the gas in the chamber. The newly formed ions will head towards the negatively charged mylar. The electrons freed in the process move towards the grounded wires. If a charged particle passes near a wire, the positive ions moving away from it will induce a detectable 
negative signal in the wire [76]. Because of the $45^{\circ}$ angle and the close spacing of the wires (4.243 mm spacing), a particle passing through a VDC plane will typically create a signal in 5 wires [75]. Because of occasional inefficiencies in the wire signals, only 3 wire signals are needed to be considered a good track in that plane.

The amount of time it takes for the effects of ionization to reach a wire from the particle path is known as drift time. The distance from the wire to the particle path can be accurately determined from the drift time. Drift time is measured by a Time to Digital Converter (TDC) [77] which is a electronic device that acts like a clock. The TDCs are started when the first signal from a wire occurs and the TDCs are stopped by an event at the scintillators. The position of the charged particle can be deduced from the difference in signal times from the TDCs from each wire of the VDCs.

The VDC efficiency can be measured by looking at the quality of the path reconstruction from its output. This is done by going through a set of events and counting the number the possible paths it could have had going through the VDCs. Ideally, each electron will have only one possible reconstructed path. However, if there is wire noise or an inefficient wire then there can be multiple paths the particle could have taken.

Fig. 7.4 shows the VDC efficiency for each kinematic. The conditions for this analysis were to look only at events that have a signal in either the shower and pre-shower detectors in the right arm or both pion-rejectors in the left arm. This made sure that most of the events were scattered particles and not noise or cosmic rays. For each kinematic, six sets of events (or runs as they are called) were chosen, three from the left arm and three from the right arm. There were no zero track events from events that had shower and pre-shower (or both pion rejector) signals. As expected, the efficiency of VDCs is correlated to the rates as the elastic kinematic had a significantly higher rate than the rest. The DIS kinematics all had tracking 

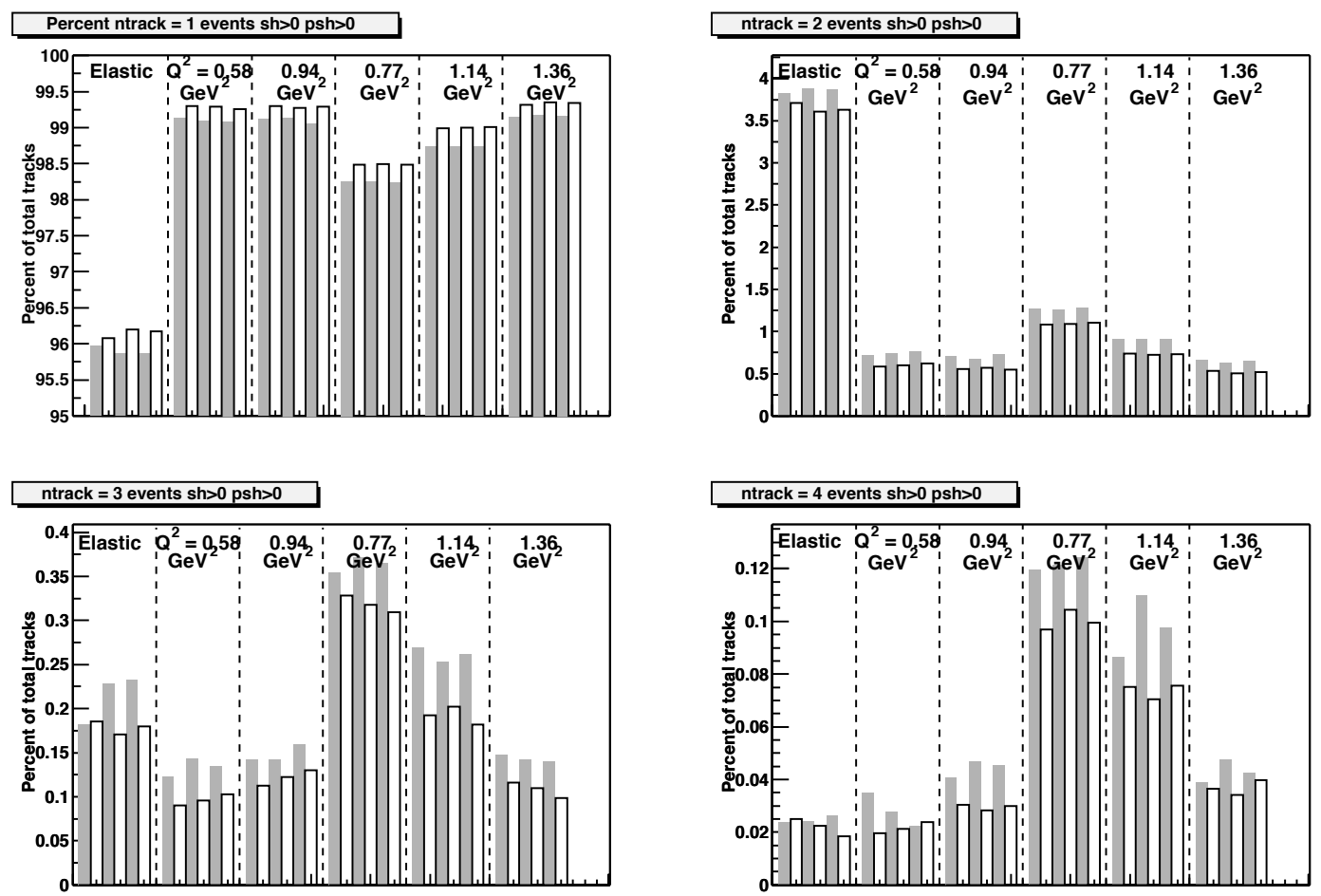

Figure 7.4: A plot of the percentage of events assigned to one, two, three and four track events by reconstructing the TDC information from the VDCs. Six runs are plotted for each kinematic, three for the left spectrometer arm and three for the right. The runs were chosen at random from the kinematics. 


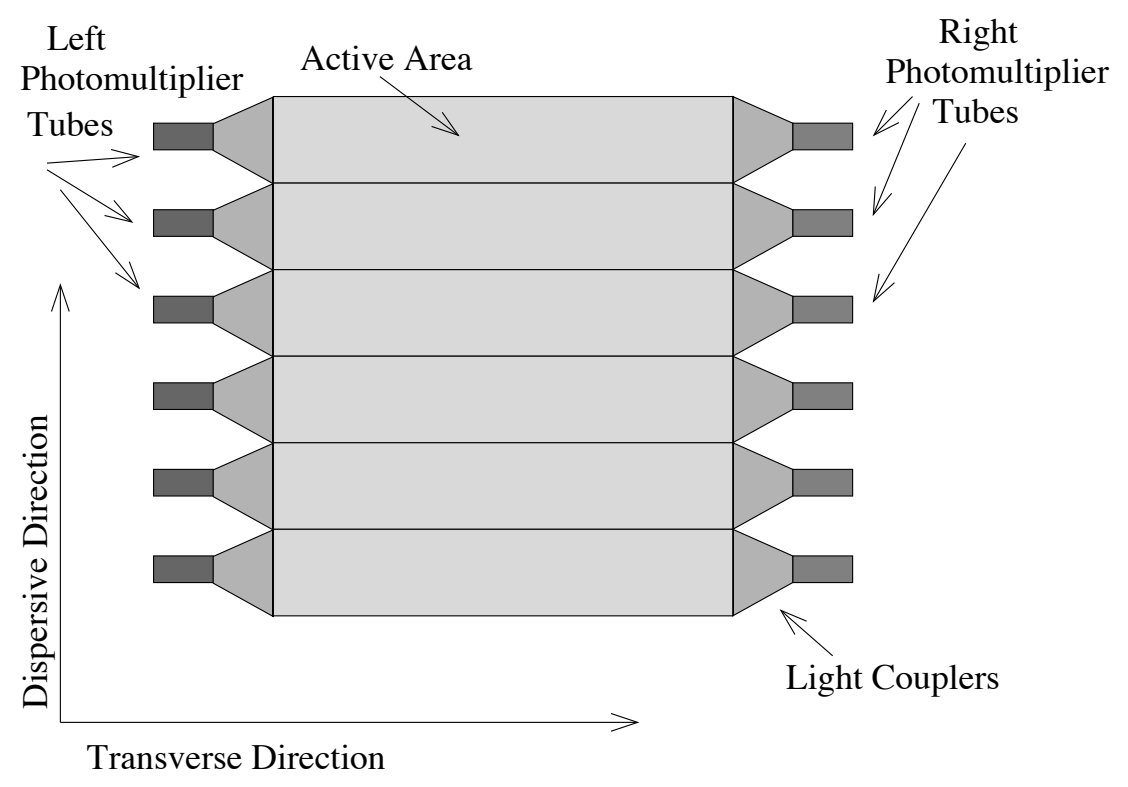

Figure 7.5: A diagram of the scintillator paddles.

efficiencies higher than $98 \%$.

\subsubsection{Scintillator and Trigger Electronics}

The scintillator planes S1 and S2 are used to trigger the data acquisition system and as additional method of tracking charged particles through the spectrometer. The scintillators, shown in Fig. 7.5, are made of Bicron BC-408 plastic and are 1.27 cm thick [75]. There are six scintillators paddles in each plane and each scintillator paddle has two photo-multiplier tubes at each end.

Charged particles generate light as they pass through a scintillator. The light travels through the scintillator to the photo-multipler tubes at the end of scintillator. The intensity and timing of the light is monitored by ADC (Analog to Digital Converter) and TDC electronics. Both photo-multiplier tubes have to register a signal from the event for an event to be considered good.

The scintillators are used to identify and classify events. The paddles in the S2 planes in both arms are used to trigger events. There are four types of events labeled T1,T2,T3 and T4. T1 and T3 events are from particles that cause signals in 

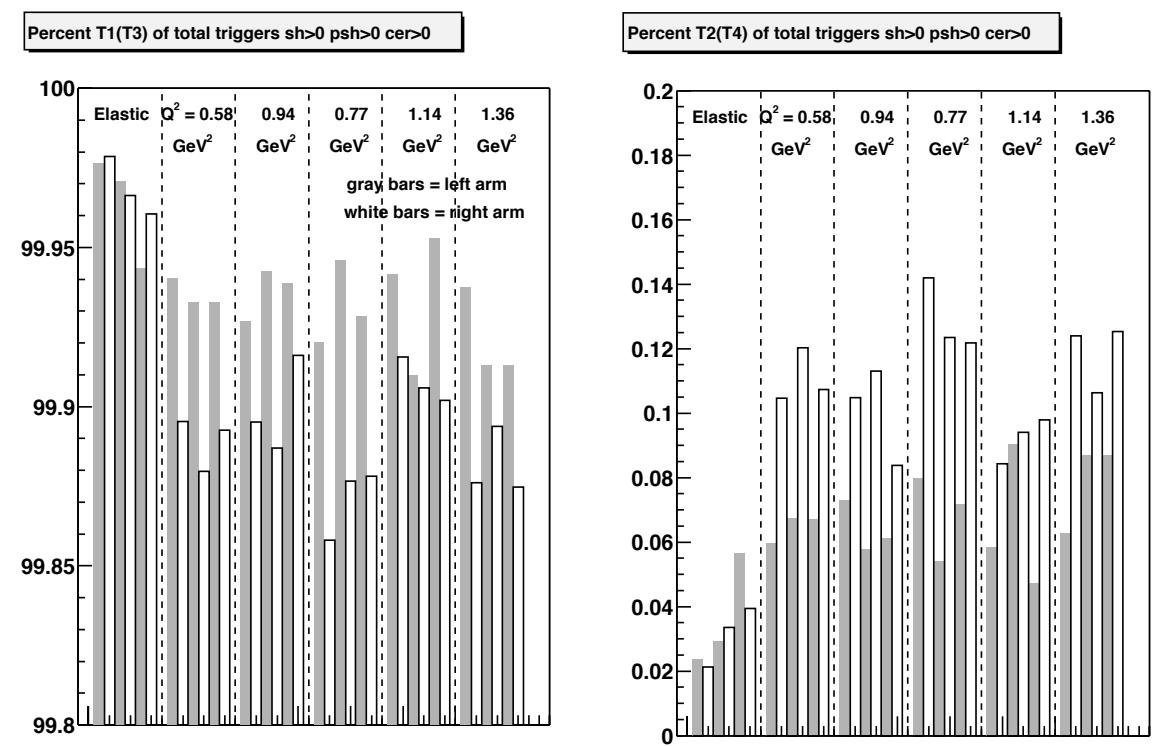

Figure 7.6: A plot of the scintillator efficiencies in the left and right spectrometers. Six runs are chosen for each kinematic, three from the left arm and three from the left arm. The runs are chosen at random.

a paddle in the S2 plane as well as the corresponding paddle in the S1 plane (or the paddle right above or below to account for the particle traveling at an angle to the scintillator plane). T1 events are for the scintillator planes in the right spectrometer arm and T3 are for the scintillator planes in the left spectrometer arm. T2 and T4 events are from two sets of particles. The first set are events that cause signals in a combination of S1 and S2 paddles that do not correspond to a T1 or T3 event. The second are for particles which cause a signal in a paddle in the S2 plane and have a Cerenkov signal, but did not cause a signal in any S1 paddle. T2 events are for the right spectrometer arm, while T4 events are for the left spectrometer arm.

Unless the rates are high, T2 and T4 events are considered bad events. This is because the events were caused by particles on the edge of the acceptance or from cosmic rays. The scintillator efficiency can be measured by:

$$
\eta=\frac{T_{1}}{T_{1}+T_{2}} \text { for the right HRS, }=\frac{T_{3}}{T_{3}+T_{4}} \text { for the left HRS }
$$

where $T_{n}$ is the number events of type $n$ in the sample. This has been done for E97- 
103 and is plotted in Fig. 7.6. The scintillator efficiency was measured by counting T1,T2,T3 and T4 events that had a signal in the C̆erenkov and lead glass detectors. Again, the Čerenkov and lead glass detectors are to make sure almost all the events are coming from scattering particles.

\subsubsection{Gas C̆erenkov Detector}

The gas C̆erenkov detector is used for separating electrons from other types of charge particles, namely pions. C̆erenkov light is created when a charged particle, traveling in a medium, is traveling faster than the speed of light in that medium. The threshold for this C̆erenkov light is determined by the index of refraction of the medium. If particles aren't traveling fast enough to exceed this threshold then no Cerenkov light is emitted.

The principle of the C̆erenkov detector is to separate particles that are traveling fast enough to create C̆erenkov light from slower particles. Electrons and pions coming out of the spectrometer will have the same momentum, but different velocities because of their mass differences. Electrons will make a measurable amount of $\breve{C}$ erenkov light, while the heavier pions won’t.

In the Hall A C̆erenkov detectors, shown in Fig. 7.7, the medium used is $\mathrm{CO}_{2}$ held at 1 atmosphere. The threshold for this type of detector is $17 \mathrm{MeV}$ for electrons and $4.87 \mathrm{GeV}$ for pions [75]. The C̆erenkov light created by the electrons is focused on a set of ten photo-multiplier tubes. The signals from these phototubes are sent to ADCs and summed. The sum of the ADC signals is the total light generated by the particle.

There is a significant background caused by electrons knocked out of material by pions before reaching the C̆erenkov detector. These electrons knocked out electrons are known as $\delta$-electrons [78], and can produce Č Cerenkov light and create ADC signals. Generally, $\delta$-electrons aren't traveling in the same direction as the scattered 


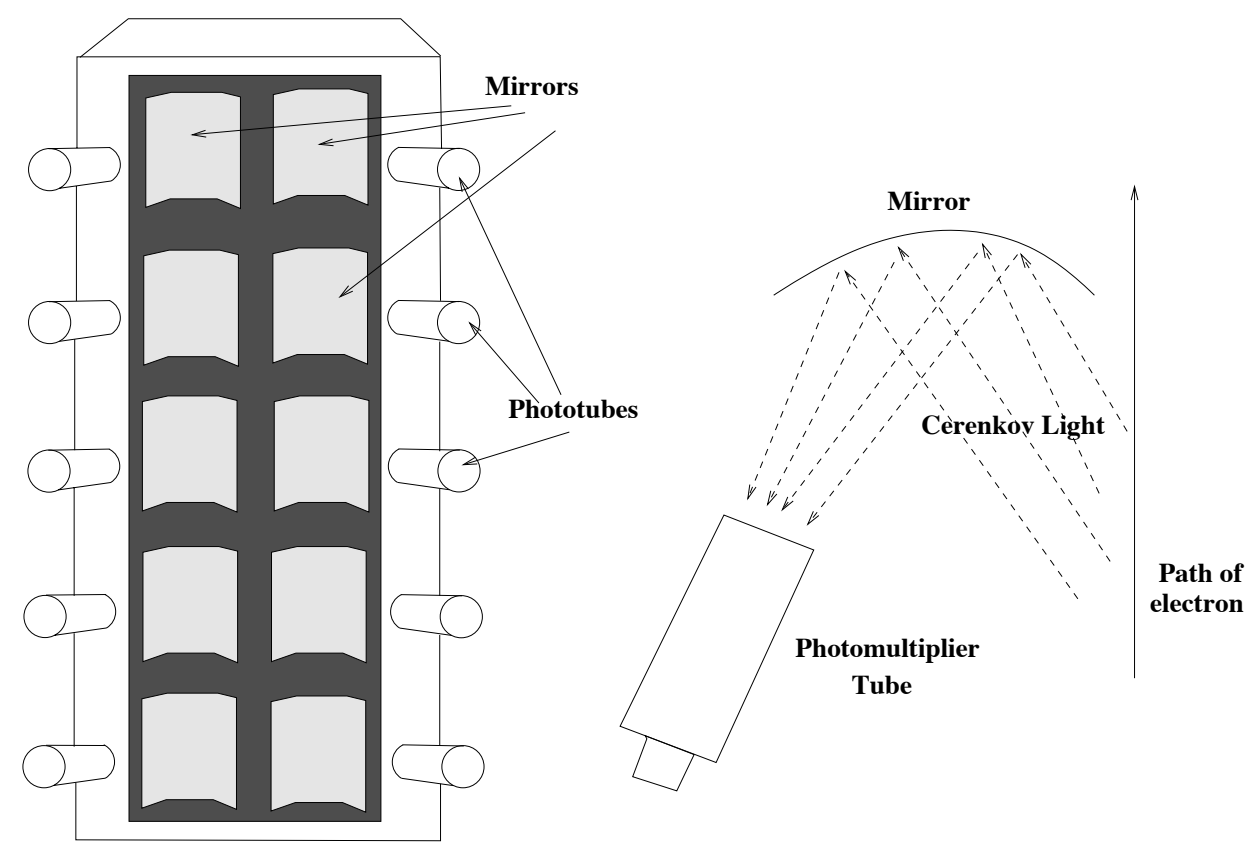

Figure 7.7: Two diagrams of the gas C̆ernkov detector. The left shows the front of the detector with the cover off. The right is a schematic depicting the mirror collecting Cerenkov light and reflecting it into a phototube.

electrons and, as a result, will create less Čerenkov light. Additional help identifying $\delta$-electrons comes from the lead-glass detectors.

\subsubsection{Lead-Glass Shower Detectors}

Lead-Glass detectors are another way of separating scattered electrons from other types of particles. High-energy charged particles will create bremsstrahlung radiation when traveling through the lead glass. The bremsstrahlung will in turn create $\mathrm{e}^{-} / \mathrm{e}^{+}$pairs which will also bremsstrahlung and create new particles [75]. This process is known as a shower and the energy of the particle is proportional to intensity of the photons emitted by the bremsstrahlung from the $-/ \mathrm{e}^{+}$pairs during the shower. Bremsstrahlung decreases with increased mass as $1 / m_{\text {particle }}^{2}$ Therefore, the intensity of the light created by pions and heavier particles will be significantly less than electrons.

A lead-glass detector consists of a rectangular chunk of lead-glass with a photo- 


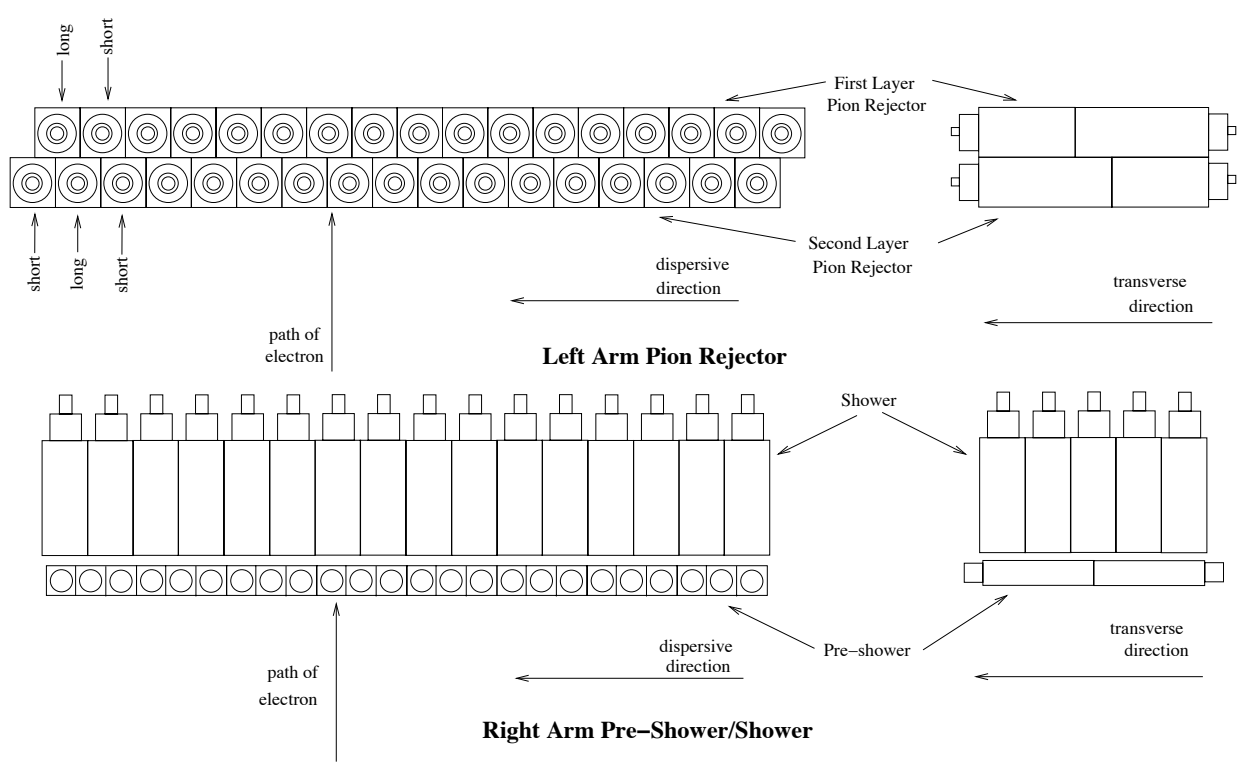

Figure 7.8: A diagram of the phototube and lead glass configurations in the pre-shower, shower and pion rejectors.

multiplier tube glued to the end. The lead-glass detectors are assembled differently in each spectrometer arm. In the left arm, there are 17 long block $(14.5 \times 14.5 \times 35$ $\mathrm{cm})$ detectors and 17 short block $(14.5 \times 14.5 \times 30 \mathrm{~cm})$ detectors assembled into two layers as shown in Fig. 7.8. The photo-multipliers are perpendicular to the path of the particles through the spectrometers. This assembly is known as the 'pion-rejector'. The lead-glass detectors in the right arm, are made of two types of lead-glass blocks. The smaller blocks that make up the 'pre-shower' are made of $4810 \times 10 \times 35 \mathrm{~cm}$ blocks assembled with the photo-multiplier tubes perpendicular to the path of the electron beam. The larger blocks are a $10014.5 \times 14.5 \times 35 \mathrm{~cm}$ blocks arranged into a $5 \times 20$ rectangle with the photo-multiplier tubes aligned with the particle path, as shown in Fig. 7.8.

The photo-multiplier tubes are connected to ADCs so that the intensity of light corresponding to each event is recorded. In E97-103, there were two distinct peaks corresponding to electrons and pions. The two layers help to separate the tail of the pion peak from the electron peak. At higher energies the separation between the 


\begin{tabular}{|c|c|c|c|}
\hline \multicolumn{4}{|c|}{ Left Spectrometer Arm } \\
\hline Detector & $\eta_{e}(\%)$ & $\eta_{\pi}$ & Cuts \\
\hline Gas Cerenkov & 99.9 & $>770$ & ADC sum $>400$ \\
\hline Pion Rejectors & 98 & $\approx 38$ & $\begin{array}{l}E_{P R 1}>0.42 p, E_{P R 2}>100, \\
0.75 E_{P R 1}+E_{P R 2}>0.8 p\end{array}$ \\
\hline Combined & 98 & $>3 \times 10^{4}$ & \\
\hline \multicolumn{4}{|c|}{ Right Spectrometer Arm } \\
\hline Detector & $\eta_{e}(\%)$ & $\eta_{\pi}$ & Cuts \\
\hline Gas Cerenkov & 99 & 900 & ADC sum > 342 \\
\hline Pre-Shower, Shower & 98 & $\approx 182$ & $\begin{array}{l}E_{p s h}>0.14 p, E_{s h}>100 \\
E_{p s h}+E_{s h}>0.75 p\end{array}$ \\
\hline Combined & 97 & $>1.6 \times 10^{5}$ & \\
\hline
\end{tabular}

Table 7.2: The electron efficiencies and pion rejection factors for the left and right arm spectrometers. Where $p$ refers to the central electron momentum of the spectrometer.

peaks becomes larger and easier to differentiate [41].

\subsubsection{Combined Particle Identification Efficiency}

Because the pion asymmetry is larger than the electron asymmetry and the pion/electron ratio is $\approx 1$, good particle identification was necessary for this experiment. A thorough analysis of the efficiencies of the Čerenkov and lead-glass detectors was done by Xiaochao Zheng for scattered electron momentums of $0.8<p<2.0$ $\mathrm{GeV}$ and is detailed in her thesis [41]. The scattered electron momentums of E97-103 are slightly above this, but this analysis still applies.

The particle identification efficiencies are characterized by two variables. The first, $\eta_{e}$ is the electron efficiency and is defined as the percentage of electrons that enter the detector that are detected. This is necessary to know when calculating cross sections. The second, $\eta_{\pi}$ is the pion rejection factor is the ratio of pions identified as pions to pions mis-identified as electrons. The larger this number is, the smaller the error from mis-identified pions is to the electron asymmetry. The values for these efficiencies for the left and right spectrometer are summarized in Table 7.2. 


\subsection{Data Acquisition System}

The ADC and TDC information from the detectors, helicity information and information from the beam-line apparatus are stored in data files by the Hall A data acquisition system (Hall A DAQ). The DAQ is built on CODA, which is a software package designed specifically for nuclear physics applications. There were actually three DAQ systems running: one for each spectrometer arm and one for the helicity feedback system known as the Parity DAQ.

The data files recorded by the DAQ contained three types of information. The first were events resulting from triggers of the S2 scintillator planes. These store information about the detectors and helicity information and are known as CODA events. The CODA event rate in the experiment for the DIS data was $<2 \mathrm{kHz}$. The second type of event stored contains information from the EPICS database. This database contains a large quantity of information about the spectrometer magnets, beam line apparatus, target information and other slow control information about the experiment. The third type of event put into the data files is scaler information. Scalers record the number of triggers and accumulated charge of the run. Because the scalers are read directly from the detector electronics, there are no missing events in the scalers, unlike the DAQ, which can't keep up with the data rate being produced by the detectors.

The data is first written to a local disk and then it is moved to the Jefferson Lab Mass Storage System (MSS). The MSS stores the data on tape and it can be retrieved at any time in the future for analysis. 


\section{CHAPTER 8}

\section{Asymmetry Analysis}

\subsection{Extracting Raw Asymmetries}

\subsubsection{Overview}

The longitudinal and and transverse raw asymmetries presented in Eq. 4.9 are extracted from the data files recorded by the detector package and data acquisition system. The data files are stored by the DAQ in the Jefferson Lab Mass Storage System (MSS). There is a data file for every run taken during the experiment. The run length is limited either by the number of events ( $<3$ million events) if the rates are high or by time if the rates are low (no runs longer than an hour). Each spectrometer arm has its own data file for each run.

The data files are analyzed using the software presented in Fig. 8.1 and are converted to N-tuples by ESPACE. The scaler information is also extracted from the raw data files. Information from the scalers and the N-tuples are then combined to produce charge and dead-time corrected asymmetries.

\subsubsection{Creating N-tuples with ESPACE}

ESPACE (Event Scanning Program for Hall A Collaboration Experiments) is a software application specifically written to take data files from the data acquisition system and turn them into manageable physics information. Some of the many useful tasks that ESPACE does is particle track reconstruction using the VDC and scintillator information, creating histograms of the lead-glass and C̆erenkov detector signals and constructing N-tuples which store this information [79]. N-tuples are 


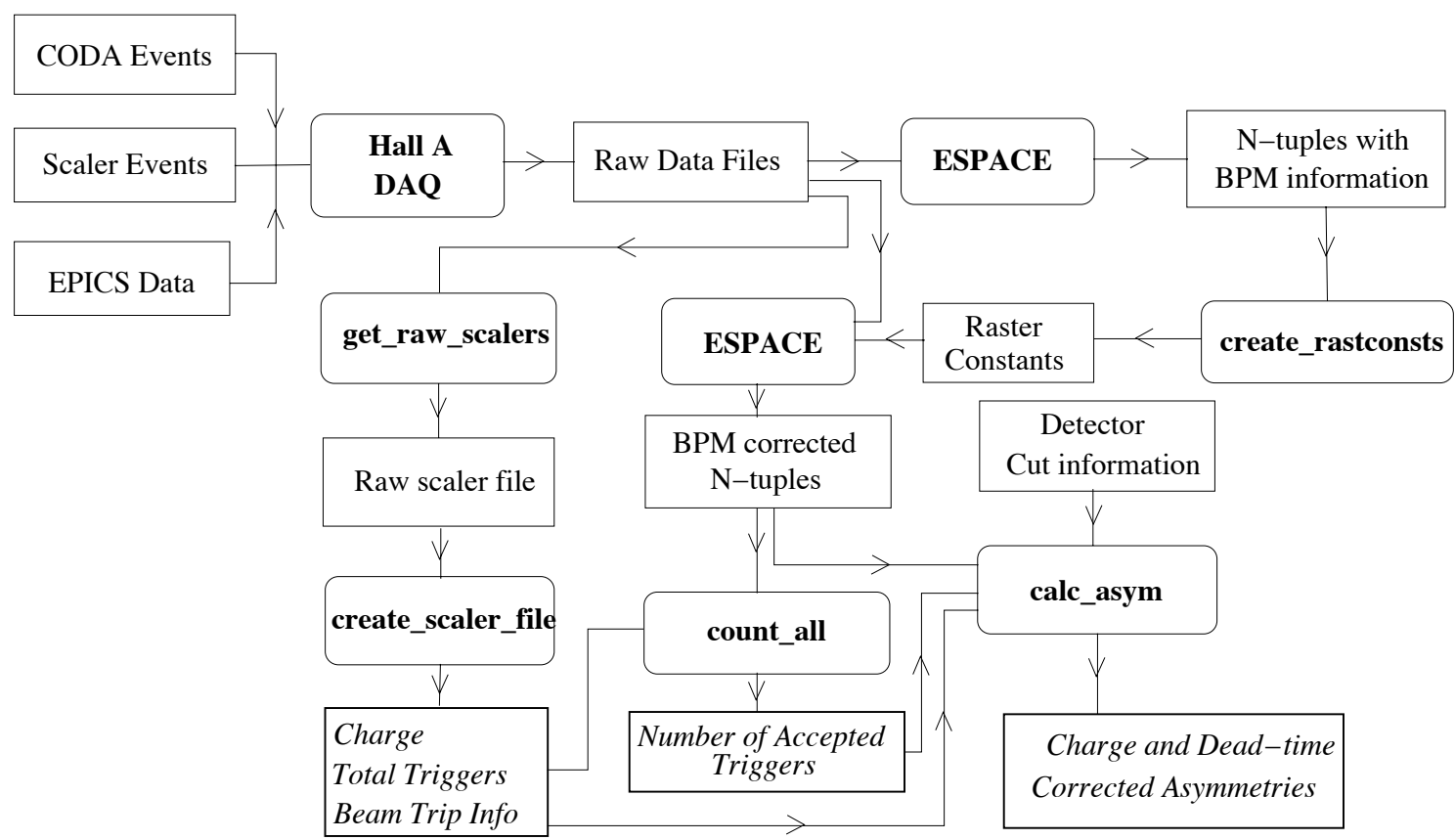

Figure 8.1: A flowchart of the various data files and analysis programs used to calculate the charge and dead-time corrected asymmetries.

sequential arrays, where each element of the array is an event with an associated set of variables. These N-tuples are created in CERNLIB HBOOK format, but are converted to a ROOT file for the purposes of this analysis.

For the E97-103 analysis, an N-tuple was made for each good run. The criteria for a good run was that it surpassed a minimum length and no comments were made concerning instability in the log books. The N-tuple was made in a series of stages. The first N-tuple made from ESPACE, as shown in Fig. 8.1, was made to extract raster and BPM information from the raw data files. The reconstruction of the scattering location requires average values of the spot position and raster size and this can only be done by making a complete pass of the data file. This N-tuple containing the correct BPM and raster information is analyzed using a ROOT $\mathrm{C}++$ code known as create_rastconsts. The output of create_rastconsts creates the necessary input file for ESPACE to do the scattering point reconstruction correctly.

After the beam position input files are created, ESPACE is re-run and the new 
N-tuples contain information about the reconstructed momentum, scattering point and direction information of every event, values for the Čerenkov and lead-glass detectors and the event time. Though ESPACE is capable of doing elaborate cuts on the data, all events recorded by the DAQ are included in this N-tuple.

\subsubsection{Extracting Charge and Dead-time Information}

The scaler information used to calculate the helicity correlated charge and deadtime corrections is extracted from the raw data files. The scaler data is sent to the DAQ every four seconds. The information from the scalers relevant to this analysis are the total number of $T_{1}, T_{2}, T_{3}$ and $T_{4}$ triggers for the positive and negative helicity states, the charge accumulated in BCM with the $3 \mathrm{x}$ amplification for both positive and negative helicity states and the accumulated time from the $1024 \mathrm{~Hz}$ clock for positive, negative and ungated helicity. In the experiment preceding E97-103, The $T_{1}$ and $T_{3}$ trigger scaler that was normally used started to show signs of multiple triggers for one event. Another set of scalers were created called $T_{1}$ clean and $T_{3}$ clean that did not have this problem [80]. Whenever the total number of triggers is mentioned, these quantities are what is used. A ROOT $\mathrm{C}++$ program was written called get_raw_scalers that reads every scaler event in the raw data file and outputs out a text file with the scaler values for each scaler event.

Since the scalers are normally cleared at the beginning of the run, the last reading of the scalers can be used to represent the accumulated values of the scaler variables over the run. However, there are two reasons the scalers aren't treated this way in this analysis. The first reason was that there were times, specifically during the carbon quasi-elastic data taking, that the scalers were not being cleared at the beginning of the run [81]. The second reason is that there was concern about large charge asymmetries during the ramping of the beam current. To study the effect of this beam ramping, it was useful to be able to cut out parts of the run. This meant 
that one had to be able to subtract the proper amount of triggers and charge that occured during the removed periods from each of the accumulated scalers.

The scaler information was summed by a ROOT $\mathrm{C}++$ program known as create_scaler_file. This program calculated the total scaler values by calculating the increase in the accumulated value of each variable for each 4 second interval between scalers. This can be described by:

$$
s_{\text {sum }}=\sum_{i=2}^{N} \Delta s_{i}
$$

where $s_{\text {sum }}$ is the accumulated value for a particular scaler variable $s, N$ is the number of scaler events and $\Delta s_{i}$ is defined by:

$$
\Delta s_{i}= \begin{cases}s_{i}-s_{i-1} & \text { if } I_{i} \geq I_{\min } \\ 0 & \text { if } I_{i}<I_{\min }\end{cases}
$$

where $s_{i}$ is the value of scaler $s$ for scaler event $i, I_{\min }$ is the minimum current and $I_{i}$ is defined as:

$$
I_{i}=\frac{Q_{i}^{+}+Q_{i}^{-}-Q_{i-1}^{+}-Q_{i-1}^{-}}{t_{i}^{u}-t_{i-1}^{u}}
$$

where $Q_{i}^{ \pm}$is the charge scaler value for event $i$ and helicity \pm and $t_{i}^{u}$ is the value of the ungated helicity clock value for event $i$. The values for $Q^{ \pm}$must be calculated using the calibration constants in Eq. 5.7. The value for $I_{\min }$ is arbitrary and the change in the raw asymmetry due to change in $I_{\min }$ will be discussed later. Whenever an interval of the scaler information is cut out, the clock times are recorded for that interval relative to the first clock time. This is done so that the events in the N-tuples from that interval can be removed as well.

The charge values and total triggers have been acquired from the scaler information in the raw data file. The only other information needed is the total accepted triggers by the data acquisition system. This number is extracted by counting all events in the N-tuple output from ESPACE with the ROOT C++ program count_all. If beam ramping cuts are made, the events corresponding to 


\section{Scaler time over Event Time}

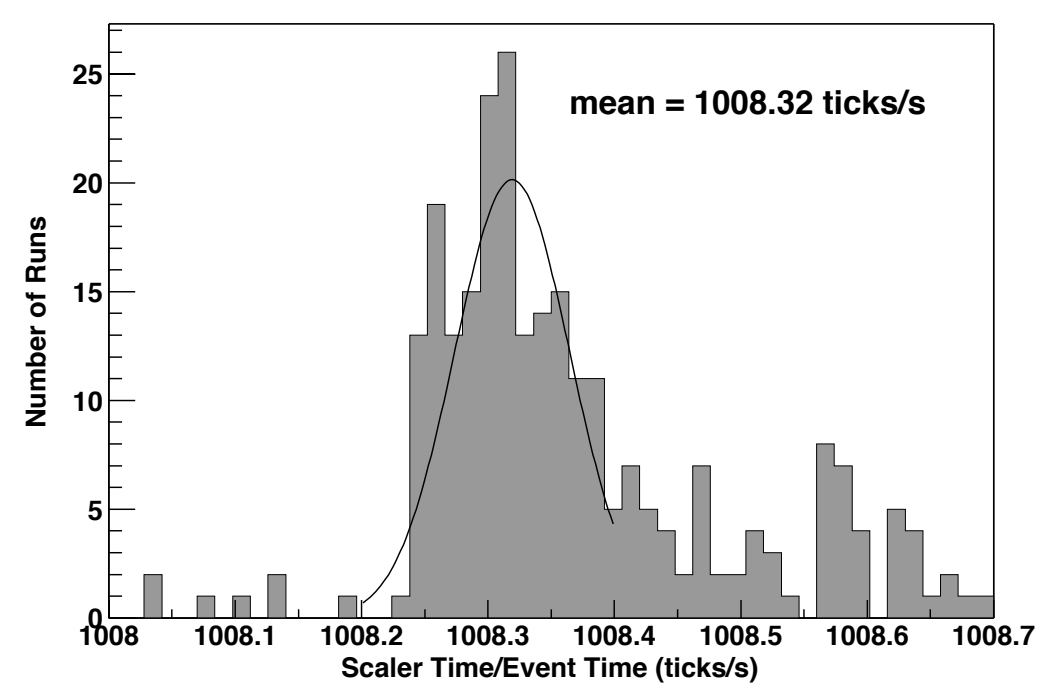

Figure 8.2: A histogram of the ratio of total scaler clock time to event clock time of a run.

these times are removed. This is done by looking at the event_time variable in each event. However, the scaler clock's unit is a tick of the $1024 \mathrm{~Hz}$ clock where event time from the N-tuple is in seconds. To convert from one to the other, one can use:

$$
t_{\text {scaler }}=(1008.32 \text { ticks } / \mathrm{s}) t_{\text {event }}
$$

where $t_{\text {scaler }}$ is the time on the scaler clock in clock ticks and $t_{\text {event }}$ is the time assigned to events in seconds. This constant is determined by looking at data files and their corresponding scaler files. The ratio of the scaler time to the event time for the same run is plotted in Fig. 8.2. The reason this isn't exact is the event time is only known when an event occurs. If no events are happening there can be a gap at the end of the run, which explains why sometimes the ratio is larger than the mean. Also, there were instances where events happened after the scaler clock stopped. It is unclear whether these events were included in the accumulated scalers and assigned the wrong event time or not. In any case, varying this constant did not change the raw asymmetry significantly. 


\begin{tabular}{ll}
\hline \hline Variable & Description \\
\hline evt_type & the trigger type of event $(1,2,3,4)$ \\
ev_time & the time of the event from the start of run \\
ntrack & number of tracks from VDC reconstruction \\
react_z & scattering location on target cell axis \\
tg_ph & horiz. scattering angle w. r. t. central angle of HRS \\
tg_th & vert. scattering angle w. r. t. central angle of HRS \\
tg_dp & relative difference in momentum w. r. t. central momentum of HRS \\
beta & the velocity of the particle over $c$ \\
psh & pre-shower or the 1st layer of pion rejector value \\
sh & shower or 2nd layer of pion rejector value \\
\hline \hline
\end{tabular}

Table 8.1: The variables used to find good electron events.

\subsubsection{Analyzing N-tuples}

The values of $N^{+}$and $N^{-}$in Eq. 4.9 are extracted for each run from the Ntuple output from ESPACE. This process consists of counting the number of good electrons of each helicity within the same spectrometer acceptance. The decision whether or not the event is an electron and that it lies within certain acceptance criteria is based on the values of the reconstructed variables associated with that event. The variables used to make that decision are listed in Table 8.1.

There are four types of cuts made on events: good trigger cuts, good electron cuts, acceptance cuts and event time cuts. Good trigger cuts are those made on trigger type and VDC tracking. Only events $T_{1}$ or $T_{3}$ (evt_type==1 II evt_type==3) and one good event track (ntrack==1) were considered good track events. Good electron cuts use the Čerenkov and lead glass detectors variables (cer,psh, sh) to separate electron events from pion events. In addition, beta is used to separate particles by velocity, which is an effective way of getting rid of cosmic rays that have $\breve{C}$ erenkov and lead-glass detector signals. Acceptance cuts use the reconstructed scattering variables (react_z,tg_ph,tg_th,tg_dp) to keep electrons that are close to the physics kinematics and to exclude ones that differ from them significantly. Since the spectrometer optics aren't well known through the whole acceptance, it is 
also important to keep only electrons that travel through an understood part of the spectrometer. Event time cuts come from the scaler analysis and are used to remove events during low current periods and when the scaler is not recording events.

All the asymmetry analyses, including physics production, false asymmetry and elastic asymmetry uses these same techniques and software to create N-tuples and calculate the output values needed to calculate $A_{\text {raw }}$.

\subsection{Establishing Sign Convention}

The sign of the asymmetry calculated by this procedure needs to match the sign of the world data. To do this a known longitudinal asymmetry and a known transverse asymmetry are measured. The sign of the longitudinal asymmetry is measured using polarized ${ }^{3} \mathrm{He}$ elastic scattering. The sign of the transverse asymmetry was measured using the polarized $\Delta(1232)$ resonance which has a large transverse asymmetry. The sign convention was established by data taken in the E99-117 experiment (which directly preceded E97-103) and is described in the thesis of Xiaochao Zheng [41]. The results of that analysis were used in E97-103 and are presented in Table 8.2. An important point in the asymmetry analysis is that sign of the longitudinal asymmetry is the same in both arms, while the sign of the transverse asymmetry is different in the the two arms.

There were 12 target, beam and spectrometer combinations in E97-103 listed in Table 8.2. There were 4 combinations that measured the longitudinal asymmetry. These correspond to a target polarization direction of $0^{\circ}$. In principle, additional data could be taken with the target polarization at $180^{\circ}$, but the half-wave plates could not be aligned for the longitudinal and transverse lasers at the same time. Since the experiment ran much longer with the transverse lasers, it was decided not to use the $180^{\circ}$ polarization direction. The other 8 configurations were used to measure the transverse asymmetry. Measuring the same quantity in multiple 


\begin{tabular}{|ccc|c|}
\hline \hline $\begin{array}{c}\text { Beam 1/2 } \\
\text { wave plate }\end{array}$ & $\begin{array}{c}\text { target pol. } \\
\text { direction }\end{array}$ & HRS & $\begin{array}{c}\text { sign } \\
\text { convention }\end{array}$ \\
\hline OUT & 0 & left & positive \\
OUT & 0 & right & positive \\
IN & 0 & left & negative \\
IN & 0 & right & negative \\
OUT & 90 & left & positive \\
OUT & 90 & right & negative \\
IN & 90 & left & negative \\
IN & 90 & right & positive \\
OUT & 270 & left & negative \\
OUT & 270 & right & positive \\
IN & 270 & left & positive \\
IN & 270 & right & negative \\
\hline \hline
\end{tabular}

Table 8.2: Sign convention used for asymmetries during E97-103 for the various beam and target polarization combinations. No data was taken in the $180^{\circ}$ target polarization direction in E97-103.

configurations is a powerful tool for eliminating false asymmetries.

\section{$\underline{8.3 \quad \text { Detector Cut Studies }}$}

\subsubsection{Electron Identification Studies}

Cuts on the Cerenkov and lead-glass detectors separate scattered electrons from other types of charged particles. The goal of the cuts is to maximize the number of scattered electron events, while minimizing the amount of unwanted events. Usually, it is beneficial to err on the side of cutting out good electrons rather than let a significant number of unwanted events into the asymmetry analysis.

There are only two cuts done on the Čerenkov ADC sum values for each event as shown in Fig. 8.3. There is a ČCenkov cut minimum that does most of the work removing pions. This cut is usually on the left hand edge of the large electron peak. The small peak on the left of the Čerenkov peak are $\delta$-electrons and need to be cut out. The second cut on the C̆erenkov is a maximum value cut. Its purpose is to cut out some bad events in the top ADC channel. This cut can be left out with no 


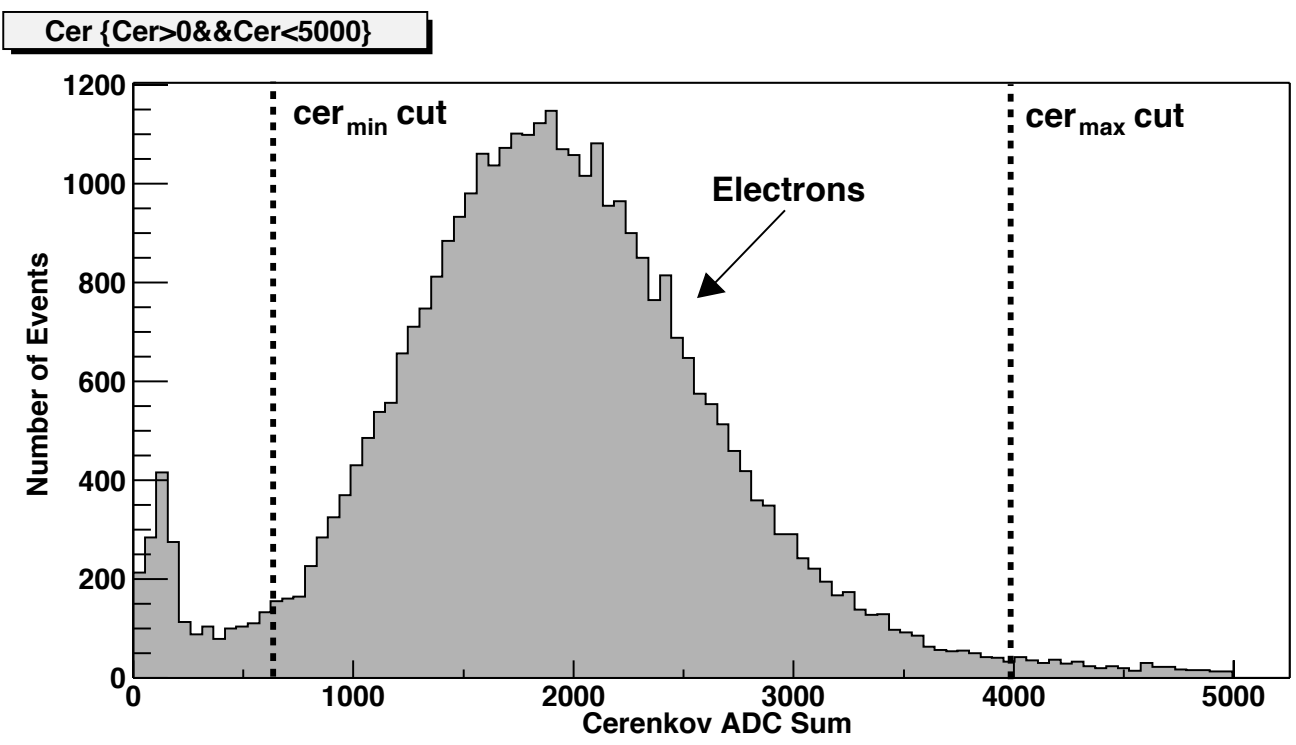

Psh:Sh $\{P s h>0 \& \& P s h<4000 \& \& S h>0 \& \&$ Sh $<2500\}$

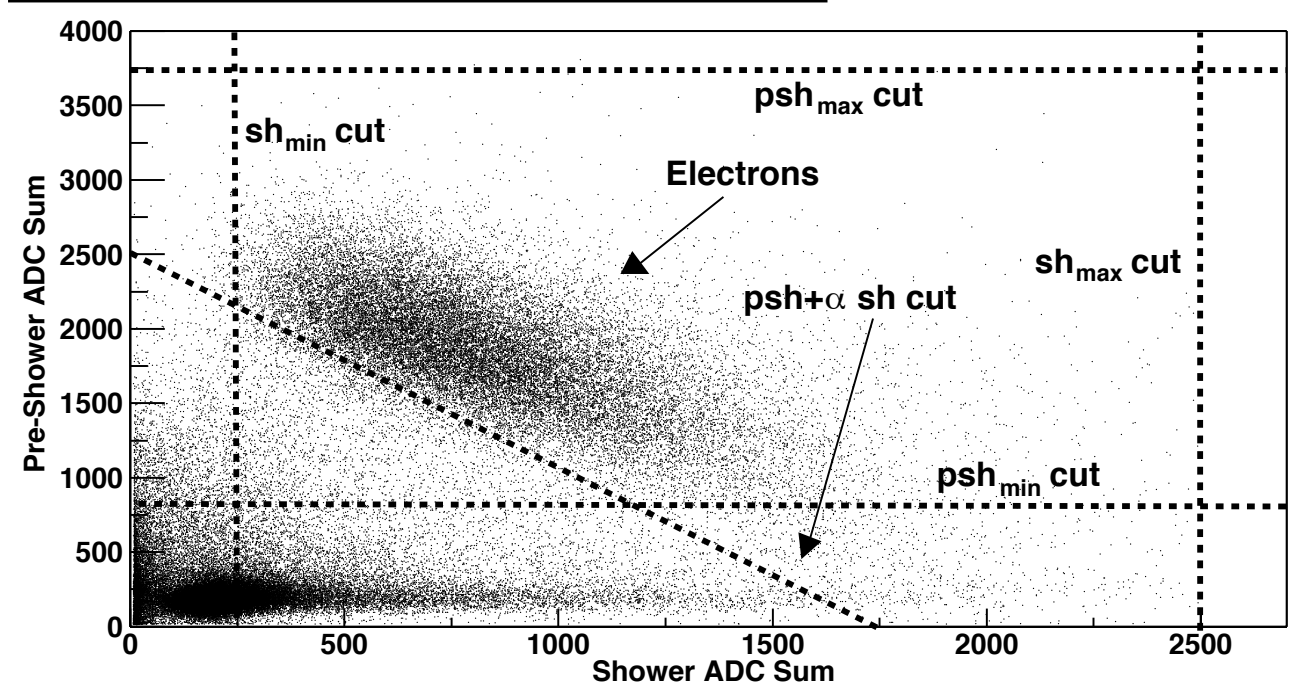

Figure 8.3: A histogram of the C̆erenkov ADC sum for a set of events and a 2-D plot of pre-shower and shower $A D C$ sums. Examples of cuts used in the asymmetry analysis are shown. 


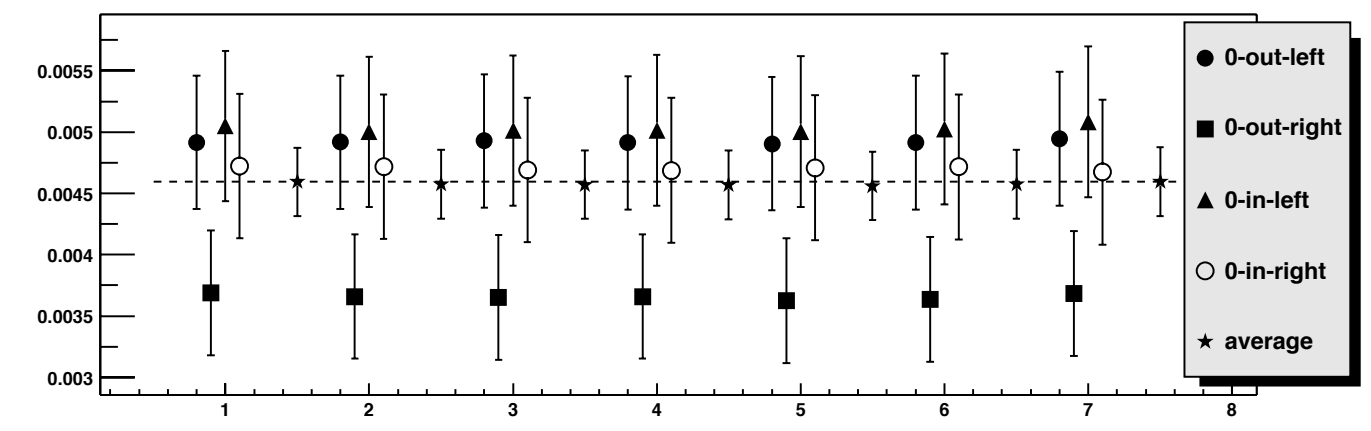

$Q^{2}=0.58$ - Perpendicular Asymmetry - Cerenkov Cut Study

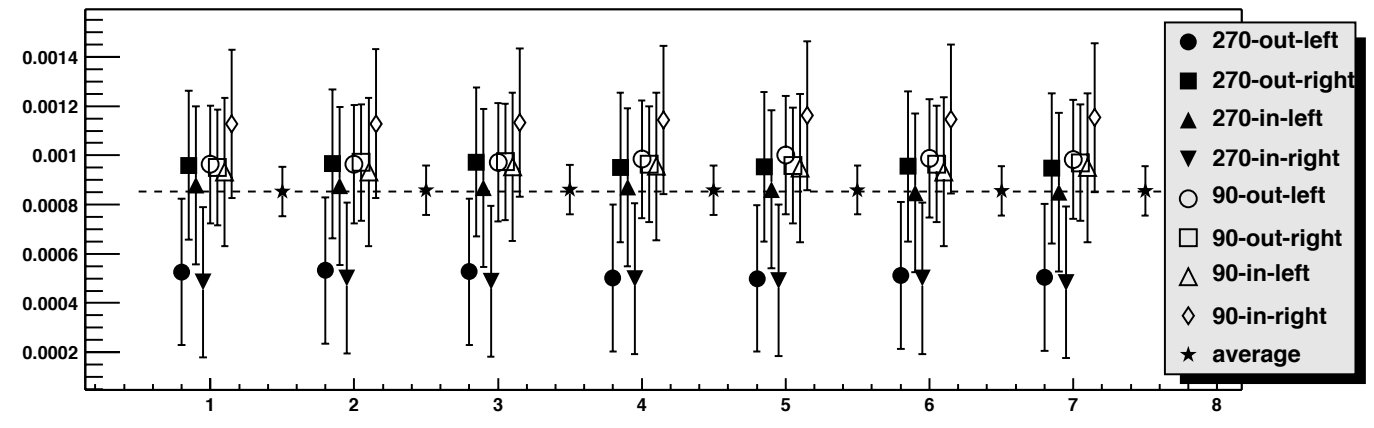

Figure 8.4: A plot of the effect of different Cerenkov cuts on the physics asymmetry. The top plot is the effect of different cuts on the four longitudinal configurations and their average. The bottom plot is the effect on the eight transverse configurations and their average. The cuts become tighter from left to right.

effect on the asymmetry.

The goal of the pre-shower and shower cuts (or pion-rejector layer 1 and 2 cuts, referred from here on out as left arm pre-shower and shower cuts for convenience) is to cut out everything but the big blob of electrons in the middle of the lower plot in Fig. 8.3. Both the pre-shower and shower have a minimum and maximum cuts to cut out unwanted events. There is an additional 2-D cut (referred to a psh $+\alpha \mathrm{sh}$ cut on the plot) that is parallel to and set on the edge of the electron blob.

The effect of changing these cuts has been studied. The asymmetry of all configurations and kinematics were calculated and plotted. An example of one of these studies is shown in Fig. 8.4. In that plot, the Cerenkov cut was changed from 500 to 1000 with no shower or pre-shower cuts at all and leaving all the acceptance 
cuts the same. In all the studies of the particle identification, it was found that as long as there was a cut on the C̆erenkov detector above zero the asymmetry did not change significantly, even if there were no other cuts. This means that the Cerenkov detector was efficient enough by itself within the error bars of the raw asymmetry. However, the final analysis uses more conservative cuts on the Cerenkov and leadglass detectors to err on the side of caution.

\subsubsection{End Window Cut Studies}

The end windows of the polarized ${ }^{3} \mathrm{He}$ glass cells are a source of large quantity of scattered events. Not being events from ${ }^{3} \mathrm{He}$, they need to be removed. Tight cuts on the electron scattering variable react_z will cut out most of the scattering from the windows. To do this each end window was fit with a Gaussian along react_z. The cuts were made $3 \sigma$ away from the mean of the Gaussian as shown in Fig. 8.5. Empty reference cell runs were taken to estimate the amount of background from the end windows, but because there were some residual gases in the reference cell it was difficult to estimate the background from the windows with $3 \sigma$ cuts. A conservative estimate is that $<2 \%$ of the events used in the asymmetry analysis come from the end windows.

\subsubsection{Acceptance Cut Studies}

Different cuts on the spectrometer acceptance variables were also studied to see the effect on the asymmetry. The cuts on the acceptance variables, as shown in Fig. 8.6, are all done in two dimension to maximize the size of the acceptance, while still cutting out unwanted events. The acceptance cut studies calculated the asymmetry starting with a loose cut and moving to a tight cut.

The study plotted in Fig. 8.7, shows the electron asymmetry for all configurations and the average with 8 gradually tightening cuts on tg_ph and react_z. 


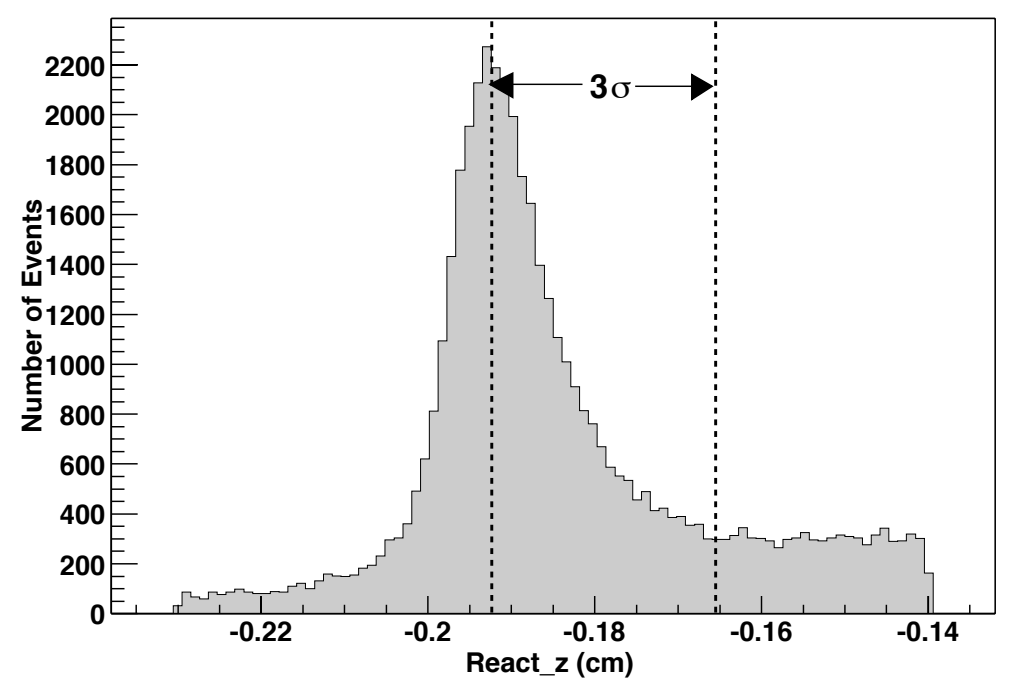

Figure 8.5: A histogram of scattering position along the target cell axis showing the end window cut.

This study was repeated with a tight cut on tg_th and tg_dp. Then the same sort of study was done on tg_th and tg_dp with loose and tight cuts on tg_ph and react_z. All of these studies were repeated for the pion asymmetry and for all 5 kinematic settings.

From all of these studies no significant trends in the data were found. The average of the configurations never deviated from the average of the loosest cut by more than one $\sigma$ for any of the cut studies. Often, a single configuration would deviate from the other configurations, but never more than two $\sigma$ and never consistently through different kinematics. A configuration that deviated from average in the electron asymmetry would stay with the average in the pion asymmetry. Because of the consistency of the values for the asymmetry, the loosest cuts were chosen to calculate the asymmetry to minimize the statistical error of the experiment. 

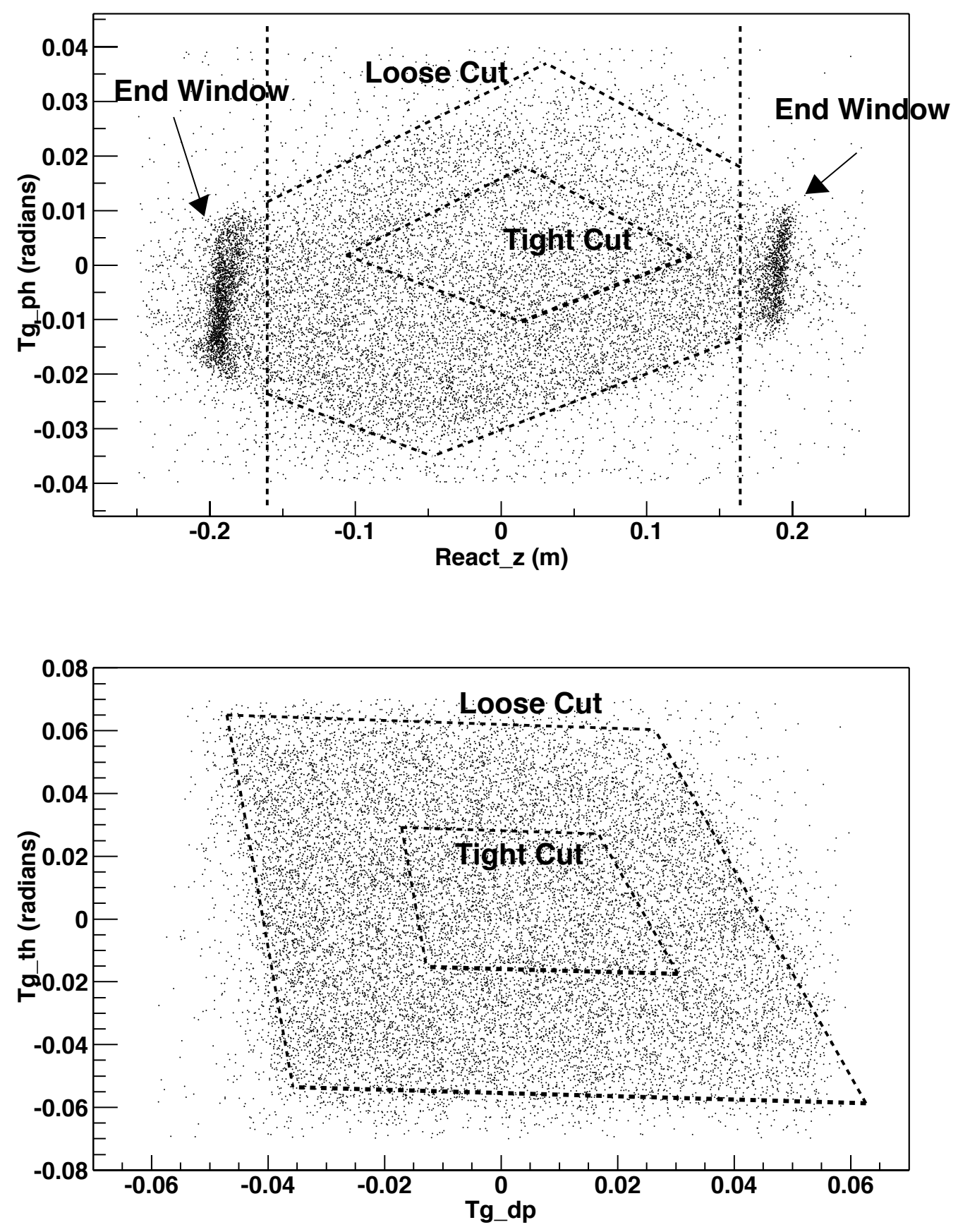

Figure 8.6: Two plots of the spectrometer acceptance variables. The top plot is of the horizontal scattering angle $\phi$ vs. scattering point along the cell axis, react- $z$. The bottom plot is of the vertical scattering angle $\theta$ vs. the relative momentum difference of the event from the central momentum. 

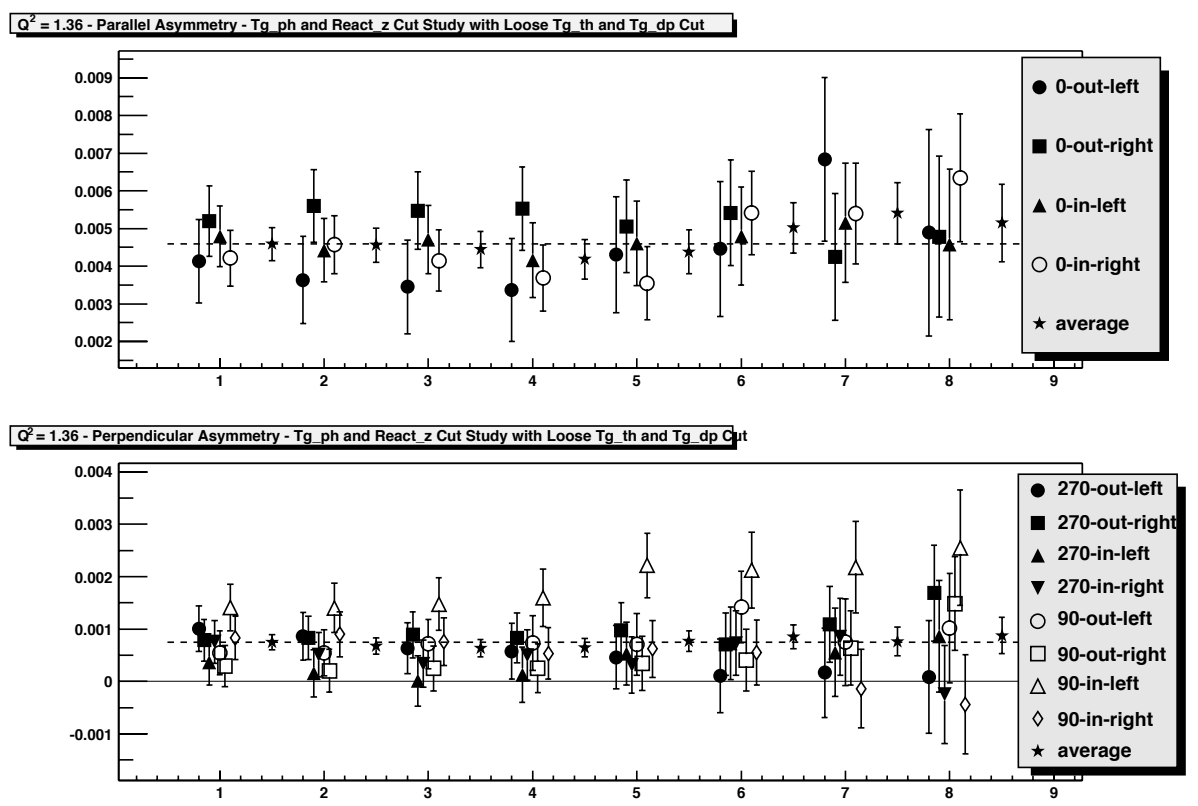

Figure 8.7: A study of the effect of tightening cuts along the horizontal scattering axis and the target chamber axis. The dashed line is the value of the asymmetry with the loosest cuts.

\section{$\underline{8.4 \quad \text { False Asymmetries }}$}

\subsubsection{Removing Beam Ramping Periods}

Because of the high statistics taken in the transverse polarization configuration, the experiment is potentially sensitive to false asymmetries. One false asymmetry of concern was the effect of ramping the beam on the charge asymmetry. Because of the thin end windows on the target cell, whenever the beam is first turned on, the current is ramped up slowly to avoid a thermal shock that could lead to a cell rupture. These beam ramping periods generally last around 30 seconds. During the experiment, it was noticed that the charge asymmetry was becoming much larger during these beam ramping periods [82]. If the charge asymmetry during these periods was large enough, the charge correction would not be enough to compensate for them.

To study this the phenomena, the software described in section 8.1.3 was used to cut out periods in the data where the average current was below either $1 \mu \mathrm{A}$ 

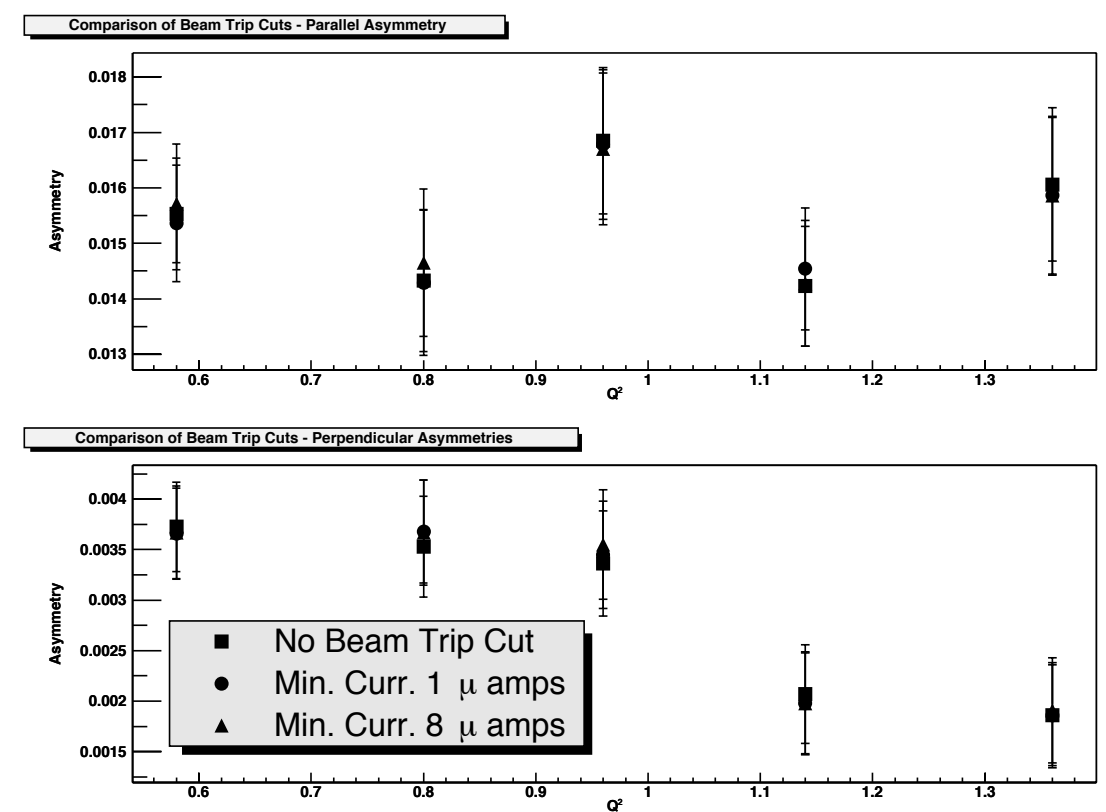

Figure 8.8: A comparison of the physics asymmetries with different cuts on the minimum current.

or $8 \mu A$. This was compared to a calculation of the asymmetry where no cuts on the beam current were made. The results are shown in Fig. 8.8. Obviously, the effect of cutting the beam ramping either at the $1 \mu \mathrm{A}$ or $8 \mu \mathrm{A}$ level is not significant compared to the statistical errors of the experiment. In the final asymmetry analysis the beam current minimum was set to $1 \mu A$ to cut current levels that were out of range for the $3 \mathrm{x}$ gain amplifier of the BCMs.

\subsubsection{The Effect of Holding Field Misalignment}

Another source of false of asymmetry was mixing of the longitudinal and transverse asymmetry due to slight misalignment of the field. Because the longitudinal asymmetry was significantly larger than the transverse asymmetry, a misalignment might shift the transverse asymmetry. Measurement of the holding field was done as described in section 6.8.3. The direction surveys of the holding field mostly agreed, but disagreed $0.5^{\circ}$ in the $90^{\circ}$ configuration. The two surveys of most interest is survey 3, which took place right before E97-103 and survey 4 which happened just 

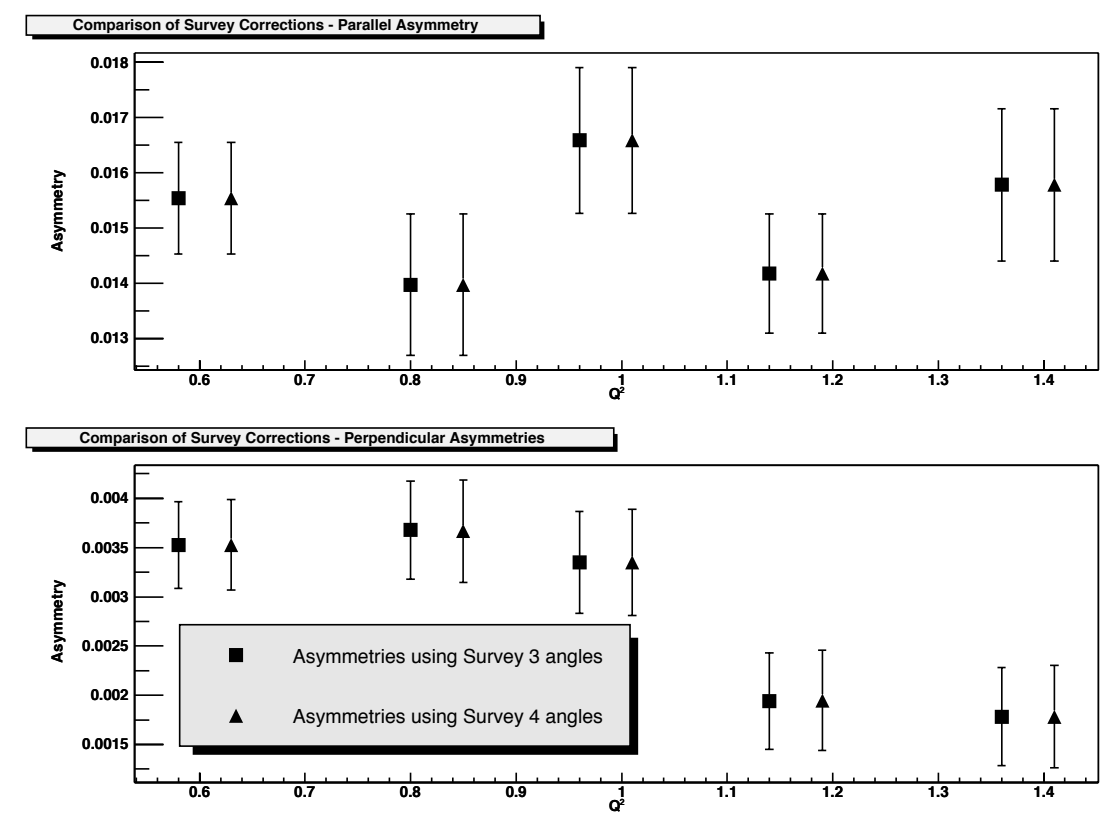

Figure 8.9: A comparison of the physics asymmetries correcting the field alignment with the compass surveys. The $Q^{2}$ values of the fourth survey have been shifted slightly so they can be seen.

after. Survey three was used to set the holding field angles in the experiment.

The effect of the discrepancy of the compass surveys on the physics asymmetries is shown in Fig. 8.9. The effect of correcting the asymmetries for the difference in compass surveys is negligible. This is because the longitudinal asymmetry doesn't change sign between left and right spectrometer arm and the transverse asymmetry does. Therefore, when a longitudinal asymmetry appears when the target is tranversly polarized, it will cancel when the all the different configurations are added together. This is only the case, and it was the case in E97-103, if there is roughly equal amount of data in both spectrometer arms for each configuration.

\subsubsection{Carbon Quasi-Elastic Analysis}

In an effort to measure false asymmetries, two days were spent running on the carbons foil target at $1.197 \mathrm{GeV}$ at quasi-elastic kinematics $\left(E^{\prime}=1.123 \mathrm{GeV}\right.$ and $\left.\theta=18.6^{\circ}\right)$. Since the carbon foils are unpolarized and the parity violation 


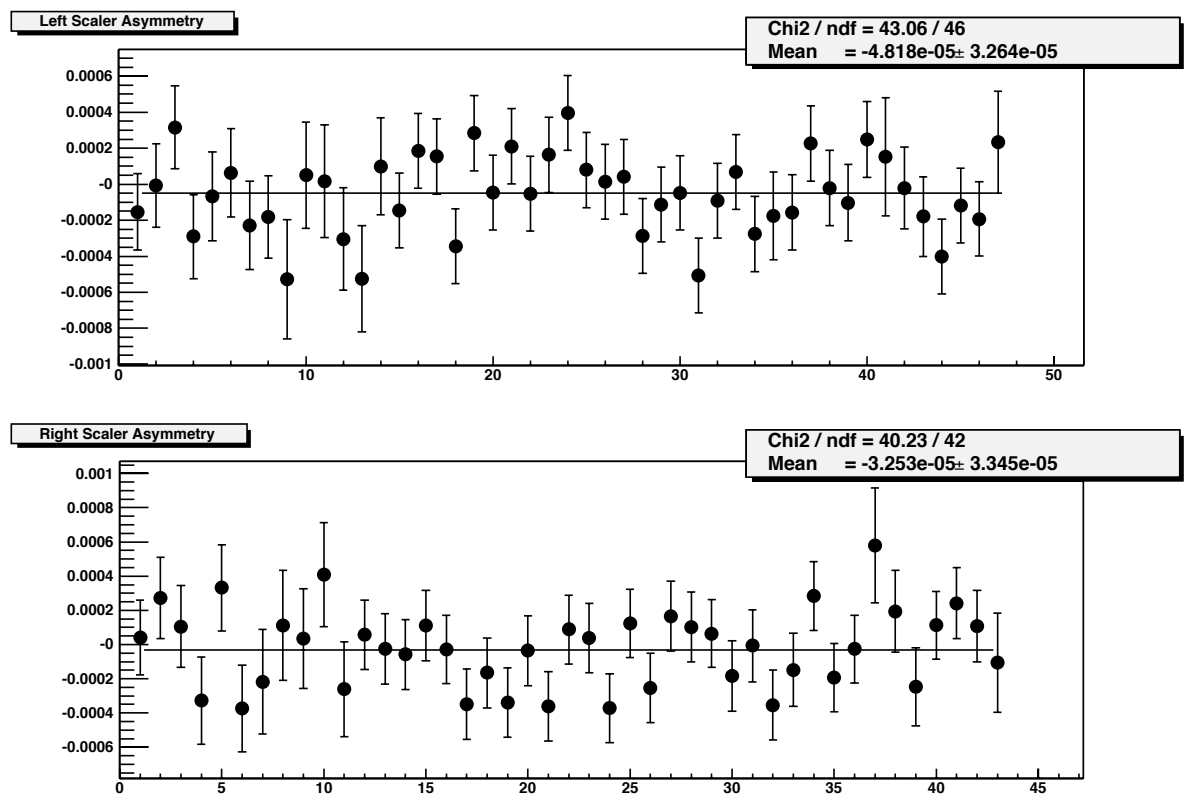

Figure 8.10: The asymmetry in the left and right spectrometer arm during carbon quasielastic running using charge corrected scaler triggers.

asymmetry is insignificant at this level of statistical error, the asymmetry measured from this data should be consistent with zero.

The carbon quasi-elastic asymmetry was measured in two ways. The first way was to measure the charge-corrected asymmetry of the scaler $T_{1}$ and $T_{3}$ triggers. This has the advantage of higher statistics, but since the physics asymmetry is measured using detector events it is an incomplete measure of the false asymmetry. The results of this method are shown in Fig. 8.10. The second way was to measure the asymmetry with detector events with minimal cuts. All that was required for an event to be accepted was that the event had a $T_{1}$ or $T_{3}$ trigger and a unique VDC track. The asymmetry is corrected for charge and dead-time in the usual way. The results from this method are shown in Fig. 8.11.

The false asymmetry data from the detector method was pre-scaled by a factor of 10 to keep from overburdening the Hall A DAQ. This creates complications in calculating the statistical error. The statistical error calculated using the number 

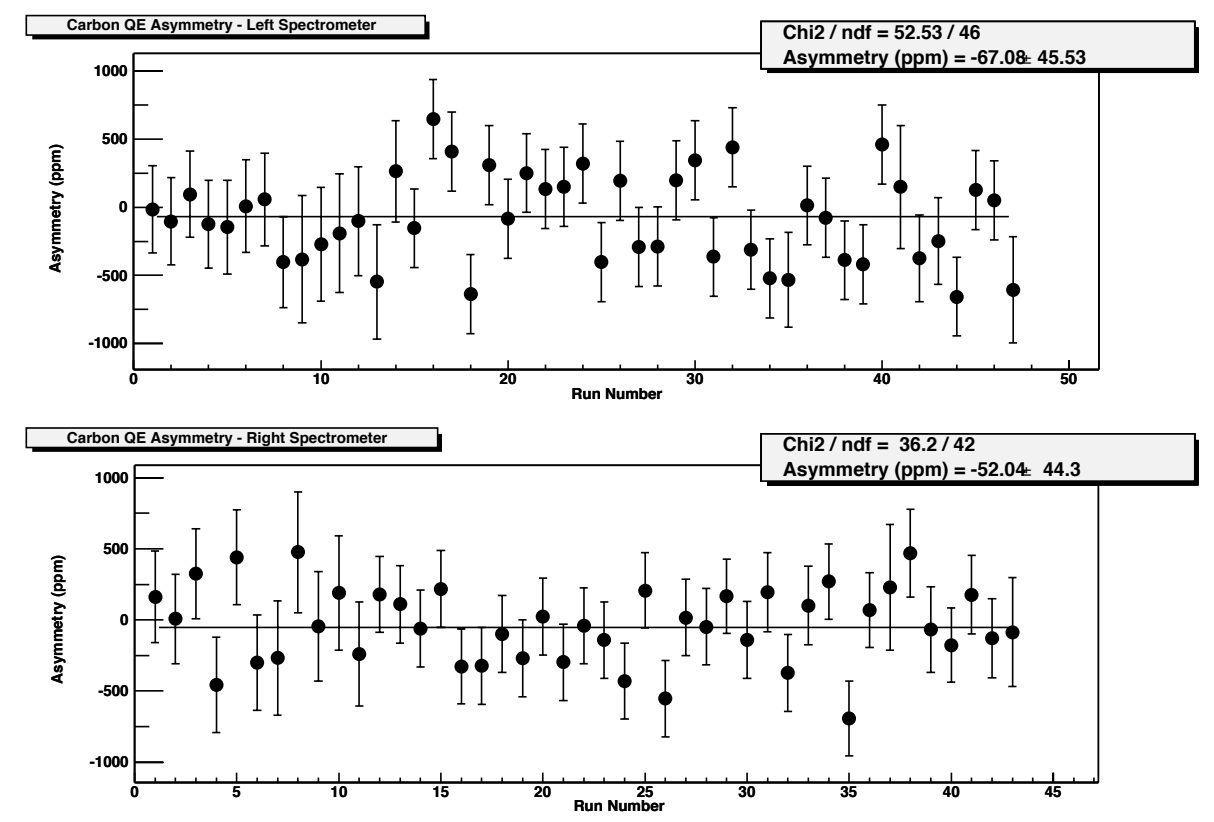

Figure 8.11: The asymmetry in the left and right spectrometer arm during carbon quasielastic running using charge and dead-time corrected detector events.

of detector event results in a $\chi^{2}$ much smaller than 1 , meaning that the error bars are too large. On the other hand, multiplying the number of events by the pre-scale factor creates a $\chi^{2}$ much larger than 1 , meaning the error bars are too small. If there were no dead-time and no cuts on the data this would be the correct way to calculate the error bars. However, since there are both dead-time corrections and cuts on the data this method can't be used. An easy, but perhaps not completely accurate way of assigning error bars to this data set is to multiply the error bars by a correction factor until the $\chi^{2}$ is on average 1 in both spectrometer arms. This has some validity since the error is statistical and ultimately whatever method used to calculate the error would have to have a $\chi^{2} \approx 1$.

Both methods of measuring the false asymmetry are within one $\sigma$ away from zero. The false asymmetry from the scalers is smaller than the false asymmetry from the detector events data, providing evidence that the false asymmetry in the detector events data is a random deviation and not a true false asymmetry. However, 
there is no way to tell if that is true and the detector events results are used for the value of the false asymmetry from carbon.

\subsubsection{End Window False Asymmetries}

The physics asymmetries are measured in a way to cancel false asymmetries. This is seen in the holding field alignment where a false asymmetry on the order of the carbon false asymmetry canceled because of changing the sign of target and beam polarizations and the two symmetric spectrometer arms. Therefore, any false asymmetry that could enter the physics asymmetry would have to be correlated with these configuration changes.

Another method of measuring the false asymmetries is to measure the asymmetry from scattering off the glass end windows of the cell. This has the advantage of taking data in a manner similar to the physics asymmetry data and can be combined together in the same way. Any false asymmetry that survives will be correlated to the configuration changes. However, the difficulty is getting enough statistics from the end window scattering.

The asymmetry results of end window scattering are shown in Fig. 8.11. The statistical error is not as good as the carbon study and could be improved by widening the cuts on the end windows; however, this creates considerable uncertainty from an unknown amount of polarized ${ }^{3} \mathrm{He}$ contamination. The events accepted in this study include all events outside the middle of the end window peak in react_z.

\subsubsection{Calculating Error from False Asymmetry}

Interpreting the error from the carbon and end window false asymmetry data is not straight forward. The most relevant result would be from the end window false asymmetry measurements since this asymmetry measures the false asymmetry correlated with the configuration changes. However, the measurement presented 


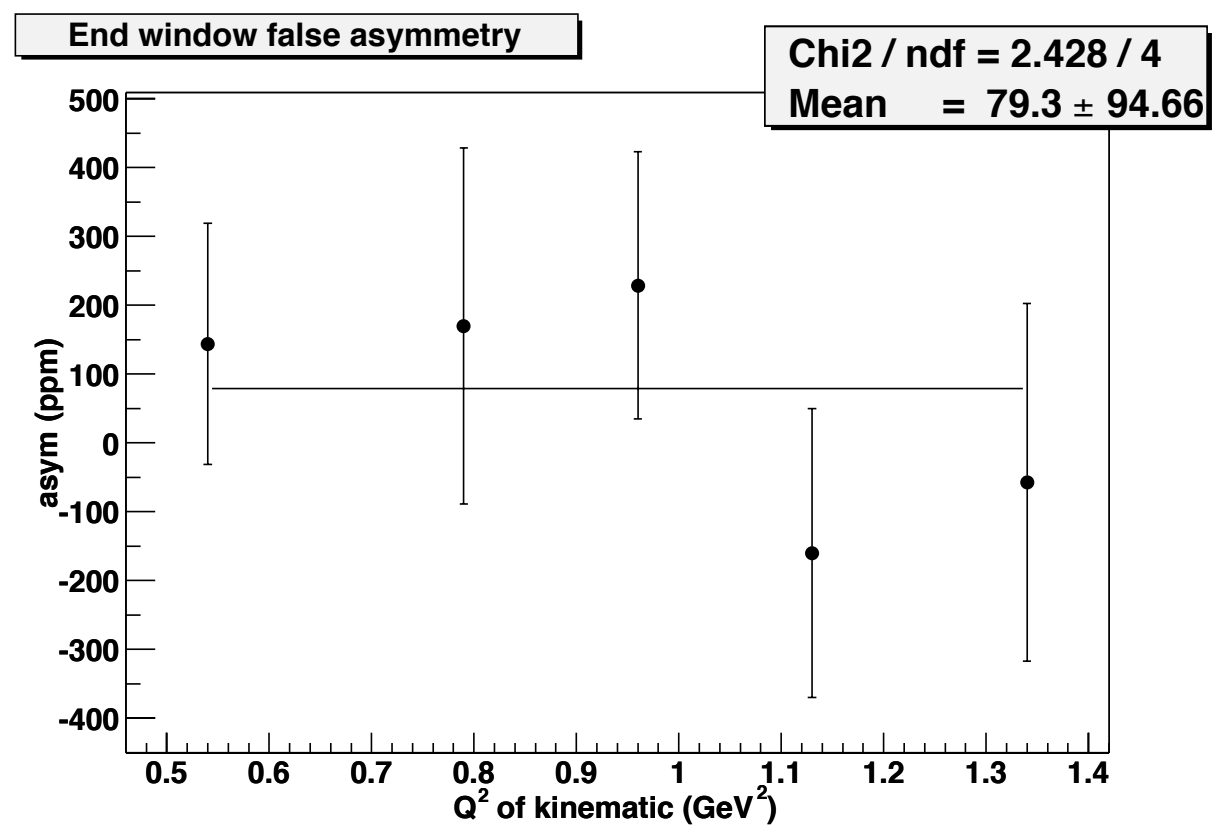

Figure 8.12: The asymmetry calculated from accepting only events from the glass end windows.

here is statistically limited and there would always be considerable uncertainty from contamination from polarized ${ }^{3} \mathrm{He}$ scattering. On the other hand, the results from scalers and detector events from the carbon quasi-elastic scattering are more statistically accurate, but any false asymmetry they would measure would cancel using the configuration changes.

It was decided to use $60 \mathrm{ppm}$ as the false asymmetry error on the physics asymmetries, which is the average of the asymmetry in both spectrometer arms in the detector event analysis from carbon quasi-elastic scattering. This was chosen because this was a reasonable estimate of how well the false asymmetry was measured.

\subsection{Nitrogen Dilution}

\subsubsection{Using the Reference Cell to Measure the Nitrogen Contribution}

The polarized ${ }^{3} \mathrm{He}$ cells contain a small amount of nitrogen to improve the rubidium polarization in the pumping chamber. Events scattering from nitrogen molecules can't be separated from those scattered from ${ }^{3} \mathrm{He}$ using detector infor- 


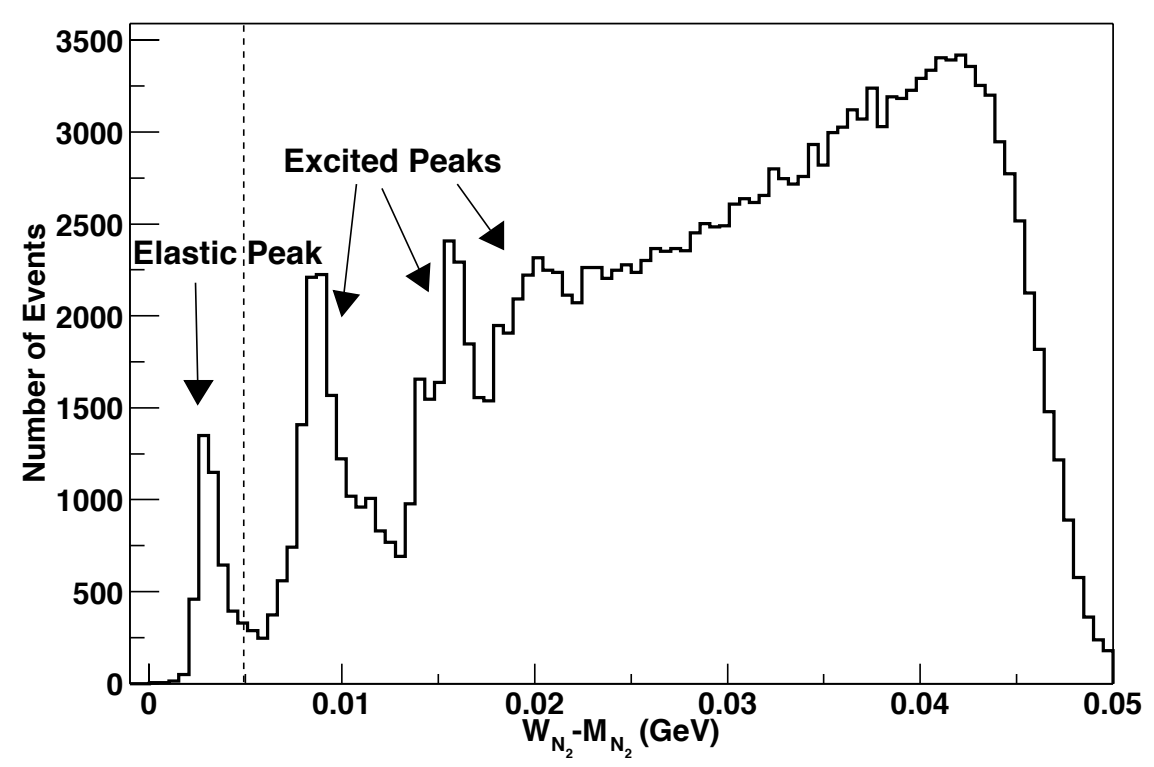

Figure 8.13: A plot of $W_{\mathrm{N}_{2}}-M_{\mathrm{N}_{2}}$ for a nitrogen reference cell run.

mation in the deep inelastic scattering regime. Since the nitrogen cross-section is larger than the ${ }^{3} \mathrm{He}$ cross-section, it contributes significantly to the unpolarized cross-section, diluting the asymmetry.

An attempt to measure the amount of nitrogen in the polarized ${ }^{3} \mathrm{He}$ cell was made using data taken on nitrogen in the reference cell. This was done at ${ }^{3} \mathrm{He}$ elastic kinematics so the elastic peak from nitrogen is in the acceptance. The nitrogen elastic peak can be clearly seen in the plot of $W_{\mathrm{N}_{2}}-M_{\mathrm{N}_{2}}$ in Fig. 8.13. This plot can be compared to the plot in Fig. 8.14 which is a set of data with the same cuts and kinematics, but on a polarized ${ }^{3} \mathrm{He}$ cell. The elastic peak of nitrogen is clearly separated from the ${ }^{3}$ He elastic peak.

By comparing the yield from the elastic peak in the reference cell, which has a known number density, to the yield from the elastic peak in a polarized ${ }^{3} \mathrm{He}$ cell the number density in the polarized cell can be determined. The method used in this analysis is:

$$
n_{\text {pol. cell }}=\frac{Y_{\text {pol. cell }}}{Y_{\text {ref.cell }}} n_{\text {ref. cell }}
$$




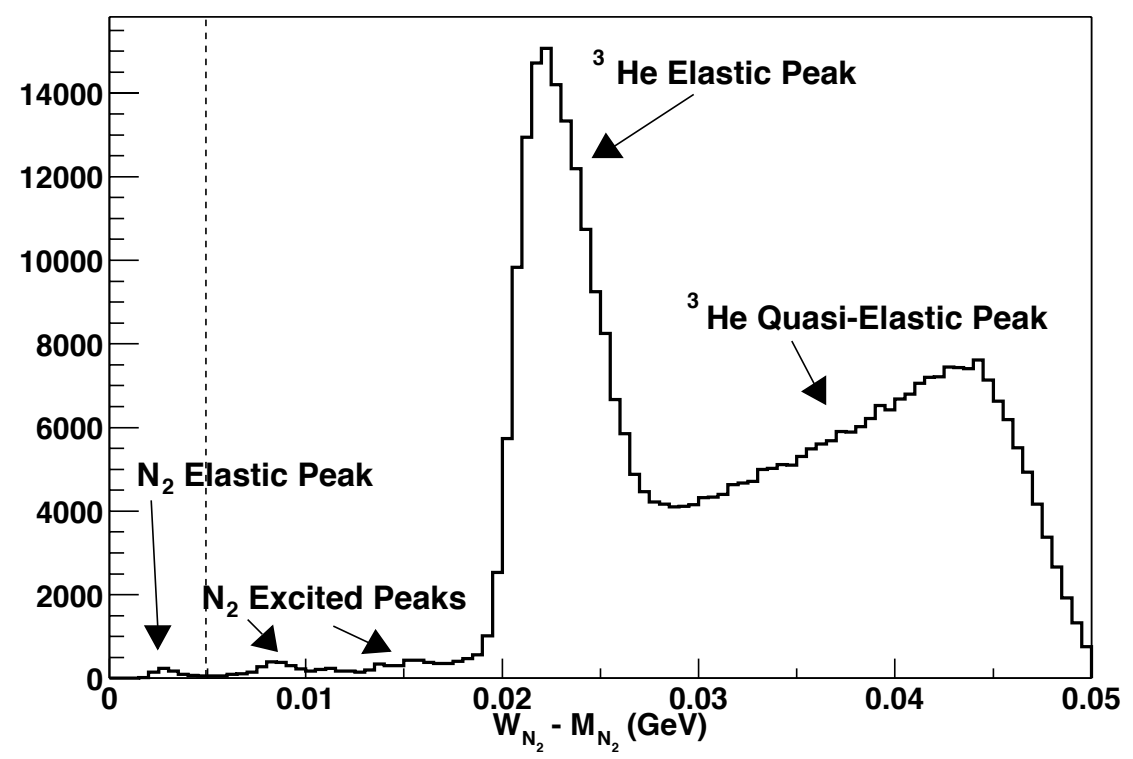

Figure 8.14: A plot of $W_{\mathrm{N}_{2}}-M_{\mathrm{N}_{2}}$ from scattering from a polarized ${ }^{3} \mathrm{He}$ cell.

where the yield, $Y$, can be calculated:

$$
Y=\frac{N T_{\mathrm{tot}}}{T_{\mathrm{acc}} Q}
$$

where $N$ is the number of events in the acceptance cuts, $T_{\text {tot }}$ is the total triggers from scalers, $T_{\text {acc }}$ is the number of triggers in N-tuple and $Q$ is the charge during the run. A cut of $W_{\mathrm{N}_{2}}-M_{\mathrm{N}_{2}}<0.005$ was made to separate the nitrogen elastic peak from the rest of the electron scattering events. $W_{\mathrm{N}_{2}}-M_{\mathrm{N}_{2}}$ can be calculated with kinematic variables:

$$
\begin{aligned}
\left(W_{\mathrm{N}_{2}}-M_{\mathrm{N}_{2}}\right)^{2} & =M_{\mathrm{N}_{2}}^{2}+2 M_{\mathrm{N}_{2}} \nu-Q^{2} \\
\nu & =E-E^{\prime}(1+\delta p / p) \\
Q^{2} & =-\nu^{2}+p_{x}^{2}+p_{y}^{2}+p_{z}^{2} \\
p_{x} & =-E^{\prime}(1+\delta p / p) \sin \theta \\
p_{y} & =-E^{\prime}(1+\delta p / p) \cos \theta \sin \left(\phi_{0}+\phi\right) \\
p_{z} & =E-E^{\prime}(1+\delta p / p) \cos \theta \cos \left(\phi_{0}+\phi\right)
\end{aligned}
$$




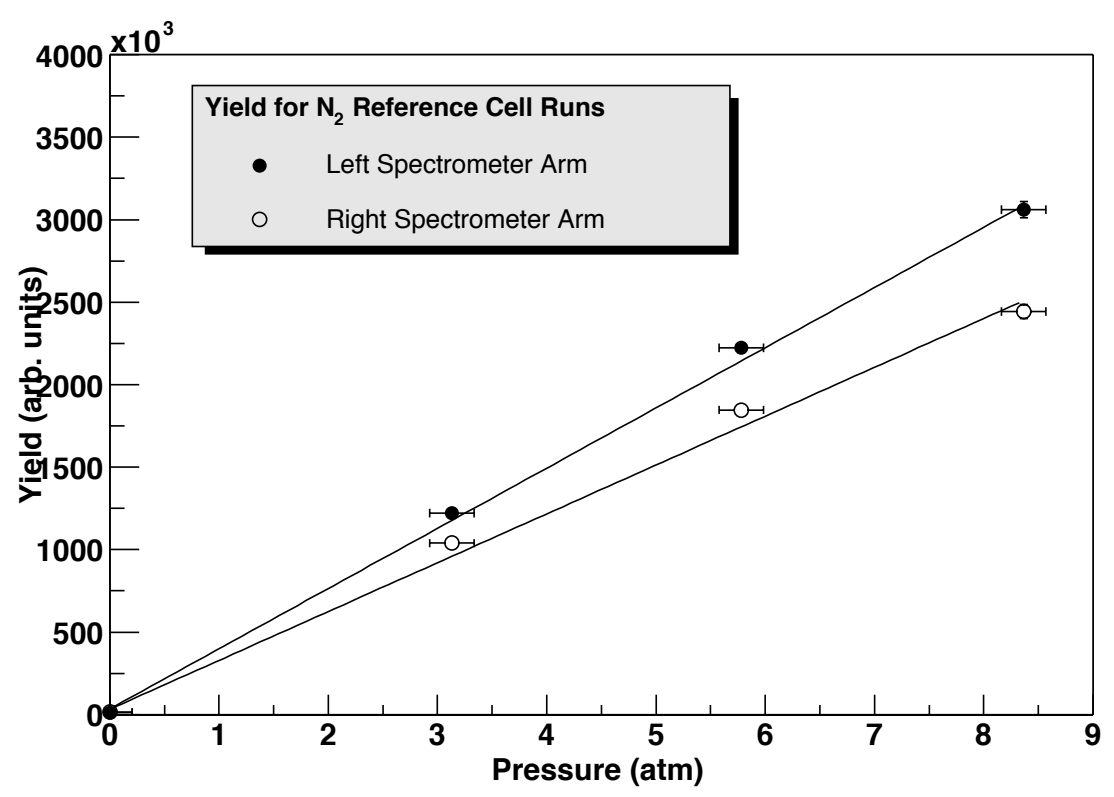

Figure 8.15: The yield from increasing pressures of nitrogen in the reference cell for both the left and right spectrometer arms.

where $M_{\mathrm{N}_{2}}$ is the mass of nitrogen nucleus $(13.047 \mathrm{GeV}), E$ is the beam energy, $E^{\prime}$ is the central scattering energy, $\delta p / p$ is the relative difference in momentum from the central momentum of the spectrometer, $\theta$ is the vertical scattering angle, $\phi_{0}$ is the central horizontal scattering angle of the spectrometer and $\phi$ is the horizontal scattering angle with respect to $\phi_{0}$. It is important that $\phi_{0}$ is positive for the left spectrometer and negative for the right spectrometer. The rest of the acceptance cuts were made tight since statistics was not a problem and end window dilution needed to be kept to a minimum.

A plot of the yield for the nitrogen reference cell in the left and right arm is shown in Fig. 8.15. The pressures associated with each run have significant uncertainty since the reference cell leaked. The pressures for the reference cell were measured before and after the run and the leaking was assumed to be linear. The runs were short so the leakage wasn't large $(\approx 10 \%)$ during each run and the beam current was steady. 


\begin{tabular}{lcc}
\hline \hline Source & $n_{0}$ (Amagats) & Uncertainty (Amagats) \\
\hline Left Arm Spectrometer & 0.0828 & 0.083 \\
Right Arm Spectrometer & 0.111 & 0.088 \\
Fill Density & 0.0673 & 0.0034 \\
\hline \hline
\end{tabular}

These plots can be used to calculate the pressure from nitrogen in the polarized ${ }^{3} \mathrm{He}$ cell. The fits to these curves are:

$$
\begin{aligned}
p_{\text {left }}= & 3.649 \times 10^{5}\left( \pm 7.9 \times 10^{3}\right) Y_{\text {left }}(\text { atm/arb.units }) \\
& +3.49 \times 10^{5}( \pm 3.5) \mathrm{atm} \\
p_{\text {right }}= & 2.961 \times 10^{5}\left( \pm 6.6 \times 10^{3}\right) Y_{\text {right }}(\text { atm } / \text { arb.units }) \\
& +3.26 \times 10^{4}\left( \pm 2.9 \times 10^{4}\right) \mathrm{atm}
\end{aligned}
$$

where $p$ is the nitrogen pressure from the yield on the left and right arm. Unfortunately, both fits show a significant background with a significant error in it. This would be okay if the nitrogen to be measured had a high pressure, but since it is a low pressure it creates a significant uncertainty in the measurement. The source of this background is unknown and warrants further investigation if this technique is going to be used in the future.

Using this fit and the yield extracted with the exact same cuts from a polarized ${ }^{3}$ He runs, one can calculate the nitrogen number density. The results are shown in Fig. 8.5.1. To translate pressure into number density the reference cell temperature of $30{ }^{\circ} \mathrm{C}$ was used. The numbers extracted from this method agreed well with the number from the nitrogen density calculated from the time of the cell filling. This fill density is the same as presented in section 6.6 .8 only for nitrogen. Because the fill density number is so much more accurate than the result from this technique, it is used for the nitrogen dilution for the deep inelastic scattering data. 


\begin{tabular}{ccccc}
\hline \hline Kinematic $\left(\mathrm{GeV}^{2}\right)$ & Left $f$ & Right $f$ & Avg $f$ & Uncertainty \\
\hline Elastic & 0.985 & 0.985 & 0.985 & 0.005 \\
0.565 & 0.952 & 0.951 & 0.952 & 0.005 \\
0.768 & 0.956 & 0.959 & 0.958 & 0.005 \\
0.930 & 0.957 & 0.958 & 0.958 & 0.005 \\
1.107 & 0.965 & 0.957 & 0.961 & 0.005 \\
1.317 & 0.908 & 0.928 & 0.918 & 0.010 \\
\hline \hline
\end{tabular}

Table 8.3: Dilution factors for the 5 kinematic points and the elastic scattering kinematic. Left and Right refer to the Left and Right Spectrometer Arms.

\subsubsection{Dilution Factor from Fill Density}

The dilution factor for all kinematics is calculated with:

$$
f=\frac{Y_{{ }^{3} \mathrm{He}}}{Y_{{ }_{3} \mathrm{He}}+Y_{\mathrm{N}_{2}}}=1-\frac{Y_{\mathrm{N}_{2}}^{\text {ref. }}}{\left(Y_{3} \mathrm{He}+Y_{\mathrm{N}_{2}}\right)} \frac{n_{\text {pol. }}}{n_{\text {ref. }}}
$$

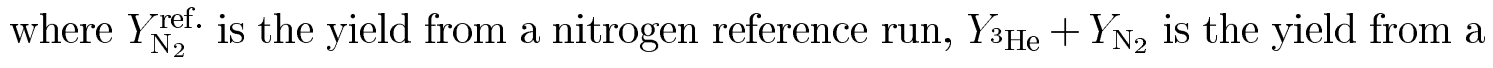
polarized ${ }^{3} \mathrm{He}$ cell with nitrogen in it, $n_{\text {pol. }}$ is the nitrogen density in the polarized ${ }^{3} \mathrm{He}$ cell and $n_{\text {ref. }}$ is the density of the nitrogen in the reference cell when the yield was extracted from it. The yields on the reference cell are extracted with the same cuts and from the polarized ${ }^{3} \mathrm{He}$ cell. The results of these calculation are presented in Table 8.3.

The dilution factor for the $1.317 \mathrm{GeV}^{2}$ kinematic is significantly lower because Virginia One has twice as much nitrogen (0.102 Amagats) in it than Shapiro (0.0552 Amagats).

\subsection{Elastic Asymmetry Check}

To check that everything in the asymmetry analysis is working correctly, a measurement of a known asymmetry is performed and compared to a simulation. During the commissioning period of the experiment, the polarized elastic asymmetry was measured at $E=1.197 \mathrm{GeV}, \phi_{0}=18.6^{\circ}$ and $E^{\prime}=1.1950 \mathrm{GeV}$.

The analyzed results of this measurement are shown in Fig. 8.16. The measured asymmetries were corrected for target polarization, dilution factor and beam 

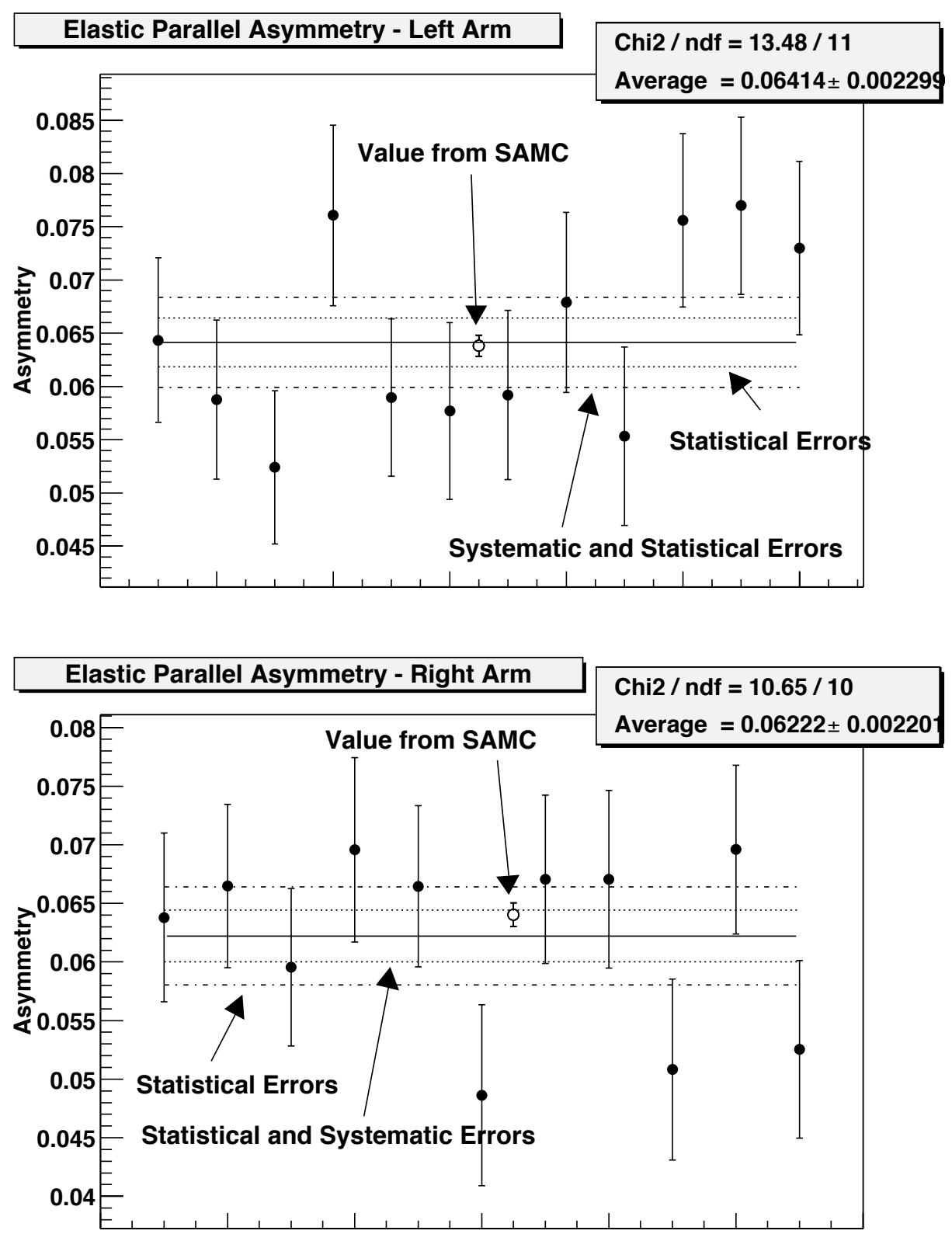

Figure 8.16: The elastic measured in both spectrometers and compared to the simulated value from $S A M C$. 
polarization. The combined systematic and statistical error on the measurement was $6.7 \%$.

The results of these measurements were compared to a value of SAMC (SingleArm Monte Carlo), a simulation program written by Alexandre Deur specifically designed for simulating elastic scattering of the polarized ${ }^{3} \mathrm{He}$ target in Hall A [83]. It takes beam parameters, cell information and spectrometer settings and simulates the experimental data including radiative corrections. More about this simulation can be found in [83],[75] and [41].

There is very good agreement between the simulation and the data taking during the commissioning period of E97-103. This increases the confidence in the DIS asymmetries measured in the experiment.

\subsection{Final Corrected Asymmetries}

The charge and dead-time corrected asymmetry measurements and the asymmetries corrected for target and beam polarization and dilution factor are presented in Table 8.4. The asymmetries were extracted using techniques presented in this chapter. 


\begin{tabular}{ccccc}
\hline \hline$Q^{2}\left(\mathrm{GeV}^{2}\right)$ & $A_{\|}^{\text {raw }}$ & Stat. Err. & $A_{\perp}^{\text {raw }}$ & Stat. Err. \\
\hline 0.565 & -0.00466 & 0.00032 & 0.00087 & 0.00011 \\
0.768 & 0.00538 & 0.00044 & -0.00105 & 0.00016 \\
0.930 & 0.00438 & 0.00040 & -0.00105 & 0.00015 \\
1.107 & -0.00478 & 0.00038 & 0.000582 & 0.00015 \\
1.317 & -0.00505 & 0.00046 & 0.000570 & 0.00016 \\
\hline \hline$Q^{2}\left(\mathrm{GeV}^{2}\right)$ & $A_{\|}$ & Stat. Err. & $A_{\perp}$ & Stat. Err. \\
\hline 0.565 & -0.0156 & 0.0013 & 0.00371 & 0.00054 \\
0.768 & -0.0162 & 0.0015 & 0.00352 & 0.00058 \\
0.930 & -0.0148 & 0.0015 & 0.00363 & 0.00059 \\
1.107 & -0.0140 & 0.0013 & 0.00196 & 0.00055 \\
1.317 & -0.0151 & 0.0015 & 0.00182 & 0.00055 \\
\hline
\end{tabular}

Table 8.4: Tables of the physics asymmetries measured in E97-103. The top table has raw asymmertries which have only been corrected for dead-time and charge. The lower table asymmetries have been corrected for target polarization, beam polarization and dilution factor. 


\section{CHAPTER 9}

\section{Radiative Corrections}

\subsection{Introduction to Radiative Corrections}

In fixed-target inclusive electron scattering, the cross-section is measured by counting the rate of electrons that scatter from a target into a certain angle and momentum from an electron beam of known current and energy. The cross-sections computed from the spin-structure functions in chapter 2 assume that the only interaction made by the scattered electron is a one-photon exchange with the target as represented by Fig. 9.1. Due to both experimental conditions and unavoidable consequences of QED, electrons can lose energy both before and after scattering from the target. This results in a measured cross-section different from the one-photon exchange cross-section (or Born cross-section, as it is commonly called). Fortunately, the various processes that produce the energy loss in the electrons are known and can be calculated. Adjustments made from these calculations to extract the Born cross-section are known as radiative corrections.

Radiative corrections are split into two types internal and external. Internal radiative corrections result from inherent complications in electron scattering such

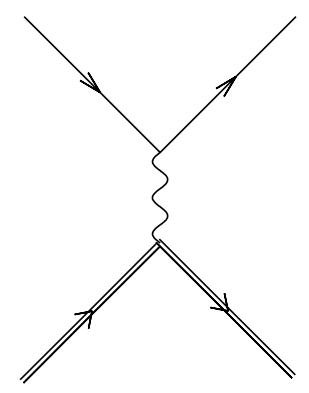

Figure 9.1: Lowest-order electron scattering diagram. 
as vacuum polarization and electron vertex corrections. External corrections are from electron interactions with material in the electron's path. A further contribution to the radiative corrections results from electrons that lose enough energy to undergo elastic and quasi-elastic scattering. Because these types of scattering can be separated from the inelastic cross-section, their corrections are calculated independently.

Since E97-103 is a precision measurement of small asymmetries, accurate calculations of the radiative corrections is critical to reducing the final uncertainties. The world data, in particular the Jefferson Lab E94-010 experiment data set, was used to create the most accurate models possible to do the corrections. Nevertheless, because there are gaps and uncertainty in the world data, as much effort as creating the models must go into estimating the uncertainties resulting from them.

\subsection{Methods of Calculating Radiative Corrections}

\subsubsection{Internal Radiative Corrections}

The Born term is the leading order, and dominant term, in the QED perturbative expansion of the electron scattering cross-section. The next order involves interference terms between the Born scattering diagram and four higher-order diagrams [23]. The four diagrams are vertex corrections, two external leg corrections and the vacuum polarization. The vertex corrections and two external leg corrections contain infrared divergences that can be canceled by including bremsstrahlung diagrams. The calculations of these terms is complex, but well-known. A detailed discussion of these calculations can be found in [23].

The internal radiative corrections in this analysis were calculated using POLRAD 2.0 [84]. This program is based on radiative techniques developed by [85] and [86]. It was chosen because it could handle both unpolarized radiative corrections and polarized radiative corrections. 
However, for this analysis the models for the structure functions in POLRAD 2.0 needed to be updated with the latest data. For the inelastic calculations, this consisted of a straight-forward replacement of the structure functions in the code with improved fits to world data. However, for quasi-elastic scattering, the radiative procedure needed to be modified to incorporate quasi-elastic peaks of a finite width. This modified version of POLRAD 2.0 was written by Seonho Choi [20] for the E94010 analysis and slight modifications were made by myself for this analysis. It was used only for the quasi-elastic and elastic tail calculations.

\subsubsection{External Radiative Corrections}

External radiative corrections result from electron interactions with matter, before and after scattering from the target. The majority of the external radiative correction is the energy loss from bremsstrahlung (electron straggling), but a small amount is also from energy loss due to ionization.

The method of calculating the external radiative corrections in this analysis was based on the work of Mo and Tsai [87] and developed further by Stein, S. et al [88]. This method uses the peaking approximation which states that while many electrons lose energy to bremsstrahlung before or after scattering from the target, a negligible amount experience bremsstrahlung both before and after scattering from the target. This is true if the radiation length of material in the electron path is small.

The calculation of the external radiative corrections requires the internally radiated cross-section. For unpolarized scattering, an internal radiator can be used to calculate the internal cross-section as described by Mo and Tsai [87]. However, for a polarized cross-section, calculations from POLRAD are necessary.

The materials in the electron path for E97-103 are listed in Table 9.1 [89]. The average radiation length used for the incident electron path is $0.00781 \mathrm{~cm}$. The 


\begin{tabular}{lccc}
\hline \hline \multicolumn{4}{c}{ Before Scattering off Target } \\
\hline Material & Rad. Length $\left(\mathrm{g} / \mathrm{cm}^{2}\right)$ & Density $\left(\mathrm{g} / \mathrm{cm}^{3}\right)$ & Thickness \\
\hline Beryllium & 65.19 & 1.848 & 16 mils $(0.0406 \mathrm{~cm})$ \\
Air & 36.66 & 0.001205 & $5 \mathrm{~cm}$ \\
Aluminum & 24.01 & 2.7 & 15 mils $(0.0381 \mathrm{~cm})$ \\
Cell End Window & 19.5 & 2.76 & $0.0120 \mathrm{~cm}$ \\
${ }^{3}$ He in cell & 67.42 & 0.001374 & $20.0 \mathrm{~cm}$ \\
\hline \multicolumn{4}{c}{ After Scattering off Target } \\
\hline Material & Rad. Length $\left(\mathrm{g} / \mathrm{cm}^{2}\right)$ & Density $\left(\mathrm{g} / \mathrm{cm}^{2}\right)$ & Thickness \\
\hline${ }^{3}$ He in cell & 67.42 & 0.001374 & $0.8675 / \sin \phi \mathrm{cm}$ \\
cell wall & 19.5 & 2.76 & $0.145 / \sin \phi \mathrm{cm}$ \\
${ }^{4}$ He & 94.32 & 0.0001249 & $45.7 \mathrm{~cm}$ \\
Aluminum & 24.01 & 2.7 & 15 mils $(0.0381 \mathrm{~cm})$ \\
Air & 36.66 & 0.001205 & $65.1 \mathrm{~cm}$ \\
Kapton & 40.56 & 1.42 & 14 mil $(0.0356 \mathrm{~cm})$ \\
Titanium & 16.17 & 4.54 & 4 mil $(0.0102 \mathrm{~cm})$ \\
\hline \hline
\end{tabular}

Table 9.1: A list of materials and their radiation lengths and densities in the incident and scattering electron path. $\phi$ is the central angle of the spectrometer.

average radiation length used for the scattering electron path is 0.0750 for $18.6^{\circ}$ and $0.0860 \mathrm{~cm}$ for $15.8^{\circ}$. The error on the incident path radiation length is $\approx 3 \%$. The error on the scattering path radiation length is $\approx 10 \%$. These errors are mostly due to uncertainty in the thickness of the materials in the electron path.

\subsubsection{The Radiative Corrections Procedure}

To use POLRAD to calculate radiative corrections, one gives the beam energy, $x$ and $y=\nu / E$ as input, and POLRAD will calculate the internal radiative corrections to the unpolarized, longitudinally polarized and transversely polarized cross-sections. The external radiative corrections require a significant amount of cross-section information to be calculated; consequently, the internal cross-section needs to be calculated for many kinematics.

This calculation is based on models of the structure functions, which are based on the world data. If the Born cross-section plus the radiative correction does not agree with the experimental data, the model can be adjusted in areas where there is 


\begin{tabular}{lccccc}
\hline \hline Cross-section & $Q^{2}=0.54$ & 0.77 & 0.93 & 1.14 & $1.34 \mathrm{GeV}^{2}$ \\
\hline Internal Unpol. & 1.052 & 0.4129 & 0.2258 & 0.1693 & 0.0966 \\
Total Unpol & 0.8547 & 0.3158 & 0.1791 & 0.1332 & 0.0767 \\
Internal Long. Pol. & -0.0778 & -0.0292 & -0.0187 & -0.01324 & -0.0086 \\
Total Long. Pol. & -0.0632 & -0.0222 & -0.0147 & -0.0100 & -0.0067 \\
Internal Trans. Pol. & -0.0475 & -0.0187 & -0.0135 & -0.0091 & -0.0066 \\
Total Trans. Pol. & -0.0397 & -0.0148 & -0.0109 & -0.0071 & -0.0054 \\
\hline \hline
\end{tabular}

Table 9.2: Elastic tails for the five E97-103 kinematic points. All cross-sections are in $n b /(G e V-S r)$.

uncertainty in the world data until the corrections agree with the experiment. This process usually requires several iterations to get the model and experimental data to agree.

\subsection{Elastic and Quasi-Elastic Radiative Corrections}

\subsubsection{Elastic Radiative Corrections}

The Born elastic cross-section is a delta function at $W^{2}=M^{2}$, but because of radiative corrections can contribute at deep inelastic kinematics. However, in the case of E97-103 the contribution is small.

The ${ }^{3} \mathrm{He}$ elastic radiative corrections are calculated from form factors in [16]. The internal radiation procedure was performed by Seonho Choi's modified edition of POLRAD 2.0. The external radiative corrections were calculated using the peaking approximation method presented in S. Stein, et al [88]. The results are presented in Table 9.2.

The uncertainty of the elastic tail calculations is estimated to be $10 \%$. The major source of uncertainty is the knowledge of the radiation length. Other uncertainties include radiative procedure and knowledge of the ${ }^{3} \mathrm{He}$ form factors. 


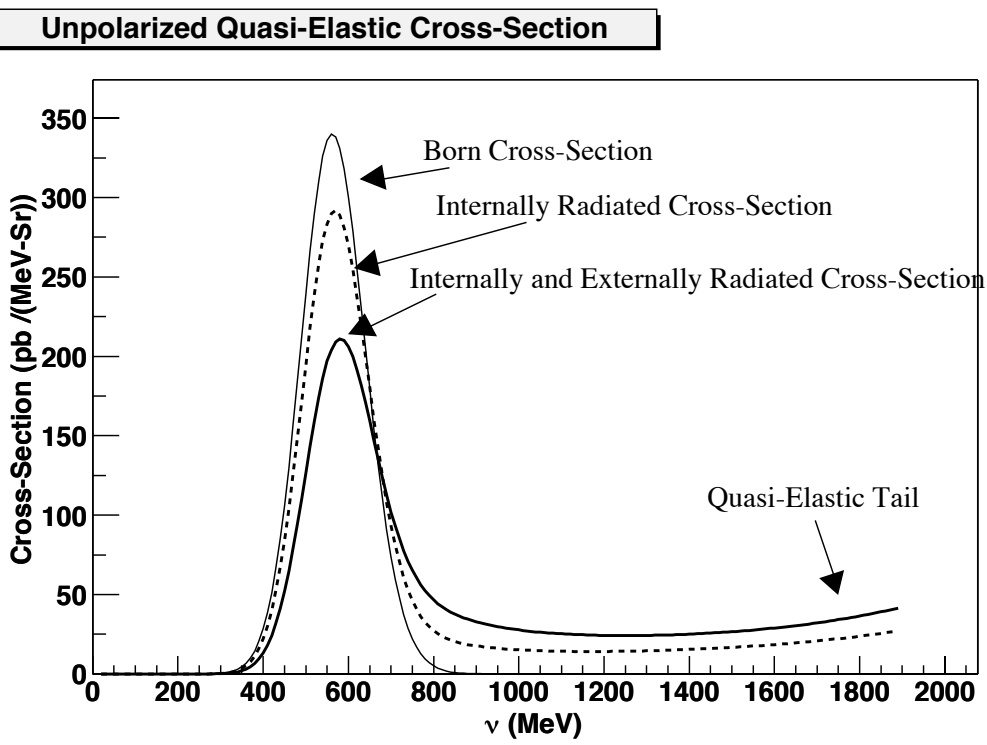

Figure 9.2: A plot of the Born, internally radiated and externally radiative unpolarized quasi-elastic cross-section.

\subsubsection{Unpolarized Quasi-Elastic Cross-section}

The quasi-elastic tail contributes substantially to the cross-section at E97-103 kinematics. To calculate the unpolarized part of the tail, a modified version of the QFS model developed by Lightbody and O'Connell [18][90] was used. The crosssections in the QFS model are derived from nucleons in carbon nuclei and additional modifications are necessary to model the ${ }^{3}$ He nuclei. This has been done by Karl Slifer for the E94-010 analysis [91]. The modifications adjust the $Q^{2}$ dependence of the height of the quasi-elastic peak to fit the quasi-elastic data taken in E94-010. This version of the code is referred to as NQFS. Calculations of the unpolarized quasi-elastic internal and external radiative tail from NQFS are presented in Fig. 9.2 .

The NQFS model for the unpolarized quasi-elastic cross-section was inserted into the modified version of POLRAD 2.0. This was done to increase confidence in the modified code, which has no cross-check for polarized radiative corrections. The calculations of the unpolarized tail from NQFS and the modified version of 
Comparison of Total Radiated Unpol. QE Tail

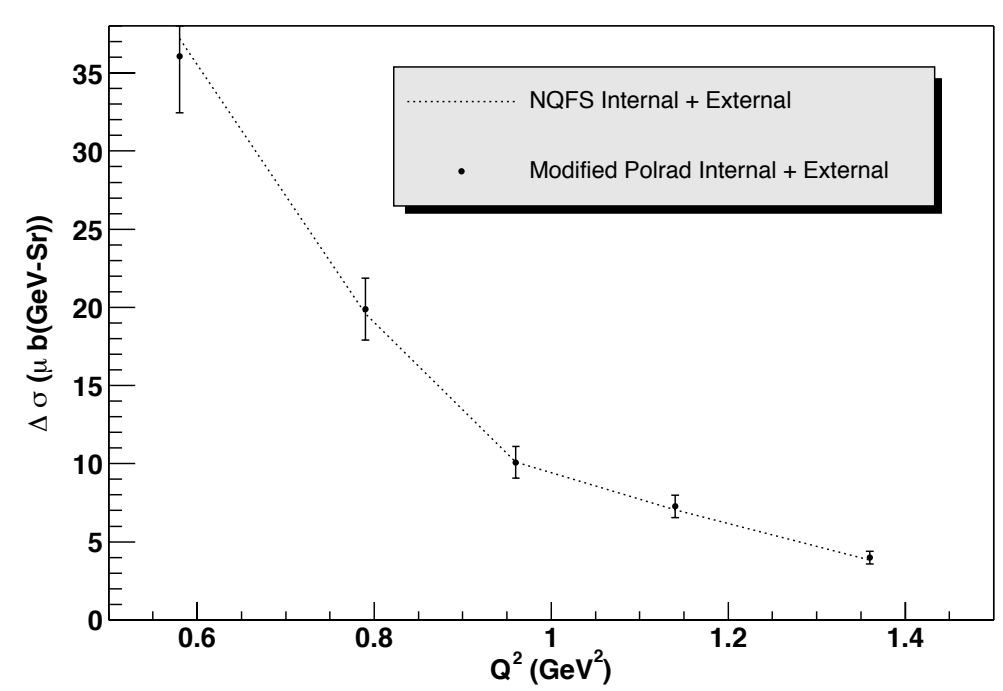

Figure 9.3: Comparison of calculations based on E94-010 data and NQFS Model calculations for the Unpolarized QE Tail.

\begin{tabular}{lccccc}
\hline \hline Cross-section & $Q^{2}=0.54$ & 0.77 & 0.93 & 1.14 & $1.34 \mathrm{GeV}^{2}$ \\
\hline Int. Unpol. QE & 25.52 & 14.68 & 7.90 & 5.88 & 3.33 \\
Tot. Unpol. QE & 36.06 & 19.88 & 10.08 & 7.26 & 3.99 \\
\hline \hline
\end{tabular}

Table 9.3: A list of the unpolarized tails from the quasi-elastic cross-section. Both the internal and total (internal + external) contributions are listed. All cross-sections are in units of $n b /(G e V-S r)$.

POLRAD are shown in Fig. 9.3. The total corrections for both methods agree to the $3 \%$ level. This also builds confidence in that the procedure for calculating external radiative corrections, which is used for elastic, quasi-elastic and inelastic corrections, is correct.

The results from these calculations are listed in Table 9.3. The uncertainty in these calculations is $10 \%$ due to uncertainties in the external radiatve corrections, unpolarized quasi-elastic cross-section and radiative procedure.

\subsubsection{Polarized Quasi-Elastic Radiative Corrections}

The polarized quasi-elastic cross-sections were calculated based on a model of the E94-010 data for $g_{1}^{3} \mathrm{He}$ and $g_{2}^{3} \mathrm{He}$. This model is a fit of a Gaussian distribution 


\section{Integral of Transverse Cross-Section Difference}

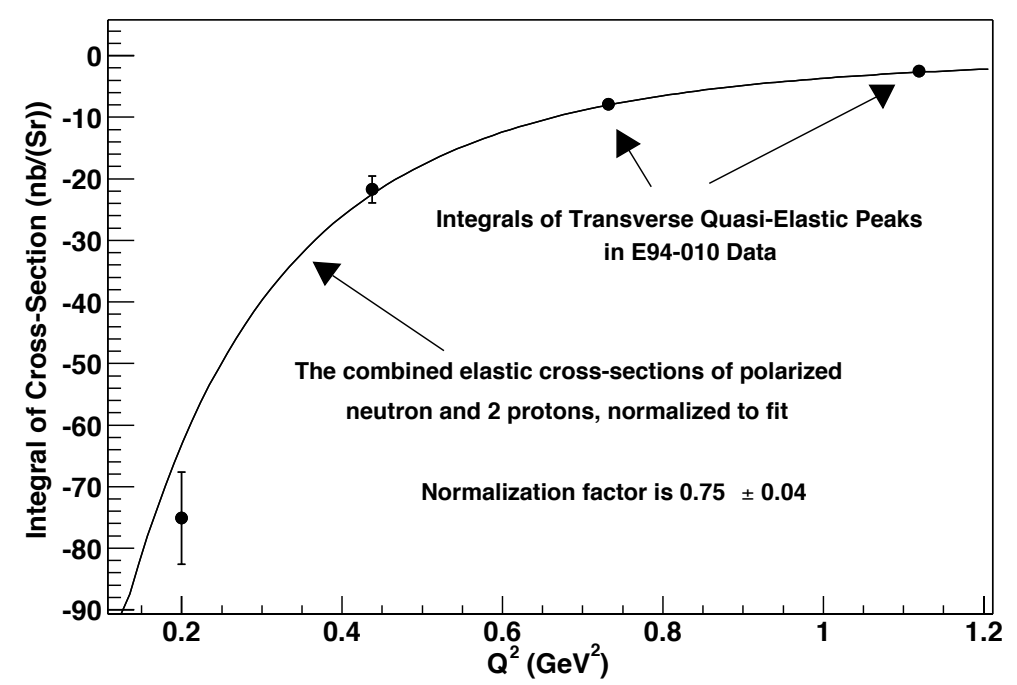

Figure 9.4: Comparison of transverse polarized cross-section from E94-010 and model of the polarized quasi-elastic cross-section.

to the quasi-elastic peak in both the longitudinal and tranverse cross-section differences, calculated from $g_{1}^{3} \mathrm{He}$ and $g_{2}^{3} \mathrm{He}$ for four values of $Q^{2}$. These integrals of the Gaussian in $\nu$ vs. $Q^{2}$ is fit using:

$$
I\left(Q^{2}\right)=-A\left(P_{n} \Delta \sigma_{n}^{\text {elas }}\left(Q^{2}\right)+2 P_{p} \Delta \sigma_{p}^{\text {elas }}\left(Q^{2}\right)\right)
$$

where $A$ is a normalization factor, $P_{n}$ is the polarization of the neutron in polarized ${ }^{3} \mathrm{He}, \Delta \sigma_{n}^{\text {elas }}$ is the polarized part of the elastic cross-section for the neutron, $P_{p}$ is the proton polarization in ${ }^{3} \mathrm{He}$ and $\Delta \sigma_{p}^{\text {elas }}$ is the polarized part of the elastic cross-section for the proton.

For the fit of the transverse cross-section $A=0.745 \pm 0.037$ as shown in Fig. 9.4. This is suprising since it is expected to be near 1.0. There are a few effects that could decrease the quasi-elastic cross-section from the polarized elastic such as final state interactions and meson-exchange effects, but they are expected to be a less than $10 \%$ effect. There is uncertainty in the fit to the E94-010 data at the 10-15\% level, due to the error bars in the data and the fact that the quasi-elastic peak is not exactly Gaussian [92]. It is possible that these errors could be the source of the 


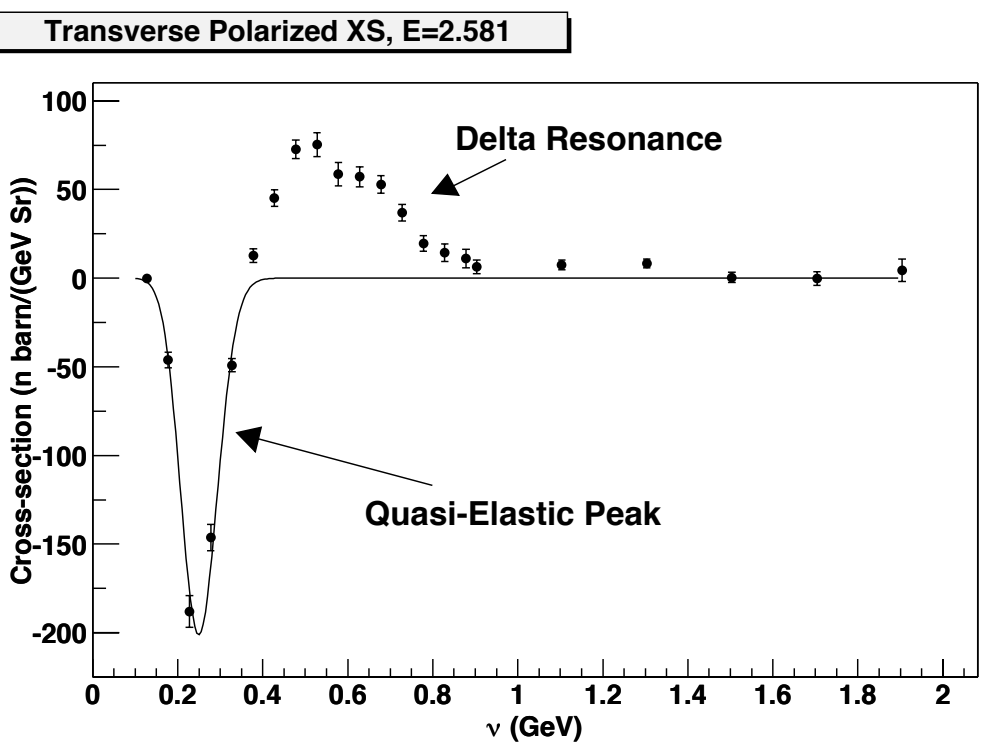

Figure 9.5: Comparison of Transverse Polarized Cross-section from E94-010 and model of the polarized quasi-elastic cross-section.

discrepancy. This analysis uses this normalization factor for both the longitudinal and transverse polarized quasi-elastic tail.

This $Q^{2}$ dependence of the width of quasi-elastic peak in the transversely polarized cross-section is:

$$
w=(0.0185 \pm 0.0027 \mathrm{GeV})+(0.0501 \pm 0.0054 / \mathrm{GeV}) Q^{2}
$$

where $w$ is the width of the Gaussian fit to the transverse quasi-elastic peaks. A study of the quasi-elastic tail at E97-103 kinematics showed that large variations of the width had very small effects on the tail as long as the integral of the quasi-elastic peak was the same. Therefore the uncertainty in the width has little effect on the calculation of the quasi-elastic contribution. A comparison of the model using these fits to the E94-010 data is shown in Fig. 9.5.

Table 9.4 lists the contributions of the polarized quasi-elastic tail to the crosssection at E97-103 kinematics. The error in these tails is estimated to be $20 \%$ based on uncertainty in the model and uncertainty in external radiative corrections. 


\begin{tabular}{lccccc}
\hline \hline Cross-section & $Q^{2}=0.54$ & 0.77 & 0.93 & 1.14 & $1.34 \mathrm{GeV}^{2}$ \\
\hline Int. Trans. QE & -0.551 & -0.309 & -0.186 & -0.128 & -0.082 \\
Tot. Trans. QE & -0.894 & -0.482 & -0.271 & -0.182 & -0.112 \\
Int. Long. QE & 0.186 & 0.102 & 0.071 & 0.049 & 0.036 \\
Tot. Long. QE & 0.247 & 0.131 & 0.084 & 0.056 & 0.040 \\
\hline \hline
\end{tabular}

Table 9.4: Internal and total (internal+external) radiative tails from transverse and longitudingal polarized quasi-elastic scattering. All cross-sections in nb/(GeV-Sr).

\subsubsection{Elastic and Quasi-elastic Cross-section Subtraction}

Once the tails for the elastic and quasi-elastic peaks have been calcuated, their contribution to the physics asymmetry can be subtracted. The resulting asymmetry will be based only on the Born cross-section and radiative corrections from the four inelastic structure functions $F_{1}, F_{2}, g_{1}$ and $g_{2}$. This help simplifies the analysis.

The method of subtracting the elastic and quasi-elastic tails used in this analysis is:

$$
A_{\text {inelas. }}=\frac{A_{\text {meas. }}\left(\sigma_{\text {inelas. }}+\sigma_{\mathrm{qe}}+\sigma_{\text {elas. }}\right)-\Delta \sigma_{\mathrm{qe}}-\Delta \sigma_{\text {elas. }}}{\sigma_{\text {inelas. }}}
$$

where $A_{\text {inelas. }}$ is the asymmetry just from the inelastic part of the cross-section, $A_{\text {meas. }}$ is the measured asymmetry corrected for charge, dead-time, target and beam polarization and nitrogen dilution, $\sigma_{\text {inelas. }}$ is the unpolarized inelastic cross-section, $\sigma_{\mathrm{qe}}$ is the unpolarized quasi-elastic cross-section, $\sigma_{\text {elas. }}$ is the unpolarized elastic cross-section, $\Delta \sigma_{\mathrm{qe}}$ is polarized part of the quasi-elastic cross-section and $\Delta \sigma_{\text {elas. }}$ is the polarized part of the elastic cross-section. This equation applies to both longitudinal and transverse asymmetries.

The tails have been calculated in previous sections, but the unpolarized inelastic cross-sections need to come from our measurement or from the world data. In this analysis, an interpolation of the unpolarized data from E94-010 provides us with cross-sections. This interpolation will be described in the inelastic radiative corrections section.

The longitudinal asymmetries with the quasi-elastic and elastic tails subtracted 


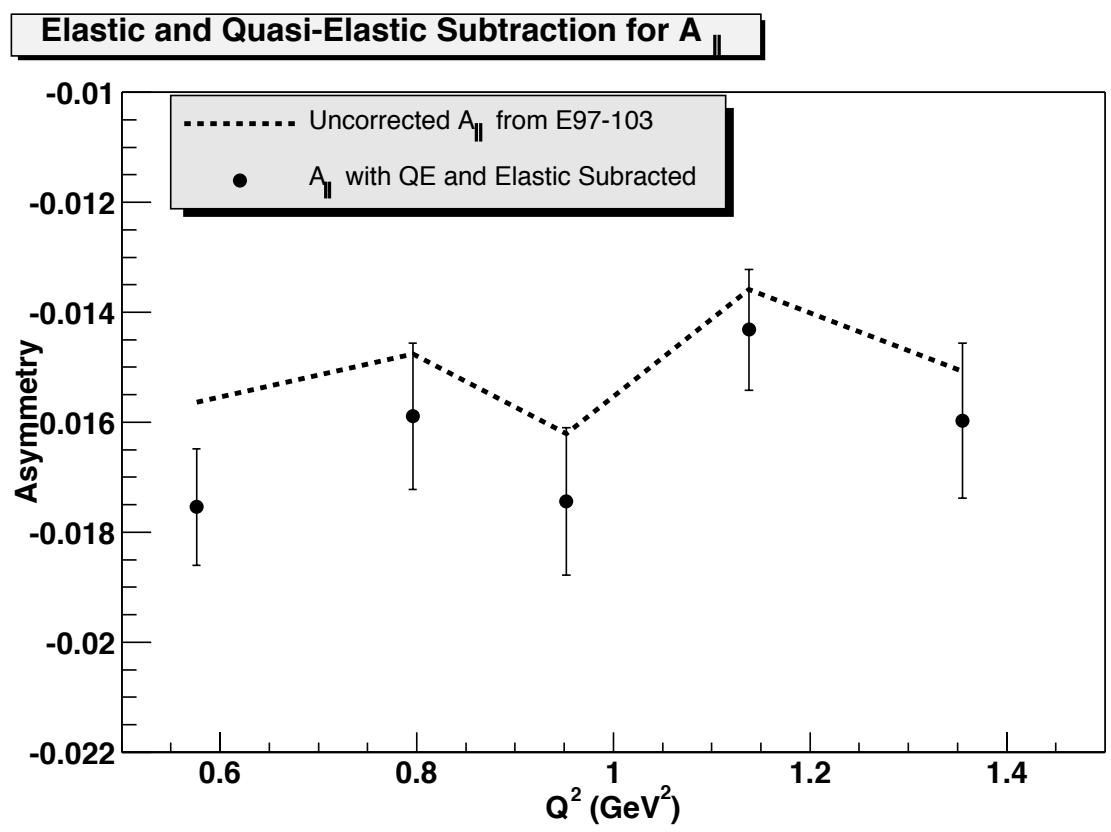

Figure 9.6: The longitudinal asymmetries after the elastic and quasi-elastic tails have been subtracted.

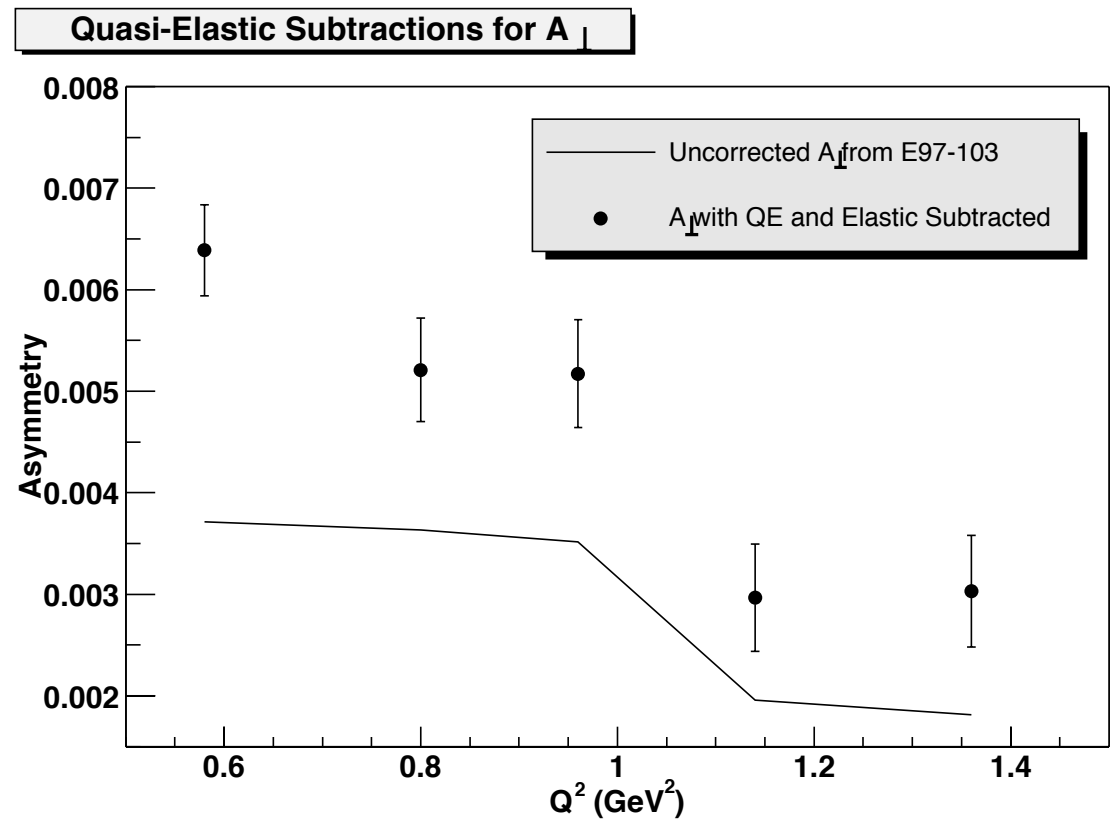

Figure 9.7: The transverse asymmetries after the elastic and quasi-elastic tails have been subtracted. 


\begin{tabular}{ccccc}
\hline \hline Kinematic $\left(\mathrm{GeV}^{2}\right)$ & $A_{\|}^{\text {meas. }}$ & $A_{\|}^{\text {inelas. }}$ & Stat. Err. & Sys. Err. \\
\hline 0.54 & -0.01564 & -0.01754 & 0.0012 & 0.00046 \\
0.77 & -0.01476 & -0.01589 & 0.0016 & 0.00027 \\
0.93 & -0.01621 & -0.01743 & 0.0012 & 0.00031 \\
1.13 & -0.01359 & -0.01432 & 0.0011 & 0.00019 \\
1.34 & -0.01507 & -0.01597 & 0.0014 & 0.00024 \\
\hline Kinematic $\left(\mathrm{GeV}^{2}\right)$ & $A_{\perp}^{\text {meas. }}$ & $A_{\perp}^{\text {inelas. }}$ & Stat. Err. & Sys. Err. \\
\hline 0.54 & 0.003712 & 0.006389 & 0.00050 & 0.00068 \\
0.77 & 0.003632 & 0.005211 & 0.00053 & 0.00040 \\
0.93 & 0.003517 & 0.005173 & 0.00057 & 0.00052 \\
1.13 & 0.001956 & 0.002967 & 0.00053 & 0.00027 \\
1.34 & 0.001816 & 0.003030 & 0.00053 & 0.00032 \\
\hline
\end{tabular}

Table 9.5: The longitudinal and transverse inelastic asymmetries. The statistical error and the systematic error resulting from the subtraction are shown.

are shown in Fig. 9.6 and the subtracted transverse asymmetries are shown in Fig. 9.7. The longitudinal asymmetry is affected less than the transverse asymmetry because the measured asymmetry is larger and the longitudinal polarized crosssection is smaller. Obviously, the quasi-elastic substraction is a large correction to the transverse asymmetry.

The asymmetries before and after subtraction are presented in Table 9.5. The statistical error becomes a smaller percentage of the asymmetry, but significant systematic error is introduced. Most of the the error is due to uncertainty in the unpolarized inelastic cross-section; however, a significant amount enters from uncertainty in the polarized quasi-elastic tail.

\subsection{Inelastic Radiative Corrections}

\subsubsection{Models for Radiative Corrections}

The accuracy of the inelastic radiative corrections depends on the quality of the models for the structure functions. The kinematic region the models need to cover is shown in Fig. 9.8. A resonance region section of this plot was measured experimentally by the Jefferson Lab E94-010 collaboration [62]. This, combined 


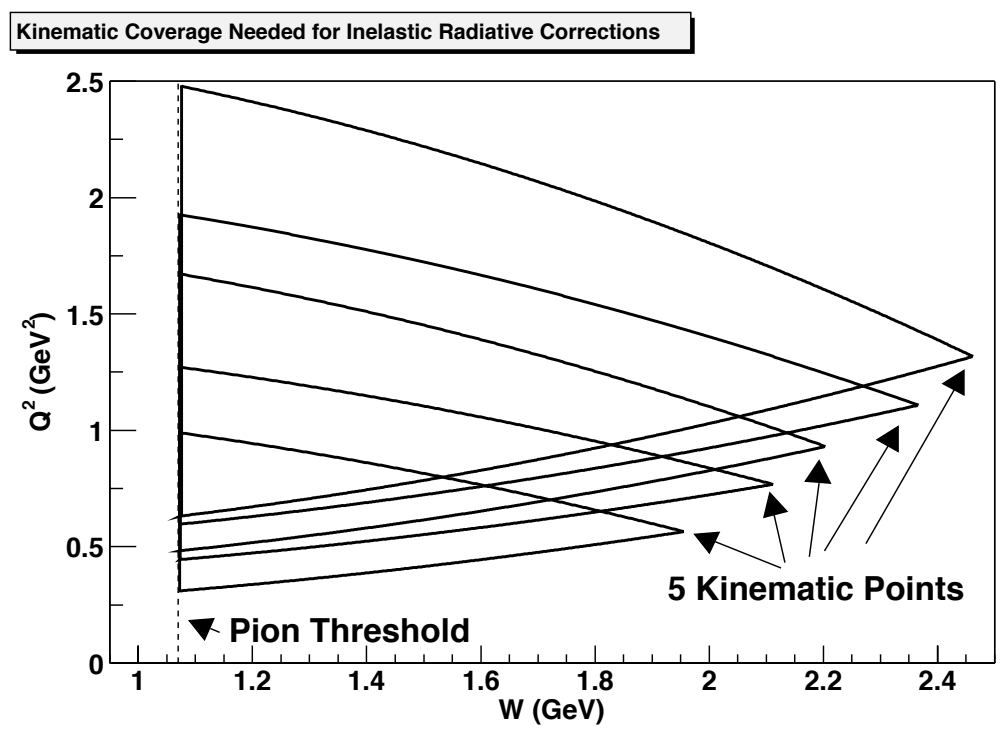

Figure 9.8: The triangles represent the kinematic coverage needed for internal and external radiative corrections for the kinematic point of the right-hand corner of the triangle.

with the significant amount of world data in the DIS region, formed the basis of the models used to calculate radiative corrections. Table 9.6 list all the models used.

While the E94-010 data had been analyzed, no appropriate fit had been made of the data that could be used for the radiative corrections of E97-103. The data set consisted of unpolarized cross-sections, $g_{1}^{3} \mathrm{He}$ and $g_{2}^{3} \mathrm{He}$ for 6 beam energies and a spectrometer angle of $15.5^{\circ}$ [62]. Therefore, considerable effort went into creating a usable interpolation of these data sets. In the case of the unpolarized data, $F_{2}^{3} \mathrm{He}$ was extracted from the unpolarized cross section data by using the world fit to $R$ and then the fit was made to $F_{2}^{3} \mathrm{He}$.

The model of each of the three sets was constructed the same way. The data for the structure function for each beam energy was plotted vs. $W$. Looking at the plot in Fig. 9.9, these fits were made along the slanted lines connecting the dots. A fit was made of each plot with a series of second-order polynomial and Gaussian fits. No effort was made to assume any particular shape in the plots. The emphasis was on creating a smooth fit to the data with no bumps or kinks.

It would be possible to interpolate the data from these fits alone, but two 


\begin{tabular}{|l|c|c|}
\hline \hline Struct. Func. & kinematic region & Source \\
\hline$F_{2}^{3} \mathrm{He}$ & $Q^{2}>1.7 \mathrm{GeV}^{2}$ & $\begin{array}{c}\text { NMC Fits for } F_{2}^{D} \text { and } F_{2}^{p}[93] \\
\text { JLab E94-110 fits for } F_{2}^{p}[94]\end{array}$ \\
\hline$F_{2}^{3} \mathrm{He}$ & $Q^{2}<1.7 \mathrm{GeV}^{2}$ & $\begin{array}{c}\text { E97-103 fit to } \\
\text { of E94-010 unpol. data }\end{array}$ \\
\hline$R$ & All $Q^{2}$ & $\begin{array}{c}\text { JLab Hall C fit [94] } \\
\text { SLAC R1998 fit [32] }\end{array}$ \\
\hline$g_{1}^{3} \mathrm{He}$ & $Q^{2}>1.7 \mathrm{GeV}^{2}$ & $\begin{array}{c}\text { E99-117 fit } g_{1} / F_{1}[41] \\
\text { for proton and neutron }\end{array}$ \\
\hline$g_{1}^{3} \mathrm{He}$ & $Q^{2}<1.7 \mathrm{GeV}^{2}$ & E97-103 fit to E94-010 $g_{1}^{3} \mathrm{He}$ \\
\hline$g_{2}^{{ }^{3} \mathrm{He}}$ & $Q^{2}>1.7 \mathrm{GeV}^{2}$ & $g_{2}^{W W}$ from $g_{1}$ fit above \\
\hline$g_{2}^{{ }_{3} \mathrm{He}}$ & $Q^{2}<1.7 \mathrm{GeV}^{2}$ & E97-103 fit to E94-010 $g_{2}^{3} \mathrm{He}$ \\
\hline \hline
\end{tabular}

Table 9.6: Source of models for structure functions used for the radiative corrections for E97-103.

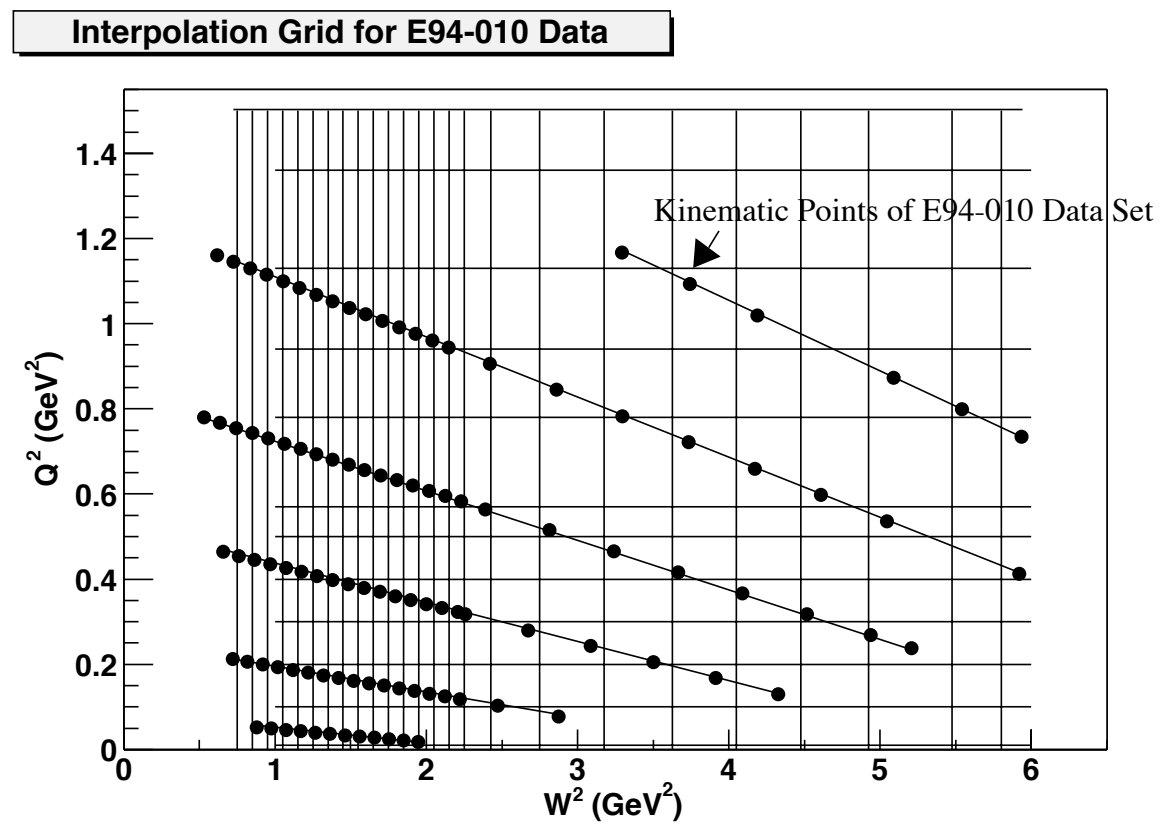

Figure 9.9: The grid used for interpolating the E94-010 data set. 
problems occur. The first is that strong discontinuities can occur away from where the data set lies, particularly in regions at the edge of each data set in $W$. The second is that the E97-103 data need to be put into the model and it does not sit on any one of these lines due to kinematic differences. Therefore a more robust technique is required.

The technique chosen was to use these fits to create data sets at constant $W$, represented by the vertical lines in Fig. 9.9. Each constant $W$ data set had 5-7 points corresponding to 4-6 intersections of the E94-010 data plus a data point at $1.7 \mathrm{GeV}^{2}$ from the DIS fits to ensure a smooth transition between the regions. Fits were made of this data by using a string of 2nd order polynomials and exponential fits. Again an emphasis on smoothness of the fit was made. This was done for 26 values of $W$ for the three structure function data sets.

Again, this is an adequate basis to do interpolation, but the variation between fits at different $W$ 's created many unphysical bumps in the structure functions. Therefore, another fit was done at constant values of $Q^{2}$, which correspond to the horizontal lines in Fig. 9.9. Each fit along constant $Q^{2}$ included 26 points, one from each $W$ fit. Five of the constant $Q^{2}$ values were chosen to correspond to the five data points in E97-103. The fits along $Q^{2}$ were made of Gaussian and polynomial fits to the points. Again an emphasis on smoothness and physical shapes was maintanined.

Once the set of fits at constant $Q^{2}$ were made, a code was written to interpolate between the fits at any $x$ and $Q^{2}$ below $Q^{2}$ of $1.7 \mathrm{GeV}^{2}$. The fits are merged with the higher $Q^{2}$ data by using a set of data at $Q^{2}=1.7 \mathrm{GeV}^{2}$, from the higher $Q^{2}$ fits, in the interpolation. The interpolation is done by a routine based on the polint routine in Numerical Recipes in $C$ [74]. The results of this interpolation can be compared back to the E94-010 data to see the sucess of the interpolation. The unpolarized data from E94-010 is plotted in Fig. 9.10 with the interpolation of the $F_{2}^{3} \mathrm{He}$ data and the world fit for $R$ used to calculate the unpolarized cross-section. 

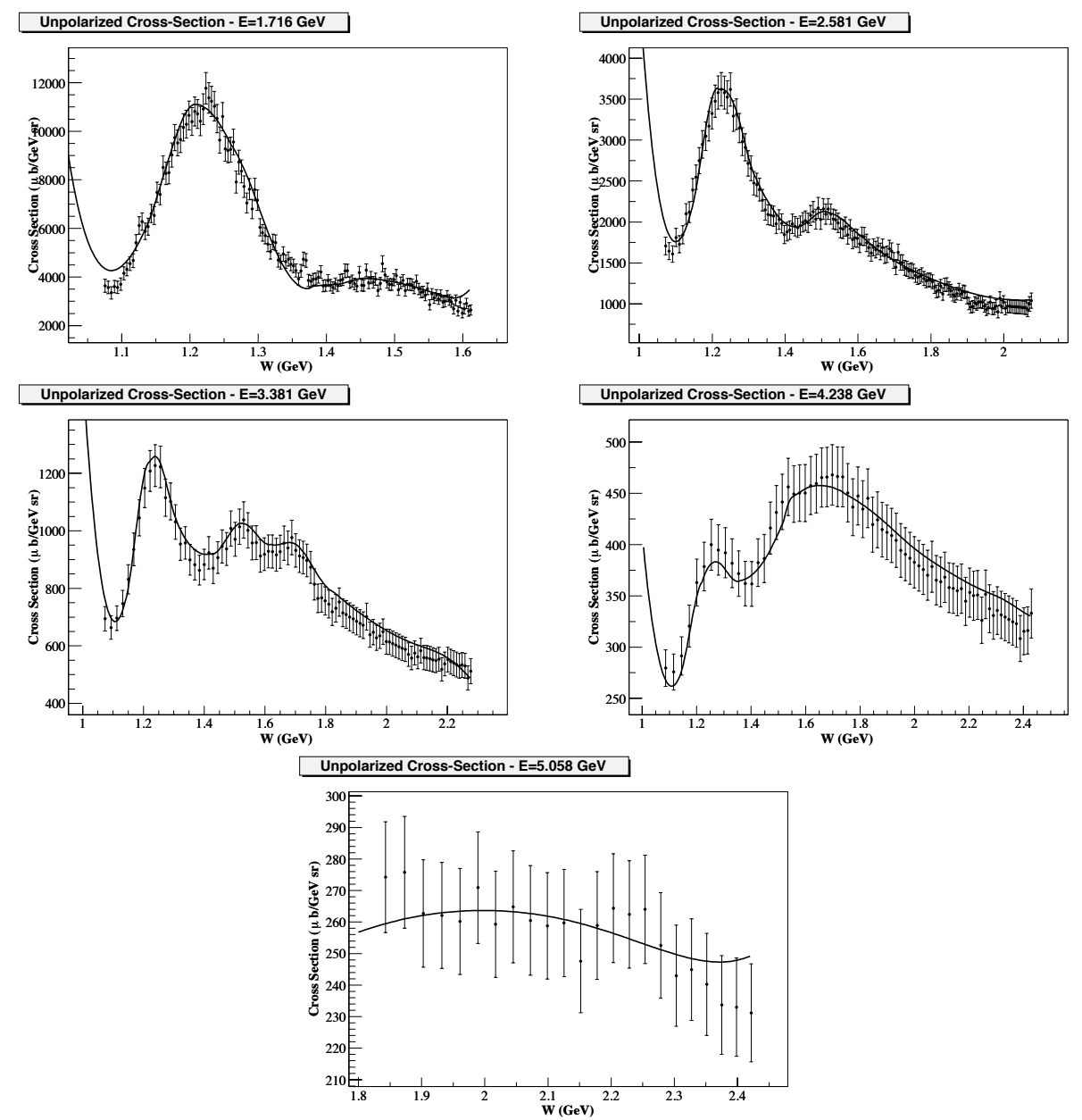

Figure 9.10: Comparison plots between the E94-010 unpolarized cross-section and crosssection calculated from an interpolation of $\mathrm{F}_{2}^{3} \mathrm{He}$.

A comparison of the interpolations of $g_{1}^{3 \mathrm{He}}$ and the E94-010 data is shown in Fig. 9.11. A similar comparison for $g_{2}^{3} \mathrm{He}$ is made in Fig. 9.12.

\subsubsection{Summary of Inelastic Corrections}

POLRAD 2.0 gives the radiative correction for the unpolarized, longitudinally polarized and tranversely polarized cross-section. An example plot of the Born cross-sections and the internally radiative cross-sections for a constant beam energy and spectrometer angles corresponding to the first kinematic is shown in Fig. 9.13. These were calculated for all five kinematics and are presented in Table 9.7. The effect of the radiative corrections on the parallel and perpendicular asymmetries are 

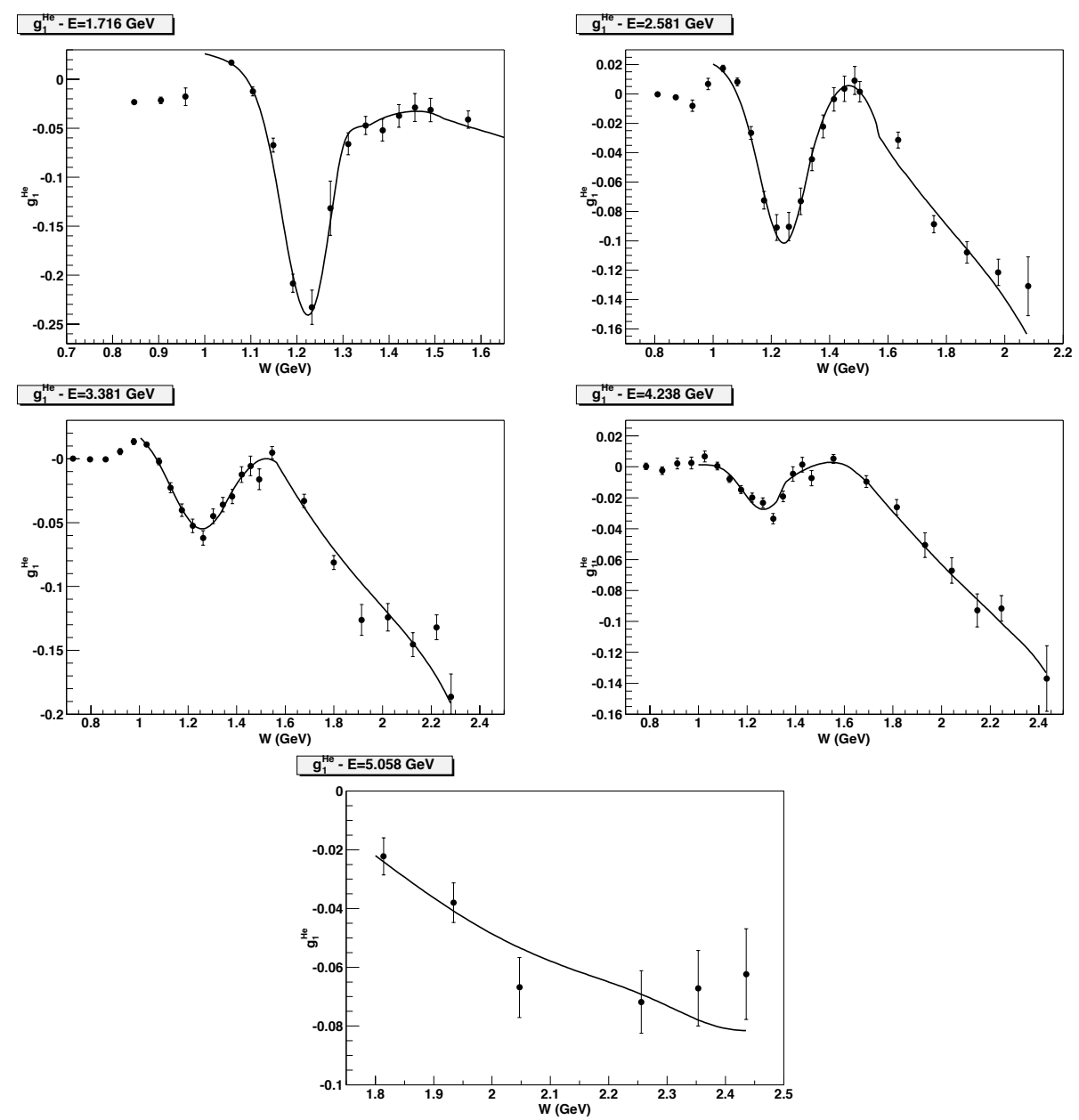

Figure 9.11: Comparison plots between the E94-010 $g_{1}^{3} H e$ and an interpolatiation. Fit stops at pion threshhold. 

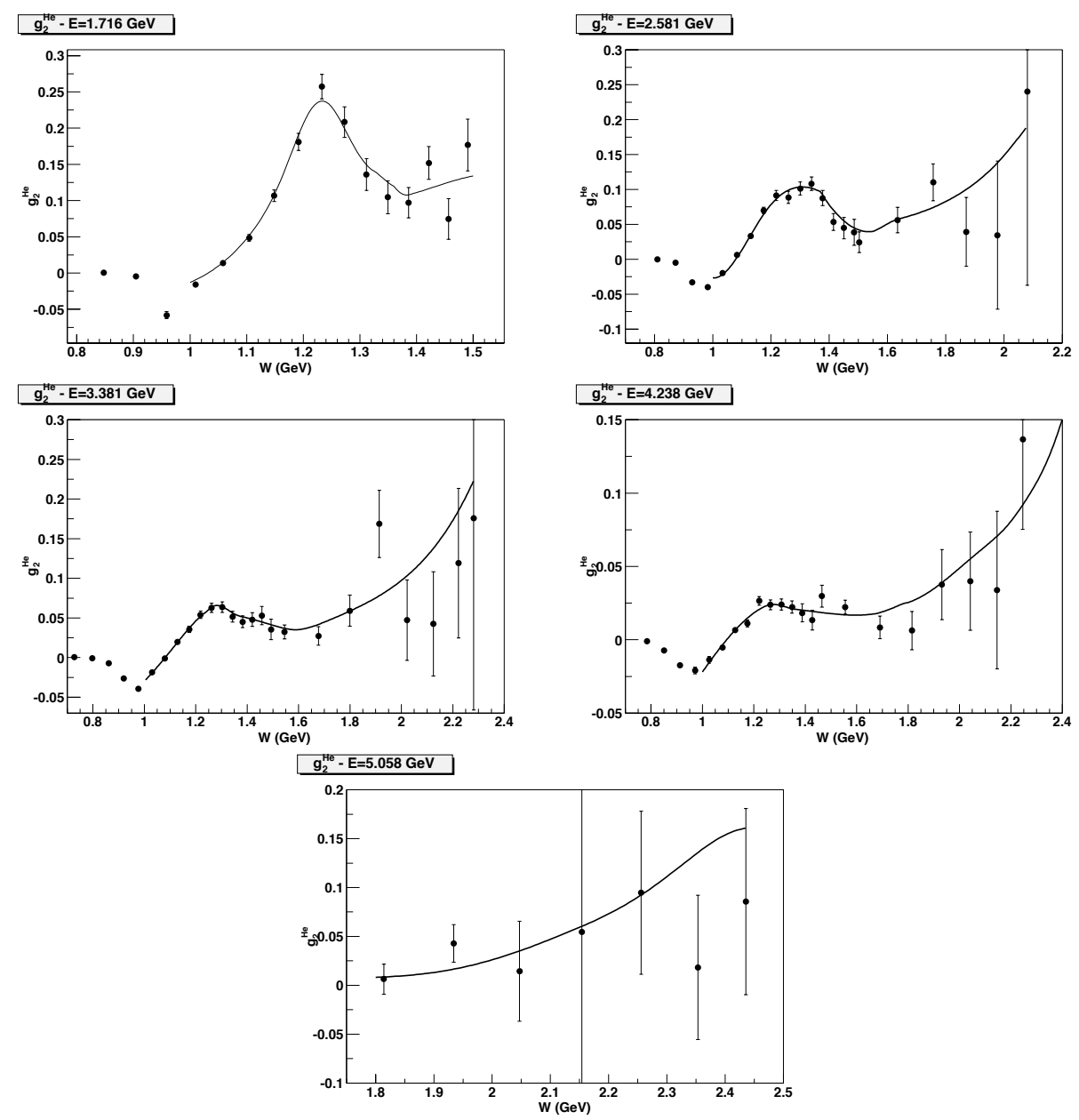

Figure 9.12: Comparison plots between the E94-010 $g_{2}^{3} \mathrm{He}$ and an interpolatiation. Fit stops at pion threshhold. 


\begin{tabular}{lccccc}
\hline \hline Cross-section & $Q^{2}=0.54$ & 0.77 & 0.93 & 1.14 & $1.34 \mathrm{GeV}^{2}$ \\
\hline Unpol Born. & 329.04 & 307.92 & 165.74 & 176.95 & 91.96 \\
Unpol Int. & 376.04 & 348.41 & 185.60 & 197.82 & 102.66 \\
Unpol Tot. & 399.95 & 361.12 & 192.07 & 201.64 & 104.10 \\
\hline Long. Pol. Born & 7.58 & 5.83 & 3.46 & 3.11 & 1.80 \\
Long. Pol. Int & 8.06 & 6.15 & 3.68 & 3.36 & 1.95 \\
Long. Pol. Tot. & 7.69 & 5.68 & 3.54 & 3.25 & 1.89 \\
\hline Trans. Pol. Born & -1.32 & -1.48 & -0.894 & -0.516 & -0.195 \\
Trans. Pol. Int. & -1.96 & -1.80 & -1.05 & -0.65 & -0.27 \\
Trans. Pol. Tot. & -2.40 & -1.85 & -1.00 & -0.62 & -0.29 \\
\hline \hline
\end{tabular}

Table 9.7: A table of the Born cross-sections from the models of the structure functions and the internal and total (internal+external) radiated cross-sections. All cross-sections in $n b /(G e V-S r)$.

shown in Fig. 9.14 and Fig. 9.15.

The uncertainty in the inelastic corrections is determined by varying the model in different ways and seeing what range of values the corrections produce in that cross-section. One has to do this in a way that the radiative corrections produce the correct experimental asymmetryies from the model and can't vary significantly from the world data. From these studies it was found there was at most a $7 \%$ uncertainty in the final cross-section due to uncertainties in the radiative corrections. 

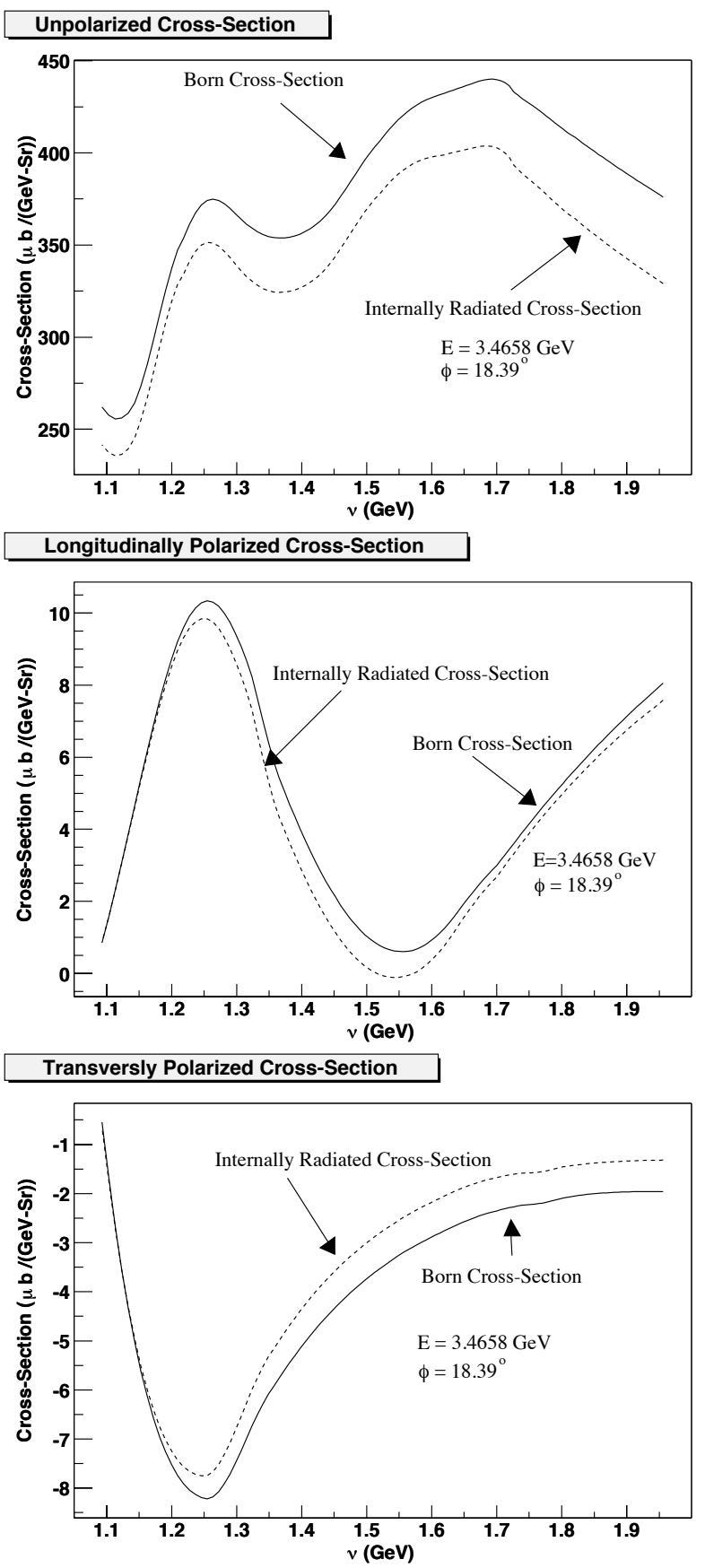

Figure 9.13: Plots of the Born and internally radiated cross-section for the $Q^{2}=0.54 G e V^{2}$ kinematic. 


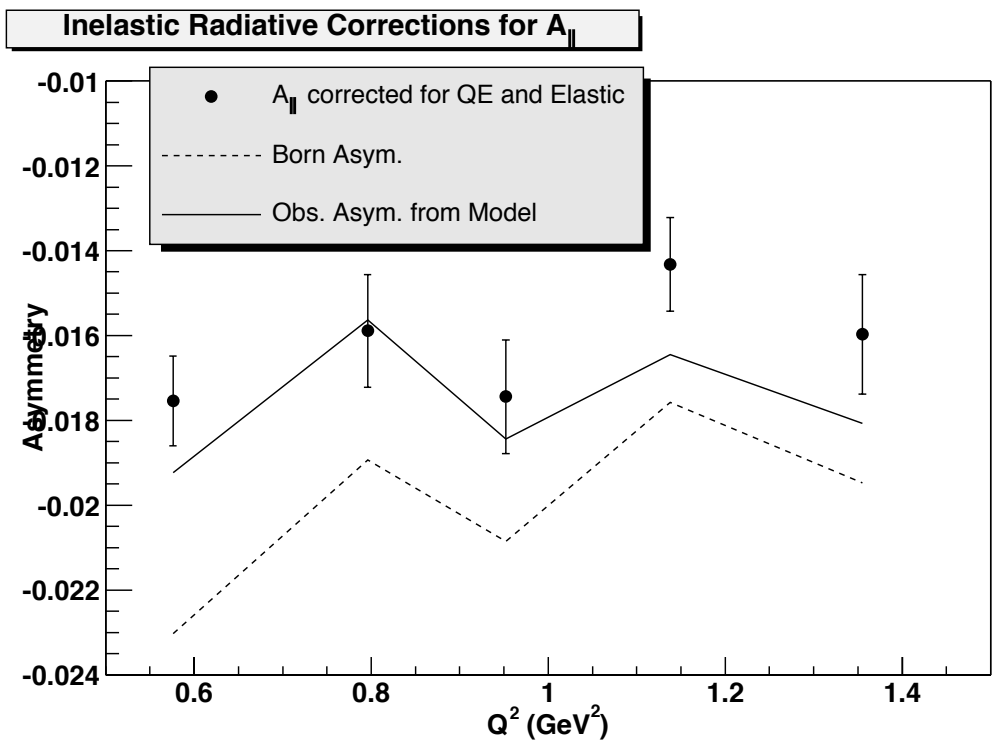

Figure 9.14: The parallel asymmetries compared with calculations from model.

Inelastic Radiative Corrections for $\mathbf{A}_{\perp}$

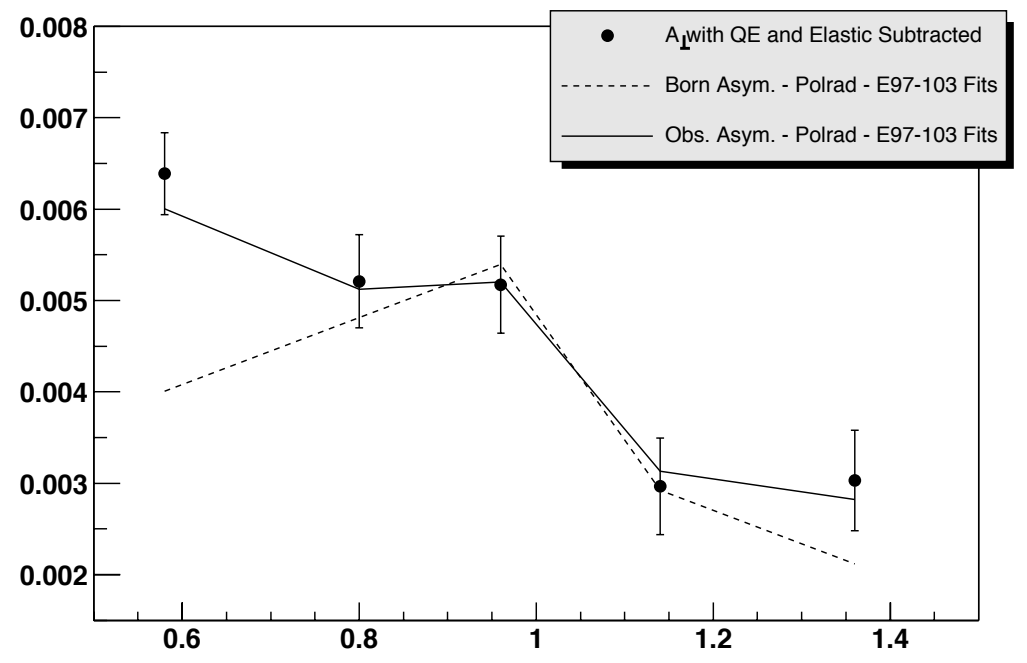

Figure 9.15: The perpendicular asymmety compared with calculations from model. 


\section{CHAPTER 10}

\section{Results and Systematic Errors}

\subsection{Calculating $g_{1}^{3} \mathrm{He}$ and $g_{2}^{3} \mathrm{He}$}

The structure functions $g_{1}^{3} \mathrm{He}\left(x, Q^{2}\right)$ and $g_{2}^{3} \mathrm{He}\left(x, Q^{2}\right)$ can be calculated from the corrected asymmetries using the formula given in Eq. 4.4. As has been previously stated, to extract $g_{1}^{3} \mathrm{He}$ and $g_{2}^{3} \mathrm{He}$ from asymmetries requires knowledge of $F_{1}^{3} \mathrm{He}\left(x, Q^{2}\right)$ and $R\left(x, Q^{2}\right) . R\left(x, Q^{2}\right)$ can be acquired from the Jefferson Lab Hall $\mathrm{C}$ fit [94] used in the radiative corrections. $F_{1}^{3} \mathrm{He}\left(x, Q^{2}\right)$ can be calculated from the interpolation of $F_{2}^{3} \mathrm{He}\left(x, Q^{2}\right)$ created for radiative corrections and $R\left(x, Q^{2}\right)$.

There is a significant disagreement between $F_{2}^{3} \mathrm{He}$ interpolated from the E94-010 data and the calculation of $F_{2}^{3} \mathrm{He}$ from proton and deuterium data. This is shown in Fig. 10.1. It is unclear whether this difference at low $Q^{2}$ is from being on the low edge of the NMC fits for $F_{2}^{D}$ or some problem with the E94-010 interpolation. A large uncertainty of $12 \%$ has been assigned to the structure function for the purposes of this analysis. This could be improved by extracting the unpolarized cross-section from our measured data at each kinematic point. This work is in progress for future.

\subsection{Extracting Neutron Structure Functions from ${ }^{3} \mathrm{He}$}

Since E97-103 measured the structure functions at $x \approx 0.2$ the procedure for going from ${ }^{3} \mathrm{He}$ spin-structure functions to neutron spin-structure functions is straightforward. The equations needed are given by Bissey et al [17]:

$$
\begin{aligned}
g_{1}^{n} & =\frac{1}{P_{n}+0.056}\left[g_{1}{ }^{3} \mathrm{He}+\left(0.014-2 P_{p}\right) g_{1}^{p}\right] \\
g_{2}^{n} & =\frac{1}{P_{n}+0.056}\left[g_{2}{ }^{3} \mathrm{He}+\left(0.014-2 P_{p}\right) g_{2}^{p}\right]
\end{aligned}
$$




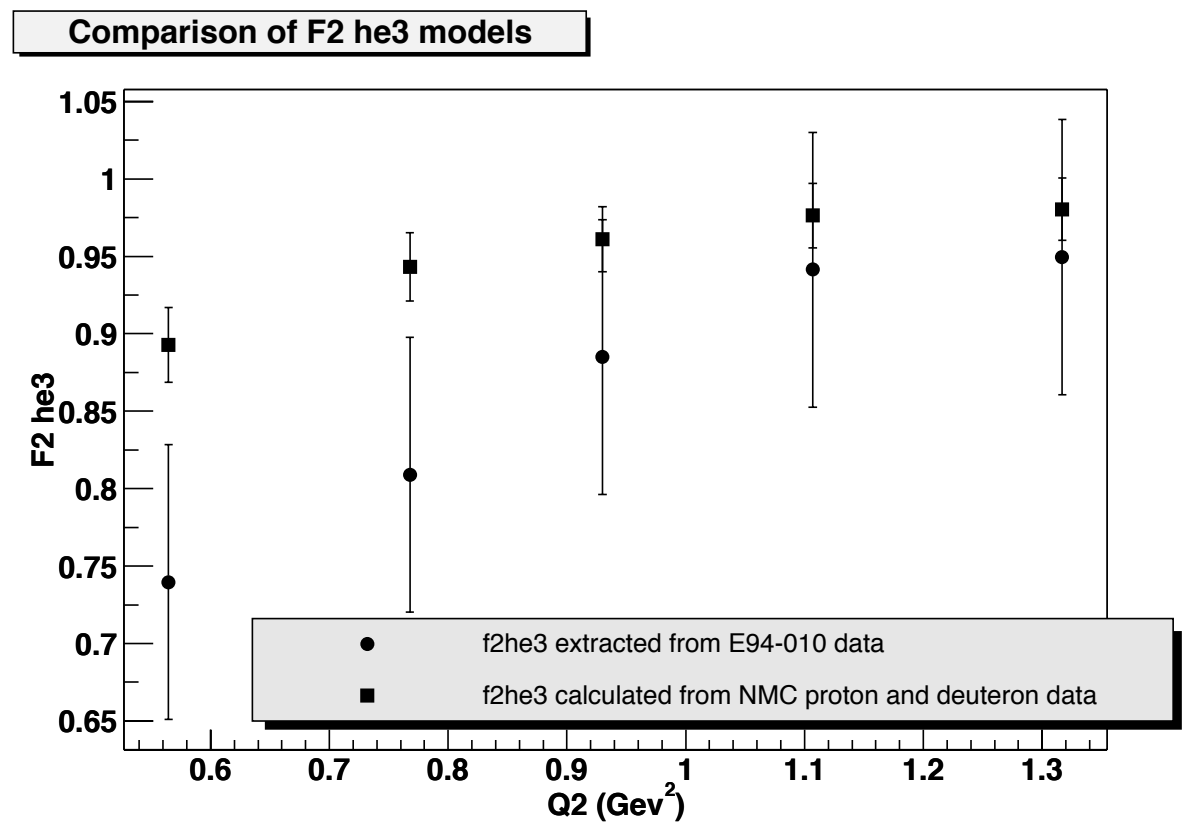

Figure 10.1: A comparison of $F_{2}^{3} \mathrm{He}$ interpolated from E94-010 data and calculated from proton and deuterium data from world fits.

where $P_{n}$ and $P_{p}$ are the effective neutron and proton polarization in ${ }^{3} \mathrm{He} . g_{1}^{p}$ is calculated from a fit in the E99-117 analysis [41]. $g_{2}^{p}$ is calculated from $g_{2}^{p \mathrm{WW}}$. The calculation of $g_{2}^{p}$ WW was done by Wolfgang Korsch [95] and is based on the parton distribution evolution of Blümlein and Böttcher [96].

The neutron and proton polarization in ${ }^{3} \mathrm{He}$ has been the subject of considerable study that is summarized Bissey et al [17]. An estimate of the average and uncertainty in the different calculations for $P_{n}$ and $P_{p}$ has been done by Xiaochao Zheng in her thesis [41] and the results are:

$$
P_{n}=0.86_{-0.02}^{+0.036}, P_{p}=-0.028_{-0.004}^{+0.0094} .
$$

\section{$\underline{10.3 \text { Calculating } g_{2}^{\mathrm{WW}}}$}

Higher twist effects cannot be identified in $g_{2}^{n}$ unless there is an accurate calculation of $g_{2}^{\mathrm{WW}}$. The method used for calculating $g_{2}^{\mathrm{WW}}$ in E97-103 was to use the parton distributions of Blülein and Böttcher [96]. These parton distributions match 
the higher $Q^{2}$ data for $g_{1}^{n}$ and can be evolved down to E97-103 kinematics using DGLAP evolution [96]. This calculation of $g_{2}^{n} \mathrm{WW}$ and its uncertainty for E97-103 kinematics was done by Wolfgang Korsch [95]. There is no way to calculate $g_{2}^{n}$ ww from $g_{1}^{n}$ data at the $Q^{2}$ of E97-103 since the high $x$ data would pass through the resonance region at low $Q^{2}$, where non-DIS effects are significant.

These evolutions of the parton distributions are next-to-leading order in perturbative QCD. There is a large difference between the leading order (LO) calculation and the next-to-leading order (NLO) calculation for $g_{2}^{n} \mathrm{Ww}$. It is possible that there could be another shift in the value of $g_{2}^{n}$ ww if a next-to-next-to-leading order calculation is done [95].

\section{$10.4 \quad$ Results for $g_{1}^{n}$ and $g_{2}^{n}$}

The results of this analysis are presented in Fig. 10.2 and Fig. 10.3. A comparison the data from the SLAC E155X and Jefferson Lab E99-117 experiments are shown in Fig. 10.4. Table 10.1 lists the values for $g_{1}^{n}$ and $g_{2}^{n}$. The plot for $g_{2}^{n}$ along $Q^{2}$ is accompanied by two calculations of $g_{2}^{\mathrm{WW}}$ by Blülein and Böttcher. The two curves presented in the $g_{1}^{n}$ plot are evolved down to the E97-103 kinematics. The comparison to the E155X data has two calculations of $g_{2}^{n}$ Ww , one at $Q^{2}=1.0 \mathrm{GeV}^{2}$ and one at $Q^{2}=5.0 \mathrm{GeV}^{2}$.

Looking at the plot of $g_{2}^{n}$ vs. $Q^{2}$, significant deviation from $g_{2}^{n} \mathrm{WW}$ is seen at the lower $Q^{2}$ points. The systematic errors are significant, but the data is still 1-2 $\sigma$ away from $g_{2}^{n \mathrm{WW}}$. Clearly, reducing the systematic errors will improve the interpretation of the experiment.

The $g_{1}^{n}$ results show good agreement between the Blülein and Böttcher calculations for $g_{1}^{n}$ and the data. Because $g_{1}$ should only contain higher twist terms at $\tau=4$ and higher, a lack of higher twists seen in $g_{1}^{n}$ means that the deviation seen in $g_{2}^{n}$ is likely to be dominated by solely by twist- 3 effects rather than twist- 4 . 


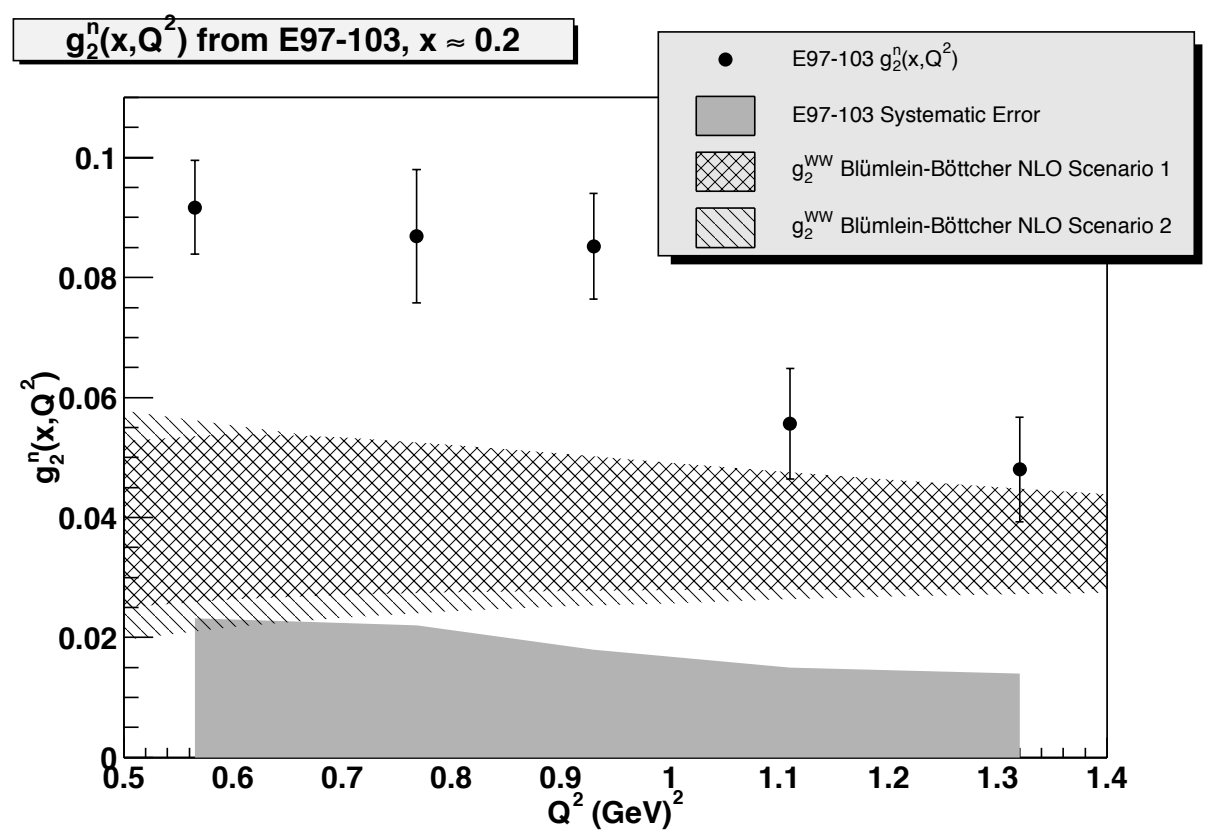

Figure 10.2: The results for $g_{2}^{n}$ and two calculations for $g_{2}^{n} \mathrm{WW}$ from Blülein and Böttcher.

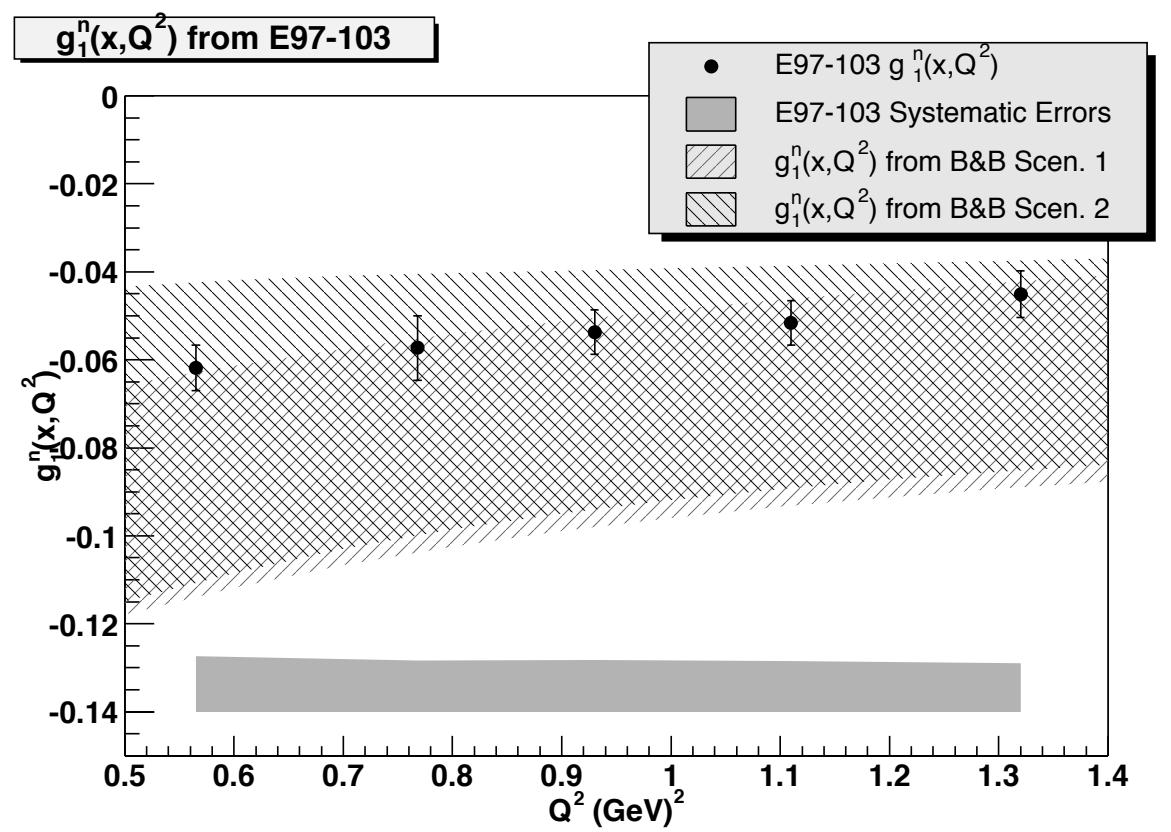

Figure 10.3: The results for $g_{1}^{n}$ and two calculations for $g_{1}^{n}$ from the Blülein-Böttcher parton distributions. 


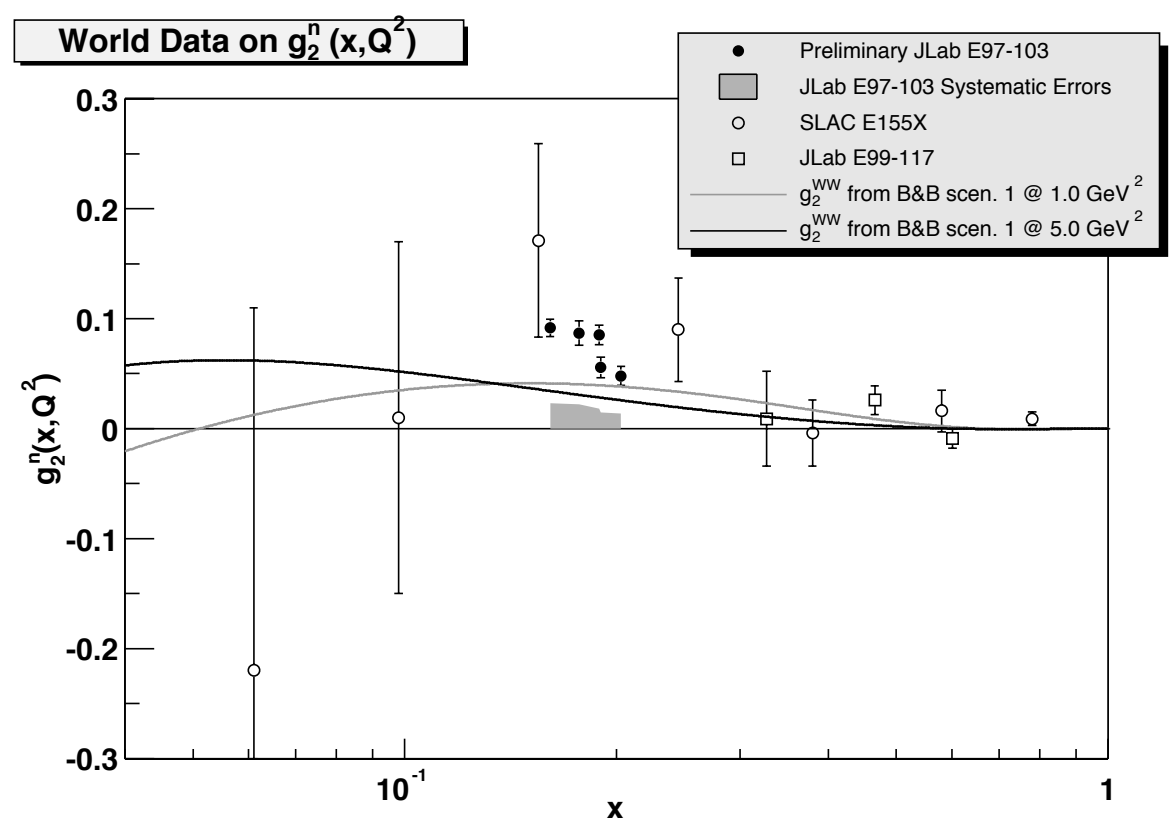

Figure 10.4: The results for $g_{2}^{n}$ vs. $x$ plotted with E155X data.

\begin{tabular}{lccccc}
\hline \hline Quantity & $Q^{2}=0.54$ & 0.77 & 0.93 & 1.14 & $1.34 \mathrm{GeV}^{2}$ \\
\hline$g_{2}^{n}$ & 0.0917 & 0.0869 & 0.0852 & 0.0556 & 0.0480 \\
Stat. Err. & 0.0078 & 0.0111 & 0.0088 & 0.0092 & 0.0087 \\
Sys. Err. & 0.0232 & 0.0220 & 0.0180 & 0.0150 & 0.0140 \\
\hline$g_{1}^{n}$ & -0.0618 & -0.0573 & -0.0537 & -0.0516 & -0.0451 \\
Stat. Err. & 0.0052 & 0.0073 & 0.0051 & 0.0051 & 0.0053 \\
Sys. Err. & 0.0127 & 0.0117 & 0.0118 & 0.0115 & 0.0110 \\
\hline \hline
\end{tabular}

Table 10.1: Values for $g_{2}^{n}$ and $g_{1}^{n}$ and their statistical and systematic error bars. 


\subsection{Summary of Systematic Errors}

Table 10.2 lists the systematic errors associated with every variable that goes into calculating $g_{2}^{n}$. Table 10.3 lists the percentage errors on $g_{2}^{n}$ from each variable by kinematic. Obviously, uncertainty in $F_{2}^{3} \mathrm{He}$ is the largest systematic error. $g_{2}^{n}$ is particularly sensitive to $F_{2}^{3}$ He because it is used in the quasi-elastic and elastic subtraction as well as the calculation of $g_{2}^{3} \mathrm{He}$. Other sources of significant error are the polarized quasi-elastic and inelastic radiative tails and false asymmetries. The statistical error of the data points is on order of these errors.

Significant improvement in the systematic errors can be made by calculating the unpolarized cross-section of ${ }^{3} \mathrm{He}$ using the data of the experiment. Even an analysis of the cross-sections at the 8-10\% level would improve the systematic errors significantly. It is unclear if the error from the false asymmetries or quasi-elastic tail can be improved easily. There will always be significant error from the inelastic tail because there are so many gaps in the structure function data sets.

\section{$\underline{10.6 \text { Conclusion }}$}

E97-103 has measured $g_{2}^{n}$ and found small deviations from $g_{2}^{n}$ WW at the lowest $Q^{2}$ points. Taken as a group, there seems to be clear signs of higher twist effects given the current calculations of $g_{2}^{n} \mathrm{ww}$. However, the systematic error bars are an impediment to making a definitive statement about the interpretation of these deviations.

Significant improvement of the systematic error bars is possible with the data already taken and the analysis available in this dissertation. It is hoped that the E97-103 collaboration will continue its work in extracting this important quantity. 


\begin{tabular}{ccl}
\hline \hline Variable & Error & Description of Variable \\
\hline$A_{\|}$ & $6.8 \%-10.1 \%$ & raw parallel asym. \\
$A_{\perp}$ & $12.4 \%-27.5 \%$ & raw perp. asym. \\
$\delta A$ & $60 \mathrm{ppm}$ & false asymmetries \\
$P_{\text {beam }}$ & $4.0 \%$ & beam polarization \\
$P_{\text {target }}$ & $3.7 \%$ & target polarization \\
$f_{\mathrm{N}_{2}}$ & $0.7 \%$ & dilution factor from $\mathrm{N}_{2}$ \\
$F_{2}^{{ }^{3} \mathrm{He}}$ & $12 \%$ & unpolarized structure function \\
$R$ & $20 \%$ & $\sigma_{L} / \sigma_{T}$ \\
$E$ & $1-3 \mathrm{MeV}$ & incident beam energy \\
$E_{p}$ & $0.01 \%$ & scatter electron momentum \\
$E_{p}$ & $0.1 \mathrm{mrad}$ & central electron scattering angle \\
$\theta$ & $20 \%$ & unpolarized inelastic tail \\
$\sigma_{\mathrm{in}}^{\text {tail }}$ & $15 \%$ & unpolarized quasi-elastic tail \\
$\sigma_{\mathrm{q}}^{\text {tail }}$ & $50 \%$ & long. polarized quasi-elastic tail \\
$\sigma_{\mathrm{l.g}}^{\text {tail }}$ & $25 \%$ & trans. polarized quasi-elastic tail \\
$\sigma_{\mathrm{t.q}}^{\text {tai }}$ & $10 \%$ & unpolarized elastic tail \\
$\sigma_{\mathrm{e}}^{\text {tail }}$ & $10 \%$ & long. polarized elastic tail \\
$\sigma_{\mathrm{l.e}}^{\text {tail }}$ & $10 \%$ & trans. polarized elastic tail \\
$\sigma_{\mathrm{t.e}}^{\text {tail }}$ & $15-30 \%$ & trans. polarized inelastic tail \\
$\sigma_{\text {t.in }}^{\text {tail }}$ & $20 \%$ & long. polarized inelastic tail \\
$\sigma_{\text {t.ii }}^{\text {tail }}$ & 0.04 & neutron polarization in ${ }^{3} H e$ \\
$P_{n}$ & 0.009 & proton polarization in ${ }^{3} H e$ \\
$P_{p}$ & $20 \%$ & spin-structure function \\
$g_{1}^{p}$ & $30 \%$ & spin-structure function \\
$g_{2}^{p}$ & &
\end{tabular}

Table 10.2: Errors on independent variables used to calculate $g_{1}^{n}$ and $g_{2}^{n}$. Percentages are absolute. 


\begin{tabular}{|c|c|c|c|c|c|}
\hline Variable & $Q^{2}=0.56$ & 0.77 & 0.93 & 1.11 & 1.32 \\
\hline$A_{\|}$(stat.) & 1.5 & 2.1 & 1.7 & 2.5 & 3.3 \\
\hline$A_{\perp}$ (stat.) & 8.3 & 13 & 10 & 16 & 18 \\
\hline$\delta A$ & 4.7 & 5.2 & 3.8 & 6.5 & 6.8 \\
\hline$P_{\text {beam }}$ & 2.7 & 3.4 & 2.7 & 2.9 & 3.1 \\
\hline$P_{\text {target }}$ & 2.6 & 3.5 & 2.6 & 2.6 & 2.8 \\
\hline$f_{\mathrm{N}_{2}}$ & 0.6 & 0.8 & 0.6 & 0.7 & 0.7 \\
\hline$F_{2}^{33 \mathrm{He}}$ & 16.6 & 18.0 & 14.7 & 15.6 & 18.0 \\
\hline$R$ & 2.2 & 2.0 & 2.2 & 2.0 & 3.0 \\
\hline$E$ & 0.2 & 0.05 & 0.03 & 0.02 & 0.04 \\
\hline$E_{p}$ & 0.01 & 0.02 & 0.02 & 0.02 & 0.02 \\
\hline$\theta$ & 0.14 & 0.18 & 0.12 & 0.15 & 0.15 \\
\hline$\sigma_{\text {in }}^{\text {tail }}$ & 4.9 & 4.4 & 3.3 & 3.2 & 3.5 \\
\hline$\sigma_{\mathrm{qe}}^{\text {tail }}$ & 1.1 & 0.87 & 0.68 & 0.50 & 0.57 \\
\hline $\begin{array}{c}\sigma_{\text {laca }}^{\text {tail }} \\
\text { tal }\end{array}$ & 0.41 & 0.24 & 0.30 & 0.31 & 0.45 \\
\hline$\sigma_{\text {tal. }}^{\text {tail }}$ & 9.3 & 7.9 & 6.4 & 7.1 & 9.3 \\
\hline$\sigma_{\mathrm{e}}^{\text {tail }}$ & 0.02 & 0.009 & 0.008 & 0.006 & 0.007 \\
\hline$\sigma_{\mathrm{le} \text { tail }}^{\text {tail }}$ & 0.02 & 0.008 & 0.010 & 0.01 & 0.015 \\
\hline$\sigma_{\text {t.e }}^{\text {tail }}$ & 0.17 & 0.10 & 0.10 & 0.11 & 0.18 \\
\hline$\sigma_{\text {t.in }}^{\text {tail }}$ & 6.9 & 0.8 & 0.7 & 1.8 & 2.4 \\
\hline$\sigma_{\text {l.in }}^{\text {tail }}$ & 0.07 & 0.11 & 0.12 & 0.32 & 0.41 \\
\hline$P_{n}$ & 8.8 & 6.0 & 5.1 & 5.2 & 4.7 \\
\hline$P_{p}$ & 0.00 & 0.00 & 0.00 & 0.00 & 0.00 \\
\hline$q_{1}^{p}$ & 0.00 & 0.00 & 0.00 & 0.00 & 0.00 \\
\hline$g_{2}^{p}$ & 8.8 & 4.8 & 3.0 & 3.2 & 1.8 \\
\hline TOTAL & 25.3 & 25.9 & 21.2 & 26.8 & 29.9 \\
\hline
\end{tabular}

Table 10.3: Systematic errors on $g_{2}^{n}$ by variable given in percent of $g_{2}^{n}$. 


\section{APPENDIX A}

\section{Magnetic Field Calibrations}

Fig. A.1 and Fig. A.2 are plots of the holding field calibrations as described in section 6.8.1.
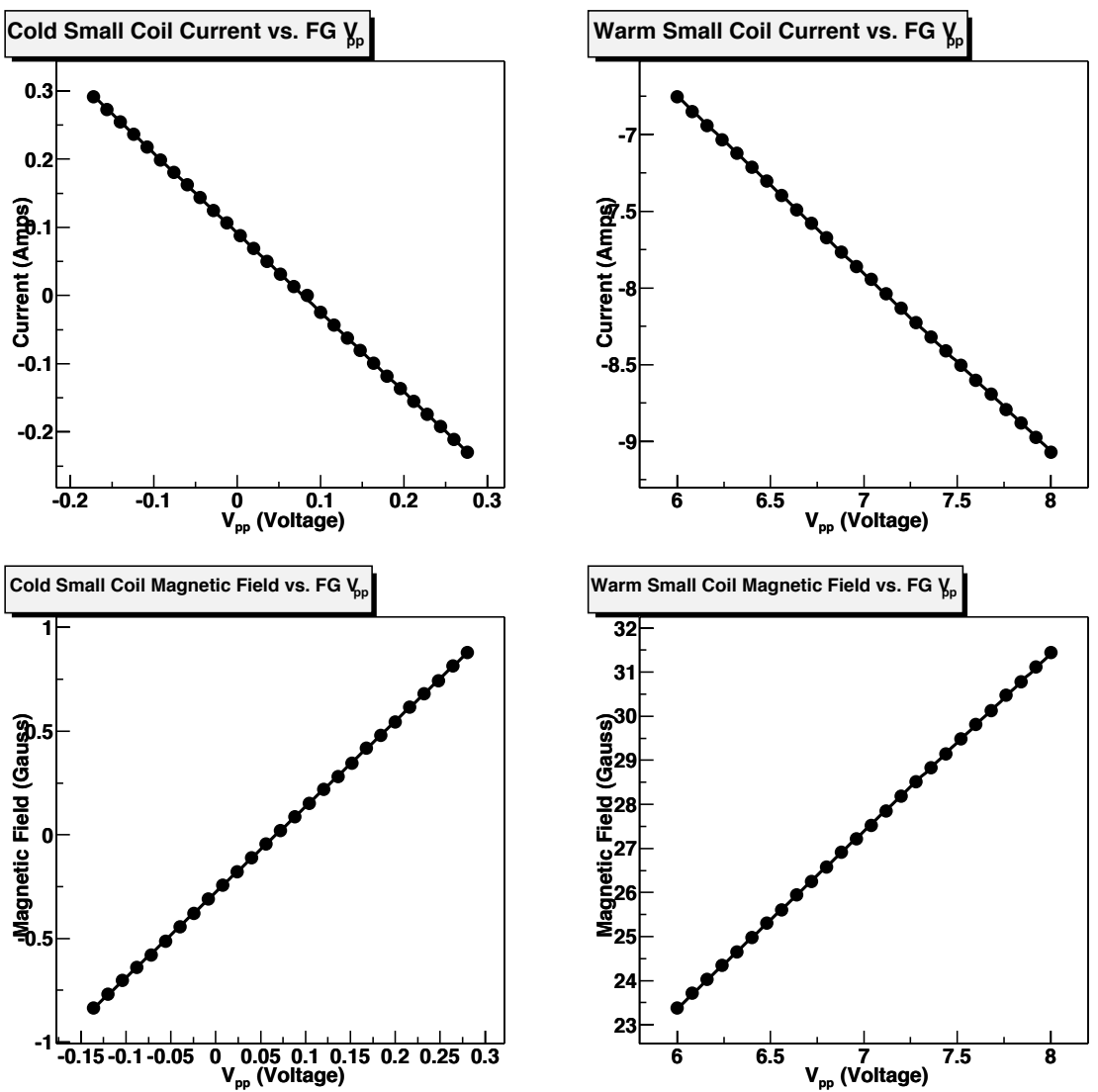

Figure A.1: The calibrations of the small Helmholtz coils. 

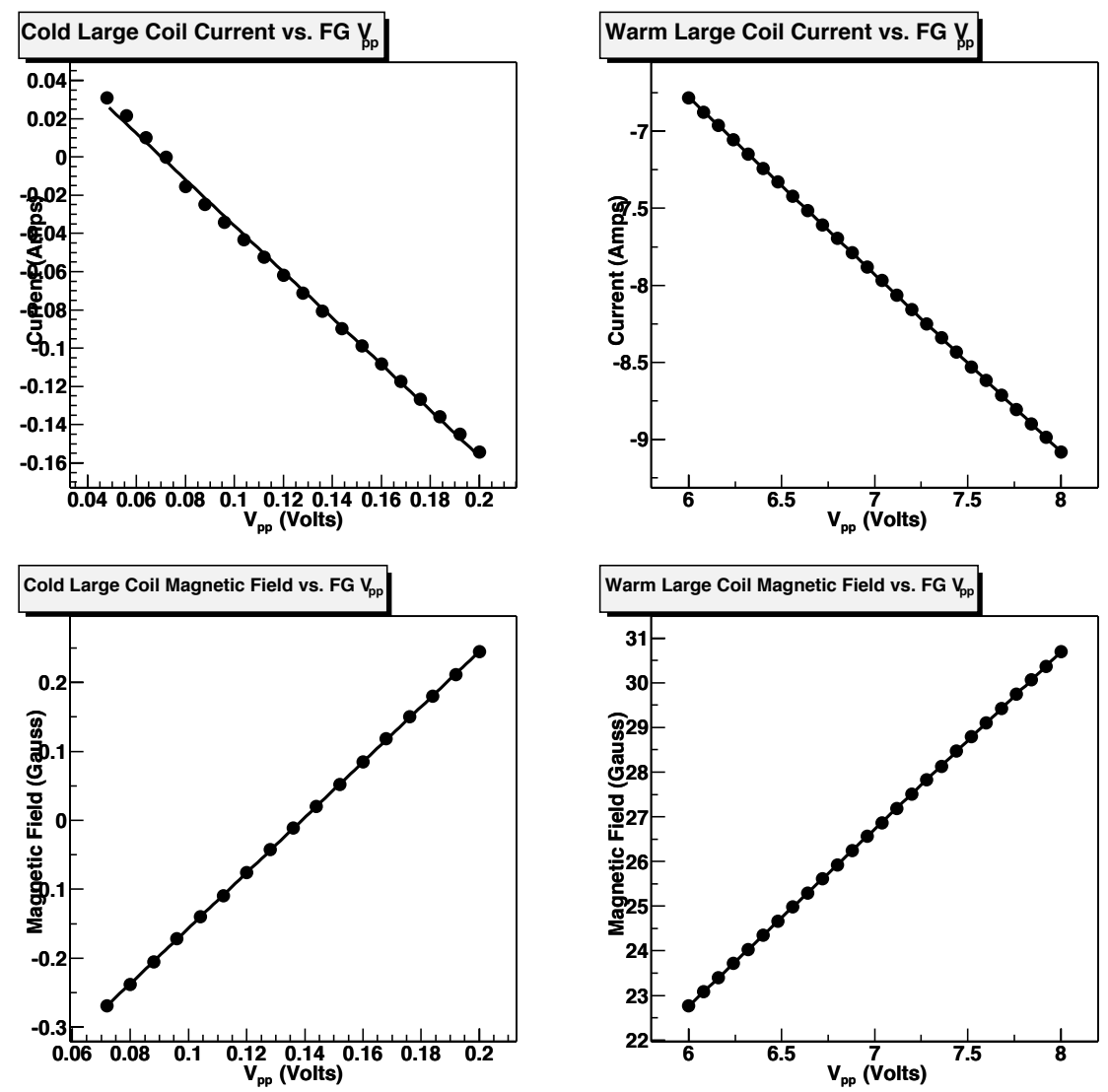

Figure A.2: The calibrations of the large Helmholtz coils. 


\section{APPENDIX B}

\section{Mapping of Holding Field Gradients}

Fig. B.1 and Fig. B.2 are results of measuring the gradients of the holding field in the small and large coils detailed in section 6.8.2.
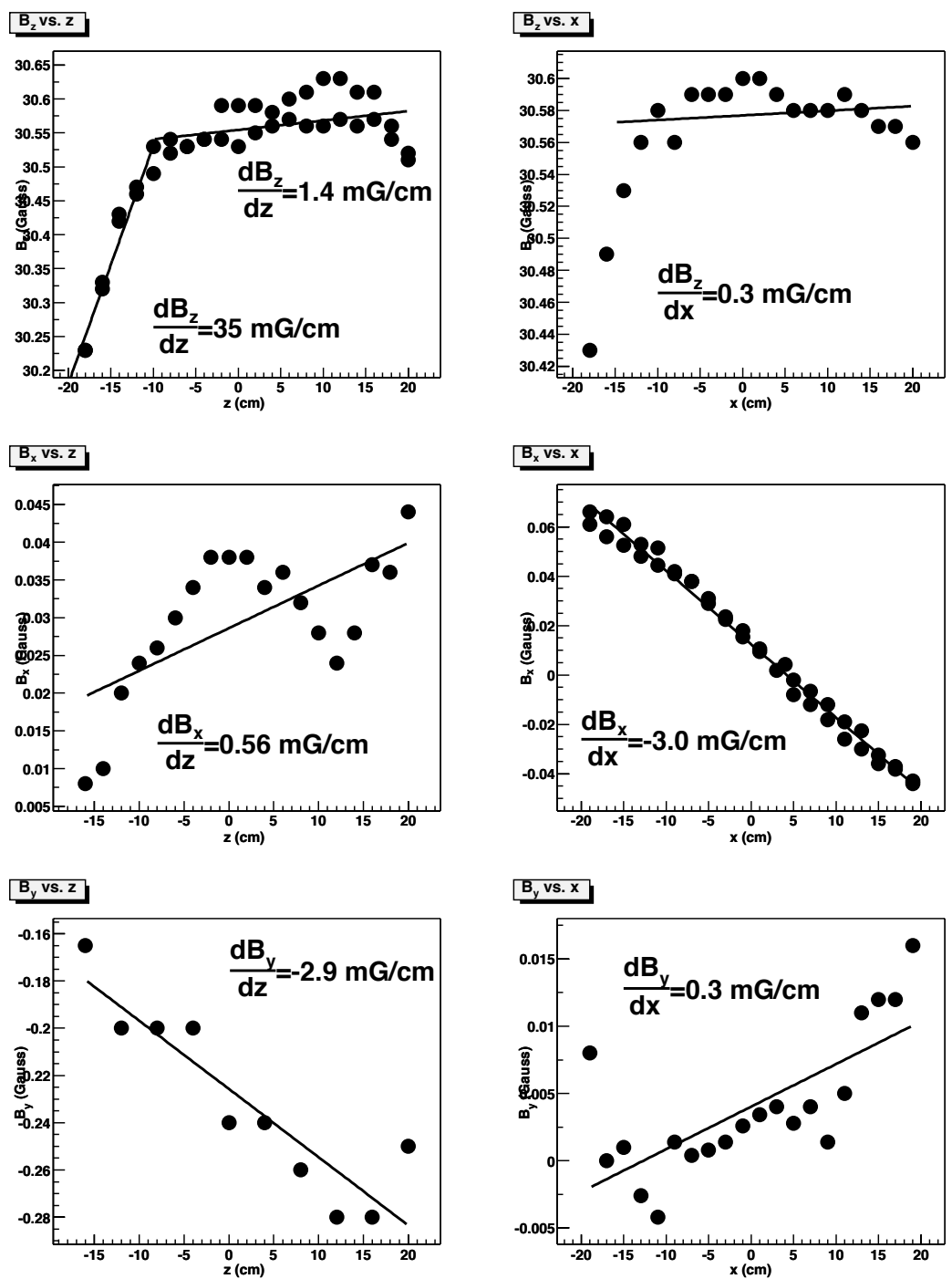

Figure B.1: Holding field gradient measurements of the small Helmholtz coils. 
B zs. z

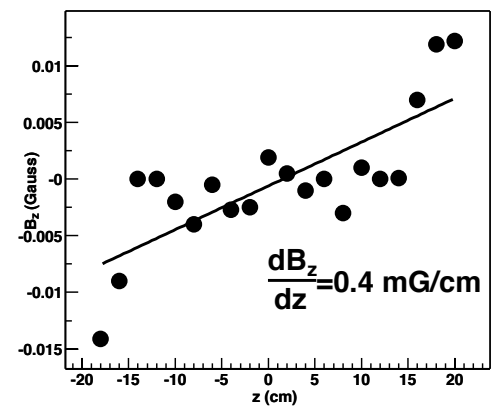

B. VS. z
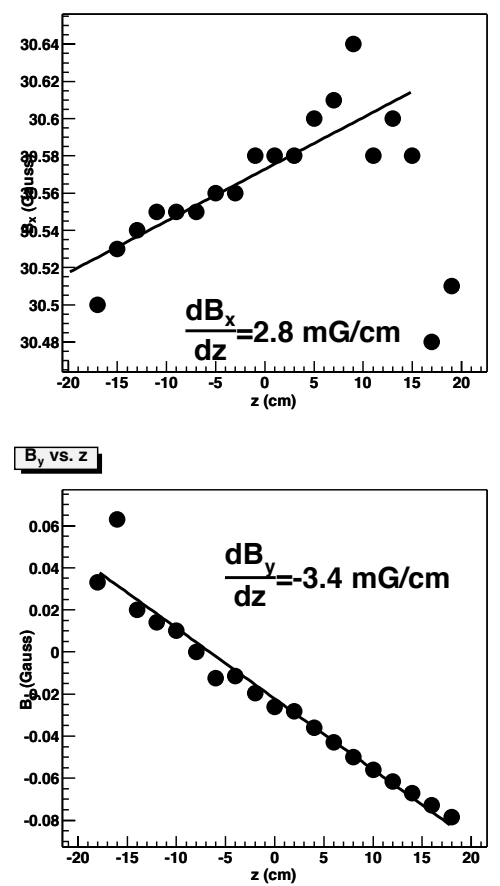

$B_{z}$ vs. X

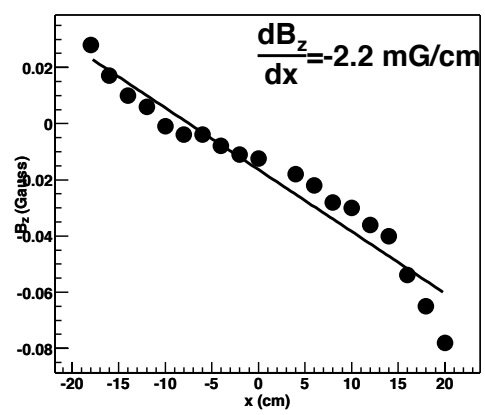

B. VS. $x$

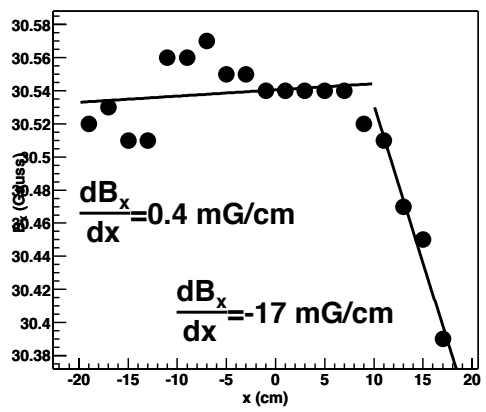

By vs. x

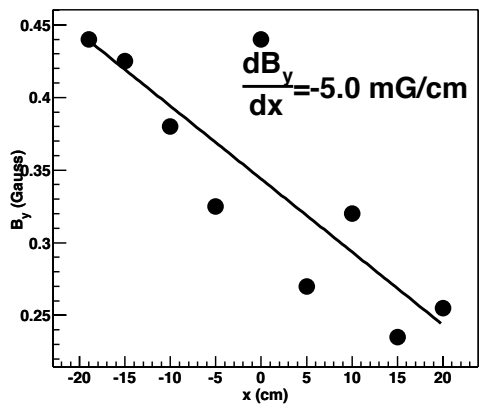

Figure B.2: Holding field gradient measurements for the large Helmholtz coils. 


\section{APPENDIX C Results of Field Direction Surveys}

There were four sets of surveys done of the polarized ${ }^{3} \mathrm{He}$ target holding field direction. All four were done with a compass and survey team as described in section 6.8.3. The surveyed angles are listed below accompanied by the calculations of the holding field direction based on the coil currents and the difference between the two. The currents in the gradient coils and some comments about the measurements are also listed.

\begin{tabular}{|ccc|l|}
\hline \hline & \multicolumn{3}{c|}{24 May 2001 } \\
\hline Compass $\theta$ & Calibration $\theta$ & $\Delta \theta$ & Comments \\
\hline-0.230 & -0.030 & -0.200 & flourescent light in scatt. chamber \\
-0.200 & -0.030 & -0.170 & flourescent light removed \\
180.260 & 179.971 & 0.289 & flip field direction \\
270.250 & 270.000 & 0.250 & set field transverse \\
269.940 & 269.798 & 0.142 & adjust field closer to $270^{\circ}$ \\
88.930 & 88.918 & 0.012 & flip field direction \\
89.770 & 89.775 & -0.005 & adjust field closer to $90^{\circ}$ \\
89.830 & 89.775 & 0.055 & set left arm Q1 to 3000 Amps \\
\hline \hline
\end{tabular}

Table C.1: Comparison of the results for the field direction of the compass survey with the holding field calibration. 


\begin{tabular}{|c|c|c|c|c|c|}
\hline \multicolumn{6}{|c|}{9 July 2001} \\
\hline $\begin{array}{c}\text { Compass } \\
\theta \\
\end{array}$ & $\begin{array}{c}\text { Calibration } \\
\theta \\
\end{array}$ & $\Delta \theta$ & $\begin{array}{l}\text { L. Grad. } \\
\text { I (Amps) }\end{array}$ & $\begin{array}{l}\text { T. Grad. } \\
\text { I (Amps) }\end{array}$ & Comments \\
\hline 0.040 & -0.033 & 0.073 & 0.0 & 0.0 & \\
\hline 180.430 & 179.962 & 0.468 & 0.0 & 7.0 & \\
\hline 180.450 & 179.965 & 0.485 & 6.0 & 0.0 & \\
\hline 180.440 & 179.965 & 0.475 & 6.0 & 0.0 & \\
\hline 180.460 & 179.965 & 0.495 & 5.0 & 2.0 & \\
\hline 180.430 & 179.965 & 0.465 & 0.0 & 0.0 & adjusting current to $180^{\circ}$ \\
\hline 180.270 & 179.982 & 0.288 & 0.0 & 0.0 & \\
\hline 180.310 & 180.003 & 0.307 & 0.0 & 0.0 & \\
\hline 180.310 & 180.023 & 0.286 & 0.0 & 0.0 & \\
\hline 180.330 & 180.136 & 0.194 & 0.0 & 0.0 & \\
\hline 179.580 & 177.908 & 1.672 & 0.0 & 0.0 & \\
\hline 179.650 & 179.021 & 0.629 & 0.0 & 0.0 & \\
\hline 180.030 & 179.578 & 0.452 & 0.0 & 0.0 & \\
\hline 270.250 & 269.998 & 0.252 & 0.0 & 0.0 & \\
\hline 270.260 & 269.998 & 0.262 & 5.0 & 2.0 & \\
\hline 270.260 & 269.998 & 0.262 & 7.0 & 0.0 & \\
\hline 270.250 & 269.998 & 0.252 & 0.0 & 7.0 & \\
\hline 90.250 & 90.119 & 0.131 & 0.0 & 7.0 & \\
\hline 90.260 & 90.120 & 0.140 & 6.0 & 0.0 & \\
\hline 90.250 & 90.120 & 0.130 & 5.0 & 2.0 & \\
\hline 90.260 & 90.121 & 0.139 & 0.0 & 0.0 & \\
\hline 270.180 & 269.998 & 0.182 & 0.0 & 0.0 & \\
\hline 270.570 & 269.998 & 0.572 & 0.0 & 0.0 & after tapping compass \\
\hline 0.300 & -0.029 & 0.329 & 0.0 & 0.0 & \\
\hline 0.230 & -0.029 & 0.259 & 0.0 & 0.0 & after tapping compass \\
\hline
\end{tabular}

Table C.2: Comparison of the results for the field direction of the compass survey with the holding field calibration. 


\begin{tabular}{|ccc|cc|l|}
\hline \hline \multicolumn{5}{c}{2 Aug 2001 } \\
\hline Compass & Calibration & $\Delta \theta$ & L. Grad. & T. Grad. & Comments \\
$\theta$ & $\theta$ & & I (Amps) & I $($ Amps $)$ & \\
\hline-0.060 & -0.026 & -0.034 & 0.0 & 0.0 & Spectrometer $45^{\circ}$ \\
-0.040 & -0.026 & -0.014 & 5.0 & 5.0 & \\
180.530 & 179.961 & 0.569 & 0.0 & 0.0 & \\
180.290 & 179.962 & 0.328 & 0.0 & 0.0 & \\
180.270 & 179.961 & 0.309 & 0.0 & 0.0 & after tapping compass \\
90.590 & 90.125 & 0.465 & 0.0 & 0.0 & \\
90.570 & 90.126 & 0.444 & 0.0 & 0.0 & after tapping compass \\
90.570 & 90.127 & 0.443 & 5.0 & 5.0 & \\
270.290 & 269.998 & 0.292 & 0.0 & 0.0 & \\
270.280 & 269.998 & 0.282 & 5.0 & 5.0 & \\
-0.450 & -0.022 & -0.428 & 0.0 & 0.0 & Spectrometer 18.6 \\
-0.290 & -0.023 & -0.267 & 0.0 & 0.0 & after tapping compass \\
-0.530 & -0.264 & -0.266 & 0.0 & 0.0 & changed current \\
0.020 & 0.218 & -0.198 & 0.0 & 0.0 & changed current \\
-0.060 & 0.219 & -0.279 & 5.0 & 5.0 & tapped compass \\
180.170 & 179.958 & 0.212 & 0.0 & 0.0 & \\
270.660 & 270.000 & 0.660 & 0.0 & 0.0 & \\
270.510 & 270.000 & 0.510 & 0.0 & 0.0 & \\
271.180 & 270.638 & 0.542 & 0.0 & 0.0 & changed current \\
270.020 & 269.438 & 0.582 & 0.0 & 0.0 & changed current \\
91.030 & 90.002 & 1.028 & 0.0 & 0.0 & \\
89.920 & 88.930 & 0.990 & 0.0 & 0.0 & changed current \\
89.910 & 88.929 & 0.981 & 5.0 & 5.0 & \\
89.490 & 88.929 & 0.561 & 0.0 & 0.0 & moved compass, Q1-R off \\
89.510 & 88.927 & 0.583 & 0.0 & 0.0 & \\
\hline \hline
\end{tabular}

Table C.3: Comparison of the results for the field direction of the compass survey with the holding field calibration. 


\begin{tabular}{|ccc|cc|l|}
\hline \hline \multicolumn{5}{c|}{18 Aug 2001 } \\
\hline \begin{tabular}{ccc|c} 
Compass \\
$\theta$
\end{tabular} & Calibration & $\Delta \theta$ & L. Grad. & T. Grad. & Comments \\
\hline 269.610 & 269.456 & 0.154 & 0.0 & 0.0 & \\
269.590 & 269.458 & 0.132 & 2.0 & 5.0 & \\
269.590 & 269.464 & 0.126 & 2.0 & 5.0 & \\
89.080 & 89.131 & -0.051 & 0.0 & 0.0 & \\
89.110 & 89.131 & -0.021 & 2.0 & 5.0 & \\
89.080 & 89.131 & -0.051 & 0.0 & 0.0 & moved compass \\
89.080 & 89.129 & -0.049 & 0.0 & 0.0 & moved compass \\
89.090 & 89.128 & -0.038 & 0.0 & 0.0 & rotated compass \\
89.090 & 89.127 & -0.037 & 0.0 & 0.0 & changed fiducials \\
-0.480 & -0.262 & -0.218 & 0.0 & 0.0 & \\
-0.490 & -0.262 & -0.228 & 2.0 & 5.0 & \\
180.150 & 179.959 & 0.191 & 0.0 & 0.0 & \\
180.160 & 179.959 & 0.201 & 2.0 & 5.0 & \\
\hline \hline
\end{tabular}

Table C.4: Comparison of the results for the field direction of the compass survey with the holding field calibration. 


\section{BIBLIOGRAPHY}

[1] I Estermann and O. Stern. Magnetic moment of the deuton. Phys. Rev., 45:761, 1934.

[2] R. Frisch and O. Stern. Magnetic deflection of hydrogen molecules and the magnetic moment of the proton. Zeits. f. Physik, 85:4, 1933.

[3] R. W. Mcallister and R. Hofstadter. Elastic scattering of 188-mev electrons from the proton and the alpha particle. Phys. Rev., 102:851-856, 1956.

[4] Richard P. Feynman. Very high-energy collisions of hadrons. Phys. Rev. Lett., 23:1415-1417, 1969.

[5] Murray Gell-Mann. A schematic model of baryons and mesons. Phys. Lett., 8:214-215, 1964.

[6] G. Zweig. An su(3) model for strong interaction symmetry and its breaking. 2. CERN-TH-412.

[7] A. Thomas and W. Weise. The Structure of the Nucleon. Wiley-VCH, 2001.

[8] S. Weinberg. A model of leptons. Phys. Rev. Lett., 17:616, 1967.

[9] N. Svartholm, editor. Elementary particle theory, relativistic groups and analyticity:proceedings. Wiley, 1968.

[10] S. L. Glashow, J. Iliopoulos, and L. Maiani. Weak interactions with lepton hadron symmetry. Phys. Rev., D2:1285-1292, 1970.

[11] J. D. Bjorken and Emmanuel A. Paschos. Inelastic electron proton and gamma proton scattering, and the structure of the nucleon. Phys. Rev., 185:1975-1982, 1969.

[12] Y. Kolomensky. Precision Measurement of the Neutron Spin Dependent Structure Functions. PhD thesis, University of Massachusets-Amherst, 1997. 
[13] B. Fillipone. Hugs seminar, 1999.

[14] O. Hansen. Hugs seminar, 1999.

[15] R.-W. Schulze and P. U. Sauer. Inelastic electron scattering from the three-nucleon bound states with polarization. Phys. Rev. C, 48(1):38, July 1993.

[16] A. Amroun et al. H-3 and he-3 electromagnetic form-factors. Nucl. Phys., A579:596-626, 1994.

[17] F. Bissey, V. Guzey, M. Strikman, and A. W. Thomas. Complete analysis of spin structure function g1 of he-3. Phys. Rev., C65:064317, 2002.

[18] J. W. Lightbody and J. S. O'Connell. Modeling single arm electron scattering and nucleon production from nuclei by gev electrons. Computers in Physics, page 57, May/June 1988.

[19] W. Leidemann et al. Asymmetry in inclusive polarized electron scattering from polarized nuclei:sum rule approach. Phys. Rev C, 42(1):416, July 1990.

[20] S. Choi. Private communication.

[21] The New Muon Collaboration. Measurement of the proton and deuteron structure functions $\mathrm{f} 2 \mathrm{p}$ and $\mathrm{f} 2 \mathrm{~d}$ and of the ratio sigmal/sigmat. Nuclear Physics B, 483:3, 1997.

[22] S. Wandzura and Frank Wilczek. Sum rules for spin dependent electroproduction: Test of relativistic constituent quarks. Phys. Lett., B72:195, 1977.

[23] M. Peskin and D. Schroeder. An Introduction to Quantum Field Theory. Addison-Wesley, 1997.

[24] Aneesh V. Manohar. An introduction to spin dependent deep inelastic scattering. eprint hep-ph/9204208, March 1992.

[25] B. W. Filippone and Xiang-Dong Ji. The spin structure of the nucleon. Adv. Nucl. Phys., 26:1, 2001.

[26] R. L. Jaffe and Xiang-Dong Ji. Studies of the transverse spin dependent structure function $\mathrm{g}(2)$ (x, q**2). Phys. Rev., D43:724-732, 1991. 
[27] Prakash Mathews, V. Ravindran, and K. Sridhar. Transverse structure function in the factorisation method, 1996. hep-ph/9607385.

[28] A. J. G. Hey and J. E. Mandula. Light cone analysis of spin dependent deep inelastic electron scattering. Phys. Rev., D5:2610-2614, 1972.

[29] X. Song and J. S. McCarthy. Model calculation of nucleon structure functions. Phys. Rev., D49:3169-3186, 1994.

[30] X. Song. Polarized structure function $g_{2}$ in the cm bag model. Phys. Rev., D54:1955-1966, 1996.

[31] M. Stratmann. Bag model predictions for polarized structure functions and their q**2 evolutions. Z. Phys., C60:763-772, 1993.

[32] K. Abe et al. Measurements of the proton and deuteron spin structure functions g1 and g2. Phys. Rev., D58:112003, 1998.

[33] A. Airapetian et al. Measurement of the proton spin structure function g1(p) with a pure hydrogen target. Phys. Lett., B442:484-492, 1998.

[34] P. L. Anthony et al. Measurements of the $\mathrm{q}^{* *} 2$ dependence of the proton and neutron spin structure functions g1(p) and g1(n). Phys. Lett., B493:19-28, 2000.

[35] B. Adeva et al. Spin asymmetries a(1) and structure functions g1 of the proton and the deuteron from polarized high energy muon scattering. Phys. Rev., D58:112001, 1998.

[36] J. Ashman et al. Measurement of the ratios of deep inelastic muon - nucleus cross-sections on various nuclei compared to deuterium. Phys. Lett., B202:603, 1988.

[37] K. Abe et al. Precision measurement of the deuteron spin structure function g1(d). Phys. Rev. Lett., 75:25-28, 1995.

[38] P. L. Anthony et al. Deep inelastic scattering of polarized electrons by polarized he-3 and the study of the neutron spin structure. Phys. Rev., D54:6620-6650, 1996.

[39] K. Ackerstaff et al. Measurement of the neutron spin structure function g1(n) with a polarized he-3 internal target. Phys. Lett., B404:383-389, 1997. 
[40] K. Abe et al. Precision determination of the neutron spin structure function g1(n). Phys. Rev. Lett., 79:26-30, 1997.

[41] X. Zheng. Precision Measurement of Neutron Spin Asymmetry $A_{1}^{n}$ at Large $x_{B j}$ Using CEBAF at $5.7 \mathrm{GeV}$. PhD thesis, Massachusetts Institute of Technology, December 2002.

[42] C. W. Leemann, D. R. Douglas, and G. A. Krafft. The continuous electron beam accelerator facility: Cebaf at the jefferson laboratory. Ann. Rev. Nucl. Part. Sci., 51:413-450, 2001.

[43] R. Alley et al. The stanford linear accelerator polarized electron source. Nucl. Instrum. Meth., A365:1-27, 1995.

[44] The Jefferson Lab Hall A Collabortaion. Basic instrumentation for hall a at jefferson lab. To be published, April 2003.

[45] J. P. Jorda et al. A fabry-perot cavity for compton polarimetry. Nucl. Instrum. Meth., A412:1-18, 1998.

[46] J. Grames. Unique electron polarimeter comparison and spin-based energy measurement. Presented at IEEE Particle Accelerator Conference (PAC2001), Chicago, Illinois, 18-22 Jun 2001.

[47] C. Jutier. Measurement of Virtual Compton Scattering Below Pion Threshold at Invariant Four-Momentum Transfer Squared $Q^{2}=1 .(\mathrm{GeV} / \mathrm{C})^{2} . \mathrm{PhD}$ thesis, Old Dominion University, 2001.

[48] J. et al Mitchel. Hall A Experimental Equipment Operations Manual. The Hall A Collaboration, February 2000.

[49] K. Hagiwara et al. Review of particle physics. Phys. Rev., D66:010001, 2002.

[50] P. L. Anthony et al. Precision measurement of the proton and deuteron spin structure functions g2 and asymmetries a(2). Phys. Lett., B553:18-24, 2003.

[51] T. D. Averett et al. A solid polarized target for high-luminosity experiments. Nucl. Instrum. Meth., A427:440-454, 1999.

[52] K. Abe et al. Measurement of the neutron spin structure function g2(n) and asymmetry a2(n). Phys. Lett., B404:377-382, 1997. 
[53] John Steffen Jensen. Measurement of the Neutron $\left({ }^{\beta} H e\right)$ Spin Structure Function at Low $Q^{2}$ : a connection between the Bjorken and Drell-Hearn-Gerasimov sum rules. PhD thesis, California Institute of Technology, 2000.

[54] C. E. Jones et al. He-3 (polarized) (e (polarized), e-prime) quasielastic asymmetry. Phys. Rev., C47:110-130, 1993.

[55] J. Becker et al. Determination of the neutron electric form factor from the reaction he-3(e,e' n) at medium momentum transfer. Eur. Phys. J., A6:329-344, 1999.

[56] T. et al Chupp. Polarized, high-density, gaseous ${ }^{3}$ He targets. Phys. Rev. C, $36: 2244,1987$.

[57] Mikhail V. Romalis. Laser - polarized He-3 target used for a precision measurement of the neutron spin structure. PhD thesis, Princeton Unversity, 1997. UMI-97-27062.

[58] N. et al Newbury. Gaseous ${ }^{3}$ He magnetic dipolar spin relaxation. Phys. Rev. $A, 48: 4411,1993$.

[59] K. et al Coulter. Measurement of ${ }^{3} \mathrm{He}$ depolarization rates during bombardment with a ${ }^{4}$ he beam. Nucl. Instr. Meth. A, 276:29, 1989.

[60] K.D. et al Bonin. Relaxation of gaseous spin-polarized ${ }^{3} \mathrm{He}$ targets due to ionizing radiation. Phys. Rev. A, 37(9):3270, 1988.

[61] Ioannis Kominis. Measurement of the Neutron $\beta$ He) Spin Structure at Low $Q^{2}$ and the Extended Gerasimov-Drell-Hearn Sum Rule. PhD thesis, Princeton University, 2001.

[62] M. Amarian et al. The $\mathrm{q}^{* * 2} 2$ evolution of the generalized gerasimov-drell-hearn integral for the neutron using a he-3 target. Phys. Rev. Lett., 89:242301, 2002.

[63] W. Xu et al. The transverse asymmetry a(t') from quasielastic polarized he-3(pol.)(e(pol.),e') process and the neutron magnetic form factor. Phys. Rev. Lett., 85:2900-2904, 2000.

[64] W. H. Shoup. Private Communication.

[65] E. Hecht. Optics. Addison-Wesley, 1998. 
[66] A. Deur. E94-010 tech. note \# 21 : Magnetic field cartography of the hall a ${ }^{3}$ he polarized target. Technical report, Jefferson Lab, 1998.

[67] S. Incerti. E94-010 tech. note \# 9: The nmr system of the hall a polarized ${ }^{3}$ he target. Technical report, Jefferson Lab, 1998.

[68] Jefferon Lab Survey Team. private communication.

[69] A. Abragam. Principles of Nuclear Magnetism. Oxford University Press, 1961.

[70] A. Thompson. Measurement of Asymmetries in Inclusive Quasi-Elastic Scattering of Polarized Electrons from Polarized ${ }^{3} \mathrm{He}$. PhD thesis, Massachusets Institute of Technology, 1991.

[71] S. Incerti. E94-010 tech. note \# 10 : Model of the water signal shape from the bloch equations. Technical report, Jefferson Lab, 1998.

[72] S. Incerti. E94-010 tech. note \# 22 : Monitoring of the pick-up coils. Technical report, Jefferson Lab, 1999.

[73] S. Incerti. E94-010 tech. note \# 15: Effect of the lock-in time constant on the nmr signal shape. Technical report, Jefferson Lab, 1998.

[74] W. et al Press. Numerical Recipes in C:The Art of Scientific Computing. Cambridge, 2nd edition, 1992.

[75] A. Deur. Etude Experimentale de la Structure en Spin du Neutron $\left({ }^{\beta} \mathrm{He}\right)$ a Bas $Q^{2}$ :Une Connexion Entre Les Regles de Somme de Bjorken et Gerasimov-Drell-Hearn. PhD thesis, Universite Blaise Pascal, 2000.

[76] N. Liyange. A study of the ${ }^{16} O(e, e, p)$ reaction at deep missing energies. $\mathrm{PhD}$ thesis, Massachusets Institute of Technology, 1999.

[77] K. Fissum. Hall A VDC Manual V2.1. Massachusets Institute of Technology, 1997.

[78] A. Deur. E94-010 tech. note \# 3 : Knock-on of $\delta$-electrons in hall a electron arm. effect of the aerogel cerenkov. Technical report, Jefferson Lab, 1998.

[79] The Hall A Collaboration. ESPACE Manual, 2.9.0 $\beta$ edition.

[80] B. Reitz. Private communication. 
[81] R. Gilman. Private communication.

[82] D. Armstrong. Private communication.

[83] A. Deur. Single arm monte carlo for polarized 3he experiments in hall a v.0.2. Hall A Tech Note JLab-TN-01-004, Jefferson Lab, 2001.

[84] I. Akushevich, A. Ilichev, N. Shumeiko, A. Soroko, and A. Tolkachev. Polrad 2.0: Fortran code for the radiative corrections calculation to deep inelastic scattering of polarized particles. Comput. Phys. Commun., 104:201-244, 1997.

[85] T. V. Kukhto and N. M. Shumeiko. Electromagnetic effects in deep inelastic scattering of polarized leptons on polarized nucleons. (in russian). Yad. Fiz., 36:707-720, 1982.

[86] I. V. Akushevich and N. M. Shumeiko. Radiative effects in deep inelastic scattering of polarized leptons by polarized light nuclei. J. Phys., G20:513-530, 1994.

[87] Luke W. Mo and Yung-Su Tsai. Radiative corrections to elastic and inelastic e p and mu p scattering. Rev. Mod. Phys., 41:205-235, 1969.

[88] S. Stein et al. Electron scattering at 4-degrees with energies of 4.5-gev 20-gev. Phys. Rev., D12:1884, 1975.

[89] X. Zheng. Private communication.

[90] J. S. O'Connell et al. Electromagnetic excitation of the delta resonance in nuclei. Phys. Rev., C35:1063, 1987.

[91] K. Slifer. E94-010 tech. note \# 44 : Modifications to the qfs and radcor codes. Technical report, Jefferson Lab, 2003.

[92] J. P. Chen. Private communication.

[93] M. Arneodo et al. Measurement of the proton and the deuteron structure functions, f2(p) and f2(d). Phys. Lett., B364:107-115, 1995.

[94] Y. Liang. Private communication.

[95] W. Korsch. Private communication.

[96] J. Blumlein and H. Bottcher. Qcd analysis of polarized deep inelastic scattering data and parton distributions. Nucl. Phys., B636:225-263, 2002. 


\title{
VITA
}

\author{
$\underline{\text { Kevin Michael Kramer }}$
}

Kevin Michael Kramer was born in Rockledge, Florida, on 30 October 1972. Graduated from Langham Creek High School in Houston, Texas, in May 1991. Received a Bachelor of Science degree in physics from the University of Texas at Austin in Austin, Texas in May 1995. Entered the physics graduate program at the College of William and Mary in Virginia in 1997. Received a Master of Science degree in physics from the College of William and Mary in Virginia in May 1999. This dissertation was defended on some day in the future at the College of William and Mary in Virginia. 